\title{
Is the Body \\ the Temple of the Soul? \\ Modern Yoga Practice \\ as a Psychosocial Phenomenon
}


盗 


\author{
Krzysztof T. Konecki \\ Is the Body \\ the Temple of the Soul? \\ Modern Yoga Practice \\ as a Psychosocial Phenomenon
}


Krzysztof T. Konecki - University of Łódź, Faculty of Economics and Sociology Institute of Sociology, Department of Sociology of Organization \& Management 41/43 Rewolucji 1905 r., 90-214 Łódź

(C) Copyright by Krzysztof T. Konecki, Łódź 2015

(C) Copyright for this edition by University of Łódź, Łódź 2015

(c) Copyright for this edition by Jagiellonian University Press, Kraków 2015

All rights reserved

No part of this book may be reprinted or utilised in any form or by any electronic, mechanical or other means, now known or hereafter invented, including photocopying and recording, or in any information storage or retrieval system, without permission in writing from the publishers

Published by Łódź University Press \& Jagiellonian University Press

First edition, Łódź-Kraków 2015

ISBN 978-83-7969-819-6 - paperback Łódź University Press

ISBN 978-83-233-4008-9 - paperback Jagiellonian University Press

ISBN 978-83-7969-820-2 - electronic version Łódź University Press

ISBN 978-83-233-9282-8 - electronic version Jagiellonian University Press

Łódź University Press

8 Lindleya St., 90-131 Łódź

www.wydawnictwo.uni.lodz.pl

e-mail: ksiegarnia@uni.lodz.pl

phone +48 (42) 6655863

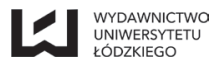

Distribution outside Poland

Jagiellonian University Press

9/2 Michałowskiego St., 31-126 Kraków

phone +48 (12) 6310197, +48 (12) 66323 81, fax +48 (12) 6632383

cell phone: +48 506006 674, e-mail: sprzedaz@wuj.pl

Bank: PEKAO SA, IBAN PL 80124047221111000048563325

www.wuj.pl

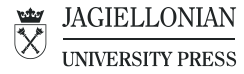




\section{Contents}

Foreword (Michał Szczepanik) . . . . . . . . . . . . . . . . 7

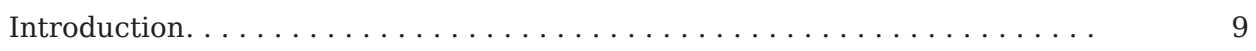

Chapter 1. Philosophy and hatha-yoga practice. Para-religious aspects

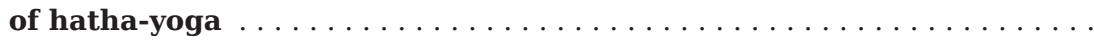

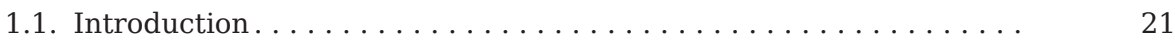

1.2. Yoga - the perspectives of religious studies scholar and the perspec-

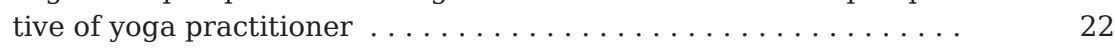

1.3. Yoga and society - "religion of everyday life" . . . . . . . . . . . 29

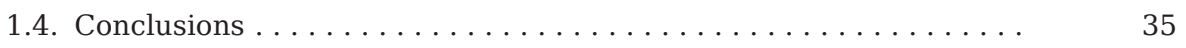

Chapter 2. The social world of yoga practice . . . . . . . . 39

2.1. What is the social world?. . . . . . . . . . . . . 39

2.2. Hatha-yoga practitioners and their social world - arenas and legiti-

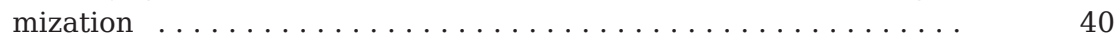

2.3. The intersection of the worlds. . . . . . . . . . 48

2.3.1. Intersection of the world of yoga practice and the world of

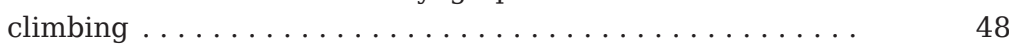

2.3.2. Intersection of the world of conventional medicine and alter-

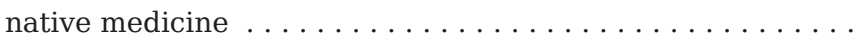

2.3.3. Intersection of the world of business corporations and the world of yoga practice . . . . . . . . . . . 50

2.3.4. Intersection of the world of yoga and pop-culture . . . . . 53

2.4. A disagreement about hatha-yoga origin. The vision of Marc Sin-

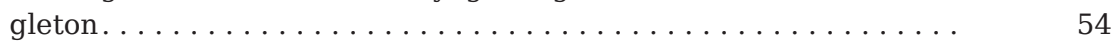

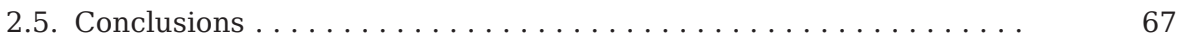

Chapter 3. Commonsense definitions of yoga and its meaning for prac-

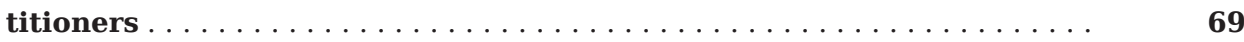

3.1. Introduction. . . . . . . . . . . . . . . . . . 69

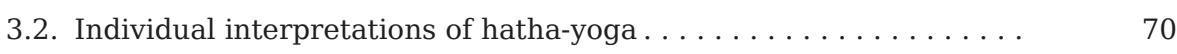

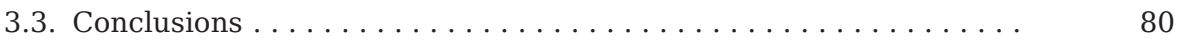

Chapter 4. The process of becoming a hatha-yoga practitioner. . . . . 83

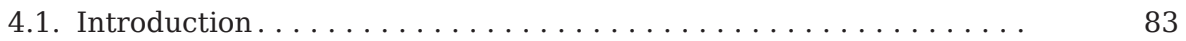

4.2. Introductory phase - construction of motives and first steps . . . . 85 


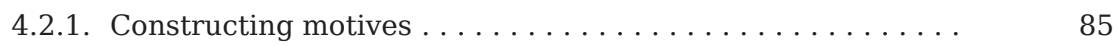

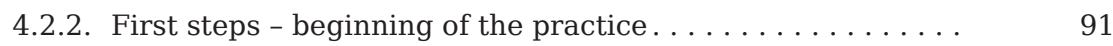

4.2.3. Noticing the effects . . . . . . . . . . . . . . 98

4.3. The phase of fuller recognition of psychophysical effects, and ascribing appropriate meanings to them . . . . . . . . . 105

4.4. Phase of fuller recognition of the spiritual (para-religious) aspects of

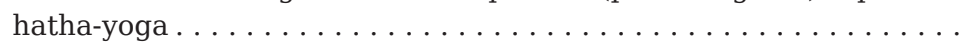

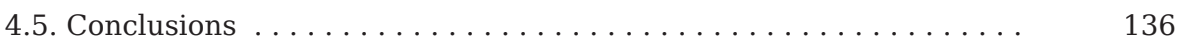

Chapter 5. Visual transmission of knowledge and the meaning of corporality and gestures in the social world of yoga practice . . . . . .

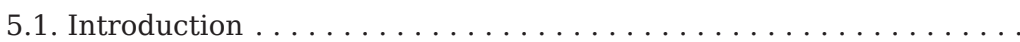

5.2. The method of studying corporality with the usage of visual tech-

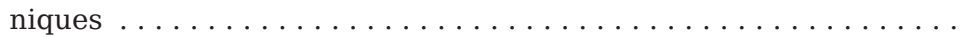

5.3. What do I see and feel when I practice? Analysis of the interviews

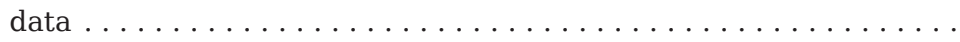

5.3.1. What do I see when somebody else practices? The analysis of films from the Internet used for interviews . . . . . . . . . . .

5.3.2. What do I feel when I practice? Analysis of the movie-elicited interviews . . . . . . . . . . . . . . . . . . .

5.3.3. Non-passable knowledge. Difficulties with verbal interpretations and description of asanas . . . . . . . . . .

5.3.4. Body empathy based on visualization. . . . . . . . . . .

5.4. The role of the teacher and the correctness of asana performance. .

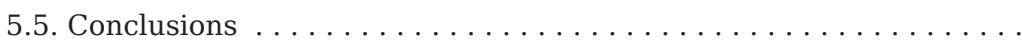

Chapter 6. Emotions and yoga practicing. Working on emotions and achieving "emotional culture" without emotions . . . . . . . . . . . .

6.1. Introduction . . . . . . . . . . . . . . . . . .

6.2. Working on emotions - explanations based on practitioners' statements............................. 183

6.3. Conclusions .......................... 190

Chapter 7. Teacher and guru in hatha-yoga practice . . . . . . . . . . 195

7.1. Introduction. . . . . . . . . . . . . . . . . . 195

7.2. Teacher-student interactions. . . . . . . . . . . . . . . . . 197

7.3. Conclusions . . . . . . . . . . . . . . . . . . . . . 207

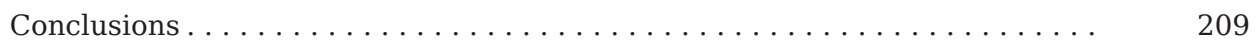

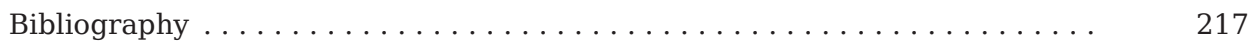




\section{Foreword}

Yoga is growing in popularity in Poland. As with every fashion, this leads to a number of simplifications, and sometimes even misunderstandings.

Meanwhile, the book by Professor Konecki is a book about yoga in the context of modern culture. It is also a book about modern culture in the context of yoga. It gathers the experience of trainees, and shows as well the phenomenon that evolved out of Indian tradition and became an integral part of Western-European lifestyle.

One may ask: in a world of corporations, business transactions, sports competitions and a lay world which is mistrustful towards everything that does not withstand the trial of rational discussion, is there space for the search for inner peace and harmony? How much does physicality (asana exercises) bind with spirituality? It is worth asking yourself these questions even if you have never set nor intend to set foot on the yoga mat. Maybe it is especially worth asking in a country like Poland, which is so dynamically changing and where the meaning of individual choices is becoming ever more important. Every thought which helps one harmonize the micro-cosmos that every human-being is, as well as consciously influence the macro-cosmos - the way the society functions and the way to treat the world we live in - is very valuable. Yoga, which may be treated as "the everyday life religion" (as described by Professor Konecki), or as a way to keep one's psycho-physical fitness in good shape, or as a chance to build community relationships, or connected with Indian culture, or maybe with the New Age, definitely offers one a chance to take control of his or her life. Even if we start from something as basic as our body, if we fully realize the influence that we have on it, that our body really belongs to us, maybe it will be easier to accommodate other aspects of our lives: conscious decision-making, human relations, and our use of natural resources and of the skills given to us. Is this a utopia? Perhaps, but perhaps too it is an opportunity that we overlook because it gets crushed by life's everyday pressures, and because we run away from difficult confrontations with here-and-now solutions.

The very title of Professor Konecki's book - Is the Body the Temple of the Soul? - creates a question from Iyengar's statement. At the same time, he makes the statement problematic by showing that yoga can 
also be treated as a display of Western-Europeans' detailed focus on fitness, or as a para-religion. The practice of yoga practice is always within the sphere of individual, private choices. This makes the usage of the tool, which asana exercise is, both easy and difficult. In the multitude of different and often equal propositions, the sole question about the sense, the essence of our experience or actions sometimes sounds naïve, even false. This can wash away the boundaries between yoga and regular visits to the gym, which can make the obtainment of pleasure from a yoga which does not comprise the physicality difficult. At the same time, spirituality is often dwarfed and exotic displays of it are treated suspiciously. But maybe the possibility of choice, of going down the path of yoga as the result of a personal search, allows for experimentation, for breaking through the externalities and searching for what is important for a given person.

Maybe too it is possible to look at this situation from a different angle. One of the aims of practicing yoga is to sharpen our attention, to build up the skill of penetration, without any prejudice or upfront judgments, into our perception of the world.

Does not the academic sociology have similar aims? In both cases we sit down in peace and quiet to observe what is. We do not try to change or improve anything at once. We concentrate on disciplining and making order of our busy everyday perceptions.

I myself have been practicing yoga for many years now, and it has resulted in very in-depth changes within me. My journeys to India, and acquaintance with the mystical traditions of the country that I have come across, have had a great influence on the kind of man I am today. Hence the scientific approach introduced in the work Is the Body the Temple of the Soul? interested me greatly. My reading of this book offered me the possibility to look from a distance at what I have created in my current life. As a teacher, I am happy to find that yoga has become a subject of such a thorough reflection. This book provides a way to show asana and meditation not only as a way of spending free time, but also as an important cultural factor.

Michał Szczepanik Hatha-Yoga Center, Łódź May 6, 2012 


\section{Introduction}

In modern Eastern societies, we are looking for something that would take us away from everyday life, from the workload and the problems connected with work, or the lack thereof. Everyday life is what aches us the most, but we have to go through it to achieve maturity and ask ourselves serious questions. This maturity is often connected with the will to understand oneself, the mind, and the body. The inherent limitations of these aspects are strongly visible when we cannot manage with the race of our thoughts connected with a purposeful, rational usage of time, which the harder we try to achieve, the more control we lose. Our mind often does not cope with the everyday routine that thickens more and more around us, where in our perception time often loses its linear character, being just a way to get us to a moment, in the form of meetings and making further plans and arrangements. Hence step by step we become weaker, both mentally and physically.

Feeling this, we often end up asking ourselves the question: "How can we stop this course?" Others may ask: "How can we improve the shape of their bodies and minds in order to manage with the slowly-consuming everyday life?" These two adult questions ("adult" not being connected here with biological age, but with one's state of mind) create a motivational base for the search to find a permanent and stable drift in everyday life, or to strengthen one's psycho-physical state in order to manage life. Thus begins an individual search. Some turn towards actions connected with other people and in this way realize that time is a relative notion, especially when we want to help others. We find time for them. Others find themselves in different types of hobbies, while some run away from everyday life by the simple act of travelling, and thus find themselves in the claws of the tourist agency world. Sport and physical activity also offer a possibility to improve one's psycho-physical state, as well as to escape from everyday life. Training and/or participation in marathons enables individuals to partially run away from everyday life, but still be strongly submerged, and boosting self-esteem is evident in a variety of different, everyday life aims, often not connected with sport. The ego is also working hard in sport.

In our modern Western societies, the industry of making our everyday life more attractive (or running away from it) is growing very 
rapidly. Tourist agencies, companies organizing physical culture, and entertainment industries are all trying to penetrate our everyday life, trying to obtain a tidbit of our free time in order to fill it up with something that will seem to make our everyday life less boring, to combat the state of suspension that arises from not doing anything, because not doing anything is poorly perceived in modern society, even widely condemned as a sin in everyday reality. Apart from businesses, different organizations, as well as individual units, offer us new ways of thinking, new forms of activity (often exotic and deriving from other cultural areas), far from the everyday routine constructed by ourselves on the basis of inherited values and norms, in an attempt to fill our free time.

In the context of this trend, we can include companies, institutes and organizations teaching us physical culture and training our bodies and minds, providing us with something to do. Training the body cannot take place without the mind. While training my body, I am also training my mind, and when I force myself to exercise, I shape endurance. By continually training for a longer period of time, I acquire new habits, creating my everyday routine from the start. I change my attitude towards my own body. I begin to perceive it as an interaction partner, which sometimes tries to resist me and other times transforms according to my will. I start perceiving the world through the prism of a body that reacts to outer stimuli.

Before, training the body was only a tool for the mind, a means to achieve an end. When it is the interaction partner, it becomes an active participant in the thinking processes, weighing the alternatives, and making decisions about the action(s) being taken. The body gives us signals that we are finally perceiving. Will I manage? The mind can fight the resistance of the body if it is our slave, a passive tool. However, it is so only up to a certain point. Physical exercises allow us to change the body into an active subject of action, combining with the mind in either managing everyday life, or running from it.

Hatha-yoga constitutes a practice of transforming body and mind so as at the end of this route there is a combining of these two entities, which are so often apart and pitted one against the other. Hatha-yoga in Western culture is often perceived as the practice of physical exercises (practice of the asana position), usually done to improve one's health and emotional state. Even if that is the case, it is still based on the rule of "the silencing of the modifications of the mind." It is about a containment of mind dispersion in terms of non-important, minute, everyday issues, which are not important for the basis of our existence, balance and inner harmony, the unity of body and mind. Even if we 
train only for our health and for improvement of our physical fitness, then the end result, in the case of many physical and sports activities, is stopping mind movement. The full concentration on one action, on one point in space, might not have any metaphysical meaning for us, but it still remains full concentration. This can be achieved by patiently practicing hatha-yoga.

The present monograph is an introduction to and a description of the world of social practices of hatha-yoga, the rules that govern it, motivations of those who practice it, and their definitions of situations in which they find themselves. This world is ever more popular and more and more people are practicing hatha-yoga. A whole new industry is growing based on hatha-yoga, which is visible even in our busy everyday lives. It is not without controversies. Some perceive the world of hatha-yoga as a sect and a danger for the mental health of those who practice it, as well as a danger for conventional religions. There are disputes about the source from which hatha-yoga originated; is it typically an Eastern practice or have esoteric Western exercises influenced its modern shape? Other disputes concern whether hatha-yoga is only a set of physical exercises or maybe a more metaphysical pursuit?

Yoga is becoming a more and more popular form of exercise. The research of the fitness market in 2010 shows that yoga occupies, among fitness clubs in Poland, the fourth position in their offer: 1) strengthening classes, aerobics - 100\%; 2) strength training - 98\%; 3) personal training - 98\%; 4) yoga - 88\% (Nelke 2010). Yoga is somewhat inscribed in the context of fitness. In Great Britain, it has the support of the government. Yoga was taught in the 1970s in the College of Physical Education, part of the Inner London Education Authority (Hasselle-Newcombe 2005: 305). Women are more likely to practice yoga than men (see: Górski 2004).

How did the social world of yoga develop in Poland? The reception of yoga in our country started a relatively long time ago. Worthy of mention are the crucial pioneering actions of Wincenty Lutosławski, who encountered the tradition of yoga while taking part in Vivekananda's lectures in 1893 in the U.S.A. (see: Lutosławski 1909). According to him, yoga was supposed to be useful in the healthy upbringing of youth in the spirit of patriotism. Lutosławski's interest in yoga was inspired by his health problems; thanks to yoga he cured himself of depression. ${ }^{1}$ Next, he familiarized himself with the works of Yoga Ramacharaka, and he both practiced the master's teaching himself and popularized it in Poland, where Ramacharaka's works were also pub-

\footnotetext{
${ }^{1}$ See: http://gabinetsurya.eu/?id=art-20120504-1803 (accessed: 30.12.2012).
} 
lished (Ramacharaka 1922; 1923; 1930). The beginnings of the medicalization of yoga seem to also be rooted in Lutosławski's actions those times.

Both the physical and medical aspects of yoga are also visible the in post-war years, in publications from the 1960s and 1970s (Ghose 1962; Bragdon 1970; Michalska 1974; Kogler 1975).

Hence therapeutic usages of yoga existed in our country from the beginning of its popularization. Wanda Dynowska and Maurycy Frydman greatly influenced the development of yoga in Poland. They created a Polish-Indian Library, where they popularized the idea of yoga for Poles and Polish culture in India. The teacher of Maurycy Frydman was initially Raman Maharishi, then Jiddu Krishnamurti. Frydman also wrote in English, bringing the Hindi culture and yoga itself closer to the Western culture. Wanda Dynowska had the same masters. She visited Poland twice, lecturing on Mahatma Gandhi, the philosophy of Krishnamurti, and Sri Raman Maharishi. She also translated books about yoga into Polish, as well as classical Indian religious books (Ghose 1962; Bragdon 1970).

Another propagator of yoga was Leon Cyboran. In 1973, he published his Ph.D. thesis: The Philosophy of Yoga - An Attempt at a New Interpretation, and in 1986 his Classical Indian Yoga of Yogasutra Ascribed to Patanjali and Yogabhashya, in Other Words a Comment to Yogasutra Ascribed to Vjasa (Cyboran 1973; 1986).

In the 1970s yoga was also popularized in TKKF (Towarzystwo Krzewienia Kultury Fizycznej [The Association of Popularizing Physical Culture]), which directed it towards physical and medical interpretations. A well-known person who popularized yoga was Tadeusz Pasek, a graduate of Poznan University of Economics, Bihar School of Yoga in Munger, a doctoral student of the Józef Piłsudski University of Physical Education in Warsaw, and an employee of the Psychiatric Clinic of Poznan University of Medical Sciences and the University of Toronto, Canada. ${ }^{2}$ He backed scientific research connected with analysis of the medical effectiveness of yoga. Malina Michalska, who was Lutosławski's student, also popularized yoga in the 1960s. She ran hatha-yoga classes on behalf of the Bihar School of Yoga, and also wrote a book popularizing yoga, Hatha-Yoga for Everyone (1974). Her book was also a form of the medicalization of yoga, as it was used mainly for healing purposes.

The medicalization of yoga in publications also continued after 1989, i.e. in the 1990s and continues today (Nagarathna, Monro 1990;

${ }^{2}$ Ibidem. 
Pūrnā 1996; Kulmatycki 1993; Górna, Szopa 2005; Garbara, Szopa 2013). Sometimes yoga is juxtaposed with other therapeutic techniques such as shiatsu, acupuncture, or visualization (Pilkington 1995). Yoga has also sometimes been psychologically oriented and supposed to help solve life problems (Niedźwiedź 1997).

According to an interview with Sławomir Bubicz, in the 1960s and 1970s there were fewer books published and they were connected mainly to the scientific interpretations concerning the physical and psychological sides of yoga (Moćko 2007). It also needs to be underlined that before the 1989 there were both publications accenting the health elements of yoga as well as scientific reports connected with hatha-yoga and other types of yoga that were distributed. Works by M. Eliade (1997) and L. Cyboran $(1973 ; 1986)$ were published, as well as popular science (Szyszko-Bohusz 1978). However, the physical and medical traditions of yoga were very strongly emphasized in many publications, reflecting the strong tendency at that time towards the medicalization of yoga in Poland.

In the 1980s a great propagator of hatha-yoga in Poland was Sławomir Bubicz, who then began publishing books. He later published some of them in a second edition, inasmuch as the censors of the 1980s did not like the mention of God in texts about yoga. The works in the 1980s also included Gabriella Gubillaro's books (Hatha-Yoga 1985, official publication with the intervention of censorship) and an extract of the book Light on Yoga by B. K. S. Iyengar, published in a second edition. In an interview, Sławomir Bubicz underscored the difficulties with the publication of books about yoga before the year $1989 .{ }^{3}$ One of the translations of Iyengar's books by Bubicz was released in 1999 (Yoga, Warsaw: PWN). In the $21^{\text {st }}$ century, Bubicz has been the publisher of many books by B. K. S. Iyengar and the greatest propagator of his version of hatha-yoga in Poland. Bubicz was in India for many years and learned directly from his master Iyengar.

As has been mentioned, in the 1990s academic positions about yoga, analyzing the academic traditions of Pantanjali, were also published (Jakubczak 1999). In addition, works medicalizing yoga as a way to help overcome stress, lose weight, maintain hormonal stability, or improve sight also appeared (Kulmatycki 1999), and this trend still continues in the $21^{\text {st }}$ century (Kulmatycki 2000; Teasdill 2001; Lichy 2004; Weintraub 2005; Maddern 2005). It also needs to be emphasized that there is a tendency to direct special offers of yoga exercises to women (Martínez 2009; Jaros 2011).

${ }^{3}$ See: http://www.youtube.com/watch?v=rTUWonM9RhY (accessed: 30.04.2012). 
Until now (2012), there have about 80 publications on yoga in Poland, including the pre-war ones. In the library of Warsaw University there are currently 74 positions in the Polish language that have the word "yoga" in the title. It needs to be underlined that this collection does not include all of the positions; in particular modern ones published by small, niche publishing houses are not included (e.g., Virya).

In Poland, the tradition of certifying yoga teachers has existed since the 1970s. Such certifications are given by the Association for Propagating Physical Culture and the Polish Association of Iyengar's Yoga. Specialists emphasize the danger of practicing yoga under the eye of unqualified instructors. This is especially underlined by practitioners who are connected with medicine and conventional physiotherapy (aforementioned). In many other schools the qualifications of yoga teachers are not checked, nor is inquiry made into where and by whom they were taught (Kulmatycki, Burzyński 2008: 166-167).

In Poland, post-graduate studies at the Academy of Physical Education in Wroclaw were opened, entitled "Post-graduate Studies of Psycho-somatic Yoga Practices." The research by Professor Lesław Kulmatycki and Zdzisław Burzyński shows a growing popularity of yoga schools in major cities. In addition, there is a Polish Association of Iyengar's Yoga (PSJI), whose aim is to maintain contact with the original Ramamani Iyengar Memorial Yoga Institute (RIMYI) in Pune, India, as well as to promote the training and development of yoga in Poland and grant certification to run hatha-yoga classes (Kulmatycki, Burzyński 2008: 160). In Poland, yoga instructors often refer to Iyengar's traditions. These traditions are referred to by teachers in 42 out of 56 schools analyzed by the researchers (ibid.: 169).

All of the above demonstrates that the institutionalization and legitimization of the social world of yoga practice is developing in Poland in a spontaneous fashion and is still not controlled in a formal and legal way. Hence various initiatives, coming from institutions widely recognized as universities or registered associations, are aimed at solving this problem by the certification of qualifications to run classes and yoga schools.

The information published in newspapers and magazines about the healthy effects of yoga also bears witness to the popularity of yoga exercises. Their positive effects on the psyche and the ability to concentrate are often underlined (Rokita 2008a; 2008b). Sometimes there are even supplements to magazines with specific exercises for specific pains. ${ }^{4}$ Various magazines often publish academic information about

\footnotetext{
${ }^{4}$ See: supplement to Shape 9 (126) in 2010, plus DVD Yoga for Back Rescue (in Polish).
} 
the medical effects of yoga practices, contributing to their popularity and social legitimization. This information may be, for example, shown in a particular form, such as: yoga has a calming effect because during exercises a gamma-aminobutyric acid is produced, which is a neurotransmitter calming down excited neurons. Journalists who are writing positively about yoga and showing famous people doing yoga, which helps increase its popularity (Staszewski 2009). There are also articles in the popular press referring to academic authorities that affirm the benevolent influences of yoga on our health (yoga lessens sleeplessness, reduces hypertension, improves insulin administration, and improves breathing, so it can help asthmatics) (Augustyn 2010). One may also find information about public yoga performances, such as a performance by hundreds of supporters in Times Square, New York, including photographs of such events. ${ }^{5}$ There are also articles presenting the results of academic research confirming the positive effects of yoga on many health ailments. For example, in the majority of people yoga reduces anxiety and improves one's mood better than other forms of physical activities, such as taking a walk. This is also due to the increase in the quantity/level of gamma-aminobutyric acid (Stein 2010).

Explanations of exercises (asana) can be found on the Internet or even downloaded onto a mobile phone, so that one can always have with him/her both the instructions as well as a visualization of the exercises. So how do Poles view yoga? According to the studies of Kulmatycki and Burzyński (2008: 168) it seems that in Poland the preferred aspects of yoga are cognitive and recreational. Research on students underlines the importance of the cognitive aspect; they are looking for "something new" in yoga (Kulmatycki, Burzyński 1999). All of this reflects the great focus of the modern world on the body and bodily issues.

The thoughts included in this book are based on empirical qualitative research, and the rules accepted during the research concur with the qualitative sociology model (see: Introduction to Konecki, Chomczyński 2012). The basic rules of qualitative sociology are focused on understanding and explaining social phenomena. As John Dewey has stated:

[T] he world in which we immediately live, that in which we strive, succeed, and are defeated, is preeminently a qualitative world. What we act for, suffer, and

${ }^{5}$ Joga na Times Square [Yoga in Times Square], Rzeczpospolita, http://www.rp.pl/ artykul/187143,498263.html (accessed: 26.06.2010). 
enjoy are things in their qualitative determinations. This world forms the field of characteristic modes of thinking, characteristic in that thought is definitely regulated by qualitative considerations (Dewey 1931: 1).

The notion of commonality and common thinking connected with it (commonsense thinking) "that concerned with action and its consequences, whether undergone in enjoyment or suffering, is qualitative" (Dewey 1931: 1). In the epistemological layer, qualities have a subjective and psychological character, referring to the property of "outer objects" defined even with non-qualitative terms (ibid.).

Qualitative sociology is for both a perspective of a description, as well as understanding and sometimes even explaining reasons for social phenomenon through empirical research and analysis of individual and group experiences, the definition of the social world and interactions with the usage of qualitative methods of research or qualitative analysis, qualitative and/or quantitative data (Konecki, Chomczyński 2012: 10).

Connections between phenomena in this context can be understood only in reference to some interpretational units, such as in the context of qualitative unit as the general category becoming a yoga practitioner.

As described in this publication, research into the doing and practicing of yoga started with participant observation, carried out in one of the yoga schools in a major regional city from the years 2007 to 2011 , where the researcher took part in a class at least once a week. ${ }^{6}$ The researcher was also an observer of an outgoing yoga school on four occasions - during a week-long retreat in the mountains where, in seclusion, yoga was practiced for seven hours a day, and additionally there was meditation and mantra singing (in 2008), as well as during three-day-training trips where classes were also intensive, all day long with a 4-hour midday break (plus evening meditation; in 2008-2009). The researcher was then an active participant of the world which he researched. He dwelled together with the participants of the research and practiced hatha-yoga according to the teachers' recommendations. His psychological and physical sensing of the practice had an influence on his asking of questions during the research process. It can be said that during the group practice there was co-sensing with others of everything that was happening in the exercise room. This co-sensing

${ }^{6}$ Exercising yoga is understood by me as a physical approach to exercises with the usage of yoga positions (asana). Practising yoga is connected with the notion in which additional attention is given to the psychological effects and partially the spiritual effects connected with the performance of the exercises and gaining knowledge about the philosophy of yoga. 
was connected with the work on the body while performing hatha-yoga positions, the instructions given about performed positions, mutual meditation, or group mantra singing, which also causes both bodily and psychological sensations. During common practice, through the observation of one's feelings and undertaking different roles, interpersonal empathy appeared, which allowed the researcher to come to understand certain meanings ascribed to hatha-yoga by others. The feeling of the researcher himself was helpful in the interpretation of the data obtained from other sources that were basic sources in the described research (interviews and photographs and video recordings of hatha-yoga practices, observations). However, just the participation in the world of practicing would not, by itself, be enough for the researcher to understand the bodily and mental sensations appearing during the practice. Today, I can say that the practice itself and the imaged submersion in this world where the corporality is so important were essential. Hence, the observations here are directly connected with the experience of the world of the research participants. We used the symbolic interactionist theoretical approach to analyse empirical data (Becker 1982; Prus 1996; Clarke 1991; Strauss 1997).

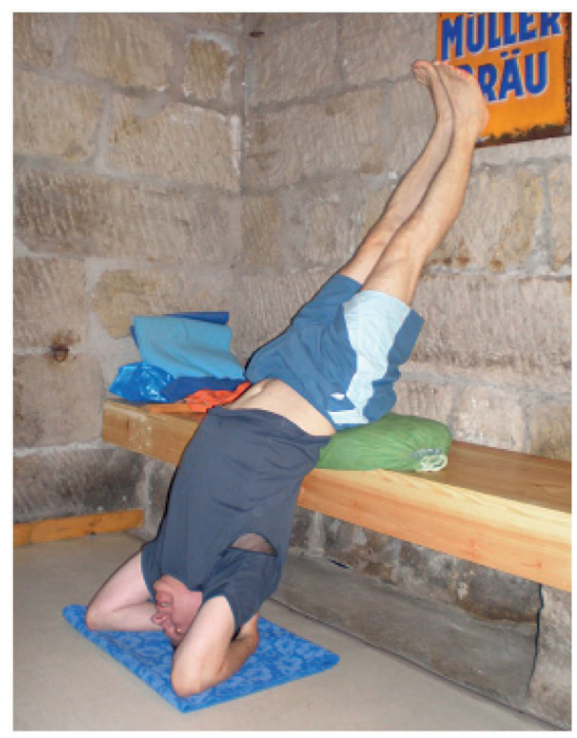

Fig. 1. Practice and participant observation by the author on the retreat in Pasterka, teacher: Michał Szczepanik from the Hatha-

-Yoga Centre in Łódź, 2008

(photo by Michał Szczepanik) 
During the research, in addition to 200 observational photographs, six video recordings of yoga practices were made. There were also 60 interviews, including thirteen narrative ones, 38 unstructured, and nine movie-elicited interviews. The interlocutors included pupils, students, working people, and yoga instructors, of both sexes. Interviews were done during the breaks of 2008 and 2009, with sociology students from the University of Lodz doing the interviews. The author has been running a participant observation from the year 2007 until the present. Additionally, 172 websites of yoga schools in 38 cities in Poland were analyzed in 2012 in order to examine the strategy and auto-presentational tactics of given schools.

The book begins, in chapter one, with introductions from the perspective of religious studies (Mircea Eliade) and from that of a great practitioner and guru of modern hatha-yoga (B. K. S. Iyengar). Both yoga connoisseurs have different approaches, the former points to many metaphysical and religious aspects of hatha-yoga, while the latter focuses rather on its role in improving health and, what follows, in subduing our everyday life. Yoga, through every day asana practice and a recommended way of nutrition (vegetarianism), results in calming of the mind and the elimination of negative emotions and consequently violence, and becomes a sort of "everyday religion." Modern hatha-yoga can thus be interpreted as para-religious, where there is no faith in supernatural forces, but an interest in ultimate concerns permeates. The problem of transcendence and the change of an individual is defined through the individual him/herself, so it seems that we are dealing here with a "privatized para-religion."

The second chapter describes the social world of yoga practice. Who is the participant of this world; what justifications are built and/ or used for participating in it; and what arenas are in it? Arenas are connected with whether yoga should be more of a physical or spiritual practice, who can be a yoga teacher (the problem of certification), and what is the origin of modern hatha-yoga. The participants of hatha-yoga often theorize it, looking for justifications for practicing but sometimes also pointing to its spiritual dimension and, in addition, they analyze and diagnose the state of Western culture. In chapter two points of intersection between the world of yoga practice and other worlds are shown (climbing, conventional and alternative medicine, business, pop-culture). In this chapter a wide historical panorama of the evolution of the current world of hatha-yoga practice is also shown in outline form.

The third chapter presents commonsense definitions of yoga that are constructed by the practitioners individually and for their own 
usages. These are often justifications for practicing yoga. The terms and meanings of what yoga is are often taken unknowingly from classical texts about yoga: through the media, yoga schools, classes, informal conversations with teachers, etc.

The fourth chapter deals with the issue of becoming a hatha-yoga practitioner. The process of becoming a practitioner is connected with practices concerning work on the body and its definition, and certain given forms of perception of the body and sensations coming from it. In this chapter, the phases of this process are described: 1) introductory phase - the construction of motives and first steps; 2) phase of fuller recognition of psychophysical effects and adequate meanings ascribed to them; 3) phase of fuller recognition of the spiritual aspects of hatha-yoga. The relation between mind and body becomes complicated in the moment of making a significant commitment to yoga practice and defining bodily practices as both mental and spiritual practices. Work on the body can change the Western perspective of defining the body as a material element of human existence (Cartesian vision) into a vision whereby it is treated as a spiritualized substance (Eastern philosophy vision), which is not always possible when we accept the assumptions of other religions (e.g., Catholicism). In such situations, the changes in the body and psyche have to be defined differently and there should be specific linguistic explanations (often within the scheme of constant language formulas) in order to reconcile the concepts of conventional religion with new spiritual experiences.

In the fifth chapter, research problems connected with the transfer of feelings and phenomena connected with corporality are taken into consideration. In the described research, coaching films on how to practice hatha-yoga were used, which in a realistic, objective way - yet somehow reflecting the physical reality of movements - showed how to perform some exercises (asanas) and sometimes what the consequences of performing a given exercise are for the organism, to see how the practitioners answer the general question: "What do I see and feel when I practice yoga?" In the interviews, based on the movies, interlocutors practicing yoga describe their feelings connected both with doing yoga, as well as the reception of the films. It is then described in what way the passing of knowledge about yoga practice from the aspect of feeling the body is constructed by the practitioners, and also the issue of the visual transfer of yoga practice is taken up.

The sixth chapter shows the work on emotions, which is done through specific physical practices using the body in order to obtain emotional stability, elimination of negative emotions, and achieve mental peace. Work on emotions is shown as an internalizing ritual 
of "modern religion," which is most commonly unseen to an outside observer, and sometimes even to the one working on emotions. The work on emotions in hatha-yoga then has an initiating character, i.e. whether there is a visible change in the individual certified by his/her new interpretation of his/her own emotions and self-feeling. The aim of this part of publication is to show what the work on emotions in yoga practice is, as well as an attempt to interpret the phenomenon in the light of the processes happening in modern Western societies.

In the seventh chapter I deal with the role of the teacher in the yoga-practice role, which is extremely important in the development of the practitioner. According to one of the yoga gurus - B. K. S. Iyengar - the pupil has to have endless trust in his or her teacher. While this is to some extent an idealized conception of the pupil-master relation, nevertheless according to practitioners the teacher is essential in the practice of yoga, as an instructor in physical exercises, and sometimes even as a life guide.

For many practitioners, hatha-yoga has become a style of life - a style in which attention is paid to one's fitness and mental state and sometimes to one's own spiritual condition. Practitioners advanced in practice are often faithful to their teachers and to the schools which they attend. Their everyday routine is subdued to yoga, which changed their identity and helps in coping with everyday life as often described by the values of the Western world. From this clash of two cultures comes what we call "modern hatha-yoga," a very popular form of rest, physical exercises, health practices, and even spiritual practice. Everyone can choose their own interpretation from an array of interpretations, and create for their private usage their own definition of the practice. 


\section{CHAPTER 1 \\ Philosophy and hatha-yoga practice Para-religious aspects of hatha-yoga}

\subsection{Introduction}

The explanation of what hatha-yoga is seems a difficult task for a sociologist. I am even more aware, since practicing yoga and observing yoga practitioners and listening to their explanations, of the wide array of common definitions of yoga, as well as the many types of yoga. People who practice different types of yoga usually treat the given type which they practice as the source, and even sometimes as the one and only true source, of that which can be called yoga. While we will deal with the problem of a common definition of yoga by practitioners in Chapter 3, at this point we will examine how yoga is defined by a religious historian and theologian, Mircea Eliade, and a practitioner and theoretician of yoga, Bellur Krishnamachar Sundararaja Iyengar, who is a spiritual guardian, teacher, and master (guru) in his own line of yoga teaching, often called Iyengar's School.

Iyengar has created a very buoyant B. K. S. Iyengar's Institute of Yoga in Pune, India, which educates teachers propagating hatha-yoga around the world. Iyengar (December 14, 1918-August 20, 2014) wrote several books that explain his philosophy of yoga and also gave instructions for executing certain asanas (positions) and breathing practices (pranayama). During his youth, Iyengar cured himself from many physical ailments and sicknesses through yoga practices (2005a). Maybe this biographical fact established his strong tendency to accent physicality and physical exercises in his concept of hatha-yoga. $\mathrm{He}$ is often accused of having a physical approach to yoga. However, in order to level this tendency in his work and in the perception of his school, he wrote a book after 70 years of practice, Light on Life, which shows other elements of yoga, accenting more the spirituality of this philosophy, and also translated and explained Patanjali's sutras, which express the essence of yoga and show its spiritual character (Iyengar 2002a; 2005a).

Iyengar was a great propagator of hatha-yoga in the Western world. He visited Great Britain for the first time in 1954 at the 
invitation of Yehudi Menuhin, and in the beginning he taught a small group of friends and friends of the musician (Hasselle-Newcombe 2005: 306). From this moment the number of yoga enthusiasts became ever greater, and now in Great Britain there are about a half million people practicing yoga. The dominant variant of yoga practiced there is hatha-yoga (ibid.: 305, 307).

It is extremely difficult to go outside of the original definitions given by specialists and/or masters. For this reason I cite them in order to give formal definitions of yoga and its socially conditioned practices. I will, however, try to interpret them from a sociological point of view.

\subsection{Yoga - the perspectives of a religious studies scholar and the perspective of yoga practitioner}

Mircea Eliade is a religious studies scholar and a scientist, not a yoga practitioner. He shows us the source topics that created the philosophy of yoga in the Indian context of religion. In traditional Indian thought, the problem of freeing a person from earthly constraints was very crucial. In this tradition the philosophy of yoga, as any other philosophy, is supposed to reach the truth, and the truth frees the person. The "truth" is equal here with liberation, i.e. achieving absolute freedom. Liberation is about moving into a different kind of existence, transcendental towards human existence. It is about some results of a mystical nature, a rebirth to an unconditioned existence (emphasis by M. E.). This is liberation, absolute freedom (Eliade 1997: 20). M. Eliade describes what yoga is as follow:

\footnotetext{
Etymologically, it derives from the stem yuj - meaning to tie, to connect, to harness, to tame which corresponds to the Latin iungere and iugum (English yoke). The word yoga is used to describe any technique of asceticism and any method of meditation [emphasis by M. E.]. There is classic yoga, which is also a philosophical system introduced in the famous Pantanjali treatise Yogasutrani. However, apart from this classic yoga, there are also numerous forms of folk yoga not encompassed by the system, as well as non-dual-brahman yoga (e.g., Buddhist, Jainistic), and mostly yoga with a magical structure or mystical structure (Eliade 1997: 21).
}

Yoga embraces a disconnection from the material world. This is possible by disciplining oneself, mind concentration, and in different mystical forms of yoga by turning oneself to a deity.

"Yoga is characterized not only by the practical side (emphasis by M. E.) but also by the initiation structure (emphasis M. E.). Nobody learns yoga on their own, directions from the guru are needed" 
(Eliade 1997: 21). The initiation of yoga is based also on the fact that a yogi moves away from the evanescent world. The yogi moves away from the layman condition and "wants to die to this world. We assist in the death after which there is rebirth into a different way of existence which is freedom" (ibid.: 22). Yoga is an initiation system because the yogi is somehow reborn. Brahmanism, as opposed to the Buddhist and Jainist forms of yoga, accepts yoga according to the Pantanjali system. Eliade writes that yoga has a theistic character as it recognizes a highest god (Ishvara), as well as soteriological, salvation is based on freeing oneself from the cycle of renewed incarnations (moksha). In yoga, asceticism and meditation techniques are needed in order to gain freedom.

Below I extensively quote certain characteristics and elements of yoga techniques in order to emphasize that yoga, understood and practiced in a classical sense, changes the practitioners' way of perceiving the world and acting within the world. It is not only an interaction on the physical surface of a person.

The starting point of the yogic meditation is focusing on any given thing, whether it is a physical object (a point between colors, the tip of your nose, some lucent thing, etc.), thoughts, metaphysical truth about Ishvara. Such strong and constant focus is called ekagrata (meaning in one point) and it can be gained by splitting the mental stream (sarvartha - split, dispersed attention) [Yogasutrani III, 11]. This is the right definition of yoga technique: jogah ćittavrittinirodhah [Yogasutrani I, 2]. That is why the practicing of yoga is started from ekagrata, which builds a dam against the stream of thoughts being some sort of mental blockade, a homogenous continuum. Practicing ekagrata is directed towards the controlling of two powers creating the stream of thoughts: sensual activity (indrija), as well as work of the subconsciousness (sanskara). A yogi can stop the continuum of consciousness as he wants. In other words, he can at any time and any place focus on one object stopping his reaction to any other sensual or memory stimuli. Thanks to ekagrata one gains a real will, which is a possibility of working on any biological and mental field activity (Eliade 2009: 80-81).

Ekagrata is gained by physical exercises, so physicality and physiognomy are very important here. The practice of yoga is hence connected with physical and spiritual exercises (called anga - elements) that need to be learned in order to get to ekagrata and then to the highest state of focus, called samadhi. Elements of yoga are: 1) moral obligations (yama); 2) ascetic practice (niyama); 3) stature and positions of the body (asana); 4) breathing rhythm (pranayama); 5) freeing of the senses from the domination of outer objects (pratyahara); 6) concentration (dharana); 7) yogic meditation (dhjana); 8) full freedom (samadhi) (Eliade 2009: 82). 
Classic yoga is closely connected to ascetic practice. According to Eliade, niyama is an ascetic practice. Tapas (asceticism) is about coping with adversities such as hunger or thirst, heat or cold, cravings for standing or sitting, on being silent (kastha mauna), and the lack of facial expressions that would give away thoughts or feelings (akara mauna). Vachaspati Misra specifies it: "the lack of facial expressions giving away deep secrets of the spirit, it is a show of control over oneself, as thoughts are not shared with just anybody" (see: Eliade 1997: 85).

So even today, when those practicing yoga hear from their teachers while doing asana "calm face, keep a calm face," they are somewhat practicing tapas, even though in most cases they do not even know this, nor do they know the name of this ascetic practice. ${ }^{1}$

The third element of yoga, after yama and niyama, consists solely of a yoga technique called asana. Asana belongs to Indian ascetic techniques. It is mentioned in the Upanishads, in Vedic literature, in Mahabharata and Puranas. Asana is a yoga statue that is supposed to be still and comfortable (Eliade 2009: 87, 89). It is, however, an introduction to real yoga practices. This is also so claimed by the yoga practitioner Pattabhi Jois (2010). Thanks to asana practice, one gains a physical strength, which then leads to improvement in health. Here work on the body is a way to achieve a different state of existence. Asana is the first concrete step to abolishing the conditioning of the human existence. On the level of the body, asana is ekagrata, focusing on one point. The resignation from movement while staying in asana, resignation from stormy streams of conscious states (ekagrata), is only the beginning of a whole series of different types of resignations (Eliade 2009: 90).

A different yoga technique, also very important to gaining the state of resignation from the evanescent world, is pranayama. Resignation from the evanescent world is here connected with the resignation from breathing; Pantanjali is about stopping breathing. In the beginning, it is about achieving rhythmical and slow breathing. By achieving the breath rhythm of a sleeping person, a yogi can penetrate (without resigning from being awake) states of consciousness typical for sleeping (ibid.: 92). Eliade distinguishes four kinds of consciousness that are present in Indian psychology: daily consciousness, sleep consciousness and night dreams, sleep consciousness without night dreams, and cataleptic consciousness. Experiencing these four types of consciousnesses is possible after a long time of practicing. In general, thanks to the pranayama exercises, we can first gain a continuous consciousness, which allows for the execution of yoga meditation.

\footnotetext{
${ }^{1}$ Data gathered from participant observation of the researcher.
} 
The religious character of yoga is seen vividly in the yogi's withdrawal from evanescent life in asana positions and breath control (pranayama). A yogi exists in the world like a plant. Looked at from another point of view, asana and ekagrata copy some godly archetype. Yoga positions also have a religious quality. The discarding of human existence, which is practicing yoga, has a religious dimension in the sense that a yogi copies the way of Ishvara's existence - his stillness and focus on himself. In other yoga variations asana and ekagrata can gain a religious dimension simply because thanks to them a yogi becomes a live statue (ibid.: 100). This rejection of human existence and complete abandonment of the world in soteriological and contemplational religions is also underlined by Max Weber. Avoiding purposeful, rational action is the initial condition to gaining the state of grace (Weber 1984: 226, 228).

Breath suspension allows for the attainment of concentration ( $d$ harana). This state of focusing can be verified by the possibility of a withdrawal (pratyahara) or disconnection from the senses. Sensual activity is then not dependent on outer objects. A yogi starts practicing a triple technique called samyama. This term refers to the three last stages of yoga practise: concentration which is ekagrata, the focusing of the stream of thoughts on one point (dharana), exact meditation where we deal with the stream of united thoughts (dhjana), and enstasy, which is a full concentration, stagnation, connection with the object of contemplation (samadhi). These three practices are subtle and sometimes transfer from dharana to meditation and then to samadhi, which is unseen and very quick for the practitioner. Meditation and the state achieved by it is very difficult to describe, although Eliade writes clearly about it. This difficulty is also experienced by many meditating practitioners. Nevertheless, the aims are clearly stated - penetration of the essence of matter and union with the reality (ibid.: 106).

When it comes to the religious aspects of yoga, one needs to refer to the role of God in yoga. It is not the role of God interfering with the yogi's life. He/she is outside the evanescent world, he/she cannot be asked about anything or be begged for anything. God, mentioned by Pantanjali, is rather the god of yogis. He/she may help only a yogi, a man who has chosen yoga (ibid.: 107). Ishvara is yogi's archetype. In yoga sutras, yoga techniques are emphasized more than the role of Ishvara in gaining samadhi. Ishvara is only an element necessary for exercises (see: Pantanjali sutras in: Iyengar 2002a).

Samadhi is the final stage of a yogi's development. A yogi does not distinguish between the act and the object of meditation. It is not a hypnotic trance. Samadhi is a state in which the self is shown (Puru- 
sha) (Eliade 1997: 116). Enstasy with its qualities (samprajnata) has several stages (Eliade 2008: 118-119).

The practice of samyama gives a yogi some occult skills, for example, entering into others or recognizing their states of consciousness, making oneself invisible to others, the power to know the time and day of one's death, possessing inhuman physical strength, as well as knowing the subtle things, etc. This does not happen thanks to supernatural powers, but thanks to the powers and skills that a yogi gains through samyama.

A yogi reaches freedom; he becomes somewhat dead while alive. This is a paradox of a yogi's existence. He is freed from evanescent and material life, but he is still part of it. He is on the borderline where the evanescent world meets the spiritual, the sacred meets the profane.

It needs to be underlined that yoga is not a humanistic philosophy. It contradicts everything that is human in the evanescent sense of being human. Yoga requires solitude and absolute celibacy (ibid.: 130). Yoga is in this sense religious, as it discards the profane in the name of sacredness, so achieving independence from the material and social world, freedom and power outside this world - several of the important characteristics of sacredness are accented here. There is a reversal of all human values. Samadhi is the act of real transcendence, albeit paradoxical. This state leaves the being, but at the same time it fills it completely. The initiatory character of yoga is based on the fact that we leave this world in order to be born again, however, not for this world but for the primal, sacred world, full of freedom and consciousness.

\section{$* * *$}

B. K. S. Iyengar is known to us as a great practitioner and propagator of hatha-yoga. In his books and teachings, he concentrates mainly on physical and breathing exercises. His books have also been published in Western languages for Western audiences.

His yoga is often called 'physical yoga' inasmuch as it concentrates on physical exercises and the body. However, at the beginning of his book, Illustrated Light on Yoga, which is a formal description of hatha-yoga positions, breathing practices (pranayama), and the health results coming from practicing certain asanas, Iyengar gives a definition of yoga: 
[T] he word Yoga is derived from the Sanskrit root yui, meaning to bind, join, attach, and yoke to direct and concentrate one's attention on, to use and apply. It also means union and communion. It is the true union of our will with the will of God (Iyengar 2005a: 1).

This definition at the beginning of a book describing physical exercises may seem surprising. One can vividly see religious aspects (e.g., the concept of God) that are most commonly not absorbed in a direct and open way by practitioners in the Western context. The connection of the will of human being with God is common for many religions, the difference being that, for example, in hatha-yoga, the connection is done by work on the body, ultimately by corporeality.

Iyengar, in the introduction to the book concerning the positions of yoga, clearly writes: "Yoga is one of the six orthodox systems of Indian philosophy. It was collated, co-ordinated, and systematized by Pantanjali in his classic work, The Yoga Sutras, which consist of 185 terse aphorisms" (Iyengar 2005a: 1; see also Iyengar 2002a).

In Indian thought, everything is permeated by the Supreme Universal spirit (Paramatma or God) of which the individual human spirit (jivatma) is a part. The system of yoga is so called because it teaches the means by which the jivatma can be united to, or be in communion with Paramatma and secure liberation (moksha). One who follows the path of Yoga is Yogi or Yogin (Iyengar 2005a: 1).

Yoga was mentioned in the classic books of Hindi religions (as was emphasized earlier by Eliade). The Bhagavadgita also gives a different description of yoga, putting emphasis on karma (yoga of the deed). It is said there:

\footnotetext{
Thy motive, not the fruit which comes from them.

And live in action! Labor! Make thine acts

Thy piety, casting all self aside, contemning again and merit; equable

In good or evil: equability

Is Yog, is piety! (Bhagavadgita 1993: 11).
}

The Kathopanishad describes Yoga thus: "When the senses are stilled, when the mind is at rest, when the intellect wavers not - then, say the wise, is reached the highest stage. This steady control of the senses and mind has been defined as Yoga. He who attains it is free from delusions" (as cited in Iyengar 2005a: 2). Thus we could say that Iyengar, even in a book describing in a formal way the bodily aspects of yoga (the exercises and their health effects), concentrates on the most outer layer of the body (annamaya kosha), in the introduction, however, he emphasizes the spiritual aspects of yoga. What is very important 
in Iyengar's statement, and so often repeated by him, finds expression in the following quote:

In the second aphorism of the first chapter of The Yoga Sutras, Pantanjali describes Yoga as chitta-vrtti-nirodhah. It may be translated as restraint (nirodhah) of mental (chitta) modification (vrtti) or as suppression (nirodhah) of the fluctuations (vrtti) of consciousness (chitta) (Iyengar 2005a: 2).

Work on the mind is a vital element of the practice of yoga and is done not only at the level of the physical body.

The mind (chitta) consists of three spheres: a) manas (individual mind) - a sphere having the ability of attention, choice, and rejection; it is a changeable, non-adjudging power of mind; b) buddhi (intellect, sense) - capability of adjudging, determining differences between things; c) ahamkara (ego, literally creating the feeling of "I") - mental power that enables the creation of the statement "I know" (Iyengar 2005a: 2).

Vrtti derives from the Sanskrit vrt, which means agitation, twisting, wriggling. Hence, this is about stopping the agitation of the mind on three levels, and the level "I" is very important, which is visible in eternal agitation, especially when one fights against real or imaginary attacks on oneself, for example, on beliefs preached by an individual. Calming down the mind is connected with many prohibitions. For example, a different guru-yogi, Sri Pattabhi Jois, is more rigorous than B. K. S. Iyengar and writes even about the prohibition of vain speech:

Similarly, it is not good to talk too much. By talking too much, the power inherent in the tongue decreases and the power of speech is destroyed. When the power of speech is destroyed, our words, too, lose their power, and whatever we utter has no value in society at all. Talk of spiritual matters, however, increases the tongue's power, and is thus helpful to the world. But speech related to mundane matters destroys the power of the tongue, and shortens our life spans. The shastrakaras have reflected on and described this fact, so it is better if man follows their path (Pattabhi 2010: 25).

Iyengar, being a yoga practitioner and an actively working teacher, rarely mentions asceticism in yoga practice (as well as rituals in yoga practice). This is probably due to the fact that yoga would not be accepted in the modern world, dominated by the Western culture, if its main element was asceticism, as is suggested in niyama (see the earlier thoughts of Eliade). Clearly, Iyengar adapts yoga to the current conditions of its practicing, hence, towards the attitudes of the addressees of the idea of yoga, their habits and conditions of life, and the market in which the ideas of yoga are being sold in different packages, none of 
which talk about the need for asceticism and sexual abstinence or the need for withdrawal from earthly life. In his books, one can see a greater concentration on the therapeutic usages of yoga and on emotions, whether it concerns the liquidation of negative emotions, the liquidation of illnesses of the body, or the improvement of physical and health conditions. However, Iyengar also points out the roots of hatha-yoga in the traditional Indian philosophical culture and yoga's reference to religious systems. Iyengar's yoga is directed towards everyone, without the need to move away from people close to you or from society in order to focus on ascetic practices and yoga techniques which are separate from the typical practices of a given society. This focus on earthly life is visible in his treatment of yoga as an element of everyday life in modern society, sometimes having a vivid spiritual aspect, sometimes physical and/or psycho-emotional.

\subsection{Yoga and society - "religion of everyday life"}

Yoga is often perceived as an individual form of spirituality. This is true not only of those who practice it, but also of those who criticize this form of body and soul practice. Contrary to common beliefs, yoga is not directed only towards individual development (physical, mental, and spiritual), but also to the development of society, peace, and happiness. Those who have solved their emotional problems are more empathic towards others, more understanding, less judgmental, and give examples of good behavior. Yoga also improves society or the community because it depends on and fosters the development of individuals (Iyengar 2002b: 10-12). Individual forms of spirituality become, paradoxically, a splendid form of socializing individuals and creating pro-society behaviors.

Violence in society can be liquidated when people liquidate it in themselves. According to Iyengar, "One who is an undisciplined man is an irreligious person. One who is disciplined is a religious person... Health is religious. Ill-health is irreligious" (ibid.: 11). The last sentence shows the connection between practicing yoga, health, and religion in the view of Iyengar. Many sociologists emphasize that the interest in personal development, focusing on health, physical exercises and vegetarianism in modern Western society, has a religious or para-religious character. There is a need for and a practice of personality change and a change of one's sense of life (Greil, Robbins 1994; see also Grail, Rudy 1984). "The methods of spiritual development are given for the evolution of individuals thorough the world" (Iyengar 2002b: 16). 
Priorities in one's individual life change when discipline appears in yoga practice and in the life of an individual. Yoga becomes the religion of everyday life. It is not a showy religion filled with rituals and prayers, nor with withdrawal from everyday life. At some point, life, when we are fully aware of what is happening to us, becomes a religion; life becomes a unity and the individual becomes a whole. Iyengar gives examples pertaining to everyday life in order to show that yoga is an omnipresent philosophy and practice that in the end becomes the religion of everyday life.

"Religious life is not withdrawal from the everyday world... When they invite me to drink, if I say, 'No I am not interested,' they will laugh at me. So I say, 'I will come. Give me fruit juice and you take the alcohol...'" (ibid.: 11). Iyengar emphasizes vividly that one need not withdraw from everyday life in order to apply the rules of yoga. On the contrary, dealing with everyday life gives us the opportunity to practice the rules of yoga directly in our life.

According to Iyengar, we practice yoga for the society. A similar attitude characterizes another propagator of hatha-yoga - Sri Pattabhi Jois in ashtanga yoga. Yoga is supposed to prevent sickness in everyday life and allow for control of the mind:

If the limbs and sub-limbs of yama and niyama are to be practiced, then steps should be taken to ensure that one does not fall victim to disease, obligation, or poverty. For when a person becomes ill, his mind cannot be steady, nor can he do any work. Therefore, the body, sense organs, and mind must be stabilized to prevent obstacles, such as disease, from occurring. To bring the body and sense organs under control, the asanas, or postures, should first be studied and practiced (Pattabhi 2010: 16).

Those most connected with everyday life are the bans (yama) and orders (niyama) from the eight-scale yoga path described by Pantanjali in The Yoga Sutras in second chapter (Iyengar 2002a). Eliade states that: "Two first groups of practice, yama and niyama, are of course an introductory stage to every asceticism. Hence, they have no feature common only to yoga" (Eliade 2009: 82). Niyama, for Eliade, is a series of ascetic, body and mental exercises (ibid.: 84), while Iyengar does not treat niyama as ascetic exercises. In general, he avoids the notion of asceticism, although it is not always possible in the description of yoga. The notion of asceticism could discourage many modern lovers of hatha-yoga from practising asanas. Yamas are universal prohibitions (ethical disciplines) concerning all people: ahimsha (prohibition against killing), satya (truth, not lying), asteya (not stealing), brahmacharya (sexual abstinence), aparigraha (non-possessiveness). 
These commandments are the rules of morality for society and the individual, which, if not obeyed, bring chaos, violence, untruth, stealing, dissipation, and covetousness. The roots of these evils are the emotions of greed, desire, and attachment, which may be mild, medium, and excessive. They only bring pain and ignorance (Iyengar 2005a: 13).

The second step in the path of yoga, referring to the life of an individual in society, is the step of niyamas. These are rules used in individual practice: saucha (cleanliness, both outer and inner), santosa (contentment), tapas (ardor, asceticism), svadhyaya (knowing, self-inquiry, learning about one's own ego), and Ishvara pranidhana (devotion to the Lord, sacrifice of one's own deeds and thoughts to the Lord). This last rule is, to be honest, the most religious in the yoga system (in our Western understanding of religion). Here one finds bhakti (glorification, adoration of the highest), if we acknowledge worshipping of the highest as one of the constitutive features of the religion: "When the waters of bhakti (adoration) are made to flow through the turbines of the mind, the result is mental power, spiritual illumination. While mere physical strength without bhakti is lethal, mere adoration without strength of character is like an opiate" (Iyengar 2005a: 21; see also Eliade 2009: 84-85).

Is yoga according to Iyengar a religion? In writing about svadhyaya order (learning about oneself), Iyengar states that it is not a religion:

Philology is not a language, but the science of languages, the study of which will enable the student to learn his own language better. Similarly, Yoga is not a religion by itself. It is a science of religions, the study of which enables a sadhaka [student - K. T. K.] to better to appreciate his own faith (Iyengar 2005a: 21).

In this way, Iyengar does not discourage followers of other religions from practicing yoga. He does not build any barriers or borders for yoga by use of an order employing clear rules of a religious system. However, when analyzing many previous quotations one can have doubts about the statement that yoga is not a religion. Often in descriptions of yoga one encounters statements about God and godliness ("By svadhyaya the sadhaka understands the nature of his soul and gains communion with the divine," "Without tapas the mind cannot reach up to the Lord," "The name of the Lord is like the sun, dispelling all darkness" [Iyengar 2005a: 20-21]). According to me, it seems that Iyengar, despite his assurances that yoga is not a religion, treats yoga as a para-religious meta-system that can be adapted to any system of beliefs where there is a God (or Gods) or a Highest Spirit. It needs to be mentioned here that faith in the highest spirit is not understood 
here as faith in a transcendental God in the way that it is understood in Christianity whereby a Christian fully surrenders to God, but rather faith in an immanent God that is somewhat a God inside the individual and the world (Davie 2007: 165).

Although many religions exert pressure on everyday life connected with prohibitions and orders pertaining to our fellow members, if the rules are not built on the basis of a direct relation with a personal God and surrendering to God and/or fear of God, then a religion of this type must concentrate on everyday life and on everyday behaviors in relationships with others (not only humans, but also animals and the natural environment). Though God can appear in the background, all practices and consciousness of being is here and now. However, yoga is not focused on devotion and adoration of the Lord nor on emotional surrendering to the Lord (bhakti), as often expressed by rituals towards the highest being, although this aspect of practicing yoga may still be very important to many persons. The practice of yoga is something different than ritualistic devotion; it is a development of the subject and self-research into one's own nature and true self. This thesis, and knowledge about its influence on everyday life, are contained in The Yoga Sutras and in the interpretations of yoga teachers. This especially refers to Iyengar's interpretation. It accentuates one's own self, and thus the unity of a person consisting of body, mind and soul, as well as self-knowing, thus answering the question: "Who am I?" and how to cure the ego, which is characteristic for so-called "individual forms of spirituality" or the "New Age movement" (Davie 2007: 164).

The spirituality of modern yoga (hatha-yoga) is also emphasized in suggestions about the way of eating. Vegetarianism limits violence. The way in which one eats influences one's way of thinking: "The West have now changed their diet to vegetarianism, as vegetarianism leads to a minimum of violence" (Iyengar 2002b: 13). The way of eating is important for peace of mind. The mind is a product of the food one eats. However, if health is the only aim of practicing yoga, then vegetarianism is not necessary (ibid.: 13). It can be seen that vegetarianism, in the version of yoga modernized by Iyengar, is not a necessary condition to practicing hatha-yoga. However, it is necessary when we want to include spiritual elements into the practice.

The issue of faith in God, in the case of a modern human who practices yoga, is solved by Iyengar in a particular way. We do not have to believe in God. It is enough if we believe in the existence of ourselves. If we believe in our own existence, then we want to perfect ourselves and improve the quality of our life. If we act in this way, it will push us in the spiritual direction (ibid.: 37). Faith is a subjective experience for 
oneself, and faith in God is secondary. In Iyengar's approach, self-perfection and individual development are important. There are values of para-religion in this: "Believing in God is secondary. And as you are living, you want to improve. You want to be better than you are. This is the golden vitamin which will enable you to progress" (ibid.: 37). So, development and self-perfection are here recurring and leading motives of yoga practice.

All of these formal features of yoga make us treat it, from a sociological point of view, as a para-religion and earthly religion, where self-development gives peace, and the knowing of one's true self and moral rules (for example, not hurting others, as shown in vegetarianism) allow the individual to build social harmony and contribute to the common good. All of these features are what sociologists of religion call "individual forms of spirituality."

\section{Yoga - adjustment to everyday life}

Iyengar's interpretation, or re-interpretation to be correct, of yoga rules results in its stronger adjustment to the conditions of everyday life and human attitudes in the modern world. For example, he treats the fourth aspect of yama, brahmacharya (sexual abstinence) in a less rigorous way than is suggested in the original texts and in examples taken from the lives of saintly persons in India:

It needs to be mentioned though that sexual abstinence (brahmacharya) is not only about keeping yourself away from sexual acts, but about burning out the sole lust. Instinct cannot remain in the shadows, sleeping in the subconsciousness; it also cannot be sublimated like in the case of mystiques, but simply destroyed, rooted out from the consciousness and senses... Pantanjali, of course, represents the ascetic tradition par excellence (Eliade 2009: 84).

Iyengar demonstrates a certain distance to the strict traditions of asceticism and celibacy, even though Pantanjali is a supporter of asceticism and moral rigor:

This does not mean that the philosophy is meant only for celibates. Brahmacharya has little to do with whether one is bachelor or married and living the life of a householder. One has to develop the higher aspects of Brahmacharya in one's daily living. It is not necessary for one's salvation to stay unmarried and without a house. On the contrary, all the smrtis (codes of law) recommend marriage. Without experiencing human love and happiness, it is not possible to know divine love. Almost all the yogis or sages of old in India were married men with families of their own. They did not shirk their social or moral responsibilities. Marriage and parenthood are no bar to the knowledge of divine love, happiness, and union with the Supreme Soul (Iyengar 2005a: 16-17). 
According to Iyengar, it is possible to practice yoga and have a family at the same time. The practice should be incorporated in everyday life: "Remaining in the midst of the family, always doing the duties of the householder, he who is free from merits and demerits has restrained his senses, attains salvation" (Iyengar 2005a: 17). A similar approach, minimizing the rigors of brahmacharya, is presented by a different guru of modern yoga, Sri Pattabhi Jois:

Maintaining brahmacharya nowadays is difficult because there are so many things that attract the mind in different directions, such as theaters, pleasure houses, restaurants, and the like. The preservation of brahmacharya is thus an uphill task. Now, a question arises: If we cannot maintain brahmacharya, does it not amount to saying that yoga is impossible for us? No, a man can achieve some degree of brahmacharya. If he is to achieve it, however, he must avoid the following as much as possible: mixing with vulgar people; going to crowded areas for recreation; reading vulgar books which disturb the mind; going to theaters and restaurants; and conversing secretly with strangers of the opposite sex. If these are avoided, brahmacharya can be preserved in part (Pattabhi 2010: 8).

However, these limitations on the rigor of brahmacharya are connected with abiding by a set of rules for sexual intercourse: intercourse only with one's wife during the night, the length of the day and night is defined by the way of breathing; the menstrual cycle of a woman should also be taken into consideration and intercourse should happen between the $4^{\text {th }}$ and the $16^{\text {th }}$ day of the cycle (Pattabhi 2010: 11).

We should also remember that Iyengar's family belongs to orthodox Vaishnavites, which can have specific meaning for Iyengar's teaching and his descriptions of the spiritual aspects of yoga (Hasselle-Newcombe 2005: 308). He very often quotes Bhagavadgita (Hasselle-Newcome 2005). The spirituality of yoga, and even the religious aspects of hatha-yoga, are emphasized by him on numerous occasions. The adjustment of yoga to the everyday life of a modern man is also about accenting the health and therapeutic effects of hatha-yoga: "Yoga helps to maintain the defensive strength at the minimum level, and that is what is known as health" (Iyengar 2002b: 33). One finds, next to the descriptions of all asanas in the book Illustrated Light on Yoga, also descriptions of the health effects of given asanas. Thus, it is not strange that hatha-yoga, according to Iyengar's school, has garnered such a wide interest in society, where the interest in physical health and physical exercises are helping to keep it widespread.

Health is one of the most important values in Polish society. Maintaining good health is important to $97 \%$ of Poles, even if it does not apply to their everyday life (Boguszewski 2010). About one third of Poles 
(33\%) are worried about their mental health and the reasons they give for that are harmful life conditions, such as unemployment (62\%), family crisis (53\%), abuse of alcohol and drugs (46\%), poverty (37\%), an insecure future $(24 \%)$, the fast pace of life $(20 \%)$, and bad relations among people (17\%) (Wciórka, Wciórka 2008). It is visible that we look for threats to our mental health in material conditions. All therapeutic and healing practices can become an object of interest in the context of such a social atmosphere. Yoga, in this context, can certainly be chosen as a practice aimed at improving our health. Iyengar points to the positive health consequences of practicing yoga (2005a), and the more physically-oriented guru of hatha-yoga, Sri Pattabhi Jois, points to the same thing (2010). Following a description of how to do a given asana, he also lists the health effects coming from carrying it out. The health results of yoga are also underscored in the advertisements of yoga schools and auto-presentations on Internet websites.

\subsection{Conclusions}

Classical yoga has a religious character because, as the religious study scholar Mircea Eliade has shown, it has an initiative character; the individual is freed from the material world and stops being reborn in new incarnations. Yoga allows one to discard what is secular and profane and to gain what is sacred. In this sense it is not a humanistic philosophy, as everyday life is here not important to achieving full freedom. What is important is the practice of yoga and discarding this life.

Hatha-yoga practice, as defined by Western culture, surely has some features of the definition of so-called "para-religion." Iyengar redefines given rules of yoga in order to transplant it into the soil of Western culture; for example, health for Iyengar has a religious aspect.

Para-religious phenomena are connected with an interest in the final and existential aspects in the life of a man, but not with beliefs in supernatural powers. The para-religious approach is present in, for example, some psychotherapies or in various corporate cultures where work on the meaning of life and individual identity are undertaken (Greil, Robbins 1994; also see Greil, Rudy 1984, who write about socalled "identity transformation organizations"). These organizations, whether they ascribe to themselves features of religious organizations or not, show some features of religions and religious practices. Yoga practice in the Western context can be treated as a privatized para-re- 
ligion, where the individual defines what is important for him/her on the basis of the "ultimate concerns" one is working on, for example, identity or understanding the sense of life and its aim.

Some practices can be treated as a quasi-religion because meaning is ascribed to supernatural powers, but this is not a religion as religion is commonly thought of, as it does not have an institutional sense (it does not have Church accepted by society). Quasi-religions include, for example: occultism, New Age, magic, spiritualism, astrology, etc.) (Greil, Robbins 1994). Quasi-religion oscillates between the sacred and profane. Yoga practice can also meet these definitional conditions because many who practice physical exercises called asana (profane) believe that God shows himself in the soul (sacred), the existence of which can be seen in the body after many years of practice, including meditation (sacred and profane). However, the choice and interpretation of the aforementioned elements are highly privatized and individual.

Whether hatha-yoga is a para-religion or a quasi-religion will be decided by whether the practitioners ascribe some meaning to supernatural powers or not. If, for example, one considers supernatural powers the work of Hindu Gods or cosmic energy (prana), then hatha-yoga will be a quasi-religion. If we do not take into consideration these elements, then hatha-yoga solves existential problems and changes the identity of an individual, which then is a para-religion. All of these statements about privatizing spirituality and religion become religiousness as autonomically described by the individual (Knoblauch 2006; Luckmann 2006). However, it should be kept in mind that religion is a social, and not purely psychological, creation (Prus 2009: 124-125).

This privatization aspect seems, according to me, to be strongly connected with individual para-religiousness. Thomas Luckmann writes about an individual religiousness that develops in the family bosom, and this often applies to vividly separate religious systems. We are interested in the individual construction of individuals' own beliefs, often not in agreement with what their closest family has to offer. Friends, neighbors, and members of groups created at work and, as Luckmann states, interest groups, create the private universes of meaning and take on the function of significant others (Luckmann 1967). Even though private choices show the individualization of religion, the network of social relationships gives support to the individual.

Yoga can here represent a case supporting the thesis of the subjectivization of modern society (Heelas et al. 2004). Subjectivization means a concentration on one's individual life; a larger meaning is ascribed to personally experiencing the world, caring about personal health, and social relations. Modern Western culture has turned toward 
the subjective on a mass scale. Spiritual forms that subscribe to this focus will, of course, develop and gain popularity. Empirical research confirms this thesis (Heelas et al. 2004; see also Lynch 2007: 119). The spiritualities of subjective life concentrate on one's well-being in this life, i.e. more on the health of an individual than on life after death or eternal life. The idea of outer God is discarded. An individual and his or her needs, personal experiences, and freedom and self-expression are given more value. Heelas called this phenomenon "humanistic expressivism" (2000). Humanistic expressivism naturally takes, in the Western context, inspirations (for example when it comes to language, the lexicon needed to interpret phenomena from an individual level) from religion, philosophy, and the traditions of the West, but also from mysticism, esoteric traditions, and the religions of nature of the East. This humanistic expressivism, which is visible in the yoga practiced in the West, is completely different from classic yoga, which we will recall from Eliade's interpretations is not humanistic and is about a complete withdrawal from the evanescent life.

Taking into consideration the afore-mentioned thoughts on practicing yoga in the context of Western culture, it can surely be called a religion of everyday life, which is not always seen as a religion. This means that only those elements from Hindu traditions of yoga were chosen which can be used autonomically and independently by individual in his or her life choices. The subject, independent in its choices from others (in its own belief of course), concentrates on its own well-being and physical and mental health, choosing those spiritual aspects of yoga which suit him/her. These include, of course, physical exercises (asanas), the concept of the individual as a unity (unity of body, mind, and soul), harmony and connection with the universe, development of skills to survive difficult situations, the idea of the transience of the material and mental world, and not blaming others as the blaming is a source of one's suffering. If he/she so desires, then he/she can also accept the concept of God, not interfering in life but as one who can be found inside by corporal practice. All of these elements are important in everyday life and in the functioning of individuals at work and in society. They give them peace in interactions, emotional balance (which is important in relations with others), and develop the individual spiritually and physically, thus, the enabling fulfillment of the compulsion of constant development and acceptance and self-realization that the late modern culture imposes on the individual. The individual develops and chooses his or her individual style of life for the good of others with whom she/he meets in the family and at work, but also with those whom he/she does not meet, since the holistic philosophy asserts the 
connection of all things in the universe. By practicing yoga we become better on an everyday basis, which is used by everyone around the world. This also partially concerns the natural resources of the world. Hence that which is profane in our everyday duties and everyday life can be spiritualized, because when I calm myself and I am emotionally calm, I am emotionally empathic, I am distanced to the material world, I am friendly towards others, I do not judge them, and I am tolerant, or concentrated on one point or meditating on a currently watched object or a currently performed action I experience every day. I do not need an external ritual framework of religious acts, because all acts can have a spiritual character in the here and now. This is what yoga teaches us when it becomes a religion of everyday life for the many practitioners who do not pay so much attention to sacred aspects and the idea of a transcendental God. 


\section{CHAPTER 2}

\section{The social world of yoga practice}

\subsection{What is the social world?}

The social world is some form of association based on mutual interests and the undertaking of various actions by individuals, and this is what defines belonging to this world.

Here, I use the term 'social world' as it was described in interactional sociology by A. Strauss (1978; 1982) and his successors (Becker 1982; Star, Griesemer 1989; Clarke 1991; see also Kacperczyk 2005). The social world centers around some basic actions which it selects. You can distinguish many social worlds, for example, the world of qualitative researchers, the world of practicing yoga, the world of climbers, the world of dance, the worlds of postage stamp collectors, non-conventional medicine, tourist travel, etc. While the world contains spaces where the action can be carried out, technology, especially innovative technology, also which allows one to carve out for a given world both symbolic and physical space. In a social world, we usually encounter some division of work. Some persons deal with justifying the existence of the given social world (legitimization and theorization, as well as public relations), others searching for new spaces for extending its work, others are involved in the development and teaching of technical skills, etc. Some do all of these things on their own. There are also many auxiliary activities, such as defense and attack, creating associations, which help to achieve the main action (Strauss 1978; Kacperczyk 2005). Arenas of disagreement also arise: who is an authentic member of this world, what are its boundaries, what are the justifications for its existence (so-called legitimization), and so forth. Sometimes there is a boundary object around which the disagreement takes place.

The definition of 'belonging' to a particular world is connected with the performance of certain primary activities which, in the case of hatha-yoga, involve performing certain practices. The level of advancement in the practice describes the social identity of the practitioner. Some have a vivid identity and some less so, and many balance on the border of several particular social worlds and accept and juggle many identities, often ending up on the border of all the worlds in which he or she operates. 
One may encounter statements that those who practice yoga are a religious sect, but are yoga practitioners really a religious sect? It seems not. Sects create an alternative vision of the world, connected both with their knowledge and learning spheres as well as social relations. They are considered from the point of view of the wider society somewhat as a closed and deviant communities. Sects often have an exclusive character, not tolerating a double identity, not allowing the use of two sets of rules, and not tolerating encroachments on certain secrets and taboos. The totality of sects creates a high level of conforming behaviors among many participants (see: Wilson 1992: 179). People practicing yoga do not belong to such a closed community. Persons practicing hatha-yoga in the modern world can also be practicing Catholics (or followers of other religions) and take part in Catholic or Protestant church ceremonies and family rituals. They have somewhat of a dual identity. They do not live by the norms of one religion, and are characterized by syncretism in accepting different religious rules.

One yoga teacher, advanced in his practice and a respected authority among his students, had a Catholic wedding. However, during the ceremony he marked his different identity as a yoga practitioner by his clothes. The young couple had wreaths on their heads made of flowers and the groom himself wore a Hindi outfit (data from a participant observation), although the ceremony itself was held according to Catholic rituals. The difference was also marked during the wedding reception. The reception was preceded by a walk by the river. The food during at the wedding reception was from an Indian restaurant. It was vegetarian and there was no alcohol, which is a rare situation in the Polish context.

\subsection{Hatha-yoga practitioners and their social world - arenas and legitimization}

The social world of yoga practice is based on small communities that are sometimes built at or around yoga schools. These communities have their own internal rules. One of them is equality among all practitioners. Outer social hierarchies are put on hold and practitioners are bound by the inner rules of the world of yoga practitioners, which include the rule of equality. The bonds among the practitioners are often very strong and transfer into everyday life outside the yoga school/s, and that which connects the participants of the world together is their passion for hatha-yoga: 
What is interesting, I have noticed that yoga brings people closer. There are different people coming to the meetings, professors and students, young and old ladies. Different, I tell you; and all of them seem to have the same fun, treat each other as equals and everybody is content. There is nowhere in the world where a student could spend time together with a professor or perform some exercises in a pair (laughs). It's something incredible. Maybe you should try.

What is most important from my point of view is that these ties are very strong and often it turns out that people know each other for many years thanks to yoga! They visit each other every day, their families know each other, they go on holidays together, and this is all thanks to meeting at one of the yoga meetings. That is why I think that everything is in this unique, sometimes mystic atmosphere that occurs during yoga meetings, and after the initial astonishment there is simply one big passion and engagement in matters of the meetings themselves, organizing meetings of yogis from all around Poland, mutual meetings in the park during the summer - there are many such [meetings]. Many events, happenings, with some interesting characters showing up at our meetings... (practitioner for 3 years).

Identification with the society is also very important. The interlocutors often refer to themselves in the plural, which also shows positive assets that they possess: "We are better read, more healthy, and more friendly towards others" (practitioner for 3 years).

- And tell me, do you know other people that practice yoga? If yes, then what meaning do they have for you?

- (A moment of thought) Contact with other people who do yoga is very important for me because if we do something on our own, our mind starts more or less doubting it, as if the inspiration in our mind becomes smaller when we do it on our own, as if against the whole world around us. This is why this contact from a psychological point of view is very valuable, as though it is profitable on a deeper level. It is worth doing yoga with other people. Personally, I meditate individually every day, however, once, twice, or three times a week I meet with other people who do yoga and then we meditate, as well as do other yoga practices, in a group. This gives us additional benefits (practitioner for 14 years).

One of the values of the social world is the existence of arenas of disagreement, where different ideologies clash, involving rationalizations connected with the practice of yoga, theoretical justifications, common definitions contrasted with other definitions, and so on.

The quotation below points to the existence of such an arena in the world of yoga. People observing the exercises of David Swenson on YouTube discussed, in comments below the film, the essence and definition of yoga - what is yoga? ${ }^{1}$

${ }^{1}$ See: David Swenson, Ashtanga yoga; http://www.youtube.com/watch?v=xeWmcXbmBY (accessed: 6.02.2011). 


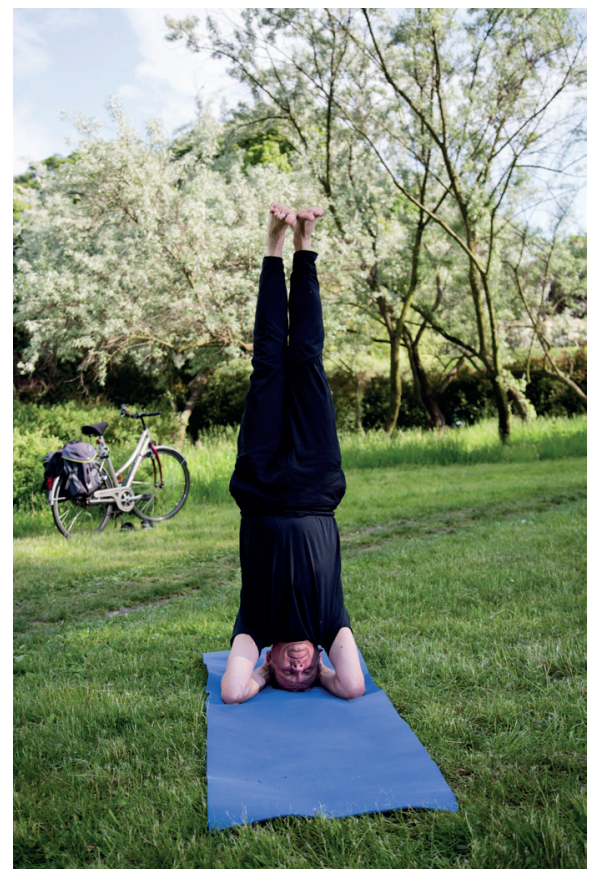

Fig. 2. Author, Krzysztof Konecki, connecting tourist cycling and yoga practice (photo by Kamil Głowacki) (see also David Swenson and his "yoga acrobatics" - ashtanga yoga) ${ }^{2}$

The first opinion mentioned below is the opinion of a person who pays attention only to yoga's physical aspects and the flexibility of the body. The second opinion is very judgmental towards David Swenson himself, as well as the above-mentioned comment. For the second commentator on YouTube, yoga is not a sport but rather a spiritual domain of activity. Yoga has a spiritual path and is not only a domain of physical or social activity. There is then a conflict between two concepts of yoga, physical and spiritual:

i took a workshop from David in march of this year. i am 5'2" and $180+$ lbs... and far from the cheerleader flexibility i had 18 yrs ago! David completely changed my perspective on my practice. he told us that the inflexible people are the lucky ones, because our practice begins the second we stand on our mat... the flexible people can practice for months/years before they are challenged and not until then does their true practice begin. yoga is awesome... go for it!!! (aimeesueaase)

2 http://www.yogashop.nl/davidswensonteachertraining 2014?language =en (accessed: 6.02.2011). 
The second comment is a reaction to David Swenson's film:

That's exactly what's wrong with this video. Yoga is not a sport. It helps you improve life in all aspects and ultimately it is a spiritual discipline. It's goal goes beyond physical and social to the discovering of the true self nature. In conclusion, it's good to do it almost any way (on the condition that you don't cripple yourself by forcing yourself) and best to find a real yoga teacher that is capable of guiding you through the windings of the spiritual path. (crebegea)

Paradoxically, the physicality of yoga helped one practitioner to become incorporated to the world of erotica and advertisement. This aroused obvious criticism. When Sarah Jean Underwood did yoga positions for Playboy in the nude, her action was met with a strong objection from the President of Universal Society of Hinduism, Rajan Zed, who protested against the 'bad usage' of yoga for mercantile reasons: "Yoga is one of the six systems of orthodox Hindu philosophy and it is highly revered in Hinduism. It is a serious mental and physical discipline by means of which the human soul can unite with the universal soul." However, despite the criticism, Playboy concluded that more people were excited than upset with the film and thus the video was not removed from its website. ${ }^{3}$ Emblems of yoga are also used in pornographic films (this refers to both yoga positions and using the name of yoga in the titles of pornographic films).

This extract from an interview with Sławomir Bubicz also shows the existence of other arenas of disagreement:

I met Gabriella (Gubillaro) in Pune at Iyengar's, she had a good practice. However, at that time she did not consider herself to be Iyengar's student, but Dona Holleman's. Then, there was some misunderstanding between Iyengar and Dona. Gabriella, of course, took Dona's side. They had their vision of how yoga should be taught. In the book, Gabriella wanted to show asanas as she understood them. She had the idea to show the work in asanas by using arrows in the pictures, which was not used in Iyengar's books (Moćko 2007).

Here I will focus on the phrase "She had a good practice." Such language forms allow us to categorize and name the participants of the world and at the same time distinguish that the core of this world is those yoga teachers who have a good practice. If yoga has arenas, then there are also subworlds of yoga. Any kind of yoga can be treated as a subworld of yoga: the subworld of hatha-yoga; the subworld of ashtanga yoga; or the subworld of kundalini yoga (kundalini yoga is

${ }^{3}$ See: http://www.allvoices.com/contributed-news/7129343-hindu-outrage-at-playboynaked-yoga-video (accessed: 16.10.2011). 
something different than what is considered hatha-yoga and ashtanga-yoga) (Jung 2003), and so forth. Certain subworlds create similar internal behaviors and similar identities. Some of the participants have the notion that there are differences in the world of yoga:

And, I think that in the world this is yoga culture. People who train have the knowledge of what yoga is, that is, I think they are in some sense similar. And those who do ashtanga are all crazy and very similar to each other. (yoga teacher, practitioner for 12 years, ashtanga yoga)

Arenas of disagreement are also connected with the problem of certification in teaching yoga. Legitimization of the social world can be gained by the granting of certificates. Certificates were also introduced by Iyengar, who wanted to unify his system of teaching and prevent significant confusion in the methods used. Certificates also show the line of teaching and where the students come from (Iyengar 2002b: 164). Certificates in Poland are issued by Iyengar's Institute based on its exams, but also by the Association of Spreading Physical Culture. The latter entity is supported by yoga experts working in officially recognized institutions, such as universities where yoga is taught as a rehabilitation and physiotherapy method. Such certification is supported by Professor Lesław Kulmatycki, a yoga specialist. He criticizes the situation whereby many yoga schools are run by teachers who are not qualified, without valid certificates, and that the commercialization of the practice has gone too far and yoga is now present in fitness centers. He criticizes the so-called 'instant yoga', a sort of gymnastics where quick effects are promised without including the spiritual side of yoga in the yoga practices (Augustyn 2009). Lesław Kulmatycki and Zdzisław Burzyński claim that: "A positive phenomenon is the quantitative development and self-organization of the teachers. However, the lack of criteria and its fortuitousness when it comes to education and special courses or training which allow for running classes can be considered disturbing." (2008: 170). The lack of information in websites about the master and the tradition he comes from awakens the above-described fear, and the problem of authenticity and credibility of yoga practice arises:

Such information makes the teaching itself trustworthy and states not only the source way (in comparison to book one) of acquiring the art of yoga, but is also information for those looking for the right, in the cognitive and recreational sense, class or school (Kulmatycki, Burzyński 2008: 171).

In my analysis of the Internet pages of 172 yoga schools, only $27.9 \%$ of the schools had a certificate. 
Legitimization is a very vital process in the social world. It is also a characteristic justification of rightful existence in this world. This applies especially to yoga teachers, who in their advertisements underline their education, experience, and certification in the world of yoga and contact with important teachers. Here is a quotation from a webpage describing one of the hatha-yoga teachers:

Experience:

- student of Konrad Kocot,

- has a national diploma as a Hatha-Yoga instructor issued by MENiS (Ministry of Education and Sport),

- member of a Polish Association of Iyengar's Yoga,

- graduate of training and workshops organized by Konrad Kocot, Geetan Iyengar, Gabriella Gubilaro, Lois Steinberg, Corin Biryan, and Yoga Master Ajit Vardan Singh, with whom she organized workshops in Lodz, Poland in 2007,

- organizer of Hatha-Yoga workshops and training for coaching companies,

- graduate of Law and Administration Department at the University of Lodz. ${ }^{4}$

Among the 172 yoga schools' websites which I analyzed, 77 (44.8\%) refer to yoga masters and yoga gurus from abroad, and 48 (27.9\%) refer to masters and yoga gurus from Poland. Here is an example of such a practice of legitimizing one's own business:

...practicing yoga according to the teaching of B. K. S. Iyengar from 1993. Has an Intermediate Junior II degree. His first steps were done with Sławomir Bubicz, precursor of B. K. S. Iyengar's yoga in Poland. In January 1996, after being made and allowed by his teacher, he started his adventure with yoga teaching. From that moment he has been constantly deepening his knowledge and practice in numerous courses and workshops conducted by famous teachers from all around the world.

He was in the Ramamani Iyengar Memorial Yoga Institute in Pune many times, where in 1999 he received his first diploma. He practiced and assisted in therapeutical classes under the watchful eye of Guruji and his children - daughter Geeta and son Prashant. ${ }^{5}$

Showing the history and traditions of a given school are an important part of legitimizing the work of the school and the teachers working there. Among the 172 yoga schools' websites researched, 42 (24.4\%) describe the history of the school. Showing the source of the yoga practice is also very important, and among the 172 above-mentioned yoga schools, 33 (19.2\%) describe the culture of India.

${ }^{4}$ See: http://www.yoga.net.pl/category/index/site/yoginki-centrum-yogi (accessed: 24.02.2011).

${ }^{5}$ See: http://www.hathayoga.lodz.pl/nauczyciele.php (accessed: 31.07.2011). 
The world of yoga is also legitimized by book publications about yoga and training offered in its practice. There are many such publications in Poland. There is also a publishing house (Virya, established in 1995 by Sławomir Bubicz) which spreads not only the philosophy of hatha-yoga and yoga in general, but also offers text books explaining how to perform asanas. Bubicz decided to spread yoga through translating and publishing books by Iyengar, including books describing the philosophy of hatha-yoga, not only its physical side (Moćko 2007).

In the social world of yoga the practitioners, and especially advanced practitioners, pay attention to the authenticity of the practice. This concerns whether the practice agrees with the rules and knowledge we have about the ideal practice:

And later I came here to Łódź, and there was ashtanga, but it was not a real ashtanga, so I started with the real ashtanga, started teaching here and took a very seriously approach, that if it is called ashtanga then I will teach ashtanga and this will be a real ashtanga. (practitioner for 12 years)

The world of yoga has its own hierarchy. Positions in it are marked by the advancement in practice, which is judged by experienced teachers (gurus). This world undergoes some institutionalization, and as has already been mentioned there are certain 'recognized' workshops and certifications of skills are issued. Participation in workshops is also emphasized in interviews.

Then, I went to this teachers' course training in ashtanga with $\mathrm{X}$, and it was 10 days, 50 hours of very intense learning how to be a teacher, I'd already engaged in a few things like this and it was not the first time, but only the first time with ashtanga. And from then on I can really tell myself I am an ashtanga teacher. Not fully, because I have not been to India yet and learned and studied ashtanga in Mysore with guru Sri Pattabhi Jois. My plans are to go to India and practice in Mysore, for sure for a month or two months or something like that. I hope that this will happen because in this system of yoga, in ashtanga, it is not like that you go to somebody and they give you a certificate that you can be a teacher - you have to go to India, you have to go there three, four times for like two, three months. And a guru has to look at you when he decides how you practice. He will see your character and will let you either teach, or will not allow you to do so, and that is it. (yoga teacher, practitioner for 12 years, ashtanga yoga)

In the world of yoga practice, there is also a common theorization, an explanation of yoga philosophy and its Eastern heritage. The concentration on the present and the importance of the road itself, not achieving the goal, are emphasized. The author of the statement below also does a meta-reflexion, asking herself: did her thoughts already 
exist in her before she started her practice, or are they an effect of her practice? This is an important question to every theoretician, albeit less important for the yoga practitioner:

That you cannot have all like, er, in one moment. And that... and that the road counts more than the aim, right? And this is also an important particle of the thinking, that it is this road and not the aim that is it. For example, this thinking had a very big influence in my life, that, er, and I do not know if it was more because I was going to yoga or I had this thought in me and yoga only confirmed my thinking that the process is the most important because the process is always the present, right? The aim is always in the future, and it's the present that's important, the process is important. (practitioner for 5 years)

Theorization also applies to the explanation of the attitude toward the body present in our culture in comparison to the attitude in the Eastern culture, where yoga comes from. Corporal dimensions turn out to be as important as the spiritual dimension.

My body and that corporal particle was totally suppressed, right? It did not exist for me; it was always something worse, something not important, right? Uh... also, yoga and this whole Eastern system teaches something like... that the body is as important as the mind and that one penetrates the other, that in truth these are the same, uh, it is this, these are two different aspects of the same thing and that also this body is the temple of the soul, right? And you integrate the body with the mind. (advanced practitioner, no data on the length of practice)

In the theorization by the interlocutors, one also finds analysis, diagnosis, and assessment of Western, Christian culture. We are used to material comforts and matter itself (empty attachments to matter). We do not perceive the importance of the corporal particle in our life, even though we belong to the cult of the body (the empty body cult).

Because we live too fast, we smoke cigarettes, we drink coffee (laughter), we take many painkillers making our body work at such speeds as we desire, we sleep during the day, we work at night, right? It is somewhere mixed against this nature and it is in this direction that, er, the body is put in the first place in a completely shallow way, right? That is, corporality is such an, er, well, such an empty something, such attachment to the matter, such an empty attachment to the matter, that we believe that this matter is, I don't know, er, eternal, that this youth is eternal, that this beauty will last forever and, er, in the body cult, in such an empty body cult as we live in, at the same time it's suppressed, like the corporal particle like in the second case in which we do not think at all, for example, we do not think about it but live somewhere only or only with such a spirituality disconnected from it. For example Christianity, in my opinion at least, is like that; it completely omits this corporal particle. I mean, not Christianity itself, but in such form as we have, right? Because I cannot say this if I'm not so deep in it. But, somewhere its there, right? We dispraise the body, dispraise the matter, earthliness, right? And for example, in the East, there 
is a completely different attitude to it, right? This body is the temple of the soul, and this is unity, that there is a feeling of unity we do not have here and maybe this is why this is so attractive for people here in Europe, because somewhere, er, on the way to develop this culture there was a split, it was not always there, right? (advanced practitioner, no data about the length of practice) ${ }^{6}$

\subsection{The intersection of the worlds}

\subsubsection{Intersection of the world of yoga practice and the world of climbing}

Some people engaged in high-mountain climbing use yoga practice in order to improve their results in their basic performance (e.g., in the sport currently practiced). They search for a yoga which concentrates on their inner balance; the balance with nature. Yoga intensifies the climbing experience. That is why they use yoga's physical and breathing exercises, as well as those connected with energy flow. Climbing is thus the embodiment of animalistic parts of nature. However, yoga helps them get outside their terrain. Some of them are shocked that with all their physical endurance they sometimes feel like beginners during their yoga classes. ${ }^{7}$

Chris Sharma, a professional rock climber, believes that sometimes it is good to be a beginner and get some advice from yoga teachers. Anything that helps to get to the top is good, according to him. He wants, as he himself explains, to open his kundalini (possibly he means energy channels). In the quoted film he is shown also doing asanas (e.g., Crow asana, which he was unable to perform, Warrior III asana), and exercises such as yoga with a partner, and he is also shown climbing rocks. In the film one can vividly see the attempt to visualize the intersection of these two worlds; the pictures of yoga exercises crisscross with pictures of rock climbing. Yoga is used here instrumentally in order to improve the climb.

\subsubsection{Intersection of the world of conventional medicine and alternative medicine}

Conventional medicine has begun treating yoga as a part of alternative medicine, or non-conventional therapy, a term possibly connoting more respect. Official research has been done on the influence of

\footnotetext{
${ }^{6}$ For more on the subject of commonsense theorization, see Chapter 3.

7 See: Chris Sharma's statement in the film Chris Sharma Does Wanderlust, http:// vimeo.com/14313670 (accessed: 14.08.2012).
} 
yoga on human health. This is related to the fact that patients spend more money on alternative medicine, thus official institutions have to deal with this problem since it applies to the health of the whole society. This is the case at least in the U.S.A. (Augustyn 2010), where it is often explained and shown in a scientific and modern way how the organism works (specific parts of muscles and sinews) during asana exercises. Hence science is being connected with traditional yoga, in order for one to give the other more credibility. ${ }^{8}$

There is also research done about the positive effects of yoga on the human organism and its physical and mental health. Publications appear in academic magazines connected with alternative medicine, like The Journal of Alternative And Complementary Medicine.

It has been proven experimentally that doing yoga improves the mood and lessens anxiety among practitioners much better than in the case of persons who take a stroll. Yoga has also been used to reduce the symptoms of depression, anxiety, and epilepsy (Streeter et al. 2010: 1145).

Something like the medicalization of classic yoga is taking place (De Michelis 2008: 25). Here the psychosomatic elements of yoga are accented, which is in accordance with the modern trend to look for therapeutic elements in different practices and philosophies of the East. Yoga performed for mental and physical health has been more or less accepted by the world of conventional medicine. Yoga is very often helpful in post-accident rehabilitation. Many people have taken advantage of such help and some of them have even travelled to Iyengar's Yoga Institute in Pune, India (data from participant observation). Popular publications also help with the medicalization of yoga by showing its positive effects on health (Javalgekar 2008; Reignier 2010). What's more, many celebrities and famous people have helped popularize yoga. Yehudi Menuhin wrote the introduction to Iyengar's Illustrated Light on Yoga, and in its Polish version Jacek Santorski, a psychotherapist, also underlines the health effects of yoga practice, as follows:

I witnessed physical miracles and emotional healings of persons practicing yoga according to Iyengar. Every time it was a miracle resulting from the hard work of a student and the teacher. This yoga is a system of healing and cultivating the body - the temple where the spirit lives. It happens without any mystification connected with magic or extra-terrestrial rituals (Iyengar n.d.: 8 [Introduction to Polish edition]).

\footnotetext{
${ }^{8}$ See: http://www.bandhayoga.com/keys_hams.html (accessed: 19.05.2012).
} 
Gurus are also visible in the process of medicalizing yoga. They accent the medical elements in their work and also in the practical exercises. Iyengar many times underscored the healing effects which come from yoga practice. In his books describing asanas, one finds descriptions of healing effects. Below I quote an example dealing with the asana called Sarvangasa (in Polish it can be called świeca [the candle], similarly as the English candle pose, and in German Kerzenstellung).

The importance of Sarvangasana cannot be overemphasized. It is one of the greatest boons conferred on humanity by our ancient sages. Sarvangasana is the mother of asanas. As a mother strives for harmony and happiness in the home, so this asana strives for the harmony and happiness of the human system... Due to the soothing effect of the pose on the nerves, those suffering from hypertension, irritation, shortness of temper, nervous breakdown, and insomnia are relieved... The asana is recommended for urinary disorders and uterine displacement, menstrual troubles, piles, and hernia. It also helps to relieve epilepsy, low vitality, and anemia. It is not an overstatement to say that if a person regularly practices Sarvangasana, he will feel new vigor and strength, and will be happy and confident. (Iyengar 2005a: 94)

Thus apart from the health effects connected with the body, we also have mental effects; we gain self-assurance, become relaxed and liquidate negative emotions and stress.

\subsubsection{Intersection of the world of business corporations and the world of yoga practice}

Yoga, and especially asana practice, has become a very popular activity in the Western world. Yoga focused on performing positions and physical exercises also became a very big business. Styles and hatha-yoga positions have become legally protected properties and are sometimes franchised. Yoga equipment is sold and yoga positions are used to advertise mobile phones, yoghurt, and other products. In 2008, according to Singleton, in the U.S.A. alone yoga practitioners spent 5.7 billion dollars on yoga classes, holidays with yoga, and products connected with the practice, which is more or less half of Nepalese gross national product (2010a: 3). Doing yoga requires some expenditures in the U.S.A., where yoga is practiced by middle-class people with higher education, mainly with an annual income over 75000 dollars. In Poland, businesses connected with hatha-yoga are also highly developed, from hatha-yoga schools to publishing houses to the production and sales of different accessories for yoga. 
According to specialists, yoga reduces stress. Thus it can be helpful in corporations because it helps to improve concentration and raise the self-confidence of employees. All of this helps to build an appropriate attitude toward work and is pro-effective because it limits operational costs. Pro-health programs reduce the costs of medical treatment incurred by the companies, and it is estimated every dollar invested reduces costs from 3 to 6 dollars. Many businesses promoting yoga advertise themselves and offer their services directly to companies.

Some pictures visualize the intersection of the world of industrial corporations (conference desks, chairs, and glass-walled offices) and the world of yoga (a person doing yoga sitting on a conference desk in an easy pose, sukhasana, with hands placed together in a praying tone). Two different symbols are thus juxtaposed in one place and time. ${ }^{9}$

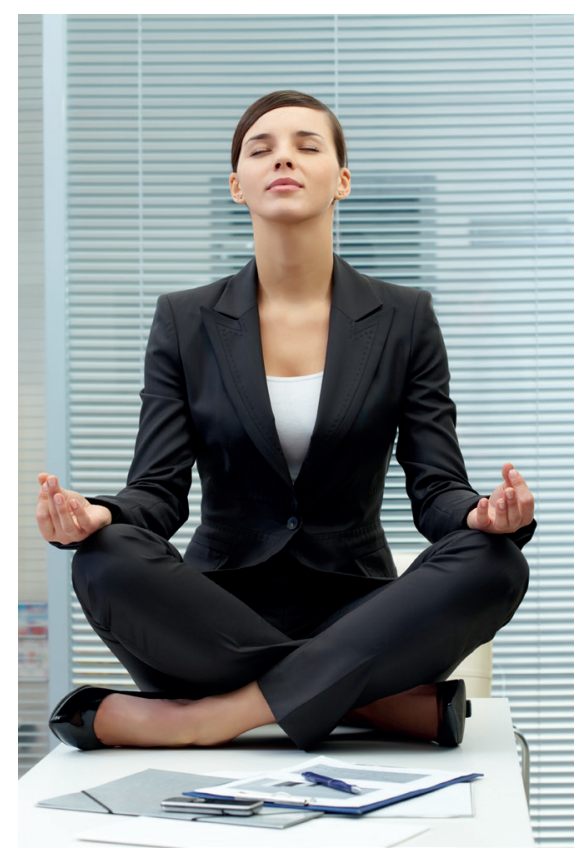

Fig. 3. Photo showing the connection of yoga meditation and work in a business organization $^{10}$

${ }^{9}$ See: http://www.questyoga.com/classes/corporate-classes (accessed: 5.01.2011).

${ }^{10} \mathrm{http} / / /$ pl.depositphotos.com/11629665/stock-photo-meditating-on-table.html. 
Below is an advertisement of one so-called 'business yoga' company in New York:

Business Yoga is a contribution to the quality of life, health, and productivity of your employees. It provides an immediate return on investment through profound stress reduction, improved feelings of health and well-being, and higher mental energy and strength.

Suitable for a meeting or conference room, Business Yoga exercises are performed in work attire while employees sit in chairs. No previous yoga experience is required, so everyone can participate. The instructor leads participating employees through exercises that address the challenges of today's business environment and break through negative thinking.

Experience the ultimate benefits of the Business Yoga wellness program:

- Fewer sick days taken

- Reduced daily conflict/aggression

- Improved productivity from higher mental and physical stamina

- Better communication and team cooperation. ${ }^{11}$

This is one example of what we can call the 'commodification of yoga'. Yoga schools and yoga centers where you have to pay to practice yoga demonstrate that yoga ideas are for sale. What's more, there are patents for certain yoga exercises, trademarks, and legal rights connected with different yoga products (De Michelis 2008: 24-25). Original yoga ideas are being redefined in the modern world of business and large organizations. It is a kind of recycling, a rebranding of old ideas using new packaging. However, we are not always aware of the mechanisms that govern the meanings in modern organizations (Magala 2009: 3-6).

Yoga schools are often strictly business undertakings. Classes where one has to pay to enter are organized. Prices are often calculated in such a way that you receive discounts for multiple entries. Yoga schools advertise in newspapers and on the Internet. Of the analyzed 172 yoga schools' websites in Poland, 131 (76.2\%) give their price list. It is thus an economic activity. Yoga schools often organize field trips in different more or less luxurious resorts. Yoga is combined with other activities, such as sunbathing, learning about healthy eating habits, body detoxification, and even yoga on surfboards. ${ }^{12}$ Field classes, really field trips, are sometimes held abroad,

11 See: http://www.businessyoga.us/Yoga-for-companies--Yoga-for-healthy-employeesand-managers (accessed: 6.02.2012).

${ }^{12}$ See: http://wiadomosci.onet.pl/fotoreportaze/ciekawostki/joga-na-desce-serfingowejjogaqua,5013638,0,fotoreportaz-maly.html (accessed: 2.02.2012). 
for example, in Thailand. ${ }^{13}$ In the analyzed websites of Polish yoga schools, $46.2 \%$ of the schools offer the possibility of participating in field classes.

\subsubsection{Intersection of the world of yoga and pop-culture}

Yoga practice has also become a popular topic in media (in Poland one can find articles in the leading newspapers and weeklies, like Gazeta Wyborcza, Rzeczpospolita, Wprost, and Polityka). Yoga is connected with entertainment, leisure time, and relaxation. Yoga is done by many celebrities who are popular in mass media.

Every year events are organized in many cities. For example, Yoga in the Park attracts many people curious about new experiences, usually taking part in many different activities, such as learning Jewish dances, promotion of sugar substitutes, classes on creative cooking, classes on food therapy, lectures on healthy life styles, and yoga classes connected with massages. Yoga in the Park is a typical promotional activity for business yoga. During this activity the positive influence of yoga on health and mentality is underscored, and it is said that yoga helps to overcome barriers and mental and physical constraints. ${ }^{14}$ In yoga schools, you can come in contact with other offers of a combined spiritual and therapeutic nature, such as the concert of Tibetan pots and gongs or all-day-long meditations with Hindi masters.

Yoga is also combined with aerobics and music. One such event is the Wanderlust Yoga and Music Festival in California (Squaw Valley). The goal of this event is, according to the organizers, propagation of yoga and its introduction to the masses. It is believed that this is a special place for practitioners full of artistic inspiration, different artists, thinkers, musicians, and rock bands. Many parties are held, where people have fun and dance. Listening to music and dancing joins people together. ${ }^{15}$ The visualization of this intersection of these worlds is visible in the above-cited video clip, where one can see participants dancing at the festival, musicians, and also practice sessions of hatha-yoga. The pictures intertwine together in a syncretic patchwork, symbolizing the inclusive character of the festival; a great ritual for which yoga is the basic action, assisted by other actions, originally not connected with it, such as listening to rock music.

13 See: http://www.yogabodynaturals.com/new-year-retreat2012?awt_l=O0Lpg\& awt_m=Jk76dlBWQE4rf9 (accessed: 2.08.2011).

${ }^{14}$ See film Yoga on Grass in Zrodliska Park in Lodz, http://www.youtube.com/ watch?v=0FXnairU258 (accessed: 13.08.2011).

15 See: http://www.vimeo.com/14487629 (accessed: 1.08.2011). 
During the festival, yoga schools, owners of schools, and festival sponsors advertise, as for example the company Prana. ${ }^{16}$ This shows that in this ritual at least three worlds intersect with each other: the world of hatha-yoga, pop-culture, and business.

\subsection{A disagreement about hatha-yoga origin. The vision of Marc Singleton}

There is disagreement in the world of hatha-yoga about the origin of modern hatha-yoga. Some believe it goes as far back as the roots of ancient India, others that it is a creation of the modern world. This conflict joins with the earlier-mentioned disagreement about whether authentic yoga is a spiritual practice or a physical one. ${ }^{17}$

The current interest in yoga in the Western world was surely one of the motivations for Marc Singleton to write his book (2010a) about the origin of hatha-yoga. The book is very important as it breaks some stereotypes connected with the origin of the modern hatha-yoga practiced in the Western world. It is a wonderful historical analysis of the development of modern hatha-yoga, the one we know in the West from the ever-more-popular hatha-yoga schools, advertisements, and the images of many celebrities. ${ }^{18}$

It turns out that the Western world had, in the past, a similarly strong influence on creating the hatha-yoga practiced in India as on the modern hatha-yoga. Modern hatha-yoga is also our Western, modern invention. Marc Singleton is mainly interested in how it happened that yoga, in its current shape as practiced in the Western world, does not resemble its state from the $19^{\text {th }}$ century in the country (at that time a British colony) from where it originates. He is interested in the pe-

16 See: http://www.vimeo.com/15470104 (accessed: 1.02.2011).

17 On YouTube there is a film from 1938 where B. K. S. Iyengar shows a complicated, somewhat acrobatic, vinyasa based on elaborate physical body flexibility - see: B. K. S. Iyengar 1938 newsreel Part 1 (SILENT); http://www.youtube.com/watch?feature $=f v w p \& v=l m O U Z Q i 6 T w \& N R=1$ (accessed: 17.04.2012). Appended to the film is a comment underneath defending the belief that the roots of yoga come from ancient India: [speedracer77] "Yoga dates back over 5000 years to Vedic times. You cannot say something is traditional Yoga simply because it dates back to 1938. In the most literal sense, a master acrobat (i.e., from a circus) could perform these moves and have nothing to do with Yoga. The origin of Yoga is a spiritual discipline aiming to achieve self-realization, stillness of the mind, and transcendence of attachment and ego. The video shown is one leaf on a branch towards those roots but is not the root itself."

${ }^{18}$ In the description of this book, I mainly use the review of the book at hand (Konecki 2011a). 
riod between 1896 (the year of publishing Swami Vivekananda's book Raja Yoga [criticizing yoga concentrated on asanas, see: 2003]) and 1966 (the year of publication of B. K. S. Iyengar's Illustrated Light on Yoga, which shows asanas and the rules of their practice). This was the most creative time in the development of the practice of hatha-yoga in the way we know it today in the Western world. Yoga experienced a revival in India in the second half of the $19^{\text {th }}$ century thanks to Vivekananda (1863-1902). But, even then asanas (postures in yoga, the physical side of yoga practice) the way we know them now did not appear in Vivekananda's system. For him yoga was about gaining spiritual knowledge, knowledge about oneself, and applying it to everyday life (Vivekananda 1982). In general, the physical side of yoga was disregarded by Vivekananda and others who followed him. So, where does the modern yoga (anglophone: transnational yoga) practiced in the Western world come from? [Singleton 2010a: 40]).

Singleton puts forward a thesis quite provocative and controversial for many readers. He claims that in the West in the $19^{\text {th }}$ century there was an international movement of physical culture that influenced Hindi youth at the turn of the $19^{\text {th }}$ and $20^{\text {th }}$ centuries. Europe also developed quasi-religious forms of physical culture that influenced the interpretation of yoga. The nationalistic Hinduism that began developing during that time also influenced the understanding of hatha-yoga as a traditional form of physio-spiritual practice from India. This is also seen in the more modern texts of one of hatha-yoga gurus, Sri Pattabhi Jois:

Were the government itself to understand their usefulness and make the practice of yogasana, the Surya Namaskara, and their tradition compulsory for all students in all educational institutions, boys and girls alike, which would help to render their lives pure, it would be doing a great service to the world. Indeed Mother India would be very pleased. We should, therefore, never forget to carry the torch of this divine light of yogic knowledge, which has been passed down to us with our Vedic culture, and to keep its flame alight for all eternity (Pattabhi 2010: 43).

Thus there was developed in India a Western physical culture enriched by asana practice, which returned again to the West and connected with the Western, "esoteric gymnastics" that developed in Europe and the U.S.A., without earlier contact with yoga tradition.

Posture-based yoga, as we know it today, is the result of a dialogical exchange between para-religious, modern body culture techniques developed in the West and the various discourses of "modern" Hindu yoga that emerged from the time of Vivekananda onward. Although it routinely appeals to the tradition of Indian hatha-yoga, contemporary posture-based yoga cannot really be considered as a direct successor of this tradition (Singleton 2010a: 5). 
In the resources of the Oxford Library and in the Hindi Department of the British Library, the topic of asanas and hatha-yoga did not appear in hatha-yoga textbooks. The same conclusions resulted from viewing the American literature until the year 1930. Only after World War II did popular English yoga textbooks begin to strongly emphasize the position of yoga, which did not happen earlier (Singleton 2010a: 5-6). Asanas in English textbooks were compared to gymnastics. Known patterns of body movement interpretations allowed for the incorporation of yoga into Western physical culture. The philosophical frames of yoga were suppressed in the modern discourse of health and fitness. Analysis of the gymnastics textbooks allow us to assume that the English-speaking authors of yoga text books wrote in the modern heritage of physical culture, according to the orthodoxy of hatha-yoga practice, and discarded those parts that were difficult to combine with the evolving discourse of health and fitness (ibid.: 7). One can agree with this thesis even in relation to B. K. S. Iynegar's textbooks, which rather avoided yoga relating to cleansing the organism (satkarma) and traditional ascetic yoga practices, tapas, where the notion of asceticism in the classical understanding of this word is not used at all (see: Iyengar n.d.: 23-24).

According to Singleton, Scandinavian gymnastics derived from P. H. Ling, E. Sandow's bodybuilding teachings, and the methods of teaching physical culture by the YMCA (also in India) had a special influence on hatha-yoga. It also needs to be mentioned that Pehr Ling took lessons in martial arts and Chinese massage from his friend "Ming." It is visible that the world of multinational physical culture is mixed with other traditions of both physical culture and philosophical traditions. The intersection of the world of Hindi yoga and the world of Western physical culture is clearly shown in by Singleton's analysis. Arguments are still going on about the different plots and sub-plots of these mixed traditions. This contentious arena is reflected in the topic of interpretation of the heritage by one of the main and early gurus of modern hatha-yoga, T. Krishnamacharya (1888-1989). The controversy concerns who represents the "clean" system of yoga practice (orthopraxy). There were three most important students of Krishnamacharya who took their classes in the Palace in Mysore: Sri Pattabhi Jois, B. N. S. Iyengar, and T. R. S. Sharma. Another student who became famous in the Western world - and the most popular propagator of hatha-yoga in Poland - was B. K. S. Iyengar (Singleton 2010a: 9; see also the Polish edition of Illustrated Light on Yoga n.d.). Yoga, according to Singleton, is an anglophone cultural phenomenon created as a result of dialogue between India and the Western world through the use of 
the English language, which is why this phenomenon is called "transnational anglophone yoga" (Singleton 2010a: 10).

These mutual influences and the intersection of traditions can be vividly seen in the interpretation of posture/asana called salamba sarvangasana. This posture was known separately in Europe, even though it also existed in yoga together with its original name in Sanskrit:

To take one instance: A shape very much like the shoulder stand (sarvāngāsana) was the emblem of the British Women's League of Health and Beauty during the 1920s. It was not associated with yoga, but rather had its own characteristic set of meanings. It helped one to stay young, trimmed fat around the waist, and so on. A similarly shaped posture is also to be found in medieval Indian hathayoga, where it is called "a secret in all the tantras"... What is important here is not whether the Women's League posture is old, nor even whether it is derived from pre-modern Indian yoga per se, but the accretions of cultural meaning which make it distinct from the posture in the Indian hatha-yoga systems. In probability, the "modern," cosmetically-oriented posture was inspired by Indian yoga traditions. But it is no longer the same posture as that outlined in hatha-yoga texts (Singleton 2013: 54).

The phenomenon of yoga described by Singleton should not be analyzed from the point of view of trying to perceive the closeness to or remoteness from the so-called 'original roots of yoga'. Searching in the classic books of Yajur or $\mathrm{Rg}$ Vedas for gymnastic postures of hatha-yoga is not justified from either a historical or philological point of view.

Sri Pattabhi Jois, in describing the set of asanas called surya namskara, is looking for their roots in ancient India (2010: 34). "The practice of yoga is not new to the people of India. It is a noble, desireless action, coupled with righteousness, which has been passed down, in an unbroken tradition, since time immemorial" (Pattabhi 2010: 3). By following the precept of the great sages:

Shariramadyam khalu dharma Sadhanam [the first duty is to take care of the body, which is the means to the pursuit of spiritual life], our ancestors found the means to bodily health. Such means, they knew, should not violate the scriptures, but be in accordance with them. And this, they knew, could only be possible through the Surya Namaskara and limbs of yoga. So they studied yogic science, brought it into practice, and were joyful. In all corners of India, too, people of every class were bringing the Surya Namaskara described in the yoga shastra into practice [in an ancient Hindi book - K. T. K.] (Pattabhi 2010: 34).

For Pattabhi Jois, the cycle of asanas known as "the sun salutation" is a kind of prayer and has almost a religious meaning. In his interpretation of hatha-yoga, we deal with an apologetic past of ancient India: 
Our ancestors certainly studied the mantras, understood their meanings, and put them into practice. As a result, they lived long lives of good health, great strength, and high intellect, and, without ever succumbing to disease, death, or poverty, they attained divine knowledge, merged with bliss, and were forever content (Pattabhi 2010: 41).

However, from a philological and historical point of view, it is difficult to find evidence for the description of this set of postures in some classic texts. Modern yoga should be, according to Singleton, analyzed rather in its own terms than by its similarity to some traditional Hindi practice (2010a: 14). The yoga that comes from India went through many transformations in the Western world; this was also true in India itself in the past 150 years because of the influence of the Western world and modern philosophy. The attempts to root modern yoga in the Hindi tradition is also visible in B. K. S. Iyengar's book, Light of the Yoga Sutras of Patanjali, where he translates Patanjali's sutras from Sanskrit and interprets them. Here is a fragment of an interpretation: "Usually, the mind is closer to the body and the organs of action and perception the soul. As asanas are refined, they automatically become meditative as the intelligence is made to penetrate towards the core of being" (Iyengar 2002a: 158). Sutra "sthira sukham asanam" was translated as "Asana is a perfect firmness of body, steadiness of intelligence, and benevolence of spirit" (ibid.: 157). After that follows a comment more than a page long. In this comment, he underscores the importance of dharana (single-pointed concentration) and dhyana (meditation) in the correct performance of asana. Iyengar also refers here to sutras III. 1-2 where there is no mention, however, of asana, but only dharana and dhyana. In general, Iyengar is trying to valorize asanas in his comments to Patanjali's sutras. He is also underlining the value of yoga practice, although from the direct reading of sutras one can have also a different feeling in relation to the meaning of asanas in yoga according to Patanjali. Iyengar's great credit is that in his last works he showed to the Western world that yoga is not only asanas, but also a wider system of practice and "experimental" studies of an individual.

In the world of hatha-yoga, there is also an arena of controversy connected with the dispute about the origin of postures practiced in modern yoga. Singleton refers to The Yoga Tradition of the Mysore Palace by Norman Sjoman (1996: 35), in which the author suggests that the "godfather" of modern hatha-yoga, T. Krishnamacharya, developed his system of asanas based on the gymnastic traditions in the Mysore Palace where he lived and taught. He compared the yoga postures taught by the students of Krishnamacharya, B. K. S. Iyengar, and Sri 
Pattabhi Jois, with the ones contained in the exercise textbook found in the palace, and it turned out that they are similar. Sjoman's work was either ignored or met with severe criticism by the followers in search of asanas' roots in ancient India, as it ruined their system of assumptions about the origin of modern hatha-yoga.

Why is so much attention focused on the current practice of asanas? Well, because in the 1950s practical textbooks were published which constituted a modern, secularized, and medicated version of yoga, which was also connected with sport and physical exercises. The 1960s in the U.S.A., together with the youth revolution, popularized Hindi philosophies and yoga as well. There were even shows on TV such as "Yoga for Health." Bringing yoga closer to the New Age movement also had a great significance on its popularization. It constituted a renewed discovery of the relations between yoga and $19^{\text {th }}$ century esotericism. In the 1990s, yoga became a commercial undertaking in many Western countries and cities, and one may now say that its commodification and commercialization is firmly in place.

The search for asana postures in ancient texts or images also evokes controversy. It cannot be proven that the postures that we know today were practiced in ancient India. Maybe in reading the ancient texts and examining the images we are reading our modern perspective of yoga practice into the ancient texts.

An analysis of the $19^{\text {th }}$ century works of Hindi practitioners concerning yoga is of great importance. In general, yoga based on asana practice was discarded and criticized by them. This is why during this time, in the anglophone books about yoga no descriptions of asana practice are present. Yogis in $19^{\text {th }}$ century were shown more as masters of acrobatics and vaudeville antics rather than Hindi spiritual practitioners. The art of acrobatics, which asanas resembled to Western viewers, could be accepted in the West as there was a tradition of acrobatics connected with the flexibility of the body represented by Hindi yogis. The topos of acrobatics as an entertainment was rooted in the Western consciousness.

Some of the postures shown in photographs by Thomas Dwight in Scribner Magazine (April 1889) closely resemble asanas shown by Iyengar in his book Illustrated Light on Yoga (see: Singleton 2010a: 58-64). It needs to be mentioned here that the popular image of yoga and yogis in the $19^{\text {th }}$ century and in the beginning of $20^{\text {th }}$ century was not very positive. They were often shown as drifting, dirty, uneducated, and begging ascetics, and they were similarly seen by the Hindi elite back then (see: ibid.: 69). This shed a negative light on the perception of the performance of asana practice. 
Critical attitudes towards hatha-yoga were seen in Raja Yoga (1886) published by Swami Vivekananda. According to him, hatha-yoga practice (asanas) is very difficult and cannot be learnt quickly, and it does not lead to spiritual development (ibid.: 71). For Vivekananda, Raja yoga is the real yoga, and the simple physical exercises of hatha-yoga are not real yoga. For him "knowledge is power." Singleton claims that this assumption comes from the rhetoric of American New Thought (ibid.: 71). Christian Science New Thought was established in New England in 1880 as a para-protestant movement that spread the notion of inborn divinity of self and the strength of positive thinking, updating divinity in the world and supposed to lead, as a consequence, to riches and health of an individual. It was thus believed that yoga was a long-term and exotic repository of this newly-discovered truth. Yoga textbooks were filled with advertisements of American New Thought, and vice versa. Vivekananda's work, Raja Yoga, was also filled with inspirations coming from metaphysical New Thought. What's more Y. Ramacharaka, in Hatha Yoga, also referred to this way of thinking. If we send a positive thought to a given body part, we can cure ourselves from sickness (ibid.: 130-131). Autosuggestion and affirmations are here a very important element in the work with the mind. Sending signals to the body is connected with work on the body.

A different reason for disregarding the resemblance of yoga with hatha-yoga was the then-negative image of yogis in the West, and also in India, as mercenaries or those who took fees from travelers in exchange for protection in the $18^{\text {th }}$ century. As has been mentioned earlier, they were perceived as beggars or acrobats giving out cheap, public entertainment.

Only when asana practice was joined with the Hindi nationalistic discourse did the situation change. Hindi elites wanted to educate a healthy generation of its "intelligentsia," and in the beginning used Western gymnastics to do this. Gradually asana practice was included in the traditional Hindi physical culture. Physical culture became well-mannered politics for many patriotically-oriented Hindi people, as they tried to overcome the image of a Hindi person as a physically weak man. Both in India and in Europe at this time, modern physical culture was at the center of interest of the eugenics movement, and social Darwinism was also popular. According to the rules of eugenics, it was believed that the physical development of an individual could, thanks to body exercises, influence the next generation through the possibility of inheritance by children of, for example, a father's achieved physical strength. Hindi nationalism incorporated ideas about the development of physical culture and eugenics and mixed them with yoga practice. 
In this way it was possible to change the image of yogis from "hidden terrorist agents" (according to the British) to freedom fighters for the Indian national cause (according to Hindi patriots). Travelling patriots often taught martial arts under the name of yoga in order to avoid arrest (ibid.: 103-104). This hidden form of martial arts teaching resembles the story of the Brazilian capoeira, which developed as a dance. Yoga developed constantly, joining together different plots and traditions: gymnastics, martial arts, and asanas from the Hindi tradition. In the beginning of the $20^{\text {th }}$ century, it was in a constant change and fluctuation. One of the traces of this discourse was that not only were the roots of many Hindi physical exercises underscored, but also sports such as hockey, cricket, tennis, billiards, and boxing (Indian System, according to K. Ramamurthy). Even this new nationalistic system of training one's physical strength was called yogism (ibid.: 108). Indians also wanted to discard British gymnastics, as it was part of the physical culture of the colonizers. The attitude of the educated Hindi society that had, up until then, thought that physical exercises were for illiterate people and not for educated Hindis, gradually changed (ibid.: 111).

Singleton's observations about the influence of female physical culture on the popularity of hatha-yoga are also interesting. This culture was based on performing postures exhibiting the aesthetic aspects of the human body, as well as on postures stretching the body and showing its flexibility and litheness. Hatha-yoga postures were really close to female gymnastic positions, which made it easier to join them together in the popularization of physical hatha-yoga. The esoteric dance of François Delsarte (1811-1871), accenting the co-ordination of the voice, breath, and body gestures, gained great popularity in Europe and the U.S.A. In this system, later improved on by Genevieve Stebbins, each spiritual function has a parallel given body function. Here we are dealing with the suggestion of embodying spirituality (ibid.: 144-146). Current hatha-yoga practices resemble the gymnastic exercises designed especially for women in the first half of the $20^{\text {th }}$ century. Exercises designed, for example, by Cajzoran Ali were based on some system of understanding how the human body works, where certain glands in the human body are responsible for harmony of the contact with God, who actually resides in the individual. Spirituality was present in female gymnastics long before it was connected with yoga and its place, for example, in New Age movement (ibid.: 148-149).

The main person responsible for the current shape of yoga in the West is its great Hindi propagator and teacher T. Krishnamacharya. Residing for 20 years in the Mysore Palace (India), where he was supported by the reigning Maharaja (Maharaja Krishnaraja Wodiyar IV), 
he designed a yoga system in which the main aspect was a rigorous (often acrobatic) series of asanas designed to be performed in a given sequence. This period of his teaching most influenced his students, first Sri Pattabhi Jois and next B. K. S. Iyengar, who slightly modified Krishnamacharya's system by limiting the number of acrobatic elements. After leaving Mysore, Krishnamacharya still taught yoga, but there was less emphasis in his system on the arduous acrobatic elements (which can be seen among his students of that period, for example, his son T. K. V. Desikachar, and A. G. Mohan) than in Pattabhi Jois' teaching.

Already at that time, in the Mysore Palace it was decided to popularize traditional Indian sports and physical culture together with asanas. European styles of gymnastics were also taught there. Krishnamacharya's style of teaching appealed to the Indian culture and its tradition. This is also where the series of asanas called suryanamaskara (the sun salutation) were created. According to Pattabhi Jois, it comes from the sacred books of Veda, although this is difficult to prove. In general, Singleton assumes that this cycle of exercises was designed in the period of Krishnamacharya's teaching in Mysore (ibid.: 180, 221-222). The lost traces of asanas and their sequence are very interesting. They allegedly come from an old book, Yoga Kurunta. However, no one has ever seen the book apart from Krishnamacharya, as it was supposedly lost. Maybe it never existed and reference to it is only for legitimization purposes. It is also similarly difficult to confirm the sources of modern hatha-yoga (the Ashtanga Yoga System) in the sutras of Patanjali, who is believed to be the creator/exegetist of the whole hatha-yoga system. Singleton claims however that this is rather projecting the interpretations from today's perspective into the classic Indian texts. It also needs to be underscored that in modern yoga the series of satkarma techniques (so-called cleansings which involve cleaning the stomach by swallowing string, a special form of enema, cleaning the nose with water, looking into the light of a candle up to the moment of crying in order to cleanse the eyes, massaging the stomach, and breathing the air out by cramping the stomach muscles) were almost completely erased. It is worth noting that the name Ashtanga Vinyasa was used for the first time after the first American students came to India in the 1970s. Earlier, Pattabhi Jois called his classes asanas teaching.

Yoga practice was not a strict system. Krishnamacharya often came up with modifications of asanas, or adjusted them to the practicing individuals. According to this master, yoga practice has to be adjusted to the period, place, and specific requirements of a given individual (ibid.: 188). The acrobatic and rigid version of repetitive sequence (vinyasa) in hatha-yoga versions, represented by Pattabhi Jois, is thus 
the only version taught by Krishnamacharya. It needs to be mentioned that the dynamic and quick passages among vinyasas - which last to five breaths in Pattabhi Jois' system - come from the period when Krishnamacharya popularized hatha-yoga and his students gave dynamic shows with their acrobatic and most imaginative asanas. These shows were held for the Maharaja's guests both in the Mysore Palace and later on in other places, including in the 1930s, especially during Krishnamacharya's propaganda trip to the south of India ordered by the Maharaja.

For sure, Krishnamacharya's teaching concentrated on the physical side of yoga (asanas). This aroused criticism of his system. Singleton believes that Krishnamacharya's system is "a synthetic revival of indigenous exercises (comprising yogasana alongside other types) within the context of Westernized curricular physical education in late colonial India" (ibid.: 198). When he began teaching in Mysore, Krishnamacharya inherited a yoga room there (yogasala) together with its Western equipment (e.g., ropes for climbing). This equipment was used by him in this phase for physical exercises. Later, he moved away from using expensive gymnastic appliances and ran his classes without using such things. Many asanas that he introduced were similar to the exercises used in the system of Danish gymnastics by Niels Bukh (1888-1950) (ibid.: 200). During this time asanas were introduced pragmatically to the gymnastic system of teaching. The sequence of suryanamaskara (consisting of squats and exercises similar to pushups) can be treated as a modern, physically-oriented performance of the ancient practice of "bow before the sun" (ibid.: 206). The modern renaissance of yoga can thus be viewed as a conscious program of modernization of an ancient tradition.

The creation of modern physical culture is surely connected with intersecting and cutting into the past borders of many worlds and intellectual trends: para-religious spirituality, Western esoterics, the world of medicine, health and hygiene protection, chiropractics, osteopathy and other practices on the body, psychotherapy concentrated on the body, the modern rebirth of Hinduism, the socio-political rebirth of India, etc. These worlds and the phenomena happening within them influenced the creation of modern hatha-yoga in Western societies. The criticism directed against the modern yoga practiced in the West - that it lacks spiritual elements and often obscures the fact that the so-called "traditional yoga" currently in vogue came to life only at the turn of the $19^{\text {th }}$ and $20^{\text {th }}$ centuries, as well as criticizing hatha-yoga as a form of fitness - does not acknowledge the fact that physical training can be treated as a spiritual practice, which has a place both in the Western 
world and in India. In light of this fact, in the context of the historical development of hatha-yoga one cannot be astonished that today it is treated by many as sport or that Bikram Choudhury, who organizes yoga championships in the performance of asanas, tried to negotiate its entry into the London Olympic Games.

Should such interpretations of yoga evoke surprise? They change over time. The translations of different classic texts usually conveys the spirit of the time that the translation was done. The translations are done within a "frame of beliefs and faith" (belief framework) that includes personal, social, and cultural assumptions through which the individual reads his or her own world. This was the case with yoga and its classic texts in India in $19^{\text {th }}$ and $20^{\text {th }}$ centuries, and in the translations of them done in the West during that time. Yoga interpretations and asanas practices are translations from the point of view of modernity and, according to Singleton, this is what was happening with yoga during the last 150 years. The search for medical and scientific justifications for the benefits stemming from yoga practice is an example of usage of a "belief framework" from a given historical period (Singleton 2010b).

$$
* * *
$$

I have conducted a broad examination into the views of Marc Singleton as I think they can stand in opposition to the beliefs of persons looking for deeper spiritual values in yoga. I asked two specialists and long-term hatha-yoga practitioners what they think of Singleton's assumptions connected with the roots and origin of modern hatha-yoga (Singleton 2010c). One of the experts was Michał Szczepanik, an experienced teacher and the owner of a hatha-yoga school in Lodz (Poland) - "The Center of Hatha-Yoga of Michał Szczepanik." This is how he replied:

I agree with Singleton that the question about the authenticity of yoga is not the most important question, and maybe it is formulated wrongly to start with. It is not possible to state when yoga reached Europe and how, for example, gymnastic exercises from the turns of previous centuries, even unconsciously, could be inspired by some echoes about the knowledge of asanas. What's more, many inventions were in the times created, forgotten, and again created without any previous consciousness of former traditions. People have similar bodies, I am not astonished then that they can, based on their observation, reach similar conclusions about the way to keep them fit. I, myself, when I was young, stretched in a way that as I have later discovered resembled asanas. Many children sit in badhakonasana even though they have never practiced yoga and maybe never will. 
Asanas are only a part of yoga tradition. They were supposed to be a preparation for the body for practicing pranayama, contemplation, and meditation. However, in my belief, only through physicality could yoga enter the Western culture. Even in Poland, at first yoga was treated with suspicion, as some sort of a sect. Underlining its physical aspect and its beneficial influence on health allowed for popularizing it in the first place. The differences between the Indian and Western European perception of the body and the understanding of spiritual development is a topic for a different conversation. I think that the difficulty could already appear in the attempt to precisely formulate it. As Singleton writes, India was not free from the influence of the Western thought and mentality (what is also very visible in modern India). Seemingly thus, the obvious division between Indian thinking and Western European deeper thought can lose its clarity. And, if I refer Singleton's thoughts to Poland, should we treat our country as Western European? Much stronger than in the West, even until today, is our way of thinking in religious categories that on one side can strengthen the need for spiritual searches, but often become the source of many prejudices or easiness in performing guru postures.

More interesting, and at the same time more difficult, than the question about the authenticity of yoga is the question about the aim of practicing asanas. According to ancient tradition, asanas were not an aim by themselves and were not supposed to improve the mood. They helped to prepare the body for deepening the breaths (pranayama) and proper sitting in meditation was supposed to strengthen the nervous system and thus help in going through the next stages of broadening awareness. Even the notion of health was treated as an element of spiritual development. The illnesses that asanas were supposed to prevent were treated as one of the main obstacles on the spiritual path.

And at the same time, yoga in the Western world, including in Poland, has become a business that encompasses not only courses, teachers' courses, but also the sale of accessories for exercising. I see nothing bad in this; I have a yoga school myself. However, very often one can lose the difference between yoga and fitness. People in some way buy the means to improve their mood or silhouette. This has its good sides. The fashion for yoga attracts a lot of people to these exercises. For some, asanas can become gymnastics, for others the beginning of deeper changes, an incentive to observe oneself or start to take an interest in Indian philosophy. But it also should be remembered that reducing asanas to only a healthy way of spending leisure time is no longer yoga. The price for the popularity of yoga is the need for an individual search for those who treat yoga, according to the tradition, as a way of development. Exercising everyone has to answer the question why he or she does it. If I want to sooth the pains in the back, it is sometimes enough to attend a few classes a week. If one wants to achieve inner peace, it would probably be necessary to introduce regular daily practice, and often a change of life style as well. And finally, if someone treats yoga as a spiritual practice, he/she can connect it with the practiced religion. That is at least in my understanding, but I know that the attitude of the Catholic Church to yoga is somewhat distrustful, and that many Indian spiritual masters, and I do not mean Vivekananda, but in modern India, would then consider doing asanas as empty gymnastics. Unfortunately, often those who practice yoga lack the professional guidance of a reliable guru. I consider myself to be an experienced asanas teacher, but not a spiritual master. I can point out the beginning of the road to somebody who wants to continue looking for strict spiritual guides. Some- 
times, it can turn out that yoga is the beginning of difficult confrontations requiring further work with a therapist.

Coming back to Singleton: I agree with Singleton that the yoga we practice in the modern world very often has little to do with the real spiritual practices of ancient India. In my own experience there is a strong belief that asanas are a very useful tool in personal development. I myself started practicing yoga to stretch and be able to climb better. So I was interested only in the physical aspect. But as a result of the practice, I completely changed my life style and my system of values changed immensely. In my own case, I perceived many surprising effects of these strange looking twists and turns. Still, I know this does not happen in every situation. Some become disappointed, after longer or shorter periods of time. Myself, even owing so much to asanas, I do not treat them as the only and reliable means of development. We live in a world of individual decisions and individual responsibility so practicing yoga does not matter if we treat it as a very old tradition or a relatively new invention. In the end I guess depends on how one wants to use it. (electronic correspondence with Michał Szczepanik)

The above interpretation of the controversy over the source and origin of modern hatha-yoga is pragmatic in the philosophical sense, but also practical in the sense of the everyday work with yoga that each practitioner has to engage in. It is important to perceive the changes in one's body and mind and the changes in the meaning of yoga even while practicing asanas. From this point of view, it seems that meanings should be given to the elements of practice in order to practice hatha-yoga, and build the sole motivation for its practice. One can even conclude that it does not matter what meanings of hatha-yoga these will be. The practice of hatha-yoga is, according to Michał Szczepanik, a development; a process. One can agree with this assumption under the condition that asanas are only a part of the practice and that they should not be treated as the essence of yoga. They only start the true spiritual search.

Below is another statement of a hatha-yoga practitioner, also regarding Singleton's statement that hatha-yoga is a modern creation of our civilization. My question was: Do you agree with Singleton and the leading idea that the yoga we practice today was and is under the influence of Western gymnastics? Karolina Gołaszewska replied as follows:

I will answer perhaps in a more yogi style than a sociological way because I believe it needs to be talked about.

I absolutely agree with Singleton. I believe that yoga evolved and changed completely from the time of its creation. And now, a very metaphysical answer from my side - the whole world evolves and changes its face. The Earth and its inhabitants enlarge their energy potential, they change it and create it from the very beginning. History, including the one presented by Singleton, shows that yoga teachings, meth- 
ods, etc. were changed by its creators on many occasions, even in the distant times, and there was never such a thing as one truth or one way of teaching.

I think that this is a point that yoga teachers do not want to agree with. The ones who are more intelligent repeat that it would be enough to sit under a tree opening the self and then we would not need all of these asanas.

As a person doing hatha-yoga in ashtanga vinyasa style, I can say that we should choose the practice that works for us. If we feel improvement in our inner development just by gardening, we should continue this. If we feel inner changes by putting our legs above the shoulders, we should continue this.

Yoga shows how to turn off the logical mind because what it has to offer goes way further back, outside our capabilities of perception. It teaches us to use this part of the potential of our brains that we do not use on an everyday basis. Yoga and connected studies show humanity that to be true we are in a cradle, and there is a long way ahead of us. (electronic correspondence with Karolina Gołaszewska)

The above statement is, just like the previous one, a type of pragmatic reaction to Singleton's interpretation that the origin of yoga as a practice was under the strong influence of different systems of Western gymnastics. If something helps and suits the individual, it should be practiced. What's more, one can see here a vision of historic reality as a process. The practitioner does not assume that some systems of knowledge are inherently stable and can be transmitted from generation-to-generation according to the intentions of the first creators of the system. There is no one and unchangeable truth. Every system of knowledge undergoes interpretation and changes over time, becoming redefined and changed. So too the practice of yoga has changed. The practitioner herself does not see any problem with Singleton's thesis regarding the origin of yoga, although she sees that such problems can arise with others who may not accept the thesis about the Western origin of modern hatha-yoga practice.

\subsection{Conclusions}

The social world of yoga is divided into many social subworlds according to various ideological divisions. The social subworld of hatha-yoga intersects with many other social worlds. The modern Western world is very commercialized, making it difficult even to imagine that the world of yoga was ever free from the influence of the world of business. The modern practice of yoga shares many common grounds with this commercial world (production of accessories, using yoga for healthy food ads, development of yoga schools as businesses, yoga workshops for company workers, etc.). The same intersecting is 
evident in the world of conventional medicine, where yoga enters as a full-time participant of physiotherapy. Here one also sees the legitimization of this world, since official certificates are issued to yoga physiotherapists. Mass culture also often uses yoga for its own aims, where its popularity is linked with other promoted actions, for example, certain types of music. There is disagreement within the world of yoga over whether it is supposed to be a field directed toward physicality (the physical development of a person) or whether it should also be directed toward spiritual development. Spirituality is only emphasized to a small degree in the Western world, and this too is due to commercial reasons, as well as the origin of hatha-yoga. Hatha-yoga was developed based on the Hindi yoga tradition, but also taking elements from Western gymnastics and esotericism. Yoga, in Western societies, blends in perfectly with main trends and life styles. A healthy life style, healthy food, and physical exercises are values that are common to both worlds, even if yoga did not develop in parallel with the development of these values in the Western world. And for some parts of Western society the spiritual and religious aspects of yoga are also attractive, even if they do not dominate in modern yoga, since they were not present from the beginning of formatting yoga based on exercises and performing postures (asanas) even in India. In addition, aiming at the spiritual traditions of Hinduism and yoga can create a collision of worlds with modern institutional religions, although this can be avoided by a syncretic approach integrating many beliefs and religious practices. Yoga, even in its physical, Western form, can also have a spiritual aspect and can, for example, push one towards or inspire a spiritual search, and itself be a spiritual practice. By taking a pragmatic approach to yoga practice it is possible to practice asanas without taking into consideration its entanglement with business or gymnastics and sport, and even these types can have spiritual meaning. 


\section{CHAPTER 3 \\ Commonsense definitions of yoga and its meaning for practitioners}

\subsection{Introduction}

In this chapter, we introduce commonsense definitions of yoga. How is yoga defined by the practitioners themselves? What is it for them? What role does it play in their lives? In the life of an individual, definitions of situations and ascribing meanings to the objects of the "outer" world are very important. Yoga, being a set of practices, cannot enter the world of the individual without having defined meanings that the individual ascribes to it. The activeness of a practitioner in the world of yoga depends on the importance that the individuals ascribe to these practices and what they mean to them in their lives. The more meaning an individual ascribes to the practice of yoga practice, the more he/she engages in it and remains in the social world of yoga. The interpretational work is, of course, done individually, but the social surroundings and the dominant interpretational frame connecting the body and health in the modern world are of great significance in the process. This frame in the social world of yoga is built upon the classic texts by yoga masters. The language used in describing the experience of yoga practice has already been shown. This language co-produces the experience, including the bodily feelings. Language classifications are acts of placement. Definitions help to place an object in particular categories. Is yoga a physical activity or a spiritual one? By placing an object, often by defining it, we obtain directions for our actions. Language classifications are also evaluations of the objects of perception. The value is an evaluation of an object (Strauss 1997: 23-25). The value of yoga is not in yoga itself, it is in the minding of and evaluation of yoga.

The interpretations presented below are an example of commonsense theorizing in the context of defining basic activities. Theorization is supposed to legitimize the social world, mark its independence from other worlds, and give the motives, voice, and justification for taking part in this world. Behind these significant components there are others, following and accepting the knowing perspective of the masters in defining the basic actions and assisting in a given area of 
reality, ascribing them specified meanings. Commonsense theorizing is a derivative of the work by key theoreticians of a given social world. It fulfills and closes this world, constructing its boundaries.

However, we should remember that things in the world change: "The naming or identifying things is, then, a continual problem, never really over and done with" (Strauss 1997: 27). This happens especially with work on body, when we cannot always express bodily feelings.

\subsection{Individual interpretations of hatha-yoga}

Despite the fact that there are many formal definitions of hatha-yoga (see Chapter 1), practitioners often try to define what yoga is on their own. In interviews they describe many features connected with the definitions of yoga, however its actual understanding by practitioners is often ambiguous. One can say that each person practicing yoga has his or her own definition. This results from their individual motivations to practice yoga, past religious socialization, past experience connected with work on the body, and search for other experiences or spiritual practices. The variety of practices derives from a different source. With respect to yoga, it's important to note that the individual approach to yoga and its definition changes over time, together with the development of the practice, and gives a full understanding of the practice:

I have been practicing for fourteen years. And my approach changes all the time, one can say every minute. That is because yoga is not some mental assumption we try to reach. Yoga is not a philosophy. Yoga is discovering. That is why I am astonished here every minute. But always astonished in a positive way, in order to not be attached to what we expect or expected from our practices. So we should be ready for what yoga is giving us. I thought at the very beginning that the perfection is within arm's reach. It seemed that it would take me two or three years and I would be able to fully control my emotions, my physical body, as well as my mind. It turned out to be more distant than I anticipated in the beginning. But this aim is somewhat more beautiful. Now, I have a different perspective than when I started, because when I make a step forward... this aim seems to be a bit further away, but I see it from a wider perspective, as if more from above. And, the further I go, the more the horizon moves, and I discover more and I understand more. (practitioner for 14 years)

Yoga is often defined by practitioners as a work on calming the mind by exercise and work on the body. Thus, the commonsense definitions of yoga are connected with yoga affecting the body and mind of the practitioner, and these are the most common definitions. 
From the formal point of view of the practice of yoga is, among other things, a way of working on your body (see: Iyengar 2005a). This is also how the practitioners see yoga ("You are satisfied with it when you work on your body and think how much more you can do" [practitioner for 5 months]). But, as has already been mentioned, this is work on the body which is closely connected with work on the mind.

In the work on the body, there is no competing with others. Yoga is a strictly individualistic practice, even though it can be done in a group. Work on the body is to be in the posture (asana). There is some form of meditation in a movement, which is sought after by many people. An interesting language idea often used to describe the practice of hatha-yoga practice is "liquidating/breaking body blockages." This term is also used by yoga instructors. Body blockages can also be described using the "shell" metaphor. The interviewee quoted below also gives the stages of "the work on the body": 1) liquidating the shell; which is liquidating the pressure connected with body tension in its different parts; 2) learning how to breathe; 3 ) calming the mind. Only at the end of this road is there an inner peace:

And here the greatest art is to, err, not to endure in the movement, here is not about endurance but about being in a given posture. First are those corporal, and these mental or physical exercises lead to, err, liquidating blockages that exist in the body. And, to be honest, there are many of these blockages because, err, we are not aware how we shell ourselves with our body. We create a shell from this body. In order not to show emotions, we often withhold ourselves; we often do not show that we are unhappy or that we are too happy and we do not cry so easily because we manage all this in tension. We shell ourselves. On one hand, we do not let outside what is inside; on the other hand, we shell ourselves so no one will hurt us. So that it would not be so easy to hurt us, so the emotions that others show to people around us did not reach exactly us. And you first have to destroy this outer shell in order to learn how to breathe... correctly. In order not to withhold the breath, for example, from fear... or shock. So, when in the middle of a very uncomfortable situation, which happens to us on every step, know... in spite of tension which is in us, outside know how to breathe. Because breath is life. If we stop breathing, we stop living... Then, when we know how to relax, when we know how to cope calmly in every situation and manage our breath and breathe calmly, then we can start calming our mind... And inner peace cannot be reached without relaxation, without getting rid of the body blockages. Please, believe me, everybody has such blockages. (practitioner for 4 years, age 46)

According to practitioners, the work on the body leads to cleansing both the mind and the body:

- And you know what - you leave the problems somewhere, somewhere behind you. You enter, you enter this yoga with problems and you exit really cleansed. It sounds 
really weird, but this is the truth. I do not know where it comes from, maybe from this relaxation in the end, maybe because it pays attention to what you do and fully focus on it so your leg, for example, would be at a right angle, etc.

- And what do you mean by the relaxation at the end? Is there a different kind of exercise, or...

- Well, yes, this is what I told you earlier, but you misunderstood me (laughter) or I did not make myself clear. It is that instead of such meditation that I did in my previous class with a different man, that this woman introduced, well it is a standard thing that after class you need to rest, which means you lay down on the floor in some proper position, adjusting yourself in such a way that you breathe with your diaphragm, so you need to lay down properly, you have your blanket put underneath your head, and this is such an open position, you have your hands on the floor and this woman puts your mind to such a level as if you were lying in grass, looking into the sky, clouds showing up and disappearing, and you think about nothing at this moment. But you see as the clouds float, and thanks to this you relax. And you must not think about anything, the woman then repeats that this time is only for you. And you leave these problems somewhere and leave so cleansed. And you should not eat neither before nor after yoga... (practicing student of ethnology)

For one of the interlocutors, yoga is a kind of independent work on the body and mind, a type of meditation and silencing. It is also a philosophy and way of life as well as a mind therapy (sometimes it is called mind regeneration). The peace achieved through yoga can be transferred to other spheres of life, for example, family life. Yoga is work on emotions. "Peace and relaxation" - these are two key words necessary to come to some common definition of yoga:

And besides, I believe that yoga is such a kind of work with the body and mind that the human needs to do on his own, it is some sort of meditation and silencing, thus the contact with oneself is sufficient... Yoga is not only exercise, body training and physical fitness, but also some philosophy and way of life, that is why this peace and relaxation I feel in yoga I try to transfer to everyday life, to stay calm in contacts with family, friends, and other people.

Yoga lets me concentrate only on me and my own emotions and thoughts - it is some sort of mental therapy for me. (advanced practitioner)

Calming the mind is nothing other than what Iyengar and Patanjali called "restraint of the movements of consciousness" (Iyengar 2002b: 82). There is, thus, in the commonsense definitions a reflection of formal definitions of yoga coming from the masters. A commonsense discourse of yoga comes from the formal discourse of yoga and is transformed to more simple formulas. 
And what is yoga for me? It is something like mind regeneration for me. Such regular yoga practice influences more efficient work of the mind, and thanks to this I feel that I can use it more effectively. (practitioner for 5 years)

For many yoga becomes, through the ability to concentrate, a skill in recognizing your own needs, and helps one become happier, not only in class but also in everyday life:

- What do you advise when somebody comes to the class? How should he start, act, and what should she pay attention to? What, apart from exercises?

- In class, he or she should mainly practice, listen to the commands of the teacher, feel good with oneself, be happy that she or he is in the class and taking part in the practices, and in all of this find oneself, because we simply, somewhere along the way, lose ourselves and we become scatterbrained, chaotic, and we do not cope with our own emotions, fears, with everything that surrounds us. And yoga is supposed to help us direct this concentration on ourselves, recognize ourselves and our needs, and teach us how to cope in life and how to be happy, 24/7. No matter what happens around us, no matter if somebody dies, no matter if, if there is some tragedy. Yoga teaches us how to be such a blade of grass, very resilient to all storms. (yoga instructor)

\section{Very often yoga is treated as a purely physical exercise, gymnas- tics, or even as sport:}

The same thing is with yoga. Yoga is a sport, call it a sport; I do not call it sport because this is not sport, this is a form of relaxation that has no developmental boundary, but we assume such a statement and we treat it only as a form of relaxation to help the organism stand up to getting older... however, I really treat yoga only as a form of recreation, gymnastics, and I laugh at such yoga practice "just because" it gives so much positive effects, but I do not want to connect yoga with this whole surrounding, you know, spiritual because I have not grown up to this yet, or maybe I will never grow up, maybe this does not interest me, and maybe one day... (beginning practitioner, age 50)

In common yoga definitions, there are often suggestions that yoga is researching oneself, one's own body and mind. In order to do that you need to pay attention to the technical aspects of its practicing, for example by focusing on the role of breath in order to control the emotions and mind:

May I adjust my breath? May I adjust my emotions? May I adjust my head and my soul? This is what the practice is about, that is why it is called "research." Yoga, yoga is a science... in which we research ourselves. It is not that I walk and... many people think so, that I go to yoga class, exercise and it is fun, in fun clothes, and I will see all of my friends. Madonna also practices it; it is super cool, I will also practice yoga. There are many such ideas because it is popular, fashionable, and super, and it can be. 
But... there is something like, well, that yoga is so old and it is not something, like something that is trendy, but it was already here for 5000 years. This is what people do, they do research on themselves. I research myself, the way I am. And I research if I can change. But first, I need to control my body, need to control my breath, need to control my emotions and my own head. (practitioner for 12 years)

There are often suggestions that yoga is an individual practice and that there is no need for words to understand it, but that it needs to be felt in practice to see its effects:

I rather prefer when the instructor does not talk too much during exercises about what is happening with our bodies... I prefer to feel it myself and decide myself what is happening, what I am experiencing at the moment, during performing each position. (practitioner for 5 years)

For this reason, in the research visual methods were used (interview based on a movie, analysis of video recordings and photos of yoga practice) in order to reach the essence of the features of the definitions of yoga that are happening outside our verbal language, in the action itself on the bodily level (see Chapter 5).

The fact that yoga is an individual practice is underlined also in other aspects, that is, that you do not need any help or support from friends or peers. Interlocutors below use the metaphor "lonely trip" and "inner trip," pointing out that no one from the outside has access to what the practitioners feel.

- You practiced yourself? That is, with no support from friends and other people?

- Eh, no support from friends. For me, yoga is such a lonely trip, the presence of an accompanying person is absolutely unwelcomed. That is why because other people are simply disturbing, going to yoga I choose contacts with yoga, not a friend, with a friend I prefer to meet in a restaurant, at my place, cook something delicious, some dinner and eat while drinking wine, and yoga is only for me, not for the person next to me. Well, practicing people see it themselves, that when in yoga class, of course the surrounding is very important, because before yoga or after yoga it is nice to sit, talk, have tea with people you go with, but it is, to be honest, a lonely trip. I do not invite anybody. There is no need. (yoga instructor)

We are never bored, that is why every time when I enter my inner world, I discover something new and it is like an inner trip, discovery, new adventures. (practitioner for 14 years)

Meanings ascribed to yoga often show the strong influence of yoga on the individual and his or her functioning in life. They cannot imagine life without yoga, they even use the metaphor that "yoga is the mother", and apart from that that it is a lonely practice. This applies 
to advanced practitioners, those who moved on to stage three of the practice, to fuller recognition of the spiritual aspects of yoga practice (see also Chapter 4):

- What is yoga for you?

- Mother. I treat yoga as a mother, and always when I talk to other teachers of yoga, then everybody sees in yoga something different. For me, yoga is a mother, I think about it like about a mother. Just as you think about, I don't know, about Mother Earth... well, that is how I think about it and sometimes in difficult situations I feel how it cuddles me and pats me, a very deep emotional attachment. Every asana carries different feelings, a different experience. This is why this is a trip that has no end. It always starts from something different, it can start from fashion, people go to yoga because, for example, it is very fashionable now, so it depends on fashion and if they do not find anything apart from fashion, then they quit yoga very quickly, but if they can take something out of it, then they stay with it for many years, so the reasons for taking up yoga can be different. It is, I believe, a lonely trip, a trip towards yourself, inside your own body, a trip that has no end. (yoga instructor)

Next, the same interlocutor gives us to understand that yoga can be an autotelic value ("practice is the aim on its own"), and it is performed for its own sake. Yoga spreads into your whole life, which thanks to the practice becomes meditation. Attention, the ability to perceive the outer and inner world, are the effects of yoga practice, although it is not planned and should not be gained at any price:

- What should yoga give? What does it give you?

- What it should? I think that it should nothing, everything that happens there is a side effect, it is not a goal itself, only the whole process is the greatest prize, so the road that we are now following, so the one who goes to yoga and would like to gain some goal, then he searches in the wrong place. The practice itself is the aim in itself. And the stages of this practice are different and different people come across something different. They overcome their weaknesses, learn something new about themselves, we become observers not only of these outer structures but also these inner structures, that is your mind, your body, your breath. We learn attention, prudence; our life can become meditation...Well, yoga is not only exercise, of course... well, as I have already said earlier, yoga is first and foremost a study of consciousness, attention, inner sight into the surrounding world, contemplation and meditation in the everyday life, and such every day, 24 hours a day. (yoga instructor)

Yoga is rather not control over the body and mind. It is about gaining consciousness, insight into one's own emotional states, so then all the problems can resolve themselves: 
One needs to understand the main mechanisms that function in the world, how our minds function, what nooks... er... traps, blinders it puts on us, and when we know about it, we know these mechanisms, then it is much easier to control oneself. That means that control, such strict control as all yoga teachers were saying not to control, because it will not do anything, you can somewhat subjugate it, but it will come out of us anyway, so cope with it, don't control it. So the key to everything is the consciousness; we choose control through consciousness. We do not tell ourselves, for example, that "I am nervous and now I will become calm because I practice yoga. So if from today I practice yoga, then I will no longer be nervous but calm..." Well, this is an utter lie, no one will manage that... well, there is no such thing. It is about learning about oneself, feeling your own inner consciousness, so realize that: "I am nervous because..." and find the source of this nervousness. Not to suppress it but be aware of it, and then everything solves itself. (yoga instructor)

Often in these common descriptions and definitions of yoga practice there is a reference to yoga in the context of what it offers in relation to the body and the mind in Western culture. This happens in the context of yoga delight.

\begin{abstract}
That is why yoga is wonderful, because it does not tell us to change, but tells us to love ourselves the way we are and be conscious. The Western culture orders invasions, injects a substance, enlarges breasts, do some liposuction... still look beautiful, still be twenty, yes; but we are not, we will become more and more mature women and I believe that there is nothing more beautiful than wrinkles that are the diary of our life, all of what we experience. For me, youth is in the head, in the eyes, palms and feet. These are for me the most important parts of the body that radiate with youth. If they are young, then we are young. If there is no radiance in the eyes, if the feet and palms are numb, then no matter what and how much and what you inject in yourself, or do I know not know what else you would do with the rest of your body, you are still old. (yoga instructor)
\end{abstract}

The meaning ascribed to yoga depends not only on the level of engagement of the practitioner, but also on the emotional attitude towards the practice (e.g., delight). Yoga also causes the redefinition of body beauty. The body is beautiful, young in its fitness, spirituality emanating from the eyes, and not only from the body itself. This is a completely different conception of defining the body. Yoga can change the way of perceiving the body, it is different from what the Western culture offers, even though yoga is used here to realize the values of Western culture.

Some of the interlocutors underline the importance of meditation in yoga. In general, yoga can be treated as a meditation in movement. What's more, one of the asanas always used to end hatha-yoga sessions, shavasana, resembles correct meditation and is somewhat of a preparation to meditation without body movement. Some instructors 
introduce full meditation as an element of yoga practice, and some of them concentrate only on physical exercises. The interlocutor quoted below emphasizes the presence of a bond with other persons meditating within a group:

I went every week to these meetings and I started liking it, especially this part that at the beginning, which I laughed at the most, which is meditation. I tell you, it turned out to be something amazing. You sit in a group of 20 people, quietly calm yourself, nobody says anything in the beginning, and you breathe... like at the doctor's. Breathe in... breathe out... breathe in and breathe out (shows deep breaths in and out). Nothing difficult. And when you breathe like this, then slowly you feel some strange bond with these people that sit there, an amazing feeling. All strangers you did not know earlier and you feel somehow ok, safe, and even cool. You do not think about anything else, studies, exams, boyfriend, parties, you just only sit like that, an oasis of peace. You have any idea how much I relax there? I tell you, you need to try.

[The instructor] says that our bodies are tender and passive and will be subjected to meditation, and we will enter a dreamlike state. I always wanted to laugh at that, but it's true. I feel like some shaman at a spiritualistic meeting (laughs). But seriously, it is super. You cannot describe it in words, even more because you look at me so strangely when I talk about it (laughs again). (practitioner for 3 years)

There is a strong connection between hatha-yoga and meditation. This aspect of defining yoga is also strongly emphasized by yoga masters in their works defining hatha-yoga. Asanas are supposed to give "emotional stability," "they relax the person totally" (see: Iyengar 2005b: 267). So some indicators of what yoga is can be found in the basic works of modern yoga masters. Yoga understood in such a way gives peace and emotional stability:

So, one can say that meditation is strongly connected with yoga because, first, it is during the exercises and, second, that there are pranayama-breathing exercises. And the next stage is meditation, so it is connected somewhere. And in the same way meditation influences the body - gives emotional stability and emotional stability gives energy for life, right? Because you do not lose energy on pointless thoughts, sorrows, on some strong emotions. You are more balanced, calm, and less prone to stress. These are such physical aspects. (practitioner for 5 years)

\section{As has already been mentioned, the practice of asanas can be treat- ed as a meditation in movement:}

Practicing yoga regularly, approaching the same postures, it will change, we will be able to make more complex postures with greater comfort, until we reach such a state where people ask: "Well, yes, we do it here, stretch, flex, these postures, yes... and what about meditation? Why do we not meditate?" I say: "How do we not meditate? We meditate in every position!" Although we do not sit in the corner just 
only being in a position, if we overcome it, then the stage of meditation appears. The body stops being an obstacle, stops pinching; can appear in any position if it's calmed. And this is how we meditate in movement. (practitioner for 14 years, yoga instructor)

The terms used here by the narrator come from the works of Iyengar, who is the master for this yoga instructor. Iyengar writes about "active meditation": "Through the performance of asanas, I become totally involved and find oneness of body, mind and soul and for me it is active meditation" (Iyengar 2002b: 149). ${ }^{1}$

The terms used by yoga masters are assimilated by yoga instructors and passed on to the practitioners, often in direct conversations. The practitioners themselves, at the bottom of this ladder, are often not aware of where these terms come from.

Some of them define yoga in opposition to those meditating in a static way, underlining that this is hard work and not only sitting:

Because it is not so that we sit and meditate. Well, I mean, there are, for sure, there are some classes and other teachers, but here there is hard, physical work. (practitioner for 8 years)

The pressure to meditate shows the "spiritual aspects of yoga." Spirituality, of course, means different things in the modern world. One can point out in yoga practice the search for contact with the "soul," or "true ego," but one can also speak about "satisfaction." This is one of the many keywords to define the "para-religious" aspects of many spheres of life in the modern world. Yoga then also has an autotelic value for an individual.

- And what is yoga in your life? What place does it have, what role?

- Yoga is, to be honest, the only thing that gives me true satisfaction. There are many pleasures in life that give momentary satisfaction or satisfaction (looking for a word) that has nothing deeper behind it. However (putting emphasis), yoga is gaining the perfection and the sole fact of following it gives satisfaction every day. And every move that I make towards it gives me a great sense of fulfillment, even though sole perfection is a very distant goal. If satisfaction is gained only by attaining the final aim, then probably no one would practice yoga because it is a very distant goal. Thank God, yoga is a process that gives satisfaction. So,

1 "I am very active in the class I give. Does that mean I am not meditating? You may meditate sitting in the corner, but I am moving everywhere and I am meditating. What is the difference between the two? Sitting in a corner and closing the eyes is not necessarily meditation - it may be just emptiness" (Iyengar 2002b: 162). 
for me, yoga is the most important thing in life; the deeper I engage in it, the more I feel the need to practice it. And, of course, there are many aspects that also move away from yoga. Living in the modern world I am attacked by such a great number of different stimuli that it makes my concentration spread. But yoga is undeniably the thing that is the most important in my life. (practitioner for 14 years)

Yoga as a form of meditation allows the practitioner to feel the whole body and connect the body with the psyche, and this accords with the formal definitions of yoga. Extremely important here is the notion of connection and integrity, just as yoga gurus define it. This definition is then very concurrent with the formal and original definitions of yoga. It confirms the knowledge of yoga philosophy in the case of some practitioners.

Because? Yoga, just like meditation, gives this rest to the mind in this sense that, err... that when you exercise, you do not think about these everyday matters, that there is something to be done, some problems, and so on and so forth, but you just think that your left arm is in such a position, leg in such a position, and at the same time you embrace with your whole consciousness your whole body, right? It is not like that when during the day we function, you do something, for example, with one hand and in your consciousness exists the movement of this one hand, but you work as a whole, with your whole body. And in this moment your attention exists as a whole... (moment of pause) I don't know if I'm saying this right... err, just as if you compare what you have, you focus on your leftright hand, first you feel this movement, left and then right, and then you try to feel both at the same time. And in the same way this integration that is in yoga focuses on feeling the whole body and the same thing is true with meditation. (practitioner for 5 years)

Again, the language used in the texts of the main yoga masters is absorbed by teachers and practitioners. B. K. S. Iyengar also often writes about "linking", and emphasizes that the word yoga derives from the roots yuj which means linking, bonding: "Yoga means union. The union of the individual soul with the Universal spirit is yoga. But it is too abstract a notion to be easily understood, so for our level of understanding I say that yoga is the union of body with the mind and of mind with the soul" (2002b: 3 ). "To practice yoga is thus to unite the body with the mind. For the cultured person, it is also to unite the mind with the intelligence and for the still more highly cultured person it is to unite the body, mind, and the intelligence with the depth of the soul" (Iyengar 2002b: 5).

This knowledge about the linking of the body and the mind in yoga practice can also be obtained during yoga classes, when the instructor says: 
I was in a free class "Yoga in the park." It is a project promoting yoga in the whole world. Classes in the open air, classes ran by A. K. Before class she gave leaflets about her studio "Anahata." During the class she told different stories about yoga in order to encourage the participants and explain to them the meaning of yoga. One of her comments was: "Yoga influences our bodies and next our mind because the mind and the body are one, and it is about integrating them. We do not set goals in yoga because after gaining one aim, there is another one. In yoga, it is the direction that is important and gaining it. Yoga is not sport, not a competition." (participant observation, August 24, 2008)

Sometimes it is visible how the commonsense meanings of yoga and practice are worked out during the interaction. This reflects the social development of common definitions of yoga. It is especially visible in discussions and exchanges of beliefs that take place with yoga teachers. Below is my conversation with a yoga teacher and one of the practitioners:

At one point a chiromancer left the locker room and the conversation was dropped after he stated: "What philosophical disputes?" Moment of silence.

I stated that we, in Europe, have a competitive attitude, that we also come up with our own ways to relax and develop, which is through sport. But there the ego is always engaged. The master stated that ego disturbs and interferes. I stated that the East has a completely different attitude to life. Chiromancer asked: "Then what, material things are not important?" The master stated that it is possible to have a practical approach to life, but it is necessary at the same time to have distance to everything. I say that after yoga practice it is often difficult for me to get back to reality. He says one can normally function, but with that distance. And I say that yes, that I am relaxed and I do not get upset with the workers, that I let it go... Chiromancer said that 2012 will be the end of the world and there will be a lot of vibrations and shakes, a lot of energy in the sense that everything ends with a moment of silence. And he distanced himself from this after a moment, saying that in 2002 the world was supposed to end and it did not... Chiromancer left, and after a moment I left too. (5 months of practice of the author of this work [participant observation, January 26, 2008])

\subsection{Conclusions}

Yoga practitioners try to individually define what yoga is for them. In general, these definitions are connected with the influence of yoga firstly on the body, and then on the mind. The role of yoga practice in calming the mind and emotions is underscored. This therapeutic part is also valid in the common definition of yoga. Yoga, according to practitioners, is a very individual action (metaphor of "a lonely trip"), where the practitioner examines him/herself and his/her capabilities; in order to understand yoga you need to practice it. The most advanced 
and motivated participants in the world of yoga try to achieve a fuller understanding of the spiritual aspects of yoga. The concept of "meditation in movement" appears here. Advanced practitioners talk about what real yoga is.

All of these elements are well-described in the books of the yoga masters, especially by B. K. S. Iyengar. His language and descriptions of experiences with the body and the psyche are reflected in the stories of those I researched. Of course, they do not always quote Iyengar - sometimes they do not even know of his existence, and often they do not know his works. Nevertheless some part of those researched know Iyengar's works, especially the advanced participants of this social world. The language of description comes most often from classes when instructors call certain emotions in a specific way, often after class revealing their interpretations of yoga in conversations, which are assimilated by the practicing adepts of the hatha-yoga world. Teachers usually know the books of the masters. The commonsense definitions, however, while reached individually and strongly concentrated on the individual needs and dependent on the motives of the yoga practitioner, are nevertheless inspired by the yoga masters and their works. This commonsense knowledge is some form of processing of knowledge attained fragmentarily, and not systematically from yoga teachers, as well as knowledge acquired from individual readings and studies on yoga. The freedom of the individual to define yoga is apparent here, because it is limited by the lexical resources and interpretations taken from above-mentioned sources of knowledge. The individual, being in the surroundings of teachers and other practitioners, learns this knowledge by processing it and filtering it at the same time through the net of one's own motives and needs. The individual constructs these needs and motives on the basis of information obtained from the media and other sources of knowledge existing in the modern world (e.g., the Internet). The individual creation of yoga definitions thus has social dimensions and inspirations. "The lonely trip" is done with the support of the social world of hatha-yoga.

However, something still remains undefined in yoga practice. There is a certain secret existing that is not possible to be passed on. The yoga adepts also underscore this when they say you need to practice yoga in order to understand it. The practice takes place with the help of the body and meditation. This is not a practice with a reflective character and rationale, and capable of being explained fully by language categories. This esoteric traces of yoga practice are still visible in today's yoga and its definitions. Hence yoga practice does not depend on language constructs or interpretations, but on real work on the body and psyche, and often on a spiritual search. 



\title{
CHAPTER 4 \\ The process of becoming a hatha-yoga practitioner
}

\begin{abstract}
It needs to be remembered that the body is the temple of the soul and it needs to be nourished, cared for as if making the place saintly, then it is in harmony with our mind... Yoga teaches how to love your body, it is a great gift, we got it on the day of birth and it is a great gift and we should take care of it, love it just the way it is.
\end{abstract}

(from the interview with a yoga instructor)

\subsection{Introduction}

This chapter ${ }^{1}$ undertakes the problem of perceiving and feeling the body in the process of acquiring the identity of hatha-yoga practitioner. The process of becoming a "yogi" is connected with the practice of work on the body and on defining these practices, engendering a specific perception of the body, and feeling the body.

Becoming a hatha-yoga practitioner is a process. In this chapter I describe the phases of this process: 1 ) the initial phase - constructing motives and first steps; 2) the phase of a fuller recognition of psychophysical effects and ascribing appropriate meanings to them; 3 ) the phase of a fuller recognition of spiritual aspects of hatha-yoga (as a quasi-religion). The relations between the mind and the body become complicated at the moment of meaningful engagement in yoga practice and in defining body practice as mental practice, as well as spiritual. The work on the body can change the Western perspective of defining the body as a material element of human existence (the Cartesian vision), to a vision of treating the body as a spiritualized substance (the

1 The chapter at hand is an elaboration of the article „Ciało świątynią duszy, czyli o procesie budowania tożsamości praktykującego hatha-jogę. Konstruowanie prywatnej quasi-religii" [Body as a temple of the soul - the process of building the identity of a hatha-yoga practitioner. Constructing a private quasi-religion] (Konecki 2012). The reprint of the extract was done with the consent of the editorial staff of Przeglad Socjologii Jakościowej. 
vision of Eastern philosophy). Such a change is not always possible if we hold on to the guidelines of other religions as our own (e.g., the Catholic religion). In such a situation, changes in the body and psyche have to be defined differently and also certain language descriptions of these changes have to take place (often acquiring guidelines of set languages formulas) in order to combine the statements of conventional religion with the new spiritual experience.

Gathering the materials for this chapter was mainly based on analysis of the results of the unstructured and narrative interviews, mainly with hatha-yoga practitioners, although there were also interviews done for comparative purposes with people practicing yoga-ashtanga. These interviews form the basis for the analysis of the process of becoming a yoga practitioner, although participant observation was also used.

In many interviews during my studies yoga practitioners have mentioned the difficulties in passing on knowledge about yoga and its practice (see: Konecki 2010). The interlocutors sometimes showed asanas and/or breathing in order to bring yoga practice closer to the researchers. They usually encouraged the researchers to do yoga so that they could understand it:

To tell the truth, not all of this can be told and explained, at least it is difficult for me to pass it on to somebody who has nothing in common with it. You just simply have to go and try it, see it, live it. (practitioner for 2 years)

It is very cleansing, relaxing, refreshing... so, so... well, I cannot describe it myself, you need to try it yourself, and you will admit I am right. I am serious. To be honest, everyone that I know when they started practicing they did not give up. Because you get into the swing of it. (practitioner for 2 years)

At some point, there comes into being an embodied knowledge and the practitioners forget about the rules and posture descriptions. Embodied knowledge substitutes the conceptual knowledge (Pagis 2010a: 487), and intersubjectivity can sometimes be created without words (Pagis 2010b: 322). Even though the verbalization of the experience of yoga practice is difficult, the interviews gave the researcher a lot of information about the motives and the stages of the development of the practice, etc. The interviews also made the practitioners reflect. Some of them said that thanks to the interviews they were able to rethink their practice, its effects, and their motives for doing it:

If I can (laughter) add, these are like my thoughts. And, I would even like to say thank you for this interview because it made me think. It made me wonder. Because I started doing yoga without having any idea of it. (practitioner for 4 years, age 46) 
The problem of auto-definition of yoga practitioners is connected with the ability to perform asanas. The one who knows how to do asanas and ascribes them specific meanings is a yoga practitioner. The practitioner reaches this state through practicing asanas and also studying yoga and listening to teachers' comments, sometimes performing breathing exercises (pranayama) and meditation (jnana).

\subsection{Introductory phase - construction of motives and first steps}

The introductory phase consists of three substages: a) constructing motives; b) first steps - the beginning of the practice; c) perceiving the effects.

\subsubsection{Constructing motives}

Some people believe that the decision to take up yoga was intuitive. They simply knew for themselves that they had to do yoga. Hence, for this group of people there were no motives that they had to become aware of before starting the practice.

So, my adventure with yoga started when I was 17 years old, and it was an intuitive decision. I knew nothing about it, I did not read about it, I just felt that I should attend yoga classes without even knowing what it was. I was just walking down Politechniki Avenue and I saw an invitational poster to such a class and just like that, dressed in a t-shirt and jeans, I went in and stayed 'til today. It has been years since that day. (yoga instructor)

It may be said here that, apart from intuition, the poster advertizing yoga classes played a major role in making the decision.

One can decide to start practicing yoga because it is popular or in fashion, which is noticeable among friends:

I have been doing yoga for a year now. To be honest, I took it up because of its popularity; one can even say that it is in fashion now. In fact I had no special health issues, while a lot of people take up yoga because, as I know, they want to stretch, etc. ... A lot of my friends started practicing yoga, and they kept on talking about it. I also wanted to be able to say something about it, be able to say that I do it too, that I know how it is. (practitioner for a year)

It needs to be underlined that the motives are often constructed ad hoc and in situ. Some experienced practitioners with, for example, ten years of practice, do not remember their motives at the beginning when they started yoga practice: 
- Can you tell me where your interest in yoga comes from? How did it happen that you started practicing?

- Well, it started... well, just like that. It just got me... the interest; health maybe. (man, practitioner for 10 years, age 51)

Sometimes practicing yoga starts in a difficult moment in the life of an individual. The individual may hope that through yoga s/he will solve his/her problems. In the interview cited below the exact nature of the problems are not stated, however, from further parts of the interview we find out that the interlocutor was emotionally unstable:

And... and... I started practicing in such a moment of life where I started this huge work on myself and on my character, my life. I raised myself up from such a ditch and it gave me so much, really. I mean, it was not the only reason that helped so much, but... er... er... it really brought a lot into my life, that is why I felt that... that I have to continue it, and now it is a natural part of my life, right? Although, I still feel that it's not enough because this everyday life disturbs me. (practitioner for 5 years)

The above resembles the situation when persons going through some life troubles join sects. It is often emphasized that joining a sect may be the result of some drama and personal problems of an individual. For example, people leaving prison can come across sect members who are supposed to help them (see: Wilson 1992: 184-185). What's more, many people can get through family problems because the sect offers them a new view on the world and allows them to regain selftrust (see: ibid.: 186). Obviously the sect offers emotional support and friendly relations, shows how unimportant one's current social status is, and shows the "ultimate truth," etc. (see: ibid.: 186-187). The sect members learn from one another which shapes their motives for participation and/or the ways to express them. The descriptions come from group participation. Remembering the moment of conversion is connected with language learning, which helps to talk about the conversion (see: ibid.: 200). Having said the above does not mean that I consider yoga practitioners as members of some sect; however some psychological dynamics and the participants' motivation in such communities, institutions, and practices can at times be similar.

Most often, the motives are reconstructed during the interviews and passed on to the researchers by the practitioners. The motives for practicing yoga often indicate that, by comparing yoga with other forms of physical exercise, they are somehow worked out in the process of "comparing justifications," as for example when one compares yoga with aerobics and points out the advantages of the former (the aesthetics of the exercises in yoga). There appears the presence of 
some kind of arena that singles out yoga from other the physical exercises (i.e., commonsense theorization on an individual level).

The motives for yoga practice can be different and may coexist with each other at one and the same time. With exercise and practice, new motives appear, which justify the sense of yoga practice (e.g., that it is a form of mental training). The motives thus show up not only at the very beginning of practicing yoga, but also later during different moments of doing or practicing yoga. The development of motives has thus a processual character. The initial motives for practicing yoga usually are:

- body training (stretching the muscles and/or strengthening them, body relaxation, treating yoga as sport, keeping fit, getting slimmer, maintaining slimness, flexibility and nimbleness);

- health effects (the aim being to improve the state of one's health);

- aesthetics, harmony of exercises;

- identity motives (yoga is treated as a niche sport or an alternative source of entertainment, with the participants looking for some occupation that singles them out from the surroundings, i.e. it is a singling-out-from-others way of spending free time).

The later motives, which show up over time together with practicing yoga, include:

- visible physical benefits, as well as health effects of yoga (visible improvement of one's health condition or curing different ailments);

- the peace of body and mind (quieting of the mind);

- positive influence on the psyche/mind - mental training (type of meditation, acquiring distance to the world, gaining a new perspective and way of viewing one's social surroundings, getting energy, etc.).

The above-mentioned body training (work on the body) is a category encompassing the entire set of motives that are mentioned most often in unstructured interviews and narrations. In the interview quoted below, the interlocutor mentions many of the above-listed types of motives. Reconstructed during the interview, the different motives can be thus presented by the same person at the same time:

- And what motivated you to start yoga practice?

- Yoga always attracted me. First, it attracted me as a form of gymnastics, I think most appropriate for me because I do not like to tire myself too much, I hate running, aerobics and power training, strictly for fitness when you sweat streams, cannot catch a breath, and the heart jumps out through the throat. This is not for me. I am not used to such an ordeal, painful bodywork or these, er, types of activities. But yoga includes such exercises that right from the start are very nice, aesthetic, feminine; and if you know how to do them well, they are very harmonious and calm. They do not set the tempo to start with, or the number of repetitions, and while it 
a time is most often specified during which a given asana should be done, there are no stiff rules that you have to keep to and that you cannot free yourself from. The human is in asana as long as the physical capabilities allow him to be and as long as he feels comfortable at the mental and physical levels. At first, yoga interested me as a form of physical exercises, training only for the body, and then I started seeing in it a form of mental training, a type of meditation, a kind of a calming remedy, acquiring distance to all of that which surrounds me. And well, the physical benefits are also important because through its specific interaction yoga has a lot of health properties. (no data about the years of practice)

Yoga's positive influence on the psyche is sometimes the subject of additional elaboration. The below quoted yoga practitioner asserts that she was not subjected to anybody's suggestions and that nobody suggested to her earlier the positive influence of yoga on the emotions and the psyche. She claims that yoga specialists would agree. Nevertheless, it is difficult to believe such claims in their entirety, since earlier in the interview she talked about some books and about possessing of some (according to me, a priori) knowledge about yoga and its influence on the body and human psyche. This previous knowledge could have had an influence on her perception of what happened with her psyche and body as an alleged consequence of doing yoga.

Apart from that, I have noticed that it has a positive influence on my mood. Because, frankly speaking, I was quite skeptical at the very beginning. Well, I did hear before that yoga has a positive influence on emotions, so I did not want it to influence me... But, I think that in my case it was not a case of suggestion here and it just came to me and I really felt at one point such a flow of good, erm, positive emotions. This influence on the mental state surely would be acknowledged by some yoga specialists... (practitioner for 5 years)

For some yoga practitioners it is only gymnastics, and they approach the information about the spiritual aspects of yoga that they receive very skeptically:

I am talking about an inner peace, but these are only things that I read somewhere, uh... I did not meet with such yogis, or such type, you know, some film you watched, or such and these are some foggy ideas, you know, you hear it somewhere, it rings a bell, but you are not sure. So what values does it have for me at this point? None, apart from gymnastics. (beginner in yoga practice, age 50)

The motives for practicing yoga change over time. In the case of more advanced "yoga practice," it often becomes a value, a very important element of everyday life and thus the motive for yoga practice is this value, which determines the life of the individual: 
Anyway, I am not going to give up yoga. At this moment, I cannot imagine life without it, it has become something very important for me. Thanks to yoga I can disconnect from the problems, it somehow allows me to approach everything calmly. (practitioner for 3 years)

If yoga becomes a value for practitioners, then they use descriptions differentiating their practice from other forms of physical activity, sport, or psycho-spiritual practice. This distinction between what they practice and such other actions is a necessary procedure in the work on the identity of a yoga practitioner. Features distinguishing yoga from sport or fitness work on the emotional and cognitive part and consciousness of the individual. Distinguishing (here I refer to identity motives) builds up motivation for the further practice of yoga as it makes this practice a unique action, rarely met when an individual builds, basing on the action, the concept of the I:

Somebody who thinks that yoga is only sitting and murmuring under the nose is very wrong. Ashtanga actually contradicts this idea because it is a very dynamic yoga where something is happening all the time, where you move smoothly all the time from one asana into another, you sweat a lot, the organism gets rid of all the toxins then... It is not just coming, exercising, like fitness just to sweat, but this is a more conscious learning of your own body. Yoga starts from this, and then you enter deeper into the emotional sphere, of your own deep consciousness. (practitioner for 2.5 years)

For some, yoga is only a set of physical exercises to which no values are ascribed apart from the possibility to stretch the muscles and relax. These people look for some kind of "sport" that would meet their needs. They often use the word "training," which suggests the association of yoga practice with sport. For them it is a form of gymnastics, sometimes treated as a substitute for correctional gymnastics. Some of them also do aerobics and aqua aerobics at the same time. Sometimes it is needed by some people to strengthen their exercise regime and/ or body while practicing other sports, for example, swimming, skiing, basketball, climbing, etc.

...because yoga is divided into two such, two kinds: yoga of the body - hatha-yoga - as it is called, which is, in other words, a form of common gymnastics and meditational yoga - something completely different. I was not interested in that meditational yoga, you know, being some flower or in reincarnation as I do not believe that much. However, I was interested in yoga as gymnastics and I saw after these three years that I am more fit now than I was 4 or 5 years ago. (beginner in yoga practice, age 50) 
Because, you know, it gives nothing but benefits, both physical and mental; I became so flexible, which is a plus while playing basketball. Endurance is also practice and this is useful always and everywhere... Try to hold on to a posture for 7 minutes, for example. (practitioner for 1 year)

Why did I become interested in yoga? The book and the other... these were just the results of this, showing that I was looking for something. And I did look for something because I wanted to develop in rock climbing. Already such strength training or some endurance training... well, it was not so useful. These effects were slow and I decided that maybe something else, something that would improve concentration or flexibility, would be better. And in the beginning this is how I treated yoga. As a completion of rock climbing. (practitioner for 14 years, hatha-yoga instructor $)^{2}$

For this type of practical motivation of sportspeople who practice yoga, Iyengar's descriptions of particular asanas are helpful. For example, for adho-mukha-svanasana ("downward facing dog") he gives such a description: "The pose is especially good for runners who get tired after a hard race. Sprinters will develop speed and lightness in the legs" (2005a: 35).

There can also be a situation where a person practicing yoga with the aim of improving his/her performance in another sport (in order to boost the body efficiency) changes his or her basic activity from a given sport to yoga practice. Yoga practitioners perceiving various effects of yoga practice may give up sport (e.g., rock climbing) and focus only on yoga practice. The motive of "yoga as a form of gymnastics" may be only an initial motivation for taking up yoga, and lead to other interpretations and actions of a given person in the social world of yoga practice:

But, as time passed, it turned out that this is not just some exercise that makes... us more flexible or that our concentration improves. We can spread our strength more effectively and more effectively use what the body can do. It turned out that I feel better, yes. I started getting more than I expected it would bring. And while through these same years I climbed, I started climbing less and spent more time with yoga... And, after 3 years, encouraged by Sławek, I felt that I know so much that I should share this knowledge with other people. He said that I should start teaching and coming to him, so I could learn how to teach. Because it is something completely different to practice for ourselves, and something else when we teach others. And then it intensified even more... with my going to Warsaw. This lasted for another year. Finally, I started teaching. (practitioner for 14 years, hatha-yoga instructor)

${ }^{2}$ See also the interview conducted by Bartosz Dobroch (2008) with Steph Davis, champion of rock climbing, who assisted her practice with yoga. 


\subsubsection{First steps - beginning of the practice}

The "first steps" stage is connected with those actions that an individual undertakes in order to start yoga practice, or other actions that one undertakes and experiences at the very beginning of contact with the world of yoga.

Many practitioners point out that before taking up yoga they had heard a lot about it and/or read about it. The Internet is a very important source of information. Some practitioners have even said that if it were not for the Internet, their yoga practice would have never happened. What's more, stretching exercises were their favorite ones, which is why they ended up in a yoga class, or after the initial introduction to yoga started practicing it regularly. Some of them also mention an interest in Eastern philosophy, for example Buddhism, and do not necessarily point to an interest in yoga itself. It is, in general, about interest in the spirituality of the East and meditation. In the case of many people, the need for meditation appears in their future. Some of them mention Buddhism as a subject they were also interested in. So in constructing their spiritual interests the researched persons showed a syncretic attitude. In Great Britain, many of those regularly practicing yoga identify themselves with Buddhism (Hasselle-Newcombe 2005: 315).

You can get to yoga classes also through P.E. during studying, when yoga is sometimes one of the types of physical exercises you can choose. With yoga exercises we also meet at fitness class, which can inspire a deeper interest in yoga.

Often, certain schools of yoga were chosen because they were suggested by friends, acquaintances, colleagues, partners, brothers, sisters, and even parents, as well as under the influence of advertisements. For many, attending yoga classes with friends is more motivating than individual attendance, without support from one's own social circle. The recommendations and suggestions of yoga practice from people coming directly from one's social circle are seen as particularly important:

- Please tell me, how did you become interested in yoga?

- Half a year ago my sister had a difficult time in her life, she got divorced, in general, that was a difficult experience. She could not cope with that... To put her life back together somehow, I tried to help her somehow, but she was not so keen on being with other people. And some time passed, and one day she phoned, invited me to her place and she had such a different voice, so normal... erm... so calm... I was really surprised by that and very curious at the same time what changed her because it was so strange... and we met and she told me that she found her balance and that there is no point stressing yourself out so much and worrying about 
everything. I saw how she said that and the way she was in general, how she behaved, and I could not believe it, and after what she had gone through. I asked her how she did it and it turned out that she had started doing yoga... I did not want to believe in that because you need time to achieve results, but she told me that she exercises a lot, that she attends professional yoga classes and that she exercises alone at home, she said that she tries to exercise in any free moment, and that she has no time to worry and sulk... And also that she can sleep normally because she is tired after all this effort. So it was my sister who inspired me, I will be honest, that I uh... did not know anything about yoga, it was for me... so... I don't know, so illogical, I always thought that this is some trend, that this is all advertisement, it is difficult to talk about this just like that if you have not tried it yourself... (exercising for half a year, age 40 , secondary education)

Starting to practice yoga can also be connected with the inspiration coming from friends. The direct influence of a circle of friends circle appears to be very strong. Such an inspiration can cause one to search for different information about yoga:

It started from with my friend from a group was doing yoga and one day we started talking about it, she started telling me about it, what it is about and, in general she was praising it in every possible way (laughter). Well I felt a bit, you know, as if she was trying to sell me something (laughter). Anyway, she made me a little curious, so I searched the Internet about yoga, and, I found some websites of schools. I read about it in general, what it looks like, I found some articles, there were also some, erm, self-study guides, such ABC yoga, you know, so you could exercise on your own, but I don't know... Somehow I didn't undertake any decision to start practicing yoga, it ended at reading and I also watched some films. (narrative interview with yoga practitioner for 3 years, age 22)

It is very important for the majority of practitioners to acquaint oneself with books about yoga, and often these are various materials available on the Internet. Some, while giving the reasons for beginning the practice of yoga even reconstructed, in narrative interviews, their interest in gymnastics when they were children, when they liked, for example, to put their leg above the head. They ascribe their interest in yoga to these exercises and interests.

The first class can be a shock to beginners. Sometimes it is extremely difficult to do the simplest asanas because the body is not stretched. Going through this class can either be encouraged to further practice, or discouraged from it. Pain appears:

At the beginning, it hurts a lot, it means the body is not fully adjusted to such an effort, well, you know how it is when you stretch and you feel such pain, because you cannot do more, you feel such a tension. But, this passes with time; the longer you practice, the more your body changes, becomes more flexible, more stretched (practitioner for 7 years). 
The motivation to practice yoga is already built into the initial phase of practice. This refers to the perception of the corporal effects of yoga practice. Some call it "crossing the borders" of the body that are placed before the practitioners by their own bodies (the body resists). It is important to correctly define the pain that appears in this phase (as pleasant pain).

It was great for me at the beginning, such crossing of borders of my own body. During the class I felt such muscles that I did not even know that I had (laughter). With each class I really felt how everything stretched. Although, I have to admit that in the beginning it was not easy, it really hurt and I twisted my face in all ways (laughter). But, with time the pain disappeared, or maybe I got used to it. Anyway, it was in the end all such a pleasant pain... (no data about the years of practice)

Often practicing at home I tried to remember the teacher's instructions from the class at yoga school which helped me to properly define my reactions and the feelings of my body: "Feel how nice stretching is. Is this a pain from stretching? If yes, then it is nice, feel the pleasure from stretching... If your back hurts, that means that you are cleansing yourself, all these unnecessarily accumulated burdens are cleansed..." (participant observation, auto-ethnography, August 16, 2012)

The concept of 'pleasant pain' is often used in the interviews; pain is a sign of cleansing the body and psyche. After cleansing there is an improvement in the mood:

And when I walked, it really hurt in general after these classes because it was a lot and intensive. But, this is a pleasant pain and cleansing... During exercises I simply feel good, well, because I get tired and it hurts me, but this is such a really pleasant pain and tiredness, that the person later feels so light, delicate, healthy, and alive. (practitioner for 20 years, with breaks)

The concept of "pleasant pain" appears in B. K. S. Iyengar. Feeling the pain and crossing it has a spiritual character and shapes the spiritual posture in yoga, even if the practitioners do not know that or even if they deny it ${ }^{3}$ :

Only when there is a pain will you see the light. Pain is your guru. As we experience the pleasures happily, we must also learn not to lose our happiness when the pain comes. As we see good in pleasure, we should learn to see good in pain. Learn to find comfort even in discomfort. We must not try to run from the pain but to move through and beyond it. This is the cultivation of tenacity and perseverance, which is a spiritual attitude toward yoga. This is also a spiritual attitude toward life. (Iyengar 2005b: 47-48)

\footnotetext{
${ }^{3}$ See also paragraph 4.3 .
} 
What Iyengar writes does not have to be known to the practitioners, and they may reach this knowledge by practice according to the suggestions of yoga instructors. Not many of those practicing hatha-yoga read and/or remember the writings or lectures of Iyengar.

So you can feel already after the first class. I felt an immense flow of energy after such a first weekend meeting with Sławek. I did not want to believe what an influence it has. These were really difficult classes involving endurance, such that after each sit, I was exhausted... and I left as if I was walking after class on tilts, floating above the ground: light, so happy, I had a will to just run. In my case, this was almost from the first class, from my first contact with yoga. (practitioner for 14 years, yoga instructor, owner of a hatha-yoga school)

The body integrated with the mind often resists, especially with the beginners. This resistance needs to be overpowered in order to develop further. Below is my own impression from my first classes:

Today, I felt since the morning like in a cocoon. I did not want to open to the world in the morning because of yoga. I wanted to stay focused, almost half asleep, so the outer world would not reach me. I went for a walk. This feeling persisted. It was about 17.00 when yoga exercises allowed me to open myself, stretch my body and at the same time allow the perception of the world to reach me and accept it the way it is. I could be with the outer world on equal rights without the protection of my inner world, and at the same time together with it. I cracked this cocoon by stretching myself and staying in the positions for a long time.

It is a strange feeling, wanting to be in a cocoon. I would not know that I was in it if it was not for yoga. This tendency is something so natural, it is a natural wholeness that is difficult to notice. It is an everyday normality of the body, sometimes stronger, sometimes less strong. Physical exercises break it. They allow the body to reach normality with others, while being itself at the same time.

Every day I notice that stretching creates space for me, gives more space in the body and also in the mind. This is my interpretation based on what I heard once from master Szczepanik. He said then, during exercises: "People move out outside the city to the countryside. What do they want? They want space. We also want more space." Master gives me more ways to understand what happens to us during exercises. Maybe without these interpretations we would not see what I am writing about now. (5 months of exercises of the author of this work, participant observation and autoethnography, January 30, 2008).

First classes are extremely important to undertaking further efforts in yoga practice. Going into raptures over it, marveling at the exercises, can be a very important element of building the motivation for exercising:

That is why yoga seemed to be the best answer to my needs. And all that I thought about yoga, what I imagined, was confirmed during the first class. I discovered at 
once that yoga is a type of physical activity that is made for me (laughter). I left the first class simply marveled and I decided at once that I needed to sign up for yoga somewhere, some extra classes, because just once a week was simply not enough for me... (practitioner for 5 years)

...then, the classes were run by Michał Szczepanik and I found myself there. And as the classes were run I felt suddenly as if it was mine, like from the bottom of my heart. It was easy for me to do these exercises and then I had no awareness of people who are there, had been there already several years and they knew this whole cycle and some Sanskrit names. I did not know about that yet so I was such a, you can say, a person that knowing nothing found herself in such a place that was later like such a seed that starts growing. (certified teacher of yoga by B. K. S. Iyengar's method)

Some people start their adventure with yoga at home, doing it on their own using guides available in a book, although it can be dangerous for their bodies without any control of an instructor:

I started with learning yoga at home (laughter), I borrowed a book, I think it was Yoga at home, and this is how I started. Everything was described there, step by step, first, the preparation to the exercises, learning how to relax, breathing, looking after flexibility of the body, etc. ... When I told my friends that I would also be doing that and that I started with this book, they discouraged me. Generally, I read on the Internet about this too, that it is better not to start on your own, that you can get injured, that you can discourage yourself very quickly and in addition it would be difficult to exercise regularly... But, I wanted to try myself first. And, frankly speaking, there was a lot of truth in what everybody was saying. (practitioner for a year)

Entering yoga practice is not an one-time decision action, it is a process and takes time. As we can see, it starts with the interest in yoga by acquiring information about it and it may happen then that this interest is put to sleep and during some situation, for example, a promotional event (this would be an intervening condition), the decision about taking up yoga is made.

Well, if I may say so, the thing just hushed (laughter), sometime later I was on Piotrkowska Street with a friend, I don't remember why, probably to gossip, because at the end of high school we rarely met, she had her high-school final exams, I had my high-school final exams, so we didn't have time... But back to the point, so we got a leaflet from some woman that she organizes yoga classes, and that was a new school, and for promotional reasons you could come to first class for free. This is true, and it was such a fluke that I read it because usually I do not take such leaflets and even if I do, then, you know, I throw them in the nearest bin... But, I looked at it and I started convincing Anka to go with me and see, that it was for free, so you can't miss it, and she finally agreed, although I was more zealous (laughter) and well, and if I insist on something, I always get it, she knows that. So, she finally agreed so I would give her peace (laughter). So the following week we went to this class, me with a very positive attitude, Anka complaining a bit that it is not for her, and so on... (narrative interview with yoga practitioner for 3 years, age 22) 
One of the aims/motives for doing yoga can be the desire to obtain awareness of one's own body (focusing on the reactions of your own body). At the same time, through being aware of your own body we can focus on the here and now and disconnect from thinking, overbear the psyche:

I attended classes because I was interested in one aspect of yoga that I had read about somewhere, something that was at the beginning rather abstract for me, which is the awareness of your own body. It is difficult to describe. With each next posture we discover our body more and more, we learn about it, we try to listen to it. It is important to focus on the reactions of the body. For this to truly work, you know, you need to be really focused, try to think only about what you are doing at a given moment... And it is known that this is not easy at all. All the time, the head is filled with different thoughts and it is difficult to disconnect from it and focus. (practitioner for 3 years)

Teaching the awareness of your own body is also connected with the reaction of the body in specific life situations, for example, during pregnancy:

I, after my own experience, believe that you can practice and the body will tell you what you can or cannot do; I can stand on my head, but I cannot do the candle because I feel sick, so in general I do those positions that I feel good in and when I feel that something is wrong, that, for example, I feel very hot or I start sweating a lot, or I don't know, I have some hot flashes in my head, then I stop doing such a position. Of course, there are in yoga some positions more and less recommended for pregnant women, for example, as I also read during pregnancy. (fitness and yoga instructor)

Observation of one's own body is the tool to achieve body awareness, although this motive appears a bit later, when the practitioner has gained more experience:

Yoga gives you this that you can observe your body, because a given position will be for one man very easy and for another very difficult, and vice versa. Everyone will find there some positions that are very easy and some that are undoable. Of course, there are different variants and this undoable position may be doable with various help and in a different variant. (fitness and yoga instructor)

Body observation is connected with knowing it and discovering its reactions, strengths and weaknesses, inhibitions and restrictions. Often one can observe emotions accompanying the exercises, for example, fear:

Practicing headstand I felt a bit scared. I have also noticed that very important is precision when doing the candle or standing upside down. Every ges- 
ture and sequence of movements in performing these positions are extremely important. It needs to be done as it should, which is how the teacher says. I have also noticed a paradox that in the candle the strength of legs is very important. Having your legs up it is good to have them strong so they would straighten up and pull the body up. It is easier then to keep in balance. ( 5 months of exercises of the author of this work, participating observation and autoethnography, January 26, 2008)

In the initial stage of the practice, one might change the teacher. The practitioner is looking for a teacher that is adjusted to his or her character and psyche. After finding the teacher there is stabilization in the practice:

Because when you start doing something, then suddenly it turns out that somebody else does something else, and suddenly it starts as if we are really open to something. So, it turned out that a friend of a friend was going and she started going and we understood each other, and then she went to a different teacher, so I thought maybe I would try some trip at the beginning... and this, when I went to the other teacher, then it went on and I am there for eight years now. And in the beginning, I attended the classes almost every day. Apart from some breaks this has been 'bull's eye', and until today yoga is for me just an obvious thing in my life. (practitioner for 8 years)

Motivation for yoga practice can be stopped if the beginner has too high expectations. If one wants immediate results, then one can have doubts after some time as to the sense of the practice, and skepticism can appear. Beginners may look for effects and defined feelings, including quick effects. They try to perceive them based on their prior expectations and possessed knowledge, and maybe the observation of celebrities who do yoga (e.g., Madonna). Madonna ascribes yoga the following properties: "Yoga is a metaphor for life. You have to take it really slowly. You can't rush. You can't skip to the next position. You find yourself in very humiliating situations, but you can't judge yourself. You just have to breathe, and let go. It is a workout for your mind, your body, and your soul." ${ }^{4}$ Madonna treats yoga very practically, as exercises for the body, mind, and soul. It is interesting that Madonna mentions the soul here, which shows the religious connotations of yoga in the system of values of the celebrity. Other celebrities doing yoga include: Sting, Jennifer Aniston, Gwyneth Paltrow, Ricky Martin, Meg Ryan, Drew Barrymore, Reese Witherspoon, Nicole Scherzinger, and Mathew McConaughey (Górski 2004). ${ }^{5}$

${ }^{4}$ See: http://magazine.foxnews.com/celebrity/hot-celebrity-bodies-shaped-yoga (accessed: 19.07.2014).

${ }^{5}$ See: http://comfortinstylemag.com/tag/yogis (accessed: 30.01.2011). 
If the beginner is not aware of the fact that yoga is a process and the effects come with time, then he or she can get discouraged from practicing it.

I expected that yoga would allow me this... uh... emotional education. Hmm... Yes, I think this is how it's called, that it would be easier for me to focus, that I would have more patience in general, that I would cope better with the stress and different tensions. Frankly speaking, I think I expected some miracles (laughter). Yes... and you cannot, really. With such beliefs you can easily discourage yourself, resign quickly because there will be no results. That is, I believe that for some yoga really gives them some strength, that it changes them, transports them to as if a different state of mind, but I do not feel it. Maybe I've practiced yoga for too short a span... Definitely, without any doubt, it is so that something is improving, but anyone who does any sport can say that, after all there are hormones secreted so that the mood changes into a positive one, that is what yoga gives me, a good mood - not a special one but a normal one. Thanks to these classes I ensured myself in the belief that yoga is it, I feel my muscles, I feel generally better, and I hope that there will be bigger effects, that I will feel it somehow because I would like to spring with energy a lot and be calm in difficult situations. That is what I would like the most. Yoga has calmed me down a bit, but as I've already mentioned, it is more slow than the effects that others have. I do not know whether, if I practiced yoga for example for 10 years and there were no such effects, that I would want to still do it. It is not so that I link my career with yoga, because one can hear about this more recently, but I would want it to considerably change my life. I hope that I will not need 10 years (laughter)... (practitioner for a year, age 22)

And at the very beginning I was very disappointed because I do not know if you remember, but Madonna is a big fan of yoga and when I read about her and watched her video clips and she is so flexible there, then I thought that oh, that's cool and I will do it too. You know, such a simplification sprang into my head, and then nothing. In the beginning, they tell me to breath in a correct way. Ha-ha, my first impression was that it is all going to slow. I wanted it to be so quick. (practitioner for 4 years, age 23)

If the practitioner does not connect, for example, social aims with yoga practice, and only sticks to visible physical and mental effects, then the lack of expected and positive effects can result in drifting away from the practice. When a practitioner sees no effects, then what could keep them practicing in such a situation is a further attention to their expectations and keeping them under control.

\subsubsection{Noticing the effects}

To remain in the practice it is very important to perceive the effects of yoga; they build a strong motivation for further practice. This perception of effects takes place already in the first phase, and 
especially at the end of it. Recognition of the effects is different in different moments in time in the practice of yoga. Below Sławomir Bubicz (a student of B. K. S. Iyengar) and an expert in the processability in recognition of the effects of yoga practice, points out some stages:

When do the first effects of exercising appear? I ask Sławomir Bubicz, the director of Hatha-yoga Academy. Just after a month. Better sleep, better silhouette (you keep up straight, your walk is lighter), better blood supply (hands and feet stop getting cold), more peace in stressful situations, calmer breath. After half a year, the relations with people start changing. You are more emotionally stable, you do not get "infected" with the negative emotions from others. A feeling that everything is ok starts coming over you. You stop feeling regret - towards God, parents, anybody (Staszewski 2009).

One can risk the thesis that without any visible effects in hatha-yoga, practitioners move away, and that if they perceive the effects, they stay. It is also important to see the influence of yoga on everyday life and the decisions one undertakes (here I mean the effects outside the practice itself) and on their behavior. The researcher himself one day after five months of exercising - noticed the effects of practicing yoga; and today, looking at it from the perspective of the researcher, he ascribes instrumental meaning to yoga exercises.

After doing a cycle "the Moon salutation" I was straightened and charged all day. I straightened myself all the time, in the train, on a walk, entering Warsaw University to participate in a Ph.D. exam. Reading on the return trip in the train I experienced great efficiency. (5 months of exercises of the author of this work, participant observation and autoethnography, February 26, 2008)

\section{Other practitioners recall:}

When it comes to me, it is obvious that I myself had such difficult moments, when I was not entirely sure that my decision was right. Because, you know, what you do during the class you feel later inside, in a normal life, today, for example, I... after doing such a standard set of exercises, feel the difference since I started going and improved my mood. I noticed that I could do something else; yoga was for me like an impulse due to which we decided to do other things. I think a year ago I signed up at a swimming pool and I go to swim about three times a week. Having such a connection I feel how my body is working and above all my mind. (practitioner for 3 years)

Because I haven't said this yet, but yoga teaches one a different outlook on the world, a different perception of reality. This helps a lot because it allows you to change your perspective, see things that you have never thought about... I was always a very chaotic person, so unorganized, crazy... This always made my life difficult, you know... And, now thanks to yoga I have more time for everything, I can 
organize myself and manage to do all of the duties I have; and, additionally, I have plenty of time for myself. This may seem improbable, but it is true! Dang it; it is fun... (practitioner for 2 years)

Sometimes, after stopping yoga practice, practitioners come back to it after many years and again as it appears as it was in the beginning of the practice. An important part in these return engagements is played by friends:

I attended yoga when I was really young. Not for so long maybe, but it helped me a lot later on. Especially when I was pregnant and then when giving birth because I knew how to breathe better than normally. And, then I had a husband and children, and work. I stopped exercising. And when I turned 40 I started looking for something to do for myself. To keep fit and maybe move somehow... and... hmm... In the beginning, there was some meeting, I remember, with yogis, masters that my friends dragged me to so I would see, then, well, I heard all the time from them because they exercised - that it is healthy for the body and mind, that I will calm down, slow down, that I would feel better. (practitioner for 20 years, with breaks)

One's perception of health effects after some time of doing yoga are very important. The practitioners often talk about acquiring organism resistance and getting sick much less often. This also keeps these people practicing. Yoga instructors pay great attention to the health effects of yoga; the great propagator of hatha-yoga, B. K. S. Iyengar himself, underlined the curing properties of yoga ascribed to the healing properties of given asanas (see: 2005a), and in a different book, The Tree of Yoga (2002b: 37), he even mentions that he cured himself of tuberculosis. And in the book Light on Life he gives a set of asanas which create emotional stability (Iyengar 2005b: 267-268). In many training films, at the end of describing a given asana its specific healing properties are often underscored. The practitioners have then some preparation for telling others about such effects:

I don't have... I don't have muscle pains. Suddenly, it turns out that I am more physically fit when it comes to, for example, bending down, pick something up from the floor, nothing cracks, not that you bend down and 'ouch'! (pretending to be in pain), no. I don't claim that such things are gone forever because... because... because I don't know, but suddenly it is not only that I stopped this organism regress that had started, that I started seeing funny things, but I started seeing and some things just moved back. (beginner in yoga practice, age 50)

My body changed a lot. I, in general, have serious health problems, hips slipping out, this is such a defect from childhood, I do not have fully developed sockets so the hip bone comes out - I mean, used to come out in the childhood even very often, later less often... So, yoga brings about a high growth in flexibility of the joints and strengthens the muscles, for example, I do not know, but these that keep this joint 
in a good state, so there is no situation that I, for example, am standing and this hip suddenly comes out - it happened several times and was quite painful, so this is such a typical, one of many advantages of practicing yoga for me. I am simply healthier. (practitioner for 7 years)

In some cases, there is a kind of self-healing thanks to asanas practice:

- And do you recommend yoga training to others? What do you think, what kind of a person could find oneself in it?

- Any person, any person. There are tangible assets, fitness. I had problems with the spine so I helped myself, you can say, I set this spine, really, with yoga. I had a problem, like for three years I had this, but I managed and it is ok. (practitioner for 10 years, age 51)

What's more, yoga can also be done by ill and handicapped people:

Because yoga allows exercising when you are sick, when you have disabled limbs, some systems or movement apparatus disabled. There are always exercises in yoga that anyone can do; maybe not by a person completely paralyzed. (beginner in yoga practice, age 50)

The perception of positive health effects often causes some reflection on the condition and philosophy of the healthcare system in our cultural context:

... we have a bad system of health care. I believe that doctors should take their salary when the person is healthy and not sick. Because the doctor makes us dependent on the sickness and we make the doctor dependent on our sickness, which means it is good for the doctor when we are sick. In the East, it is so that the Ayurveda emphasizes health; that is, you pay the doctor there for being healthy, that he keeps us healthy, and we do not go there when we are sick and pay him for that... I now have many thoughts about this, that it would be good to change the system of health care when it comes to that aspect. To pay the doctor for keeping us healthy. Here, we call it more a family doctor, yes, we do not experience it completely. I, for example, did not experience a family doctor that has been functioning here for so many years. And it would be nice if we, as members of a family, even paid him some money for constantly looking after us, that we would stay healthy. And when we get sick, then he should pay us! (yoga instructor)

Health effects are often confirmed in conversations among the practitioners and also in conversations with instructors. If an instructor confirms the presence of healing effects of yoga, then this is a serious argument to accept such an interpretation of the way yoga works: 
Next, Michał talked a lot about the positive health effects for him resulting from doing yoga. He said that he would be walking with a cane if he did not do yoga. The doctor told him that he would have problems with his knee and it would stay like that. After many months of exercises, there is no sign of the injury. He also had his fingers twisted as if he had rheumatism, and yoga practice (downward facing dog) allowed him to straighten his fingers and today there is no sign after the endurance deformations caused by rock climbing. Similarly, he had problems with the spine. Today, he would be walking with a cane or be in a wheelchair, the doctors wanted to operate on him. Practicing yoga after a year and a half allowed him to get out of this problem. (9 months of practice of the author of this work, participant observation and autoethnography, July 11, 2008)

The first breakthrough moment and concrete effect of the work on the body in yoga practice can be the better physical feeling of the practitioner. In addition, he or she acquires the identity of a practitioner by perceiving the physical effects:

First, well, is that people feel physically better with their body. Taking care of the body is extremely important, and we here in this Western culture do not take care of the body like in the East. It needs to be remembered that the body is the temple of the soul, it needs to be cared for, nursed, cherished in this place, then it harmonizes with our mind. That is why you need to take care of it, you need to love your body; which is very important. Unfortunately, we Poles do not love our bodies, we constantly have something to criticize it about. If it is not so that we are too fat, then our breasts are not so, or we are not that tall, whatever, it is simply awful. Yoga teaches how to love your body and that it is a great gift, we got it when we were born and it is a great gift and we should take care of it, love it the way it is. (yoga instructor)

In the above quote the statement appears that "the body is the temple of the soul." We do not know if this is a conscious quote or not from the book of the hatha-yoga guru B. K. S. Iyengar The Tree of Yoga: "Because the body is the dwelling place of man, one should treat it as a temple of the soul" (2002b: 18, see also p. 3). Whatever the current situation, it can be said that some statements belong to the repertoire of the knowledge of practitioners, and some are reflections of symbolic surroundings which allow them to interpret what the practice itself and the perception of the effects of the practice are.

And I, myself, feel more fit, stretched more. This is a very nice feeling, you know? Earlier, before I started practicing yoga, I didn't care too much about fitness, and now it has become very important for me because thanks to it I can function better. (practitioner for 2 years)

A very essential element defining the identity of the practitioner is the moment of perceiving the effects of yoga practice. Apart from perceiving healing and psychic long-term effects, the practitioner also 
sees the effects right after finishing exercising (e.g., the effects of mental relaxation), during the last asana finishing every practice:

We end the exercises with a relaxation, this relaxation lasts even 15 minutes, and we lay down in a so-called corpse pose, well, then there are marvelous experiences, marvelous experiences. It is great. After this effort all of this comes back to being normal and the energy flows so wonderfully through the body, we can even fall asleep and sleep from all of this. Cool things happen at the end, such inner feelings; and the more the person is engaged, the better the experiences. Such a person is generally quiet, does not get nervous, has a lower voice, is calmer. Really, there is no hogwash here. Well, the benefits here are immense, I do not get sick. (practitioner, age 49)

A very interesting effect of practicing hatha-yoga is its influence on the social relations of the practitioner. Practicing yoga opens the body which, being in such a state, enlarges the openness of the subject towards other people. Work on the body becomes work on interpersonal relations:

- And when it comes to limitations that yoga eliminates, what does yoga give in terms of interpersonal contacts?

- Above all, a huge openness. The person is not even aware how the closing of the body is such a contracting of the world, how much it limits us. On one side, it limits us and on the other side, we close ourselves in our body do not want to go outside. And here, slowly, slowly the opening of the body causes the opening towards the surroundings. Really! (practitioner for 4 years, age 46)

A very interesting social phenomenon is the perception by others of the effects in a given practitioner. When the practitioner sees that others, often close persons, perceive the effects (a looking-glass self), it strengthens the motivation to practice and causes feelings of contentment and confirmation that one has made the right choice of the path. The subjective self of the practitioner is made stronger, and by the perceptions of others the individual pays more attention to new qualities of the psyche that were never there for him/her before. This is how the identity of a yoga practitioner is shaped to whichever new content is introduced:

I am definitely calmer, it brings such a great balance, mostly mental, but I suspect that it links with what we still develop physically. So harmoniousness in our body, it also brings about such mental harmony... very... I know that people at work also feel it, for they always ask me if this is the influence of yoga (laughter). And I say, I think so (laughter). So, it is a great, great advantage when others apart from me see some important changes in my behavior...Well yes, this is really true, yoga can give such a strength; my husband tells everyone that thanks to yoga I became a better partner in everyday life (laughter). (practitioner for 7 years) 
Well, the majority of people from my surrounding noticed that, as opposed to the majority of people my age, I sit up straight. For example, I know how to relax myself, I can rest quite fast, I know how to adjust my body to such a position that cause that I do not get tired when standing for a long time. I know how to relax my spine, leg, hand, that I am able to cope with some limitations of my physicality that result from my age, genetic tendencies, from such things, that is, physical limitations. I focus on that. (practitioner, age 53)

Now I see it all completely differently; I approach life differently. One such yoga therapy a week is enough and I surely have more patience. And even my friends or family see that I am somehow more calm now, more patient, understanding, I do not get upset so quickly now. I also noticed that, for example, I cope with stress better, sessions, exams, I learn better and I am not as afraid as I used to be. I simply don't think about that, but calmly prepare myself for them, go to the exam, and pass it without any problems. (practitioner for 3 years)

- And did you have a feeling that when you entered into yoga more, got more interested, that your closest surroundings also saw some change in you?

- Of course they have noticed. I hope that for the better (laughter followed by a moment of silence). I haven't drank alcohol for some years now, and this was such a big shock for my friends at the beginning, but after a year they somehow accepted this and now they do not even suggest alcohol. When I show up somewhere. "We bought a juice for you," they say. (practitioner for 14 years, yoga instructor, owner of hatha-yoga school)

The beginner usually compares him/herself to other practitioners: do I come up to them with my fitness? Generally, in hatha-yoga there is a rule not to compare oneself to others and to perform asanas according one's own possibilities. However, people who treat yoga as sport or gymnastics feel the need to compare themselves to others:

...And, of course, there are young girls coming, that are, believe me, so stretched that when I look, for example, how she bends some - God, my jaw drops - I never, in my life, 'til the end of my life... will be able to, but she is built differently than a boy - for a girl, because of her hip build, is easier to bend down or do the splits, right. I laughed in the end. In a previous class, I trained next to a girl... everybody has their own mat, there are common mats to be taken and the girl, such a position, where the legs are up... yes, "candle" it is called... it is so-called and the girl is doing this candle and she does not do a candle and splits her legs, right. And I say to her: Will you do the split? - "No problem." She did the split in the air. Then, my jaw dropped (laughter). I keep myself doing the candle, then she will additionally do the split. Well, I have no chance, there is no comparison and I am aware of the physical limitations, right. Apart from that, I doubt that I could stretch my joints in such a way so I could do that - in my whole life I have never done the splits, so why now am I supposed to do them? I can stand on my head, but with the split I have a problem. (beginner in yoga practice, age 50) 


\subsection{The phase of fuller recognition of the psychophysical effects, and ascribing appropriate meanings to them}

After perceiving the physical effects in the first phase of the development process of the practice, there follows a fuller perception of both the physical as well as the mental effects. There is a fully conscious connection of the physical development with the effects of psychic peace and calmness. This skill of connecting the two is a symptom of the beginning of the second phase of development in the practice of hatha-yoga. Some elements of this physical and mental process can occur in the first phase, but its fuller development occurs much later. This process (linking the physical and psychic developments) never ends, and what is important are the harmonious linkages of the psyche and the body:

It is such an ideal connection of the body with the spirit. The longer you exercise, the better and... the deeper it gets into you, the better you understand it. Although this is a very slow process, I am not an authority in this matter, but according to me, the soul of yoga, for me, the soul of yoga is this marvelous harmony. The fact that you can do this, that you are able to get such a cooperation between these two parts of you; such different parts; such an integration. (practitioner for 4 years, age 23)

And all the time, I practice like this, develop, go somewhere, gain different skills. I change, right? Because yoga is something... it is a process that causes changes, we change in the body, but we also change emotionally, mentally. As you maybe know, it is impossible to disconnect the body from the mind. When we are unhappy or mentally down, then our body is down too, right? It is said that people psycho-somatize and due to the suffering they start being sick. There is also such a nice saying that in a healthy body there is a healthy spirit. Working on our body, we influence the state of our consciousness, the mind, we feel happy, more balanced. And this is how we reach deeper and deeper, we learn about ourselves, our weak sides, we strengthen ourselves. (practitioner for 14 years, yoga instructor, owner of hatha-yoga school)

Even practicing yoga as a sport will not stop the practice's influence on the psyche. "There is no great difference when the spiritual aspect is omitted in the beginning. Asanas are psycho-physical exercises, not gymnastics. Sooner or later a man will experience in them inner harmony, integration, and happiness. He will empirically discover the spiritual layers of his humanity, which will lead him to further changes" (Pawłowska 2012). Yoga practice turns out to be an "agency" that influences the psychic changes in the individual, even against his/her will:

I, as a passive observer, that is a person that had never had contact with yoga, I believed it to be a kind of sport and now I hear that it is something different. 
Well, I have a friend that recently started going to yoga and she treats it like that that you can do yoga like that. She values this emancipation that you can relax, focus on the most important things. It also helps her a lot, but she does it mechanically. And absurdly, it turns out that even though she does not try to achieving that, still yoga influences her psyche as well. Although she might not be aware of this, does not see it, she perceives her world differently. (practitioner for 5 months, age 24)

The first yoga class, as it has been already mentioned, is not always a pleasant experience. Yoga requires a great amount of physical effort and the use of some muscles that might have never been used. The beginning of exercising can be very difficult, even after a few months of doing yoga:

After class, at 19.30, in the changing room there were comments about the exercises in lifting up legs to the perpendicular position (exercises for abdominal muscles), that they were hard and Michał overdoes them. But, these were accepting comments, with smiles...

Really, Michał usually gives us a hard time, sometimes we are on the verge of our endurance. I barely manage, try to survive until the moment when he says "maximum... enough." Exercises in one hour for the initial group are hard, intensive. Although the last exercises were mainly stretching, they were also exhausting. (8 months of practice of the author of this work, participant observation and autoethnography, May 20, 2012)

It is Saturday; today, I went to yoga in Źródliska Park. These meetings were organized by the company Yoga Centre, from Gdańska Street. ${ }^{6}$ The aim is advertisement, for two months in the park at 12.00 in the fresh air (they are free classes). It was ashtanga yoga, very difficult exercises. In the beginning, the instructor (4 years of practicing hatha-yoga and 6 months of ashtanga, I asked about it after class) explained what ashtanga is and the differences between it and hatha-yoga. In ashtanga, we have our eyes open, we do not hold long in a posture, and we exercise intensively, usually lasting for five breaths in asana. There are four levels of ashtanga, the last one is very difficult and only for the advanced. The majority practice on the first level. Between the exercises there was a constant intermission (the sun salutation in a shortened form). The exercises lasted 1.5 hour (I barely survived, I do not know how they are going to encourage people to do yoga, killing them with exercises in the beginning). I could not do some of the exercises, for example, the upward bow pose. I have had problems with these exercises for a very long time. My hands stop working.

The pace of doing the exercises was so fast that, really, you cannot think about anything else, full concentration on the here and now. There was even no time to observe how others exercise, but I liked the exercises. I really felt relaxed. This monotony of the intermission worked wonderfully.

After the exercises my muscles hurt and I was sore.

${ }^{6}$ See: http://www.yoga.net.pl (accessed: 17.11.2013). 
At the end I asked a question: How long did the lady instructor exercise? She said that the advances should be measured with our own measure, "how much further do I bend in comparison to previous times" or if I do a given exercise better. She stated that you should not look at the instructor or compare yourself with others. (8 months of practice of the author of this work, participant observation and autoethnography, May 24, 2008)

Such training causes muscle pains and cramps and sometimes you cannot do given exercises, which causes some problems with self-esteem. However, with more hatha-yoga practice, if a proper meaning is given by the practitioners (e.g., that yoga is a challenge, a healthy physical exercise, spiritual practice, etc.), then they stay with practicing it and they increase the intensity of the practice:

And I have to say that I did not expect such a hard workout (laughter), I mean, Iwona, a friend from high school, shed some light on it, and like I say, I read a bit, so I knew that yoga requires effort, but really, really after these first classes I was not alive, truly, not just that I was unable to cope with the correct performance of some postures, I also was unable to manage long enough in one posture, oh yes, because all my muscles hurt me and I have to say that I was not so flabby, I mean, I thought that it my fitness was not so bad, that I am more or less fit, sporty and yet, to my surprise, my muscles hurt me very much, and it turned out that I am terribly stiff and my self-esteem became really low (laughter). And the next day, I remember, wow, I was so sore, and Anka also texted me very pessimistically (laughter). But, after all I liked it, I treated it a bit like some sort of a challenge, well, because I do not like giving up so easily; so next week we went again, we went because Anka decided that ultimately it was not so bad after all and that she could use some exercises... And, in the beginning we went once a week, later twice, and now most commonly three times unless something comes up, because, you know, studies also take up some time. (practitioner for 3 years, age 22)

The meaning of recognizing your own body, getting acquainted with it, is an effect that you achieve at some point in the practice of hatha-yoga. The experiencing of the body is a constant process in yoga practice, and it includes becoming acquainted with your own body, for example, thanks to lasting in a given pose. It is a form of a contact with oneself. Achieving this moment can sometimes take years:

This contact with your own body was something that cost me a few years, such searching and... such... how to say it? I really wanted to understand what it is all about, this slogan. Apart from that I understand the words, well hmmm. (practitioner for 8 years)

Recognizing the body also refers to its different mental qualities. The body has, for example, memory; this means that the body can have 
some consciousness of itself. The subject of the action here treats the body subjectively, somehow it has an agency:

And this is interesting, that after a half a year break I only need a week and it is better than it was... to move, you are more stretched and the body remembers. (practitioner for 10 years, age 51)

Experiencing the body is connected with experiencing life (and life experience). The body reflects our current life situation. Working on our body in yoga, we work on our life:

Because everyone is different, everybody has different conditioning, different life experiences and different lanes, it goes and it processes differently. And even if it is happening not right, if we have some regress, stay in one place, then this also has some purpose, some reason, and in this moment it shows us something about ourselves and we can work with it. (practitioner for 5 years)

Recognizing the body is also recognizing, through hatha-yoga practice, the role of breathing in our life. You discover here the connection between the breath, body, and mind. The breath links with the movements of our body and the right breath with the mind concentration. Realization of this idea in practice is visible, especially in classes with an instructor who usually pays attention to calm and correct breathing. It is important to perceive the effects of yoga practice (in this case breathing) on life outside the classes and asanas exercises:

And, uh, I did not mention a very important thing, I think the most important thing in yoga, that is breathing. It would seem that breathing is really simple, it seemed so to me until I went to yoga (laughter) uh, to be honest, correct breathing is the basis of every well-done posture... Only then can the air affect every part of our body, breathing is synchronized with every move. And... and this correct breathing is also connected with this focusing, concentration... Such learning of the correctness of breathing is not easy, but later when we learn it, even outside of class, to breathe properly, that is by diaphragm then we realize that we begin to function differently, that somehow we have more energy, we are less sleepy. (practitioner for 3 years)

Breathing has many functions. A very important one is cleansing the body. Breathing is a regulator of our actions, their speed, and quality. All of this can be experienced while performing asanas, which can then be transferred into our everyday life. If the breath directs the rhythm of the exercises, then through analogy it also directs the rhythm of our actions in life and the rhythm of life in general: 
Breathing has many functions, and one of them is cleansing the body, liquidating the tensions. A breath in cleanses the mind and the body, and with the breath out all of these contaminations are let out. Secondly, and also important, breathing helps us to deepen the exercises, particular postures, it also sets the rhythm of the exercises. It tells what pace you should exercise in, how far you can move doing asanas, I mean, of course endurance and the capabilities of the body. (practitioner for 7 years, age 22)

Sole and specific breathing exercises (pranayama) are often done before or after asanas. Pranayama practice is about correct breathing, controlling and regulating the appropriate rhythm of breathing, sometimes on its withholding (Iyengar 1983). The practice of pranayama nourishes the healthy features of consciousness (vrtti):

I have said that exhalation empties the brain and pacifies the ego, bringing it to quiescent humility. When you empty the brain, you also empty the toxins of memory. With an exhalation and retention, you let go of resentment, anger, envy, and rancor. Exhalation is a sacred act of surrender, of self-abandonment. At the same time, we abandon all those stored up impurities that cling to the self - our resentment, anger, regrets, desires, envies, frustrations, and feeling of superiority and inadequacy and also the negativity that causes the obstacles to adhere to consciousness. When ego falls away, they fall away with it (Iyengar 2005a: 97).

The aim of pranayama is to make the respiratory system function at its best. This automatically improves the circulatory system, without each the process of digestion and elimination would suffer. Toxins would accumulate, diseases spread through the body, and ill-health become habitual. ... The respiratory system is the gateway to purifying the body, mind, and intellect. The key to this is pranayama (Iyengar 1983: 17).

You breathe through the nose with the whole lungs. The diagnosis of the body is also done by breathing, and the psycho-physical effects from pranayama are also perceived by the practitioners:

Madam, breathing, that is, these pranayamas are feeding the organism so there is no life without it! When someone learns to breath properly, then, Madam, he is healthy, simply long years. You need to air the organism just like a room, because it is known that when you do not air then you know - everything rots. The same is true in our body, if we air this organism, then really these illnesses do not stick, we really live a greater quality of life, well, really. I breathed my whole life through my mouth because I had some problems with this tonsil until today, and then later after starting yoga, I don't know - after the $3^{\text {rd }}$ or $4^{\text {th }}$ month - I noticed I was breathing through my nose. This was really a revelation... Well, apart from this, well, deep relaxation after all of this exhaustion, rest, and great relaxation for the organs... (practitioner, age 49)

The meanings ascribed to yoga change while practicing it, for example, from treating it as physical exercises to exercises calming down the psyche or as methods of fighting against stress. It is also connected with 
the increase of the practitioners' physical endurance, which allows them to better concentrate on the psychic or spiritual side of the practice.

When I started doing yoga, I treated it mainly as a good physical exercise, but I have to say that it is more, even greater when a person can disconnect from the outer world, separate from everyday life, forget about the problems and listen to the breathing, that is, focusing only on it, and finally, stop thinking. Yoga gives me a lot of relaxation, quiets me, allows me to calm down, de-stress, although I say it was not always like that. In the beginning every position was tiring for me, so I could not allow myself to do something like this, I got tired very quickly, but as my training time increases, my endurance increases all the time. (practitioner for 3 years)

Mental calmness has its opposition, which is psychic aggravation. This happens through aggravation of the body. Here, you also perceive the connection of the body and the psyche. That is why in yoga, according to the practitioners, it is very important to adjust the sequence of the exercises so that calming exercises follow stimulating exercises. All of this confirms the belief that there is a meaningful connection between the body and the psyche. The state of bodily stimulation influences the state of psychic stimulation:

...specific exercises have an influence, for example, on calming or aggravation, poorly done... poorly done, for example, in sequence. I think that we always have adjusted because we have a good teacher, poorly adjusted sequences can aggravate the body for example. (practitioner for 8 years)

Intermediate practitioners ascribe to yoga mainly the meaning of changes in the body and later in the psyche, and improvement of their physical and psychic wellbeing:

A person discovers such new physical horizons and knows their body better, because you feel parts of the body that you have not even known existed, and more importantly, you change psychically. You calm down, relax even. I, for example, learned patience while practicing yoga. I used to be an awfully hot-tempered person! Somebody or something made me upset and I exploded! And now (laughter)... really, I sometimes do not recognize myself (laughter)... this yoga is a very good thing. I am just a bit cautious when it comes to its influence on my life... but [my boyfriend Paweł], oh my Lord! Simply an oasis of peace! He is already a maniac about yoga. For him, it could be said it is his whole life (laughter)... you know every day 2-3 hours he does yoga, arguing with him is an art and a miracle! (practitioner for 2 years)

Even if the influence of yoga on everyday life can be unnoticeable, according to practitioners it exists:

Because it penetrates through one's life, right. Because it is some part of life, that... that you practice something there, I don't know, once or twice a week, but really it 
interfaces with your whole life and this has an influence and it is connected even with elements that seem unconnected, right? Through just the exercise, they are there. (practitioner for 5 years)

The physical and psychic well-being starts building a new quality of life. After several years of practicing yoga, some practitioners ascribe to yoga "improvement of the quality of life." This is a meaning that is connected with all changes, psychic and physical, and connected with an improved quality of everyday life (controlling emotions, lower stress, more creativity, and even the feeling of happiness, etc.):

Well then, what yoga gives me is surely that from the moment I practice I am happier, you know what I mean? Well, simply somehow what you practice yoga it transfers to my everyday life, I mean, I am no longer so tense, I do not worry about everything, it is a bit so that if I manage to achieve something in yoga, something that seems to be out of my reach, then I feel that it does not have to be like this only in yoga because, well, it's like, I got some wings (laughter). Because I have more faith in myself and now I look at the world more optimistically, with hope for betterment, and I await new challenges and I do not try, like in the past, to be unseen, hoping that I would not be noticed and I will somehow survive the situation... I also stress less now, constantly there is this calmness assisting me. I am more relaxed and it brings me great benefits, not only because of the fact that it positively influences my organism and the state of my spirit, but also I somehow do better in class, I have more ideas, I am more creative, and I am not afraid to speak up. I also learned to better control my emotions, these negative ones, in the sense I seldom get angry, get upset with somebody, and even when I am upset with somebody, then I either manage to keep it to myself or I manage to calmly explain what hurts me - what is wrong and why. I no longer need to shout to communicate; my family is also happy that I found yoga (laughter) because they live better with me now. I also have to say that under many conditions my life has improved, and from the perspective of these three years that I have been practicing yoga I have to admit that it was the best decision I ever made to try it. And in all honesty I can recommend yoga to others as a remedy, both for the body and the soul (laughter). Well, that would be all; I think I have nothing else to say. (practitioner for 3 years, age 22)

Later it transfers to life, sometimes when difficult situations get to us, then we know that we will cope with them because while practicing yoga many times we came across difficult situations and the challenges that yoga gives us. Reversed positions are such challenges - that you need to somehow put the world upside-down, right; which sometimes is very difficult, right? It's emotionally difficult and fear appears, but after some time we manage it, yes, we stand on our heads, on our hands, forearms, we do very strange things, that when we look at it from the outside, they seem impossible. But, because of what we do in yoga class, these impossible things, later when we encounter problems in life, then we approach them with more ease, yes, because somewhere it is written that if it seems that at yoga I am not able to do something and I did it, then in life I will do the same, with such an attitude and approach different problems, and just as in yoga I'll manage. That is why, well, yes... that is why yoga is wonderful (laughter). (practitioner for 14 years, yoga instructor, owner of a hatha-yoga school) 
Such a complete approach to one's interpretation of changes in life thanks to yoga practice is somewhat a confirmation of having made the right decision about practicing, and a justification for undertaking the huge physical and mental efforts connected with practicing yoga. By ascribing to yoga the meaning of improvement of one's quality of life, it means somehow accepting yoga as a necessary practice in everyday life. It is also an indicator of the appearance of the second phase in yoga practice. One's life attitude changes and his/her behavior in different situations changes as well. All the changes that happen in the life of an individual and in his or her identity confirm the initiation character of yoga:

When I comprehended the technique, then I entered into some, like, different layer, and then slowly I started seeing in me some peace, but not only during exercises. And this is the best part. This peace also started showing up outside the exercise room, in everyday life. It is amazing. It permeates into everything I do. I don't get upset at work. During class sessions or before exams I also manage. I used to be a very a hot-tempered person. I blew up every minute and now... well, okay, it happens, but when I am on the verge and I am about to get upset over such unimportant things suddenly, out of nowhere, this peace appears. I cannot describe it to you. (practitioner for 4 years, age 23)

The quality of life is connected with the psychic changes linked to the openness that in yoga is connected with the openness of the body, and is then transferred onto openness to life itself. The belief in the benefits and the marvel of yoga is socially strengthened by the opinions of other practitioners:

Whereas, if we control our mimics, and we do not do wild faces because it hurts us, or we do not grit our teeth, this is something... something new. First, second, third new things for the body, and this influences our openness towards the new in life in general ... Simply put, something new has happened. And for me, as I was microscopically interested in psychology, I got myself therapy then and put it all together, everything into each other. And, I can now say, for sure, that my life has changed - a lot - since that time. I have changed. For me it been much for the better, and from what I hear from people that I have contact with and who knew me earlier, these changes are marvelous and great in general. (practitioner for 8 years)

The change-of-life attitude and the improved quality of life are also reflected in a change of diet and the style of eating. One's diet is a form of addressing the body; thanks to this understanding yoga practice changes the way we treating our bodies and we eat different, healthy food. The need to use stimulants is also reduced: 
In general... I ate whatever... such unhealthy food, or such food that somehow... uhm... I don't know, like chocolate that is... increases the level of serotonin and improves the mood, and so on, cheese, and so on, such things that work to stimulate the organism... And this is the moment when it works more subtly, your sensitivity to your body, to your surroundings, and I noticed that you also start feeling what you eat... and you listen better to the needs of your organism, for example, you know better what is good and what is bad for you, uhm... so I, for example, acquired a taste for simple food, some simple products, with no chemicals, and I have a greater pleasure from eating such things. I do not want to eat fast food and drink fizzy drinks, and that's in general...

- And have you noticed that since you started exercising in such a way, regularly and more intensely, that you have a decrease in the urge for alcohol? Or maybe not?

- I think that too. In general, I am in such harmony with myself, I do not need these strong experiences, you know, you have this feeling of a more sublime reality, this feeling of yourself to such a point that it gives you so much, as if only excitement and experience, such subtle things that you do not need to snap somewhere... I mean that you no longer have to fool yourself with such strong things. (practitioner for 5 years)

A long-term hatha-yoga instructor talks about a proper diet (see the quote below) using specific terms such as: "toxins," "adding toxins," "organism poisoning," "awaking the consciousness," "auto-destruction through nutrition," "organism cleansing," "enlarging the space". These are terms of a language often used by the instructors and are delivered to the adepts of yoga so they could understand the reactions of their organisms and their inhibitions connected with the diet. The terminology connected with the diet is provided by famous gurus of hatha-yoga. For example, Sri Pattabhi Jois quotes the Upanishads: "When the food we take in is pure, our minds become pure. When our minds become pure, memory becomes steady" (2010: 24). This terminology and this knowledge shows us the close connection between nutrition and the state of the mind. However, other recommendations, for example, connected with limiting intercourses, are not advised and this subject is not mentioned by the yoga teachers I am basing my research on. It should be noted that such recommendations do, however, exist: "Too much sex leads the body, sense organs, and mind to become weak. If the mind and sense organs are weak, we can achieve nothing; our minds grow unsteady and are unable to do anything at all. Therefore, too much sex is to be avoided" (Pattabhi 2010: 25).

The observation of the body and its feelings connected with nutrition gives some basis for reflection. This reflection even takes on a "religious" character. The practitioners start wondering what is good and what is evil. This reflection starts from the thoughts about what is good for my body: 


\section{CHAPTER 4}

We also gain awareness of how important what we eat is. Yes, our body is not only a factory that does not care what we put in, some pot... it is about having speed for moving, for life. The quality of what we put into the body is very vital; we cannot treat our body like a rubbish bin, we put anything, and when we are still young the body manages well and we do not feel it, right? But, with time the amount of toxins gathered in the body accumulates and we unfortunately start getting sick, and anyway some illnesses appear. And not only at the level of bones and muscles, that we become stiffer due to some life experiences that tense us up, yes... it is known that stress goes over to the body, we tense up, yes, if it is long... if the stress holds on for a long time, we tense a lot and this tension stays permanently in our body. But bad nutrition also leads to the accumulation of different toxins in different muscles, cells, joints, and because of this joints become stiffer, everything becomes so swollen and set, and through the practice of asanas we can cleanse this, yes. But it is good when we clean not to add more toxins. Because we can cleanse practicing asanas, but all the time we will still, for example, smoke cigarettes and poison ourselves. And it will becomes so that here we clean ourselves a bit and there we poison ourselves a bit. And this will all be somewhat in one place. But after some time we will realize that you cannot work like this, because this is such a destructive action, yes. We poison ourselves for different reasons. Everybody has their reasons why s/he uses different stimulants and works auto-destructively. But we are aware of that, our consciousness awakes and some things that earlier seemed not important, become vital, and we take care of it, we wonder whether... what would be better to eat to feel better. Or, we observe, yes, when we eat such a pizza, that this pizza stays longer in us, that this does not digest, yes we have such an observation. If we eat pizza in the morning... I eat pizza sometimes... but I do not eat it in the afternoon because I know that then my organism will not deal with it till the evening and I will sleep bad (laughter). If I want pizza, I cannot deny it to myself, then I try to eat it before noon so I would have time to sleep. And so it is with different things, by observation, observation of the body, we observe the processes happening in the body and how the food we deliver influences it... and then I try to avoid this so I will not hurt myself. (practitioner for 14 years, hatha-yoga instructor, owner of a hatha-yoga school)

Thanks to exercises, more attention is also paid to the issue of nutrition before the exercises. Eating heavy meals before practicing asanas and eating directly before exercises is not recommended: "One of the participants of a feast left later in the evening during the exercises and did not return to the class, he felt weak because of having eaten fried camembert cheese in wild chanterelle sauce, as he stated" (participant observation, November 10, 2008).

The teacher teaches us to feel and name the feelings of our body. His words and forms of language allow us to feel some difficult exercises, and as a result different states of the body, in a positive fashion:

When pulling the body back from the wall on a line with the arms supporting against the floor, Michał approached me and when I simply could not do that exercise, he supported my back with his knee saying: "Straighten your legs, straight knees, hold 
with your legs, hands delicately support, you will feel stretching, you see how wonderful that is. Wonderful, right? Wonderful, right? Wonderful, right?" I answered, barely making a sound: "Wonderful..." The exercise seemed to be awfully difficult. After a while I managed to do it. The main problem was with the technique (straight legs) and breaking the fear barrier. I kept my position later on with a much smaller effort. (participant observation and autoethnography, November 11, 2008)

I was at a yoga class in the park. Michał ran the class. Today yoga was focused on searching for the balance. There were many exercises to train balance: tree, trikonasana, etc.; Michał, while doing the asanas, paid attention to the feeling of the body, especially the ones connected with the balance:

- "Feel how the back parts of your legs stretch, how your calves stretch (while doing the downward facing dog)."

- "Feel the balance between the right and left foot, feel how you achieve the balance, how you balance between the right and left side of your body. Feel how the center of gravity moves between the right and the left side of the body (the mountain position)." Michał also said a lot about achieving a balance between tension and relaxation. He talked about this while doing trikonasana: "Feel how your thighs work. Let the left leg work, but let abdominal muscles stay relaxed. The work of the body involves tension, but also relaxation. Relaxation is also work." When we were breathing, lying with our feet together, resting, Michał focused on breathing: "Hear and feel the wind. Now pay attention to your breathing. It is your inner wind. It cleanses your lungs." (participant observation and autoethnography, August 16, 2009)

The improvement in the quality of everyday life also increases the feelings of self-value, self-esteem, self-confidence, and perseverance, which changes one's attitude toward life and resolves many problems in everyday life. This all is ascribed to yoga. The individual has the awareness of acquiring new psychic abilities thanks to yoga, which builds his or her identity as a yoga practitioner:

For example, I don't know... let's say I walked with a proudly raised head ... then different things started solving themselves. It turned out that people looked at me differently, that they don't attack me, that I don't have to attack, that... I obviously name it using such sophisticated words, but in everyday life it has such a practical and sudden translation... normal, such normality. (practitioner for 8 years)

From what I've noticed, I am more self-assured in the positive sense. I know how to sell myself, I know my value and I have no feelings of inferiority. Simply put, when I have to do something, I give all of myself and I believe in myself, believe me or not, and this brings effects and surely this is the result of yoga because from the moment of starting the exercises some things really started changing in my life. When, for example, my friends during a session panic and worry about everything, at this time I simply learn, because there is no way I'll fail an exam if I study hard. (practitioner for 3 years)

- Yes, so what did yoga teach you?

- ...right...

- You talked about this fight with yourself. 


\section{CHAPTER 4}

- The word to describe all of this in one word is simply endurance... simply, that... you see the results after a longer while, but when you see them, then you feel this satisfaction that they are there and this brings something into your life. Often we expect quick results, and if something does not bring quick results then - you know there is such a model of our modern life, that everything's fast, right? And that is why we often lose energy if we don't see quick effects from something... you know, just achievements simply, then we don't want to get into that. And it is yoga that teaches us such endurance. (practitioner for 5 years)

It is also very important to obtain social skills. They are connected with attentiveness and concentration on the here and now. Heightened sensitivity into another person is the basis of being nice towards others. The interviews also show a high self-awareness of the researched persons and their reflexivity concerning their identity and what happened to it after starting the practice of yoga:

I became, if I say so, more aware and sensitive towards what happens around me. In the past I would not notice that. People are very busy now, rushing all the time work, classes, work, classes - and over and over again. There is not even a second to just look outside the window, to see how the world looks. Apart from that I try to smile more often at people, just for no reason, just simply be a nice person. (practitioner for 3 years)

With the progress in the practice many people want to deepen this practice. One of the methods is a trip to a solitary training centre, or trips to the roots of yoga, to India and schools functioning there:

Well, you know, what we deal with is only a part of what yoga really is. That is why Paweł (advanced practitioner, interlocutor's boyfriend) saves money to go to India to such workshops. (practitioner for 2 years)

However, some people run away at some point from the practice after perceiving the changes in their own bodies and psyche:

Anyway, she talks about this one (one of the Yogasutra) ${ }^{7}$ that misfortunes that will come... misfortunes that will come you should counteract them. And yoga is such a counteraction, to illnesses or... because there are misfortunes, yes,

${ }^{7}$ Yogasutra is a work describing the rules of yoga practice both from the technical as well as physical side. It consists of 196 aphorisms divided into four chapters (pada). They treat: Samadhi (the state of uniting with the object of meditation, smadhipada); means of achieving yoga (sadhanapada); powers appearing in the practitioner (wibhutipada); and a state of absolute freedom, emancipation from the circle of incarnations (kaiwalyapada). The authorship is ascribed to an ancient Hindi wise man, Patanjali, probably living in $3^{\text {rd }} / 4^{\text {th }}$ century (Iyengar 2002a). However, the texts of Yogasutra were created earlier, and Patanjali only worked them out again, and it is the last and important work of knowledge on the subject of yoga practice. 
touching us - when we are sick, we are not happy we want to get better, we cannot normally function and enjoy life. That is why it changes a lot, whether we want it or not; unless these changes are too difficult, yes? There are people who noticing the changes that happen have to stop practicing, yes, they have to stop practicing because these changes are difficult to cope with, it turns out that there is too much to change and they are not ready yet. Right... for example, if someone is really addicted to cigarettes and knows that it is not good for him, he is aware of that, sometimes people run away from this. They run away from the practice because they do not want to face what they have already noticed. They have noticed that they hurt themselves, but they are not ready to change it. Of course, if they went on practicing, then it would help them to achieve the goal, become stronger. (practitioner for 14 years, yoga instructor, owner of a hatha-yoga school)

\subsection{Phase of fuller recognition of the spiritual (para-religious) aspects of hatha-yoga}

Some sort of problem may arise for practitioners connected with their attitude towards the spiritual aspects of yoga. As is known, originally yoga was linked with certain spiritual and moral values that need to be obeyed. They are set out in the so-called Eightfold Path of Yoga by Patanjali (Iyengar 2002a). First, there should be morality, and only then exercises as asanas. If, following B. K. S. Iyengar, we imagine yoga as a tree, then the roots would be moral values (yamas). Breathing exercises and meditation are also elements of the practice.

Thus, the elements of yoga at the very beginning are bans, socalled yamas: not using violence (ahimsa), not lying (satya), not stealing (asteya), lack of desire and controls over sensual desires (brahmacarya), and lack of greed (aparigraha). These are followed by recommendations (niyamas). These are something like the trunk of the tree of yoga: cleanliness (sauca), contentment (santosa), self-discipline and eagerness (tapas), self-knowing (swadhyaya), and giving oneself to God (Iswara-pranidhana). Giving oneself to God obviously points to the most to the religious aspect of yoga practice. It is about focusing on the godly aspect, although many Western teachers do not accept it as a religion so as not to discourage the willing, who maybe at the same time confessors of different religions, from practicing yoga.

The bans support the development of cleansing the body and mind; and recommendations are the signs that should be followed in order to strengthen the practice.

Yoga practice is described in the remaining elements of the Eightfold Path: the practice of yoga postures (asanas, branches of the tree), breathing exercises (pranayama, the leaves of the yoga tree), with- 
drawal (pratyahara, bark of the tree), concentration (dharana, the sap of the tree), meditation (dhyana, the flower of the yoga tree), clean awareness (Samadhi, the fruit of the tree of yoga), which is the climax when we forget about ego and the soul penetrates every element of the body. Samadhi is clean awareness, connection with the object/objects of meditation and achieving a state of unity with the universe (Iyengar 2002b; see also Eliade 1997: 106-110; Iyengar 2002a; 2005a: 3-37).

The yogi simply observes the fact. "It is raining," he may think or say, without desire or judgment... The yogic purpose of pratyahara is to make the mind set up so we can concentrate (Iyengar 2005a: 100-101; see also Iyengar 2005b).

The spiritual elements point to the goal of yoga, which is seeing the soul. Performing asanas allows one to find and experience the unity of the body, mind, and soul. This is so-called active meditation (Iyengar 2002b: 149). At the end of the development of practice, posture is carried out in a perfect way (ibid.: 149). Yoga allows one to withhold the movements of the mind (citta-vrtti-nirodha). Then it is possible to observe the space between the emptiness and fullness.

\begin{abstract}
Through restraint of the movements of consciousness, a space is created between thoughtlessness, between emptiness and fullness. Observe that. By observing the space between the two, you realize that chittavrtti is different from chitta - the movement of consciousness is different from consciousness itself. Patanjali says that when consciousness become quiet, when the contemplative state of attention has been attained, through asana, pranayama, dharana, and dhyana, then the consciousness realizes that it has no light of his own, because it cannot act and witness at the same time. It is dependent on the reflection of the light of the soul (Iyengar 2002b: 134-135; see also Pattabhi 2010: 8).
\end{abstract}

Consciousness thus depends on the soul. There is some dualism that yoga tries to show the subject by practice, but the final aim is the connection of the soul and consciousness.

This very short description of the formal process of yoga practice and its goals ${ }^{8}$ may create some sort of a problem for the more aware practitioners who undertake the attempt to explain their relation with the practice and the philosophy itself. Yoga teachers rarely openly and verbally address this plot of practice during yoga classes, nor after.

The practice of asanas does something to the body of practitioners, and this marks the beginning of the appearance of some phenomena. These phenomena can be explained by the language of medicine and physiology of the body; however, the moral-religious side of yoga can

${ }^{8}$ Eliade writes widely about religious rules of yoga $(1997 ; 2004 ; 2009)$. 
only offer as much as to give some food for thought. This thinking is often supported through the literature about yoga, together with the terminology used therein:

\begin{abstract}
Nerve centers stimulate breathing, you know that when you breathe differently, as I read here, that some nerve is closed, some space opened then this simply influences the well-being, the mind. These are not any magical things, this is that when you go to do fitness, then you leave with serotonin or some other euphoria - I do not know which euphoria. Nobody is shocked, the same thing is with yoga, this is also movement, the same as in any other movement; I personally believe that this is, according to yogis - according to people that make their religion out of this - well, this is something created by God. Even if not by God, then surely by wise men, even if, well, let's say, I don't have to believe that this is created by Siva, but that it was worked on through thousands of years, or hundreds let's say, that one, a second, and a third came up with this idea, I mean one did, the second continued, the third, and so on and so forth, through hundreds of years, even thousands because it is still being developed. Now, maybe these changes are not so important as they used to be in the beginning; but, I think that this is done not only as in fitness, directed towards losing weight and experiencing some euphoria and, in general, body building - but from the beginning it was based on some grounds of some philosophy... If it was done according to the commandments of yoga, then first, for example, one needs to clean the consciousness and act in a moral way. Do some things and don't do other things. And it is written what should be done, what should not be done, more or less the same as in Christianity. Just like in any other religion. And you also need to be good, I mean not only morally good, according to human commandments; there is nothing new there. Only then you approach the practice. So only this, even if I start exercising, but I come across some brochure that tells me that, then it makes me think, for example. (practitioner for 8 years)
\end{abstract}

Some practitioners demonstrate a selective approach to the whole philosophy of yoga and yoga practice. Yoga, in this situation, delivers a wide array of rules and practices that are the basis of free choice of an individual, choices connected with some elements from the set of rules. This is somewhat a private choice; it is done independently from institutional conditions and compulsions, the latter of which is often present in traditional and institutionalized religions. ${ }^{9}$ Modern religious topics, apart from self-expression, the ethos of mobility, sexuality, and familiarity, are also a subject of individual autonomy (Luckmann 2006: 147-154). Free choice, i.e. agreeing with the interests of an individual who describes for him/herself what yoga is, is an expression of individual autonomy. It can be some philosophy and/or style of life with religious elements, but also yoga can be treated as just physical exercises. Even if we point to the freedom of choice of some elements of yoga practice and discard its religious character, then, in the end, as

${ }^{9}$ Compare: Knoblauch (2006): 23, 25-26. 
in the example below, we can also point to spiritual elements where the awareness of oneself also gives the "awareness of the world, connection to nature and harmony." Yoga becomes a privatized and individually understood para-religion, or quasi-religion. The individual finds grounds for his or her individual choices in the network of direct contacts, for example, among friends, colleagues, an environment of common interests, family, etc. These individuals find themselves in a private sphere; primary public institutions no longer assure legitimization for these autonomic choices $^{10}$ :

Yoga can be done in disconnection from the philosophy it is connected with... It can be treated only as some sort of physical activity. It does not need to be treated as a whole, anybody can choose, take from yoga something that is the most interesting, can focus on its different elements, such as breathing exercises, meditation, Buddhist or Hindi philosophy.

- What, according to you, is the most important in yoga? In its influence on a person?

- I think that such key elements, as you could call them, are concentration, balance, flexibility, and here I mean both the physical as well as mental... Apart from this, a very important element is auto-control... this is what yoga gives, such awareness of your own self, your own body through this focusing on a given exercise, given posture. If I do not concentrate, I will not do a given posture properly... Apart from such awareness of oneself, I think that yoga also gives an awareness of the surrounding world, of one's connection with nature, harmony. (practitioner for 5 years)

Yoga seems to be helpful in privatizing and individualizing the values of spiritual areas. People have different needs, and different practices are good for different people; the choice of those most appropriate for an individual means the "privatizing of yoga practice" (see also: Chapter 1, where the problem of privatization of religion is described):

It is about living according to your values, postulates, your ideals, so that this life would be ethically moral, so every day you could look pleased with a smile on the face on your reflection in the mirror, and it should be an honest, real smile. I think that it gives me a lot... helps me. (exercising for 5 months)

Because everybody needs different things, right; maybe this is good for me because it agrees with me; because I have such a personality and practice yoga in such a way; or with my attitude towards life; it is good for me, but maybe somebody else needs something else, and it is not known how this road will go; that maybe one day

${ }^{10}$ Compare: Luckmann (2006): 145-146. Although "religion requires a religious community," we also have secular agencies that help in alteration and/or confirming the plausibility structure of social reality, such as psychotherapy or institutions of political indoctrination (see: Berger, Luckmann 1991: 178). There are also other institutions, such as yoga, meditation, Eastern dancing, and karate schools. 
it will go in such a spiritual direction, or maybe remain at the body level, and such benefits are for here and now. And you do not know, it cannot be disapproved of. (practitioner for 5 years)

Spiritual elements are pointed at when, for example, talking about breathing. Breathing points to meditational character of yoga's physical exercises. Focusing on breathing results in the state of "not thinking."

After some time of practicing yoga, some auto-definitions connected with yoga begin to appear. In general, it is difficult for practtioners to define who they are in yoga practice. For sure they are not yogis, because this requires enormous devotion and practice so strong as to exclude everyday life - first and foremost requires full time to devote to the practice. Generally, most practitioners define themselves as "exercising/practicing yoga." Yoga is, for them, something more than physical exercises, but also something less than a spiritual practice:

- So what is more important, the body or spiritual development?

- Well, rather both I guess, because you cannot disconnect these two. Only that in the case of yoga, together with spiritual development there is also physical development. This is the simplest way it can be described because not always when you exercise do you also develop mentally and here, well, I am not saying that I went to yoga just to somehow get into the philosophy or to meditate or fly in general. And I am not a yogi after all, I only practice yoga. (practitioner for 20 years, with breaks)

The auto-definitions that appear in the third phase of the development of the practitioner are connected with the acceptance of oneself as a person. Yoga exercises are a form of taking care of yourself. Being good to yourself is, after some time, also connected with being good to others. Here, the element pointing to hatha-yoga as a para-religion becomes important because you work on the change of your personality. A greater awareness of such types of spirituality is shown by yoga teachers and persons who have been practicing for a long time:

Well, OK, I work on my body, I make time for myself to practice, to look after myself. And everyone, I think, when they look after themselves more, loves themselves, then they start feeling better. Everything that surrounds you in life better, I don't know if I said it right, but when we love ourselves, then people who love, maybe they come closer to each other. And they love us so much because we love ourselves. And we radiate. We send radiation. There is something like this, but such specific quietness, such a greater happiness; such a larger acceptance of oneself. (practitioner for 12 years, yoga teacher) 
What is also important in yoga is the opening not only of the body, but also of one's worldview, which again points to the para-religious character of the practice. It is then about the creation of some psychic attitude that enables spiritual reflection:

\begin{abstract}
Opening to this whole worldview, meditation... maybe the ability to accept the things that we normally don't accept because... it is so, yoga leads to emancipation, to cleansing, this is, I guess, the most important part. So it should not be approached only as body exercises, but to open more to the philosophy, so the mind would also benefit. (practitioner for 20 years, with breaks)
\end{abstract}

It is difficult to disconnect the spirituality of yoga from the bodylines and feeling of the body. This connection, frankly speaking, is the most important aspect of yoga. The body awareness has a spiritual character:

- And does yoga give you; anything spiritual?

- Well, I mean, that if these were more regular exercises then probably it would give more because you enter a deeper contact with all of the muscles. You find out about the existence of muscles that before you would have had no idea about, because they are not usually activated; only during yoga. And you need to find out, get used to, and learn to breathe. And, honestly, a deeper breathing makes you enter a different... the mind works differently, you start thinking, focusing on what is happening, and when you stretch every muscle, how every muscle hurts (laughter), and you try to close this pain, move away, so it is, well, on the verge of . . . maybe not spiritual, but on the verge of spirituality. (no data about the length of practice, age 23, woman)

The contact with the body involves not only recognition of the body itself. The sole recognition of physical body is a springboard to understanding reality in the shape of specified mental states, for example, being here and now or disconnecting from thinking and not referring to oneself, or being in the past and the future tense (memories and projections). The body is thus a very important tool of work on the psyche, which turns out to be just a layer of spiritual practice, because the connections with spiritual religiousness are pointed out only marginally and partially. The relationship of the corporal practice with the spiritual and psyche is pointed out by Iyengar: "Do not think, therefore, that asana pertains only to the physical sheath. There is a total involvement between the three sheaths of the body (annamaya kosa), energy (pranamaya kosa), and mind (manomaya kosa)" (2005a: 103).

And here they tell me to overturn. Contact with the body is about that which I am to look for... these movements in the body and the way to do so, to do it and to feel comfortable because then it is ideal, that the position is comfortable... and effort- 
less. And also that I can, more importantly, meditate in this posture and only in this. Of course, there is a slogan "meditate in the posture." Well, this is quite a concrete suggestion. Of course, if you achieve it, I congratulate you. But I only think about what I do in the here and now. I think that for majority of people this is such a challenge, not to think about millions of things such as why he does not want me or why... why somebody likes me or does not like me, what I eat and if I earn enough, and so on and so forth. Or about parents... why they were like this or were not like that... different things, everybody has... or how to wangle something and so on... different things. It is very difficult to think for an hour and a half only about the here and now... such a typical slogan of Eastern religions. And, this, this is an answer to, I think, some question that was or will be that, well, this is something new, a characteristic of yoga being present in the here and now. This is the thing that is apart from the clear physicality, such typical, corporal effects. (practitioner for 8 years)

But, well, yoga teaches us to focus in a given moment on one thing. And this is what it teaches. And I think this is the ideal, that the person knows how to (silence)... well yoga teaches such specifics... I mean, not specific, such as I think I have a cool attitude to life, but so that in a given second you are aware of that second and not to reach to far away because all of this can change, because in every moment somehow what is going to happen later has an influence, so when I am aware of this moment that is now and I live it so positively, then I know that what comes later will also be positive. And I think that, well at least for me, such a perfectness in yoga to be able to know how to live and feel in a given moment. That is even... that is what these exercises teach - that I focus on a given posture and completely disconnect from the thoughts and plans that are in my head, that are running through my head. There must be a focus on what I'm doing in a given moment; on what I think, on where I am, and such direction of these thoughts, feelings, everything that I do in a given moment. (yoga and fitness instructor)

Being in the here and now and gaining the awareness of the present is an extremely important element of teaching the religion of the East. Such teaching is important in, for example, Buddhism (see: Zotz 2007; Scherer 2009), but also in hatha-yoga. Meditation which allows you to focus on the here and now is a part of hatha-yoga practice. Moreover: "Meditation is integration - to make the disintegrated parts of man become one again" (Iyengar 2002b: 144). What's more, Iyengar states that the different names given to meditation relate to the same state of meditation. There is just one meditation:

In many religious practices, one will find meditation and different ways of working with the emotions and desires. You may hear of Zen meditation and think that it is something different from meditation in yoga. But meditation cannot be called Hindu meditation, Zen meditation, or transcendental meditation. Meditation is simply meditation. Remember that the Buddha was born in India and was also a student of yoga (Iyengar 2002b: 15; see also: Zotz 2007; Scherer 2009).

Thanks to this it is possible to build one's own syncretic spiritual attitude. 
Some find certain values that influence their mental state in the spiritual aspects of yoga. Humility, as a value, becomes a mental feeling that is achieved by yoga, it is connected with attaining some attitude which is the openness attitude. It has a mental character, but in connection with "humility" it acquires a spiritual virtue. These values can be discovered quite early during asana practice; however, they mature together with the advancement in practice:

However, this opened me. I also remember this feeling, so new and really interesting, so fresh, creative; in general, the notion of humility... these were the first classes. One of the first... first months that I started feeling how cool it is to be humble that I need to fight, I pay so much I demand, for example. That somebody cannot care or that it can be different than I imagined, this is a completely and differently understood humility... completely different. Such daily humility, a humility that I started discovering as if it just came to and I then named it. I'm telling you, it is humility. And, it was a fantastic feeling of such a great life-relief that I hung around it for several months, because it was wonderful. I mean, of course this costs... it is not so that this is so wonderful that I walk as if I was in love. No, this costs, there are also painful feelings, this is also a fight with oneself, it is... like moving any stiff thing. (practitioner for 8 years)

The mental state achieved by practicing yoga allows one to perceive reality differently. What used to be a problem for an individual is no longer such a problem. Inner harmony gives one the strength to cope with everyday life and its problems. Although it is difficult to unequivocally state the influence of yoga upon the life attitudes (on the mind) of practitioners, mentioning the change of life style, together with the change of managing routines, indicates however a full change and full influence of hatha-yoga on the life of a practicing individual. Such changes have a spiritual character and are connected with the identity transformation (para-religious activities), not only mental or physical. It is interesting that the spheres of mind and life are mentioned here at all, even though the practice is connected mainly with the body and physical exercises:

With time, it is possible to notice its influence on many other spheres of our lives. I, for example, started paying attention to completely different things... some problems that in the past seemed so big that for sure I would not manage them, suddenly became unimportant. Well, in general, yoga gave me what I wanted, that is this inner peace, such an inner harmony, but also strength. Strength, because I thought to myself that nothing is impossible. If every time during the class I overcome my boundaries, the boundaries of my body, then in my life I can also cross these boundaries. So I think that yoga can be such a life style, way of thinking and acting. What is also important, I think, is getting a good teacher to start with, one who can make you interested with all of this, give some things because, unfortunately, not every- 
body can do that. And that would be all. It is hard to talk about something that is connected to the mind and it's hard to name some states - they need to be lived, I guess (laughter). (practitioner for 3 years)

Thanks to yoga practice we gain... we attract the inner light that solves our problems. We are often completely unaware of how to do that and we are shocked that it happens so fast. And now, it's apropos to talk of what we have to give up when doing yoga. With time, when I follow the path, I discover that some factors that cause my... that cause me to develop slower. And in a natural way, if someone wants to have improvements in yoga, he or she tries to eliminate those factors that limit him. That is why many people practicing yoga give up stimulants such as tobacco, alcohol, not to mention drugs, as well as certain styles of life that do not agree with us. We start changing it so that our improvement, our perfecting, and the satisfaction that follows are deeper. Some people give up, for example, life... how to say it... they stop going to discos or doing some forms of entertainment that are not good for them.

- And, in your case, what were the factors that you found as limiting you?

- From the very beginning when I started practicing yoga I stopped drinking alcohol. Completely. And if earlier drinking alcohol gave me pleasure, then now it is something really unpleasant. Awareness grows in yoga. I am more aware, my mind becomes clearer, is able to look at everything from a better perspective. However, alcoholic intoxication has a completely opposite effect.

- And you feel that, this change?

- Yes, absolutely. However, why do people drink alcohol? Because if someone does not do yoga, he often has problems to control his emotions and the quickest form of such emotional relaxation is reaching for alcohol. That is why many people become addicted to it, because it gives them a momentary kind of freedom from their emotional problems. However, people who do yoga have no need to drink alcohol. Of course, everybody is different, I cannot say that everyone that starts doing yoga stops drinking alcohol. However, from my experience, most people that I know have similar experiences. Lately, I also gave up drinking black and green tea. I felt it was not good for me. (practitioner for 14 years)

The spiritual aspect is also underlined in commonsense theorizing about the essence of yoga, where Eastern philosophy of thinking is mentioned; for example the notion of illusion (maya) and liquid reality. Here you also leave outside commonsense thinking into the sphere of mystical experiences (that this "is more a sort of feeling"):

And uhm... I think that this is also because of history, being very varied in comparison, true to Asia so even the teachings that Jesus passed on somewhere there can be also... also can be probably people who later on passed on, interpreted them differently and this interpretation started somewhere moving away from what actually... what actually this record was, started being systematized, uhm, because of this limited, closing in some images, right? And such a big Eastern weapon for such thinking is that there is this battle with these images that this notion of such an illusion, this maya that surrounds us and that... that people have this awareness, I mean these people, I am saying uhm, let us assume that people have this aware- 
ness of the illusiveness of everything - that these are only concepts, these are only images and that the notion and the image always exist in a constant way while the reality is liquid, that it always exists in this process and that words and images serve only as a way of transmission or as a, uhm ... something that directs you, right? As such a... this hand that points to something and that you do not look at this moment at the hand that is pointing but at what it is pointing at. And this truly such a way of looking, a level of such abstract thinking that goes beyond words, right? It is more such a feeling and I think that it can be so attractive in the Eastern sense that the same things can be reached using the same path, right? Well, I do not know. (practitioner for 5 years)

Yoga teachers talk more about the spiritual aspects of yoga. Physical aspects, for example, breathing and movement, breath and body co-ordination, have spiritual values for them. There is energy in breathing (prana) that, according to the interlocutor, can be identified with God. A thesis can be made that the more advanced the practitioner, the more attention is paid to spirituality:

\begin{abstract}
And when we breathe, we breathe in fresh prana, fresh energy let's call it. And this energy is needed by us, we need this energy to cleanse everything. And this energy, it can be said in Hinduism, or yoga, is thinking that God is in this "prana." God is... I don't know how to say it in Polish so I will say it in English. In an atom, you have the electron, and you have the proton that goes around. In the electron you have the negative and positives and everything that holds that little atom together - God is in there. God is also everywhere. He is even bigger than the universe. So you've got God everywhere and God is considered to be this energy, this energy that is "prana." So breathing is very important because the whole time you need more energy. You need more energy to keep at what you're doing, to keep the positions. So at some level, I started from the level of spirit where you're breathing in God, you're breathing in the energy. You're breathing in the life force. And you need life force to keep living. You don't need food so much, that is, you need food, but you really need breathing. You can't live on without breathing - maybe for five minutes. This breath is really, really important in this sense, but also in the sense that we do not do such an exercise just to move, for the muscles to move, but this is, to be honest, such a focusing that we move but we add breathing to this. (practitioner for 12 years, yoga teacher)
\end{abstract}

Teachers sometimes also talk about a conscious search for the state of enlightenment and Samadhi (state of pure awareness and/or blissfulness, complete connection with the object of meditation). This state is their aim and the aim of yoga practice. The awareness of these types of aims definitely more vividly points out the quasi-religious nature of yoga practice:

But... well, if I could already tomorrow, then already tomorrow I would ask for Samadhi, and Samadhi is so-called nirvana. This is a complete connection with God. Then really no yoga is needed, nothing is needed, you can only sit and the 
man is... he feels as if because all the time we are connected with God, this God is, whether this is some kind of a man with a beard, which some think so, or if it is energy that is in this air. I believe that we are constantly connected to it. Only that we, as humans... we need to think that we are separate, that we are something different. But, in yoga, what we strive for is this self-realization, so we... so this third eye would open. And this third eye is, we see it, we see it all the time, that we are a piece of God, we are joined to this, there is no separation between anything. And we are as if... as if this energy flows through us. And this state is called Samadhi. This is bliss. I don't know... something like that. This is what I am looking for, striving for (laughter). From tomorrow, please (laughter). (practitioner for 12 years, yoga teacher)

The same teacher denies that yoga is a religion, however, the statement below (by the same teacher) points to the quasi-religious character of yoga and, at the same time, the "private character" of every religion (everyone has their own religion). So first the religious character of yoga is denied, and later its quasi-religious nature is pointed out:

I mean, it strengthens, maybe something like that, that they come to yoga as Catholics and a bit, they are a bit scared because yoga is such a strange religion, and yet it is not a religion, here nobody says anything, this is all the time a work on yourself. And this is such a test on yourself. And what somebody wants to do with religion is his or her own business. Sometimes, people move away from their religion because they start yoga, but later on it is as if they have found their true religion I think, so that everyone, let's say, can be Catholics, but everyone has their own religion. (practitioner for 12 years, yoga teacher)

This quasi-religious or para-religious character is especially obvious for people who searched in yoga from the very beginning for spiritual and "religious" threads. Yoga was for them a certain point of achievement after searching for this right spiritual path and satisfaction in life. Yoga is for them searching for contact with the soul, the divine element. Interesting here is the fact that this contact is, for many years, treated as a form of perfecting oneself, which states the "invisibility of religion" and its subjectivity, some feeling of the transcendental nature of their corporality, achieved during the practice. The individual often chooses his or her own the way to achieve this contact with his or her "own soul." Spirituality is here a question of autonomy of the individual, his or her private choices shaping the "patchwork" definition of religion. Every individual has his or her private, individual path to reach contact with his or her true "I":

In the beginning, I looked for this satisfaction in philosophy, in religion, and later in many religions. And even later, I came across books about yoga and courses that were the answer to what I was looking for. Because yoga is... yoga itself is about finding, one can say, the perfect part of each of us. In everyone there is something 


\section{CHAPTER 4}

really deep, pure, perfect. And in many cultures, different religions, it is called differently. And that is why some people call it soul, some call it infinity in the heart, some call it God... and there are people who experience contact with this, I will call it from now on let's say "soul"... contact with the soul they experience in a very religious way. Other people experience this contact and don't describe it as a contact with God, but as a form of perfecting, for example... the aim of yoga is always the same, that is contact with the soul, contact with our true inner "I" - something deep and perfect - but there are many methods to achieve it. You can choose to follow one method, however, if we move from one to another, well, sometimes they might seem to be contradictory, going in different directions. Admittedly they go towards the same goal, but in different ways. (practitioner for 14 years)

A thesis can be made that the more advanced and experienced a practitioner is, the more often he or she uses other elements of yoga than just the practice of performing asanas. They sing, for example, mantras and meditate, set their own definition and make a routine of practice:

My method is the heart yoga. It is yoga based on meditation every day in peace, and on singing mantras. This is the basis of the path of yoga I follow. And... every day in the morning I meditate, at least a few... several minutes, and this gives me great strength, this gives me a lot of positive qualities which stay with me the whole year. These are such qualities like peace, happiness, enthusiasm, calmness, harmony that come to me in the meditations. (practitioner for 14 years)

We come across an opposite sequence in a different statement. Below, the presented interlocutor accents first the spiritual character of yoga, then she distances herself from the religious aspects of this practice. She points to the spiritual elements when describing breathing. Breath is, for her, an indicator of the meditational character of the physical exercises of yoga. Focusing on breathing creates the state of "not thinking." Breath for the advanced practitioners has a spiritual character:

- And what then about breathing? You said that it is the most important.

- Well, yes, because with... with meditation then the breathing helps one could turn off the thoughts. The moment one starts focusing on breathing and as if following its course then he focuses on this breathing in such a way that he stops thinking, that this breath becomes his thought and if do not pay attention to this breath and... frankly, there is nothing (laughter). And this is it, that there is nothing (laughter). (fitness and yoga instructor)

Next, the interviewee distances herself from the religious aspects of yoga practice (ritual aspects such as singing hymns) and from the instructors who introduce the spirituality of yoga, and gives a simply pragmatic reason for practicing it (helps reduce sicknesses, possibility 
of mental quieting, ability to direct energy to specific places, harmonious development of the body). Yet this distancing from the religious aspects of yoga also means recognizing the spiritual aspects of yoga. It seems that there is some need in people who are already advanced in the practice of asanas - but not fully identifying with the spiritual or religious aspects of yoga - to describe/explain one's own position on the matter:

While I, for example, practice yoga, I do not consider myself to be a typical yogi that sings hymns and practices yoga to learn meditation or, I don't know, change my awareness, no, that isn't so, this is so... well, I can't even say why I practice. There are many reasons. That it is for me it's some form of relaxation, that I believe that yoga can help in many ailments, in many illnesses, just thanks to such emancipation and ability to direct energy to a given place. So I say that although it is difficult... call it such a clean yoga. It cannot even be called this. Because even being in a course and having to do with such people, somehow this does not fully convince me. Because a yogi sings hymns in honor of Patanjali and I am not going to sing hymns in honor of Patanjali, although I am grateful to Patanjali that he created it. (fitness and yoga instructor)

I do not engage in these spiritual experiences and such things, and it pushed me away from one center. I am a practicing and believing Catholic, so other philosophies are not needed by me for anything. I have no intention of changing either my denomination or the faith that I was brought up in. But, really, I like accepting yoga and the teachers such that I accept that... well, there are such dominating tendencies in yoga schools in Poland that focus on the harmonious development of the body. (practitioner, age 53)

It turned out that the lady running the class is cranky and gabbled something about yoga, about meditation, about some joss sticks, that you need to quiet yourself down and be in a completely different world, I tell you. So the beginnings were a bit funny and difficult. Later, it was not so bad anymore. (practitioner for 3 years)

Treating yoga as a religion is inseparably connected with corporality, with the connection of what is sensual with the body. If this happens, then there is also a change in the life of the individual, mental changes are noticeable, there is an energy flow and greater mind concentration, thus yoga could be defined by one interlocutor as "religion" (in our sense, a para-religion) since these changes are too big to happen in a short period of time without the integration of a transcendental factor:

I would like you to tell me why you started practicing yoga, how long has it lasted, what did your life look like before, and how does it look now, being a yogi.

I practice yoga for... only for 5 months. Yoga introduced many changes to my life. It started shaping my mind, calming it. It greatly enabled me to concentrate, gave me more energy, life energy, to get up, enjoy life. It allowed me to do something like linking my body with my mind, because yoga is not only a technique but also some 
sort of a religion, that is, it postulates ideas that accompany yoga. This gives a lot, it really gives a lot. The person concentrates on what is the most important in life; you pay attention to what is the most important in life. Earlier I was a really scatterbrained person, and on top of that I had such a fear in me. I was nervous, hot-tempered, and not calm, not concentrated at all. And in this aspect yoga changed my life completely. I can concentrate on anything I want in any given moment. (practitioner for 5 months)

In previous classes I went to this guy, he was rather funny for me, maybe because he was such a devoted Buddhist and, lying down on the ground when you have your eyes closed, and he tells you: "Now your eyes are like stones, something, your body becomes soft, your stomach becomes something... you do not feel it at all..." so it was so sect-like for me because... because I simply did not believe in it, but now this woman that runs my classes does not talk like that at all, just simply tells us to relax and this meditation comes out of you. (practicing student of ethnology, length of practice not noted)

Another element of the attitude of distancing oneself from the spiritual aspects is the comparison of yoga with one's own culture, and keeping connections with that culture. Yoga is used here selectively, as physical exercises to gain mental peace:

Generally, yoga is some philosophy, but I, well... (moment of silence) this is not the moment yet, for me, or maybe yoga gives me now so much goodness, gives me peace, balance. For now, I guess... I try to find some comparison with our culture because I was brought up here, in a completely different culture, with completely different standards, Catholicism and such, my parents, I mean my father is very religious. Well, it is difficult to switch just like that, well, maybe it's just difficult to give up something just like that and jump into something new. I know that some are fascinated by this and do so, but I still try to connect it somehow, find some, I don't know, equivalents? Dang it... (laughter) well cope with this somehow so I won't have to jump into such deep water... philosophy. (practitioner for 7 years)

However, later on, the same practitioner mentions that it is difficult to run away from these spiritual elements, as you come across them all the time during practice:

One is, for sure, on a certain stage, you cannot run away from the philosophy. Well, at yoga class itself it is difficult to run away from it because it is known that the teachers mention such things when you go to camps, I have gone there for many years, there is also such an introduction to the meditation, some things... and, well, yoga philosophy needs to be mentioned as well. (practitioner for 7 years)

Discarding religious aspects often has a paradoxical character - as they profess to get rid of the religious sphere, in their justifications the yoga practitioners refer to religious threads. These threads have a selective character that again points to the individualistic constructions of 
this "para-religion" for private use. Discovering one's life aim using yoga has a spiritual aspect. Shaping the subject in childhood by yoga surely had an effect on the current life attitude of the interlocutor below:

In the beginning, you asked if it was some life philosophy. It seems to me that the interpretation is free, different for everybody. I, for sure, do not link it with a clearly religious sphere, although maybe spiritual, I mean, yoga for me is a journey inside me. The journey which is learning oneself, discovering your life goal, solving problems. Some, for sure, treat yoga as some life style in a wider meaning, that is physical sphere-exercises, spiritual sphere-meditation, and there is also a third element which is the everyday sphere - an appropriate vegetarian diet. I am also vegetarian, but yoga had no direct influence on that. In my case, yoga really influences the physical sphere and when it comes to my character, interpersonal relations, in general, the whole way I look at the world, not eating meat, well then I can't exclude that the reason for this was adolescence. Now I understand it so, that yoga maybe was some factor, after all I was about 15 years old when I started being interested in it, and for sure it had a great influence upon the person I am today. In the end, I spent time in such a society, I observed a lot of others, how they behaved, how they approached it, I was a young girl, I still am (laughter). This surely shaped me somehow, pushed me this way and not the other. (practitioner for 7 years, age 22)

Sometimes questions about the spiritual aspects of yoga invoke irritation among those interlocutors who distance themselves from the religious aspect of yoga. Although up until now we do not have quantitative research, I believe however, at least on the basis of my research, that the majority of those researched do not openly attach any meaning to the spiritual experiences. In the U.S.A. this situation seems to be different, as a meaningful percentage of practitioners attach some meaning to the spiritual experiences in yoga practice. The surveys in the U.S.A. show that the majority of Americans do yoga to feel better and de-stress, $37 \%$ look for spiritual experience, and 5\% decided to practice following a doctor's suggestion (Augustyn 2010). This shows that doing yoga in our Western culture can be a touchy issue, because religious identification is very important, as is building an unambiguous religious identity (e.g., Catholic). Religion seems to be connected with health and a frame of mind. Religiously-oriented Americans lead a more hygienic and pro-health life style. ${ }^{11}$ Research shows that very religious Americans feel worry, stress, sadness, and anger to a lesser degree than moderately religious and non-religious respondents. ${ }^{12} \mathrm{Be}$ low are the remarks of the interviewer after the end of the interview:

${ }^{11}$ See: http://www.gallup.com/poll/145379/Religious-Americans-Lead-Healthier-Lives. aspx (accessed: 5.01.2011).

12 See: http://www.gallup.com/poll/144980/Religious-Americans-Report-LessDepression-Worry.aspx (accessed: 5.01.2011). 
The women gave very complex answers. She had a lot to say about yoga, but only in the physical aspect. After the interview she underlined that the questions about spirituality made her a bit upset because along her path she encountered people who desperately tried to convince her of this spirituality and philosophy connected with yoga, which she does not want. She treats yoga as a health aide, not a spiritual quest. (interview with practitioner, age 53)

\section{The socio-religious context of practicing yoga}

The socio-religious context in which yoga is practiced is very important. Poland is a Catholic country, with the great majority of people professing Catholicism (95\%), about half of which regularly attend religious services (at least once a week) (Boguszewski 2010). Hence emphasizing the spiritual aspects of yoga by practitioners surely goes against the widely accepted and acknowledged spiritual and religious values in our country. However, it needs to be pointed out that Poles also believe that it is possible to cast a spell on somebody (34\%), that distant telepathy is possible (39\%), that some people have clairvoyant skills (59\%).

An overwhelming majority of those researched (87\%, including $49 \%$ of whom are people who are sure of it) believe that every religion is just as good if it helps the human to go through life the best possible way. Almost three fourths of those researched (70\%) claim that is God worshipped in many religions, no matter what he is called, in reality it is the same God, a slightly smaller group (67\%) are those who claim that all religions, in fact, have one goal towards which they lead human beings (Boguszewski 2010: 5).

In the context of this acceptance of other religions and beliefs, hatha-yoga can find its space in the religious system created subjectively by the individual, even if it can create some anxiety among the representatives of institutional religion, i.e. priests.

Priest Jacek Prusak also refers to the problem of the spiritual aspects of yoga in an interview with a journalist from Gazeta Wyborcza. In the Catholic society, introducing other religious threads is dangerous for its integrity, although the work with the body can better prepare people for prayer. The above doubts of some practitioners in this context, from their point of view, thus seem justified:

From time to time, clients ask us if yoga is against the Catholic religion. Bubicz even recalls a woman that heard from a priest that the worst are so-called reversed positions (candle, headstand) because then Satan can enter through the anus.

- It is rubbish. Should we, because of Satan, sleep standing - protests father Jacek Prusak from Cracow. Hatha-yoga itself, which is about body exercises and opening the energy channels, is not against Christianity. This is a spiritual gymnastics for 
everybody. Work with the body can prepare one for a deeper prayer. The church has nothing against it. However, when it comes to higher levels of yoga, greater caution and carefulness is suggested.

Father Prusak warns the believers against the cult of their own bodies, against narcissism. And what's more, against advanced breathing techniques that are supposed to lead to a visualization referring to non-Christian symbolism: "It is about techniques that are to allow entering into the state of consciousness where ego melts into the Absolute. For Christians, this is not described Absolute, but the presence of the Secret that Christians call God" (Staszewski 2009).

It needs to be underlined that the breathing techniques (pranayama) are an integral part of hatha-yoga (see: Stinissen 2000; Matus 2003). Pranayama is "a regulation of energy and life force through rhythmic control of breath" (Iyengar 2002b: 178). Pranayama is also a spiritual practice, it releases divine energy (ibid.: 127, see also 133). The idea of prana comes from the sacred Hindi books, thus it must be a religious concept:

According to the Upanisads, prana is a principle of life and consciousness. It is equated with the real Self (Atma). Prana is the breath of life of all beings in the universe. They are born through and live by it, and when they die, their individual breath dissolves into the cosmic breath. Prana is the hub of the Wheel of Life... It is being (sat) and not being (asat). It is the source of all knowledge. It is the Cosmic Personality (purusa) of samkhya philosophy. Therefore, the Yogi takes refuge in prana (Iyengar 1983: 12).

During the practice certain feelings can give rise to interesting questions. This is what some researchers have experienced:

I am after pranayama. I breathe more consciously. Viloma on a breathe in requires the division of the breath into four parts and stopping four times the breath in. These stops should be longer, according to what I heard from Michał during the instructions (breath in - stop, breath in - stop, breath in - stop, breath in - stop). Today I elongated them. It was more difficult than yesterday. During savasana I observe the breath. I noticed that at one point I was observing the observing subject. Who is this, observing the observer? Are these still my thoughts? It is about observing the breath, but suddenly the observer of the observer appears... Who is it? What to do with him?

Later, we sang the ohm mantra, each after each other, so the sound would constantly assist us and caused vibrations. I really felt vibrations in the body; everywhere I heard the singing of particular participants. I listened to Michał's singing. After that, there was meditation. I observed the lights of the candles. I sailed away. It was nice. (10 months of practice of the author of this work, participant observation and autoethnography during a week retreat training in the mountains, July 10, 2008) 
"The dangers of yoga for the Catholic faith" is where religious aspects enter into yoga. This is why the church gives warnings. Yoga, excluding the religious aspects, is not dangerous and it can even be used instrumentally for practicing the Catholic faith, and meditation should be linked with God and the Holy Spirit. Meditation is also a part of yoga practice (Iyengar 2002b: 55, 118, 144-148). The practice of mantra singing is also linked with individual and contextual choices. The individual shapes his or her own individual practice, choosing from the mosaic of the spiritual religion of the East what is the most comfortable and useful in a given moment:

There are also really concrete mantras that we can use. For example, when we are really upset we use such a mantra that calms us. When we experience depression, we use a mantra that will cheer us up, give us good spirits. So, there are many mantras. If somebody feels the vocation to such practice, he can have a really cool adventure. (practitioner for 14 years)

Singing mantras is a form of meditation. However, it resembles some Christian rituals and can be treated as a competitive order:

The Church, however, gives a warning: you should meditate with the connection of the Holy Spirit.

- If it is a warning, then there is a danger. What kind?

- Meditation is like a knife; it can be used for good or bad purposes. Different forms of meditation can open a person to the works of evil powers. Sinking into this atmosphere, occultism, contact with evil powers can lead to possession. Also, practicing meditation by atheists can be dangerous. Especially when they meditate in order to achieve the feeling of emptiness, not being, like Buddhists, because emancipation can open one to the works of evil spirits. Some people, also priests, claim that mantras refer to Hindi Gods.

- And it is right. That's why when it comes to spiritual dimensions one should follow the theologians. For Christians, the solution is to practice yoga which third step is a Christian meditation. Today everyone can teach meditation and yoga, people follow the name and there are tens of thousands of teaching methods. Some of them have religious elements, and this is dangerous. My advice is: practice yoga, but when it comes to meditation, go back to your God.

Of course, yoga, if misunderstood, can be a window for occultism, but let us not pour the child out together with the bath (Ziółkowski 2010).

Maybe the cultural-religious context results in fact from that in the auto-presenting strategies of yoga schools on the Internet websites (among those analyzed by me), out of 172 yoga schools only 24 (14\%) describe some religious aspects connected with the school. Among 172 yoga schools, 63 (36.6\%) describe some philosophic aspects of 
yoga practice. Definitely the most important element in the auto-presentation strategies are health benefits $(83.7 \%$ of schools describe the health benefits), which seems to be more acceptable in the Polish cultural and religious contexts.

For Father Pereira, deepening the hatha-yoga practice would be an ill-understood yoga. Beyond that, many people moved away from yoga when they started experiencing spiritual aspects, some feelings, visions, intuitions later confirmed by facts. These experiences frightened them and resulted in moving away from yoga and returning to their primary and original religious experiences, for example connected with Catholicism. ${ }^{13}$

Like meditation, singing mantras can have a religious character. Here is the text of Gayatra' mantra sometimes sung after asana exercises during retreat sessions of yoga:

AUM BHOOR BHUWAH SWAHA,

TAT SAVITUR VARENYAM,

BHARGO DEVASAYA DHEEMAHI,

DHIYO YO NAHA PRACHODAYAT.

The English translation of the mantra is: "May the Almighty God illuminate our intellect to lead us along the righteous path."14

Positive acceptance of these new psychic experiences or spiritual results in the end will deepen the practice. Such forms are often at the centre of trips to ashrams of India.

For some hatha-yoga practitioners, trips to India are not only connected with the practice of hatha-yoga in Puna. Some of them visit and even stay longer in Ashrams, where meditation is practiced. Some of the practitioners that the researcher met during his fieldwork went to the "Vishvatmak Yangli Maharay Ashram" in Kokamthan, where they meditated for a longer period of time (two weeks to three months). These are meditations according to Om Gurudev, meditations for Atma (Divine Soul, Ego, Cosmic Energy, Universal Consciousness, God; I quote here from a leaflet given during meditation in one of the yoga schools, data from participant observation): "Meditation is the easiest way to come closer to Atma and experiencing it. This experience will give us eternal happiness called Sat-Chit-Ananda (truth-consciousness-happiness). Atma has no form. But, it should be visualized in an appropriate form. By meditation we can become One with

${ }^{13}$ See film: Yoga can be dangerous, http://www.youtube.com/watch?v=1hKM_1Uuim4 $\&$ feature $=$ relmfu (accessed: 8.05.2012).

${ }^{14}$ See: http://www.eaglespace.com/spirit/gayatri.php (accessed: 11.11.2008). 
Atma." ${ }^{15}$ Such a road to spirituality of hatha-yoga practitioners be imagined: after the practice of asanas and pranayama starting the meditation, including meditation directed to experiencing Atma.

In general, meditation is a further and "natural" deepening and widening of asanas practice in hatha-yoga. Some practitioners treat it as relaxing and instrumental. ("Many scientists make discoveries and invent things thanks to meditation... thanks to meditation we can make right decisions" - statement from the above-quoted leaflet). But there are also those who gain contact with Atma. "If we meditate Atma in our hearts, we become aware and experience that Atma is universal."16 In one of the yoga schools there are weekly meditations, where people with different philosophical-religious orientations and motivations come together to meditate. In these meditations some religious syncretism is visible, as different traditions and religious symbols are connected:

Meditations were done in the form of some built altar (on a table) where there were candles standing, the symbol of a star, somebody placed an image (picture) of merciful Jesus with a radiating heart and a picture of a woman saint from India (I do not remember her name). Behind the altar there was a big, one-meter by two-meter image - a visualization of Atma. It is a man with androgynous features, long arms reaching the knees, with long hair, and with marked chakras. The image of Jesus was also placed by some lady, who had earlier asked if she could meditate in Jesus intention. The teacher agreed to that. (data from participant observation, February 20, 2011)

The radiating heart of Jesus is convergent here in some sense with the rules of meditation and visualization, when we imagine the teacher or the image of Atma in our hearts. Thus, the image of Christ can be also useful here, especially for people for whom he is an important character.

\subsection{Conclusions}

Yoga enables contact with oneself. This contact in hatha-yoga is mediated by the body. The work on the body is done to change the way of functioning of the mind. This skill of working on the body is obtained through asana practice, which defines the identity of the practitioners. Verbal auto-definitions can differ here, together with the distance from the spiritual/religious aspects of yoga. Thus, becoming a yogi is a longterm process, ending in achieving the Samadhi state. It is a state attainable by only a scarce number of practitioners.

\footnotetext{
${ }^{15}$ See: http://www.vishwatmak.org (accessed: 20.07.2014).

16 Ibidem.
} 
The process of becoming a hatha-yoga practitioner 137

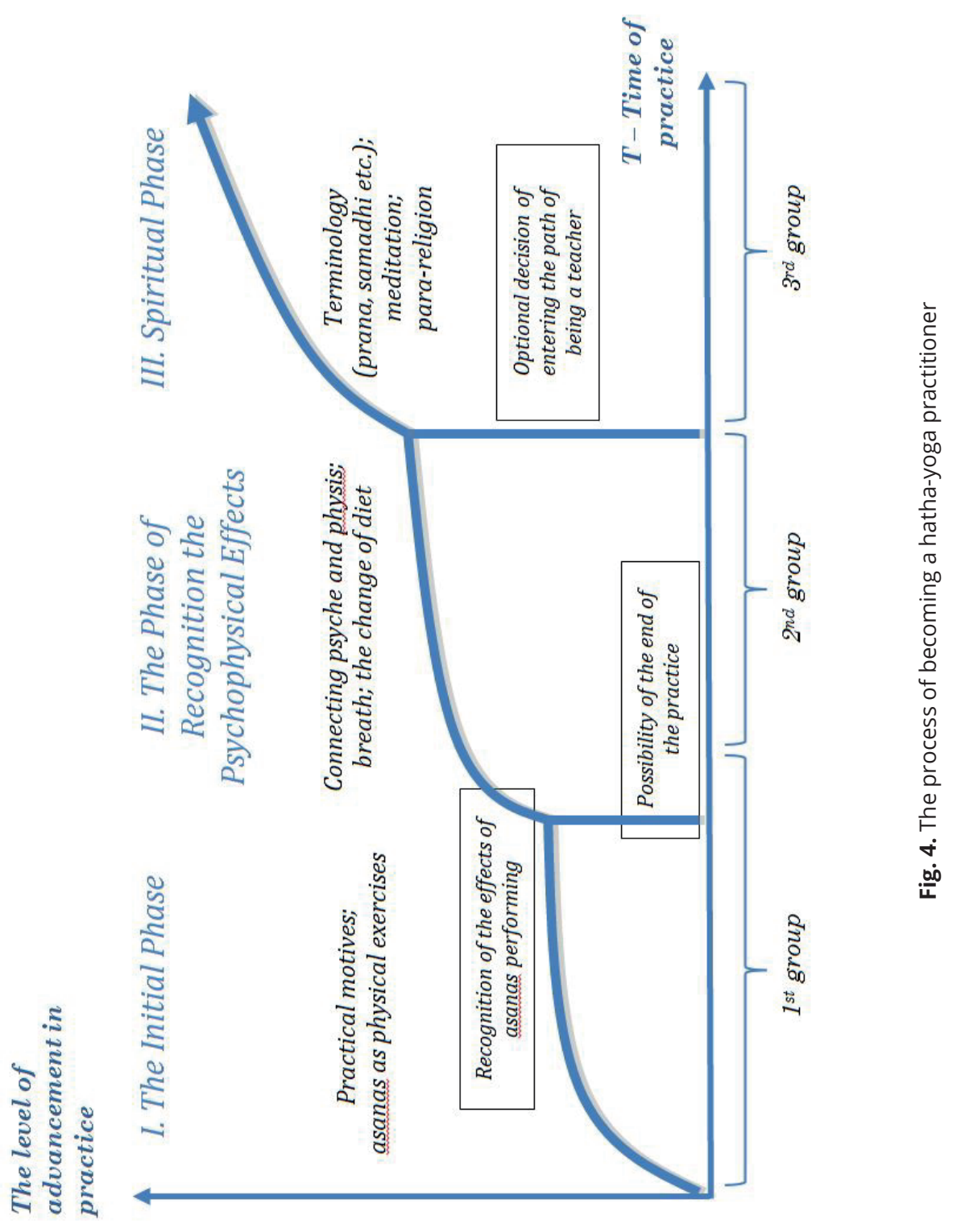


The level of advancement in yoga practice (the ability to perform asanas and the knowledge about yoga and ascribing spiritual aspects to it) describes to us how the individual defines his or her identity, as well as the hatha-yoga practice itself. The level of advancement allows for distinguishing three groups of practitioners, which are distinguished by gaining certain levels of advancement. Some practitioners stop at the first stage (introductory stage) of becoming a practitioner. They are then yoga practitioners whot build their motivation to practice it based on practical motives connected with corporality, that is perception of the body, health, and calming the mind. Very vital here is the individual's perception of the effects of the practice (see fig. 4).

A very important part of reaching the stage of practitioner of hatha-yoga is to obtain a certificate. The Ramamani Iyengar Memorial Yoga Institute (RIMYI) offers courses with 13 levels of certification, and each level has its own syllabus and exams where the practitioners should write a theoretical paper on asanas, pranayama and the anatomy and physiology of yoga, demonstrate asanas and pranayama, and demonstrate teaching skills. ${ }^{17}$ This system of certification is spread around the world and carried out in many countries in institutions approved by RIMYI. However, this certification refers only to those persons who want to become teachers. Practicing yoga is not only connected with teaching yoga, but with the commitment to the world of yoga and its rules, as we could observe in our research.

If the proper conditions appear, practitioners can move on to the next, second stage of the process of becoming a hatha-yoga practitioner. This is connected with having a fuller perception of psycho-physical effects of the practice, that is connecting the breath, feeling the body, and the mind. The ability to correctly perform asanas becomes greater. A new quality of everyday life is then perceived, connected with control of emotions, decreased stress, greater creativity, and even feeling happiness. The practitioner's self-esteem increases. Some practitioners stop at this point. However, after achieving above values, the individual starts to reflect on the spiritual dimension of yoga, and then she/he moves to the third stage of the development of the identity. Yoga then appears to the individual not only as an opening of the body, but also as a "philosophical openness." Work on the body becomes a spiritual practice. Some of them feel a greater sense of humility and openness towards the world. These people already know the hatha-yo-

${ }^{17}$ See: Ramamani Iyengar Memorial Yoga Institute (RIMYI) offer: http://www. bksiyengar.com/modules/institut/rimyi/certi.htm (accessed: 20.07.2014). 
ga terminology and its philosophy. This third group of practitioners are mostly instructors and yoga teachers; most advanced in the work on the body (practicing asanas).

However, becoming a "practitioner of hatha-yoga/asana" is a never-ending process, as the work on the body and learning how to do the asanas continues, and you can never say that you have reached the zenith or end of these skills. Similarly, this is connected with the perception of the effects of the practice (physical, health, and mental) and discovering the spiritual dimension of yoga. Here there are always new elements, together with the need to distance oneself from other religious and/or spiritual aspects of yoga. What allows us to recognize the identity of 'practitioner' (although always in process) is the ability to perform asanas and perceive the effects of practicing, as well as becoming aware of the spiritual aspects of yoga (either through their acceptance or rejection). These three elements somehow end the process of becoming a practitioner, although they just begin the process of becoming a yogi, which will probably never be undertaken in the case of the majority of the practitioners researched by me.

As a consequence of such an intensive practice, the body links with the mind and consciousness to finally become at the same time both the mind and the consciousness. We can quote here a certain statement by a yogi (Swami X) from a discussion with a Buddhist Zen master, Seung Sa. Swami interprets this phenomenon of a working mind as follows:

Seung Sa said: How do you keep your mind during yoga?

- We link with our mind and our inner "I." And the mind should not have any objects. When the mind enters this inner "I," it becomes one with the inner "I." But, when it leaves, it is separated. The mind is not a separate existence, it is not a reshaping of anything, it is nothing but awareness. When the common awareness focuses on its own and acquires the form of outer objects, then I call it mind. And when the same mind goes inside and becomes "I," then it is again awareness in itself. It focuses and spreads (Seung 2006: 205-206).

Putting aside the differences between these two spiritual schools, it can be said that the mind becomes, during yoga practice, awareness itself. It does not focus on outer objects. This way the feeling of self-value and at the same time humility is born and/or strengthened. According to those researched persons who accept some spiritual aspects of yoga, work on the body and contact with one's own body allow this. Focusing through the body on the inner "I," removing the 
outer world problems from the present awareness during practice, also shows some illusiveness of the outer world. Yoga gives distance to this, of course, if the physical exercises are adjusted to "spiritual" interpretations. They often have the character of para-religious interpretations, not strictly religious; there is no religion appearing even with the awareness of the relations of the body, mind, inner "I," and sometimes God-awareness. However, the very perception of these relations has a not fully and scientifically explained character for some of the practitioners. 


\section{CHAPTER 5 \\ Visual transmission of knowledge and the meaning of corporality and gestures in the social world of yoga practice}

\subsection{Introduction}

In the chapter ${ }^{1}$ I deal with research problems connected with the issues of the transfer of body feelings and phenomena connected with embodiment.

The problem of the body and the embodiment became very popular in sociology since the 1990s. However, the research on the embodiment and the body have a rather theoretical character, and even if empirical, then by the use of techniques based in direct and indirect communication. Observational techniques are seldom used (see: Waskul, Vanini 2006; Jakubowska 2009). This chapter shows the trial of linking the observational techniques with the interview techniques and is close to the naturalistic research on the practice of using the body (Schubert 2006; see also Laurier, Maze, Lundin 2006; Heath, Luft, 2007a; 2007b; Konecki 2008; Knoblauch 2008), although it widens it by individual experiences of the body researched with the usage of interview techniques which characterize the approach of symbolic interactionism (Schrock, Boyd 2006) or phenomenology (Brandt 2006) or the model of research through mutual experiences (Wyka 1993). The photographic representation of many activities are common in contemporary society where the visualization of many social processes, selves and everyday life activities (e.g. hobbies, sport activities) are presented in social media and in many public places (Bogunia-Borowska, Sztompka 2012; Sztompka 2005; Drozdowski 2008; Drozdowski, Krajewski 2008).

A very important element in popularization of hatha-yoga in the modern world was the technical possibility of visual reproduction of yoga and yoga training. The development of photography, relatively low price of photography, and the possibility to print it in yoga textbooks and popular magazines are an important technological element

1 This chapter is a broadened version of "Wywiad na podstawie filmów w badaniach praktyki jogi - na przykładzie badań cielesności i gestów w społecznym świecie praktyki jogi" [Movie-elicited interviews in yoga practice research - the example of research on embodiment and gestures in the social world of yoga practice] (Konecki 2010). 
contributing to the social development of the yoga world in the modern civilization. The transmission of yoga is currently based on "photographic realism" which causes lessening of the interest in aspects of "traditional" esoteric yoga.

Singleton (2010a: 164) quoting the views of John Putz from the work Photography and the Body shows what photography was for the modernistically oriented worldview. Photography here is somewhat a continuation of empirically oriented Renaissance that accented sensualistic research and understanding of the world. Photography was such a tool of an impersonal and non-emotional, "objective" way of transmitting knowledge. Photography has also changed the status of the body of an individual in society so that currently the individual is more self-aware of and can observe it on its own. The video recordings, movies also helped in the process. It may have been also a way of expressing self-awareness of the middle class.

Photographs were also used by ethnologists (e.g., British on the demand of the government) to learn and document different races and classify them. Some believed that the superiority of the European white race was shown. Thus, the photography was a tool of control and power over colonized "others." It also applied to showing India in the photographs of travellers and journalists. In the end of $19^{\text {th }}$ century and in the beginning of $20^{\text {th }}$ century, traditional, travelling yogis were not shown in the photographs in a positive light.

Apart from specifying such functions of photography one more can be added, the one that interests me the most here is the transmission of knowledge about hatha-yoga and the possibility for visual training. Already in the 1920s of the previous century there were precise photographs of asanas done by Hindi yogis. Photography and its development allowed, according to Singleton (2010a: 167), for the discontinuing of the tradition of hatha-yoga rather than for breaking up with it. The photography causes an epistemological breaking up with the tradition because there happened to be concentration on the body (asanas) and diminution of the interest in the esoteric knowledge. "Subtle" hatha-yoga that was presented in the traditional Hindi art, for example, with anatomical paintings of yogis showing places of the positioning of chakras and connections and channels of energy flow (nadis), disappears in realistic photographs showing the practice of asanas (ibid.: 170-171). Yogic body moves from conceptual, heuristic tantric existence to the perceived, objective, and empiric and realistic body in contemporary hatha-yoga. Such presentation of the body is also currently present in photographs of hatha-yoga positions in the textbooks and training films picturing that practice of yoga. Such types 
of presentations can be seen in the films from the year 1938 where Iyengar shows the vinyasa of yoga. ${ }^{2}$

The photographic presentation of yoga seems to be an important strategy in the auto-presentation of yoga schools. On 172 researched websites of yoga schools in Poland in 38 cities, $81.4 \%$ of schools have photos on their website.

Among the 172 researched yoga schools, 65.7\% have photos of asanas on their website. Bringing closer what yoga is by using photographs is a very important tactic in the promotional strategy of schools. Among the 172 researched yoga schools, $41.3 \%$ of schools have photos of important people in yoga on their websites. Asanas seem to be more important than the authorities in visual strategies of auto-presentation. Following data also confirmed that, $55.2 \%$ have photos from class in the school on their websites. Showing the practice seems to have a bigger power of persuasion. The visualization of yoga practice concerns then also the layer of predicted reception of the pictures of yoga practice as more interesting for the recipients than the clearly textual presentation. The presentation is sometimes enforced with the photos from open air classes. Among the 172 researched schools, 45.3\% have photos from open air classes on their website. However, the photos of the school itself (buildings, rooms) seem less important for the owners of yoga schools (33.1\% have photos of the school itself on their website).

Among the researched websites of the 172 yoga schools, 33 (19.2\%) have photos from other countries on their website, these are photos of yoga practices or places and surroundings of this practice. 38 yoga schools (22.1\%), however, show photos from India.

An interesting cultural fact is also the way of presenting yoga in magazines propagating it. In the magazine Yoga Journal on the front page there are usually young, slim, attractive women. Next to the photos there are mainly headings connected with the improvement of health, the silhouette, and mood thanks to practicing yoga. Photos enhance the persuasive power of verbal presentation. Mainly women are shown, often doing complicated and difficult positions solo $(66 \%$ of photographs). Photos are also, for the most part, showing white people (78\%). Visual presentation is thus directed mainly towards the women of the white middle class. ${ }^{3}$ In general, in these photographs are not shown yogis from the country where you look for the roots of yoga. The

${ }^{2}$ See: http://www.youtube.com/watch?v=lmOUZQi_6Tw; http://www.youtube.com/ watch?v=I0oq04oyO3Y (accessed: 11.03.2012).

3 See: http://thesocietypages.org/socimages/2011/09/14/exclusion-and-americanyoga (accessed: 16.10.2011). 
ancient images or even of more modern Hindi creators of yoga are not in the front pages which main aim is to sell the product that yoga is rather then propagate its original and spiritual aspects.

The material presented in this chapter comes from movie-elicited interviews and also on analyses of training films. These films were, just like the above photos in Yoga Journal, created for commercial and training purposes. The movie-elicited interviews (based on the video or documentary or feature film) can activate in the interlocutor some sphere of the memory and imagination that are vital in remembering the events taken for granted or that were believed to be verbally nontransferable. One can leave outside here what was visually and superficially observed as deeper meanings activated by pictures.

In my research concerning feeling the body in hatha-yoga, I used training films. These films showed in a realistic form, "objective," somewhat reflecting the physical reality of movements, how to perform certain exercises (asanas), and sometimes what the consequences for the organism of doing a given exercise are (for commercial yoga see: Budilovsky, Adamson, Flynn 2007 and for traditional yoga see: Iyengar 2002a; Eliade 2004; 2009).

In the movie-elicited interviews based on the training films concerning hatha-yoga practice, the interlocutors practicing hatha-yoga describe their feelings connected both with their practice, as well as with the reception of the watched films. I wanted to see how knowledge transfer about yoga practice in the aspect of feeling the body is constructed by the practitioners. It is difficult to catch the feelings of the body while practicing as the practitioners are so involved in the exercises that at the same moment it is difficult to get from them any verbal message regarding these feelings. That is why I decided to use the picture (training films) to inspire this message and see how the knowledge about the experience of the body based on the pictures is constructed. The pictures themselves convey some knowledge about the body and its work. However, the practitioners answering the questions connected with the film pay attention to the feelings connected with the body and not only talk about general impressions and consequences coming from practicing yoga as it is in the unstructured and narrative interview. Conveyed knowledge is knowledge based on a subjective, individual feeling, but its verbalization typifies it and allows intersubjective understanding.

The aim of the chapter is to show the results of the research on experiencing the body in yoga practice and conveying the knowledge on the subject of this experience. In the chapter, I mainly deal with the process of embodied experiences and the description 
of categories connected with it. I am interested in the reconstruction of experiencing the body during exercising, that is, practicing asanas, different body postures where different parts of the body are in every position different and specifically adjusted according to one another. Yoga practice that I am describing here is hatha-yoga practice according to B. K. S. Iyengar where the practice of asanas and pranayama (techniques of breathing) are the main practices, popularized in many yoga schools in the world. For the majority of teachers, yoga practice is some program of exercises encompassing the whole body and mind. Concentration on the body and its strength, flexibility, balance, endurance, and general healthiness is a commonly used argument of schools encouraging practicing yoga of this type. In popularizing and advertising hatha-yoga, the benefits for the body are shown, for example, that it strengthens muscles and reduces weight, makes the body more flexible, improves the balance, "gives never-ending energy," soothes different types of pains and stiffness (Budilovsky, Adamson, Flynn 2007: 5). Even though hatha-yoga has also a spiritual dimension, and even religious (Eliade 2004; 2009), the focus on this dimension in our Western cultural context is definitely smaller than the basic guidelines of yoga foreseen.

Bernt Schnettler (2008: 129-131) distinguishes three types of visual knowledge:

a) the knowledge of the experts about what visual is (esthetics or iconology); this knowledge is possessed by specialists in different domains, such as art historians, philosophers of paintings, but also graphics, typesetters, poster creators, etc.;

b) visual knowledge regarding non-verbal, bodily forms of expression passed on only in a visual way; this knowledge is remnant of embodied knowledge because just like the latter one it contrasts with verbalizations: "Thus, it describes these elements of human ways of expressing that can be verbalized only with a lot of difficulties losing at the same time a lot in meaning" (Schnettler 2008: 129). Very crucial here is the understanding of the meanings of body expression and gestures during interaction or during motoric show, for example, dance or martial art training;

c) about visual knowledge it can be also said that what is socially believed to be knowledge, is distributed through the use of new audio-visual medias, such as distance learning, and/or by using Power Point presentations.

The knowledge passed in the films which I partially talk about here is a visual knowledge belonging to the second type of meditation according to the classification by B. Schnettler, that is, to non-verbal, 
bodily forms of expression. However, this knowledge cannot be fully passed on the visual path. As it is stated by B. Schnettler, not all visual shows have the character of the signs, context of the transfer and the way it is received are very important, who and where and how it passes on and receives this knowledge. It seems thus that there is also one more type of visual knowledge referring to the body gestures that links different methods of knowledge transfer. Often, the knowledge about the feelings of the body, the embodied knowledge, so it could be conveyed, needs a lot of hybrid sets of means of transfer: picture, words, gestures. Such an example is the transfer about the experiences of the body during doing asanas. The picture shows technical aspects connected with the body movements and their coordination, words help this technical description, and sometimes there is even a presentation with the help of the body of specific asanas in order to describe given aspects of performing specific body movements. The transfer of embodied knowledge is very problematic as it undergoes different contextual restrictions, for example, the experience of the addresser and receiver with given body movements, possibility of verbalizing given feelings of the body, that is, language competencies to passing certain core knowledge, here, embodied knowledge. General obstacles in the intersubjective transfer of knowledge are very individualized feelings of the body among the yoga practitioners because of their initial experience with the body, its built and acquired restrictions, and advancement in practice in performing in a given moment, given asanas. It results in very varied feelings, often not concurrent with each other in case of different people; what for some is pain or physical discomfort, for others can be a natural state and even pleasure. These last definitions have rather symbolic character and come from the core of knowledge related with yoga transferred to the practitioners in literature and directly by yoga teachers.

Knowledge acquired during classes has an embodied and situated character. It is found on the level of the body and it can be renewed there and felt again. Above that, the space and time contexts of its performing are very important. This knowledge is usually connected with the classes where you work on your body or given places, parts of the day when you practice yoga at home (compare Pagis 2010a: 471-472, 484). Intersubjectivity is based on body experiences. The interviewees often said that the interviewer should try hatha-yoga practice to check the feelings and experiences. The words cannot describe the experience of hatha-yoga practice explicitly. Similar findings could be found also in other practices as vipassana meditation (Pagis 2010b: 322). 


\subsection{The method of studying corporality with the usage of visual techniques}

To the researching method called here "movie-elicited interviews" six training films from YouTube were chosen. The reason for choosing these movies from the all available websites was the ease of access by the collaborators of the researcher who were doing the interviews, could at any moment download a film from the Internet or play it directly from the computer while interviewing, and easy access for yoga practitioners. The researcher chose the films based on the analysis of such films presented on the Internet. These films can differ in case of, for example, level of professionalism in showing and instructing how to do some asanas. Three films, according to him, were done according to the rules of professional training set on perfect, close to ideal performances of asanas. ${ }^{4}$ And, three training films a bit away from the perfect training and classical yoga towards partnership yoga, and showed more naturally as it is in real class. ${ }^{5}$ We present also some picture of the author that perform some asanas and forms of visual presentations, which are analyzed in the text.

These films come from a professional DVD for beginning adepts of yoga, which fragments were put on YouTube. The professionalism of training was later confirmed in the research by experienced yoga practitioners. Here is an example comment of one of the researched about the professional films, in this case, film Utthita-parsvakonasana:

- What do you think about this film?

- This film is a pirate version of a training DVD done by professional yogis. So, absolutely, I have no bad comments to the explanation as to showing this whole asana, whole exercise. The attention was also placed on such key, right? ...what needs to be moved, which way. In my opinion, a very well-done film, very good training for someone who has never trained or trained and knows something more or less. (movie-elicited interview with a woman practicing yoga for about 5 years)

${ }^{4}$ See:Asana-Suptavirasana, http://www.youtube.com/watch?v=2uf2yTj2kK4\&feature =related; Asana - Utkatasana, http://pl.youtube.com/watch?v=gq0tBR1Jmds\&feature =related; Asana - Utthita-parsvakonasana, http://www.youtube.com/watch?v=a6CSfLbCPg (accessed: 23.07.2014).

${ }^{5}$ See: Asana - Parivrtta Marichyasana, http://www.youtube.com/watch?v=M5SwUde3X8\&NR=1; Asana - Transition to Standing Pose in Partner Yoga, http://www.youtube. com/watch?v=ppPPtcpv0kI; Parsvottanasana Pose in Partner Yoga, http://www.youtube. com/watch?v=h9obeOxoJbk (accessed: 23.07.2014). 
These films I call appropriately professional and natural. The researched, sometimes advanced in yoga, from the very beginning could see the difference in the showed films between professional yoga and professional training and natural training, sometimes calling it unprofessional. Their feelings were agreeing with the feelings of the researcher who at the moment of choosing the films practiced yoga for about 1.5 years (almost every day at home, at least twice a week under the eye of an instructor, one week long trip to yoga practice in the mountains where asanas and pranayamas were practiced 7 hours a day and, two three-day-long trips to all day exercising of asanas plus pranayama exercising) and he based his own experiences in defining professional execution of asanas and also their presentation in the films. Films acknowledged by the researcher as professional presentations were not negatively, even once in a technical sense, evaluated by the researched. However, in case of films regarded as natural, they were seen as such:

- What do you think about the film?

- Hmm, I liked it more than the previous ones because it resembled more such normal, classical classes rather than watching some soulless training tape. (movie-elicited interview with a woman practicing yoga for 5 years, Asana - Parivrtta Marchyasana)

Or they were directly called unprofessional or they were treated ironically:

- So now let's move to the last film. What do you think about it?

- Hmm... it was Parivritta Marchyasana... I liked that film because there was a very nice girl in it. (movie-elicited interview based on the video film, Asana - Parivritta Marchyasana)

Both ladies that in the beginning were sitting with their legs crossed that we call in yoga svastikasana. And now they are doing something that is completely new for them, I would not call it any asana, the more that both ladies do it differently. I do not know what that was. Maybe it was supposed to strengthen their thighs, but for sure it is not any asana known to me. (movie-elicited interview with practitioner for 8 years, Transition to Standing Pose in Partner Yoga)

During the interviews the researched were shown three films, two professional and one unprofessional, although there was also one interview with a show of six films. The researched persons were asked the following questions concerning the films shown (every film separately):

1. What do you think about the film?

2. What is felt while performing a given asana? 
3. What is the most important in a given asana?

4. When can a given asana be done completely correct?

5. How should one breathe while doing a given asana?

6. What are the consequences of doing a given asana? (the results for the body, psyche, and health)

Above mentioned questions were constructed based on a participant observation where the researcher directly took part in practicing yoga under the eye of a coach/coaches, and previously done unstructured and narrative interviews and done and analyzed photographs of asana practice. Hatha-yoga is a practice very closely related with physical exercises and the influence on the body. If it is so, what happens with the body and the work on the body should be described accurately and in detail. Techniques based only on the verbal transmission, like unstructured and narrative interviews, and even photographs and the analysis of the photographs, did not fully show the problem of experiencing the body and the results of the work on it for itself and the psyche. The practice of asanas is concentrated on the here and now, it is very difficult after you leave outside the exercise room to verbally transfer the feeling connected with practicing asanas. There can be a lot said about the effects of practicing yoga in general and about the meanings ascribed to it, but concrete and individual bodily feelings are extremely difficult to be verbally conveyed. The analysis of the training films itself and the recordings of practical yoga classes and the photographs were not enough to describe and analyze the feelings of the body. For this reason, there was an idea in the middle of the research to do the movie-elicited interviews. It was assumed that precise showing of doing some postures will allow through the help of the picture the activation of the knowledge of a corporal character and convey it in some verbal way. The film was shown to the researched without any sound, they only saw the picture, and after each film they were asked a set of questions.

The questions were used to get from the researched:

1. An evaluation of the film (as professional or not, q. 1).

2. Acquiring descriptions of feelings connected with performing the asanas, that is, descriptions of experiencing body by doing certain positions (q. 2).

3. Activating and transferring knowledge about concrete body movements - acquiring the descriptions as evidence of possessing certain technical knowledge (q. 3 and 4).

4. Acquiring the assessment of these movements in the context of performing the whole asana (q. 4). 
5. Acquiring the problem of coordination of the movements and breathing for a harmonious and correct performance of asana (q. 5).

6. Also, acquiring the knowledge about the effects that doing a certain asana has for the body and health (q. 6).

The answers to the sixth question was compared with the interpretation of the results of performing a given asana by an expert from professional films who starred in certain films and he talked about the results in the end of the movie.

In the research, unstructured interviews were used, and already in the interviews the interlocutors mention that talking about yoga, transfer of knowledge about gestures and posture of the body is not possible with the usage of the words. Although words are also needed for such a transfer to happen, conceptual knowledge about the practice can disappear when somebody is a competent practitioner of asanas, and then there is the embodied knowledge created (compare Pagis 2010a: 487). Visual materials give us more new information to the verbal reports. Pictures of practice from exercise rooms showed us what cannot always be learned from the interviews. The teacher gives instructions but also corrects using a touch, pressing or stretching body parts so to stretch muscles or the joints of the practitioner. Photographs then give us some data and information unavailable in the verbal transfer.

Video films from real classes that were the record of natural interactions also point to a very important role in yoga practice. Its necessity is visible in teaching the beginners, especially important is the assisting of the teacher in the more difficult postures. There is also a big effort of the practitioners visible, especially the beginners. The value of the visual materials to understand the yoga practice and the work on the body seemed priceless. However, it did not give us a full knowledge about the feelings of the body during yoga practice.

\subsection{What do I see and feel when I practice? Analysis of the interviews data}

In the unstructured interviews, the interlocutors often mention that speaking about yoga, transfer of the knowledge, about gestures and body postures is not possible using words. Although words are also important for the transfer to exist: 
Madam, to be honest, yoga should not be talked about, should not be discussed. Yoga should be felt, but for this transfer to be from somewhere, this news to be, to simply teach someone, feel or even only inform. This is why we are here.

Also, I simply do not know in this moment whether I gave you important things because you can talk and talk about this and, well, madam, you feel more, simply more having to do with yoga. (unstructured interview with a retired Polish language teacher)

The practitioner points out to the experience that is difficult to describe using words.

The interviews also show that while doing yoga, there is a new kind of knowledge about yoga appearing. There is awareness of the body appearing that consists of: the awareness of work with certain muscles, paying attention to "body needs" and also its connection with the psyche, and the need to sustain the balance between the psyche and the body:

Well, and the knowing of my body has improved, I know what I am able to do, how to improve the functioning of my organism, what exercises improve the work of given parts of the body, and I realized that my psyche and mood influence greatly on my body and what to do so there would be such a harmony between my body and mind, harmony.

In general, I think that before you start exercising, you should take into the consideration the needs of your body. Sometimes the legs are tired, sometimes the head, and you need to deal with it doing different exercises. For example, when the legs are tired, then it would be better, for example, to bend forward, something delicate. Another day we will say that I will not be able to do anything, so I would use some dynamic practice, something to move my body, something energetic. You should tune yourself into your body, feel its needs. (narrative interview with practitioner for 3 years, age 22)

The transfer of knowledge called "body awareness" is, however, general, words generally known are used here, for example, tiredness, body movements, energetic practice. This language scope is commonly available knowledge about the body that is why intersubjective communication is possible here. However, we do not exactly know how this "body awareness" is possible to transfer information in the situation when it concerns a concrete situation and context of performing given exercises here and now. This process of transfer of embodied knowledge seems to be more possible when using visual aids, when some reconstruction of the sequence of movements is possible and feelings connected with that that occurred in the past. 


\subsubsection{What do I see when somebody else practices? The analysis of films from the Internet used for interviews}

Films about yoga, including training films, can be analyzed independently from their later usage in the interviews. I show their analysis in order to later understand better their interpretations by the participants of the social world of yoga practice. Before we use a film in the interview, it should be earlier analyzed to reconstruct the meanings in them and show their formal structure of presenting a given subject.

In chosen films we have two types of conveying the embodied knowledge, natural and professional way:

1. Natural way, straying away from the "drawn" ideal (such that we can show in a drawing disregarding the possibilities of the body), closer to natural, averagely done asanas. Sometimes exercises in pairs are shown. Exercises in pairs stray away from the individually done classical yoga that is strictly individual practice, although its effects can have, should have positive psycho-social meaning. The verbal training in the films, during the exercises, is connected with the movements of the body and accompanying breaths that are supposed to happen in a proper moment. Sometimes, there are metaphoric comments describing particular body movements: "And I am really engaging my core and twisting from the center to create space and freedom inside the body as well as out" (see: natural presentation of asana - Parivrtta Marichyasana ${ }^{6}$ and natural presentation of asana - Partner Yoga Guide: Transition to Standing Pose in Partner $\left.\mathrm{Yoga}^{7}\right)$.

In yoga practiced with a partner, as in the above exercise (Transition to Standing Pose in Partner Yoga), the coach comments the movements of the body that both people have to do to move on to sitting in the sukasana position from the standing position. She also talks about that that there is trust appearing when two people have to lean against each other with their backs: "It is a trust issue and it takes a lot of practice sometimes." There is also a comment about interpreting the feeling of the position and the body: "It's great fun between the transition from the floor to standing or from standing to the floor" (see: natural presentation - Parsvottanasana Pose in Partner Yoga - pyramid pose ${ }^{8}$ ).

${ }^{6}$ See: http://www.youtube.com/watch?v=M5SwU-de3X8\&NR=1 (accessed: 8.03.2009).

${ }^{7}$ See: http://www.youtube.com/watch?v=ppPPtcpv0kI (accessed: 8.03.2009).

${ }^{8}$ See: http://www.youtube.com/watch?v=h9obeOxoJbk (accessed: 8.03.2009). 
Before performing the above posture (pyramid pose), the instructor talks about the benefits from doing this asana. Next, she explains particular movements and their coordination with the breath. In the above moment in the picture she is saying: "feeling the nice back," and next she also points to feeling the hamstrings stretch. The instructor somehow gives points about feeling the body, that is, what the practitioner should feel in a given moment.

2. The professional way of presenting asanas, close to the "drawn" ideal, showing in a technical way, from different points of view, step by step movements with the accompanying verbal coaching on how to perform a given asana. At the end there is a statement about the consequences of practicing given asana for the body and the health. Mentioned are only the consequences of performing a given asana for the body and alternatively the psyche, however, there is nothing said about what in a given moment is felt by the body or the mind, or mind and the body. The arrows showing the direction of movements or body stretching and lines showing the position of particular body parts acquire visualization of postures. On one picture showing movement are also close ups of legs and hands that are important to show the correctness of doing given poses. There are also views shown of a given asana from different captions, for example, from the side and top to visualize the whole position. In professional presentations, there is no suggesting of body feelings, all the time with the technical support, with the emotions discarded from the language specific movements are described and they are shown in more detail with the usage of the picture (see visualization of the posture with the usage of lines and arrows at fig. 5-6: Utkatasana - chair pose called also a fierce pose ${ }^{9}$ ).

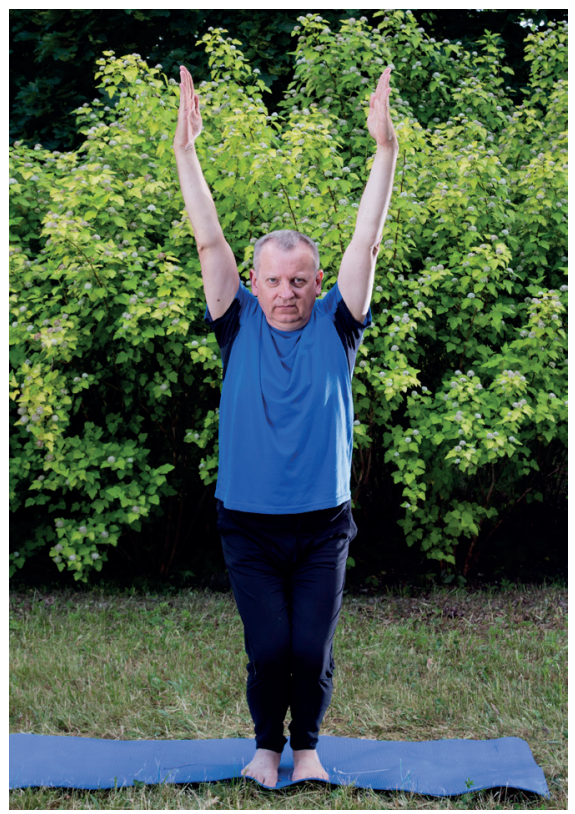

Fig. 5. Utkatasana - fierce pose called also chair pose (photo by Kamil Głowacki)

\footnotetext{
${ }^{9}$ See: http://www.youtube.com/watch?v=gq0tBR1Jmds (accessed: 8.03.2009).
} 


\section{CHAPTER 5}

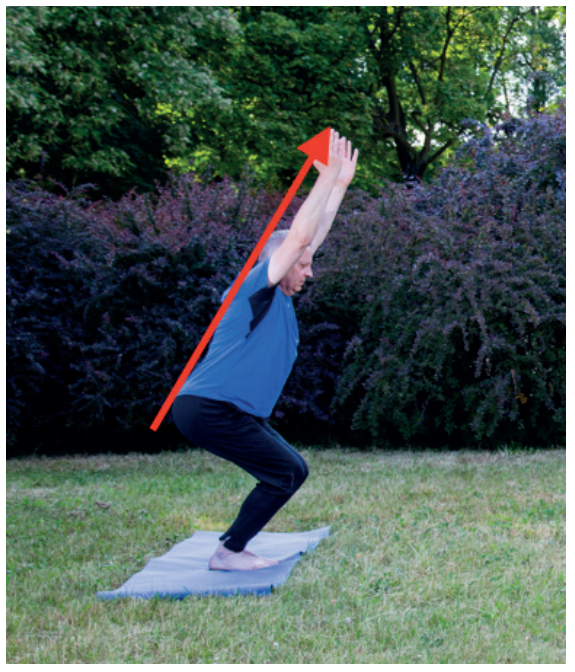

Fig. 6. Utkatasana - fierce pose called also chair pose (photo by Kamil Głowacki)

We could see also visualization of the position with the usage of lines and arrows and close ups of body parts, different captions (see fig. 7-9: Virksasana - tree pose). ${ }^{10}$

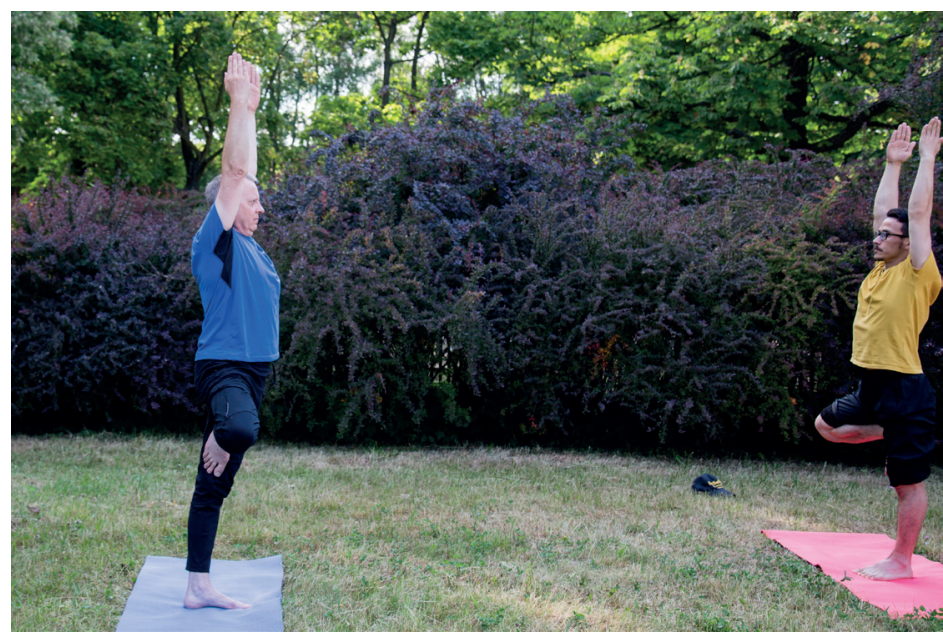

Fig. 7. Virksasana - tree pose, picture of the author and student of "Meditation for managers" class (photo by Kamil Głowacki)

10 See: http://www.youtube.com/watch?v=9JXcFeafy2k\&feature=related (accessed: 8.03.2009). 


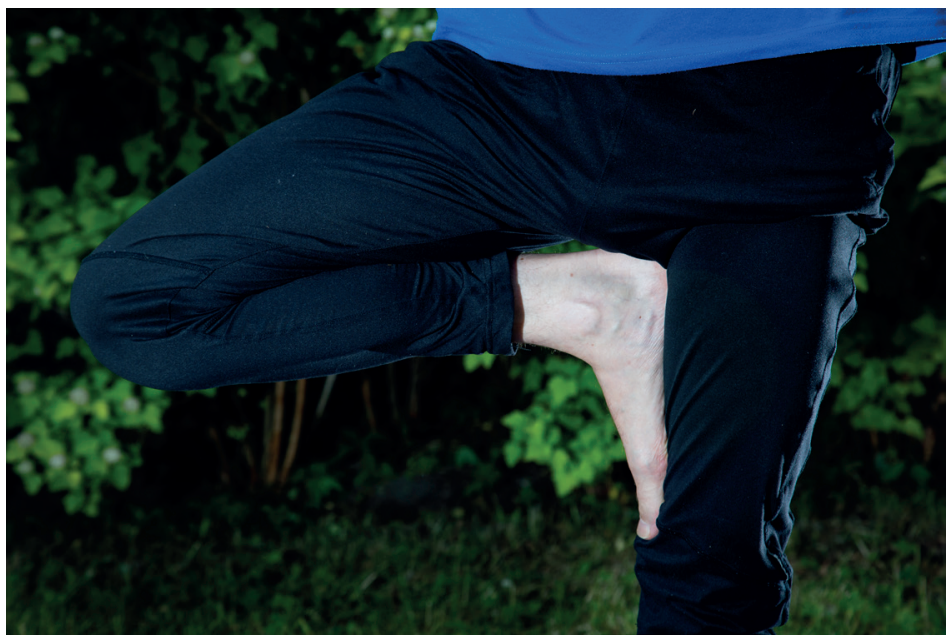

Fig. 8. Virksasana - tree pose, close up (photo by Kamil Głowacki)

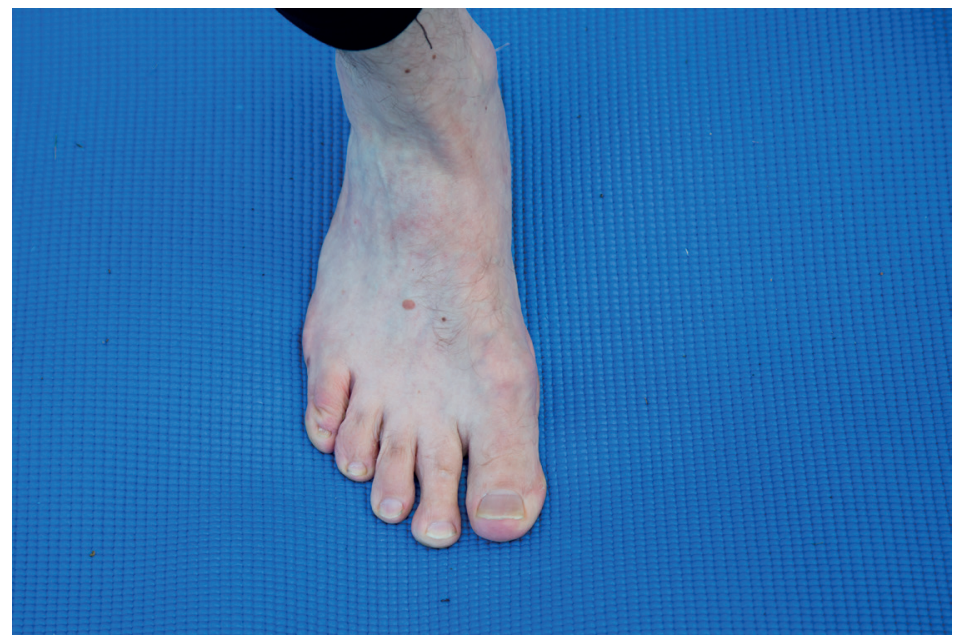

Fig. 9. Virksasana - tree pose, close up of feet (photo by Kamil Głowacki)

Such visualization is supposed to help in the transfer as to how the posture should look, and also substitutes as if a literary way of showing experiencing the body in this posture (emotion transfer, physical, psychic, and spiritual feelings, etc.). The narration describes entering a posture and exiting the posture, and particular movements and their coordination with the breath that should be calm all the time. In the end effects of a posture are shown, in this case, this is strengthening leg muscles and feeling of physical and psychic balance. 
View from above shows how the pose should be done properly (see the movie quoted above). The outer look is equal here with the proper performance. The words only help the pictures, accompany them. The key here is the visual presentation in the knowledge transfer about the gestures and the body (compare Schnettler 2008: 129).
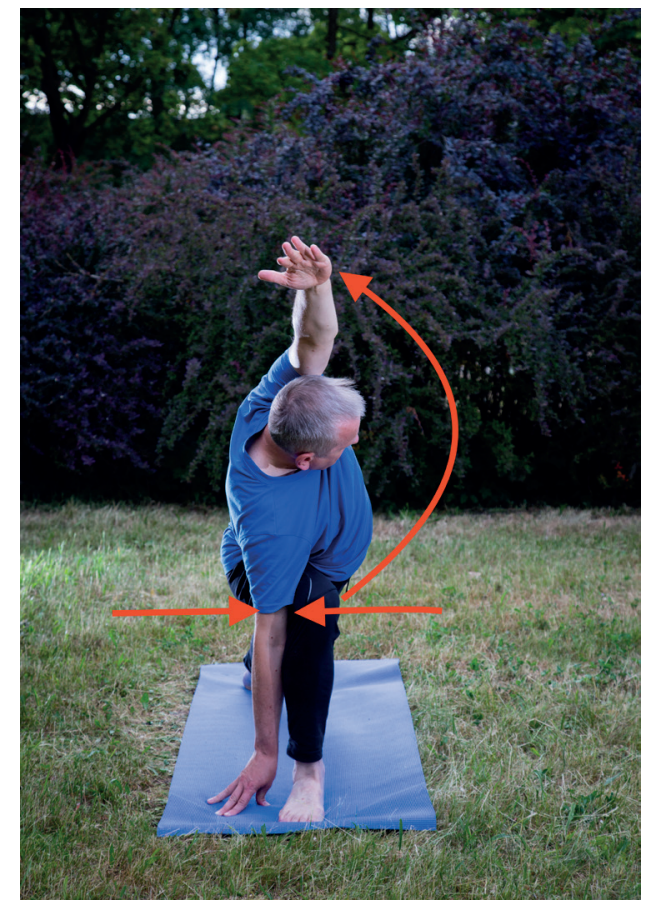

Fig. 10. Utthita parsvakonasana, the extended side-angle pose (photo by Kamil Głowacki)

In the film Utthita Parsvakonasana - Yoga for Beginners ${ }^{11}$ there is a precise and technical description of entering the pose. The arrows are helping to show the direction of the work of the body parts and their necessary direction of pulling out (fig. 10-11). The pose is described by recalling the knowledge about other asanas that are the indirect steps to enter given asana. Thus, the knowledge transfer about more difficult and complicated asanas is done based on the guidelines of possessed knowledge about the basic asanas. We thus start here from tadasana (mountain pose) and enter utthita hasta padasana (star pose), next you need to move to set the feet as to the second warrior (Virabhadrasana II), next we move to the second warrior, and only then we slowly move, doing few movements, to utthitaparvokonasana pose. Everything is, however, supported with a picture of movements, thus the knowledge is transferred directly to the film recipients.

Different captions are trying to show more or less in a versatile way how the body works (fig. 12-15). Lines and arrows show the correct body positioning and specific angles, for example, right angle in the left knee and the straight line link the foot from the right leg with the extended right hand.

\footnotetext{
11 See: https://www.youtube.com/watch?v=a6CSfLbC-Pg (accessed: 19.06.2015).
} 
Elements very vital for the safety of the pose performance are underlined with the red, blinking exclamation mark. The exclamation mark is an international mark pointing to something important appearing, and the red color is a warning color.

The caption shows the positioning of the legs and knees in the initial phases of entering the pose. It is started from the sit in virasana, thus there is here the recollection to the knowledge about other asanas; we see a cumulative way of building embodied knowledge.

The lines show the necessity of symmetric positioning of the body in asana: parallel stretched arms, knees close to each other, parallel thighs. It is pointed out verbally to the free breathing and relaxed face, these two indications concern almost all asanas.

In situations when it is not possible to perform a full pose, you can for the sake of easiness use some help: bolster and blanket. $^{12}$

${ }^{12}$ Foranalyzed reclined heropose, see: http://www.youtube.com/watch ?v=2uf2yTj2kK4 (accessed: 1.03.2012).

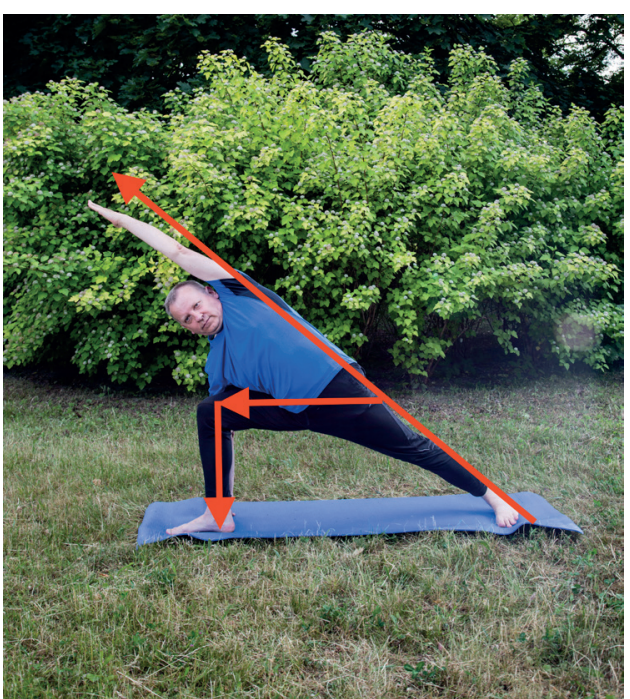

Fig. 11. Utthita parsvakonasana, the extended side-angle pose (photo by Kamil Głowacki)

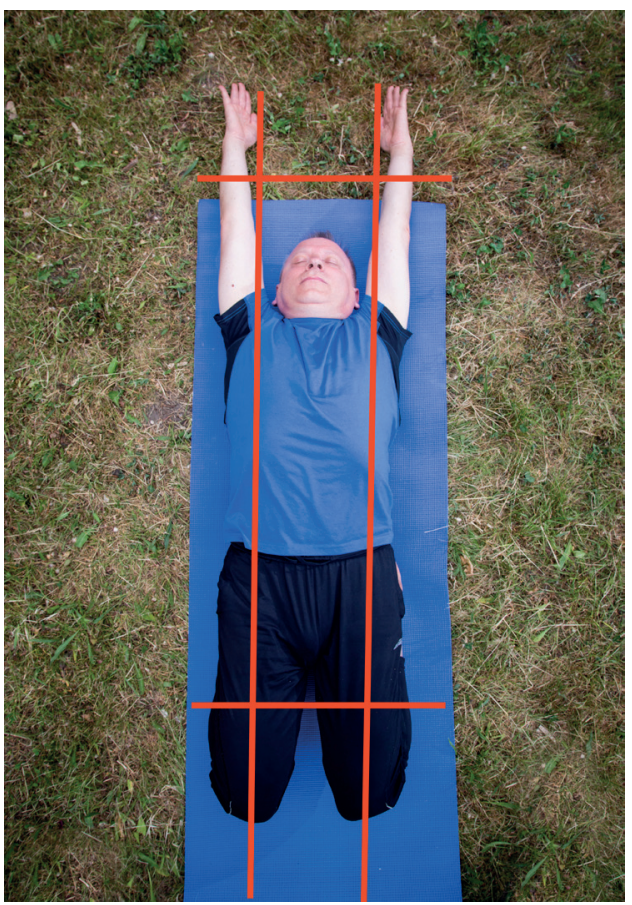

Fig. 12. Supta virasana, view from above (photo by Kamil Głowacki) 


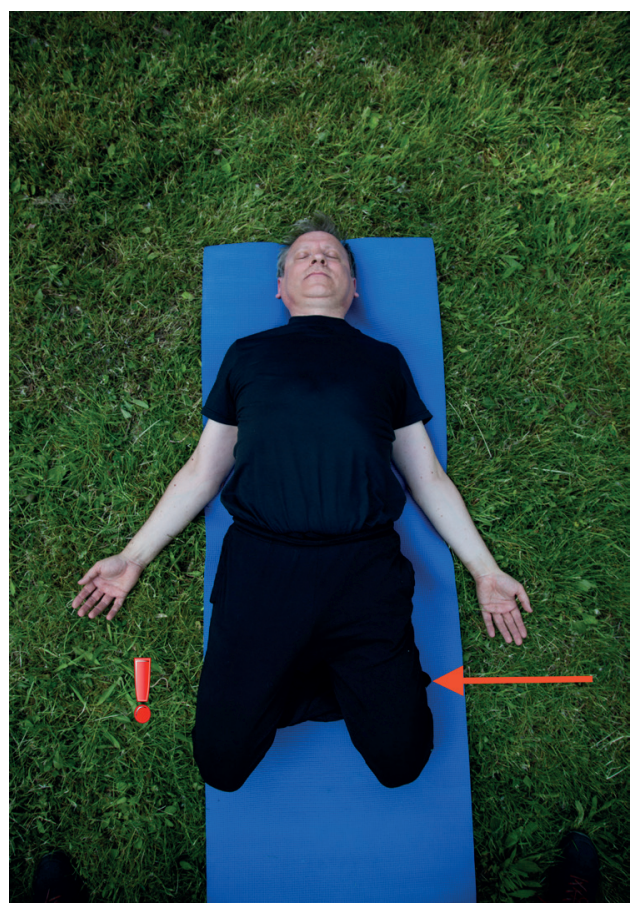

Fig. 13. Supta virasana done with help of accessories (blanket and exclamation mark) (photo by Kamil Głowacki)
In the beginning of supta virasana there is a warning appearing and a blinking exclamation mark and a verbal comment saying that the position should be done with extra care in case of problems with back and knees. "In this situation, you can do the exercise only after consulting a yoga teacher." The red, blinking exclamation mark here is as an attention sign. In the instructions, there is often a suggestion to relax the face (in the instruction to the above asana there is such as well). It is very important as in many postures, also in supta virasana, one can be tensed even though the body is stretched. The relaxation of the face and free breathing somehow relaxes the whole body, and can also be an initiative movement that relaxes the body. The instructor here says that the "top of the thighs stretch in this posture, keep peace in the head." It points to the fact that there is some tension and fear while performing a given asana.

The transfer of an embodied knowledge with the usage of visualization and the verbal help shows
Fig. 14. Supta virasana - close up to the knees (photo by Kamil Głowacki) 
us only the outer layer of the embodied knowledge. There is almost no mentioning of experiencing the body, feelings, and experiences. Only in natural presentations do we have, although scarce, just as it is during real class when the instructors often point to certain feelings, suggestions and interpretations of the feelings (e.g., pleasant pain from stretching) and understanding the body in relation to the space (feel the space in your own body). That is why there came up a need to use a different research technique in order to gain data concerning the experience of corporeality in yoga practice, such a technique is a movie-elicited interview.

\subsubsection{What do I feel when I practice? Analysis of the movie-elicited interviews}

The interlocutors doing yoga were definitely more eager and concrete in talking about their feelings connected with body functioning during the movie-elicited interviews than in the same but unstructured and narrative interviews. Films make their empathy work, allow recollecting the way the body and the psyche work in case of doing a given asana, and not only generally speak about the way yoga influences the body. The unstructured and narrative interviews activate general knowledge about the influence of yoga on the body and the psyche, and possibly on the spirit. The knowledge about the body is also conveyed in them, however, it has a general character and is expressed in a spoken language, and that is why it is also intersubjectively available.

Training films about the practice of specific asanas allow the interlocutors to concentrate mostly on the body and its feelings, showing specific movements in asanas allows for a mnemonic reconstruction of these movements in relation to the body and "subjective" feelings concerning given exercises, both physical and emotional, and even spiritual. Their narration is being placed directly in a given exercise and not only in the discourse connected with the story about the motives of taking up yoga, its meanings and common definitions, and general consequences of practicing yoga for the practitioners.

\subsubsection{Non-passable knowledge. Difficulties with verbal interpretations and description of asanas}

Some of the practitioners, also in the movies-elicited interviews, do not talk about the physical experience that one experiences while performing asanas, they claim that one needs to experience them personally suggesting that a verbal description of feelings is not possible: 
- Please, tell me what do you feel while doing asanas, how does the breath behave?

- Well, here you can see supta virasana. Well, you need to practice this to (laughter) understand it. What else is there to say? That's it. Well, there is tension, stretching, down we stretch the muscles, shown in such a pose, in general to relax.

- And what does doing this specific asana give?

- It cannot be described just like that, it needs to be experienced. (movie-elicited interview with yoga instructor)

Some practitioners have difficulties with a verbal interpretation and the description of asanas. When asked about the feelings concerning performing a given posture, the asked persons either did not want to say a thing or avoided the answer:

- And what do you feel when practicing this asana?

- I don't know because when you go to this school that I went to... I practiced at, this posture is done slightly different.

Experiencing the body is a very personal and individual feeling. Even the visualization of a given gesture or pose is not able, for some, to turn on the verbalized form of empathy enough to express in the spoken language what is felt in the body in the moment of performing a pose. It is important to do certain moves in a certain sequence. Feelings are somewhat movements and not "emotional feelings" that can be expressed in a language. The language seems to be some sort of an edge of expressing the experiences connected with gestures of the body in yoga. Experiencing the pose is encompassed in its performance. The interlocutor answers very generally to the question about the consequences of practicing a given pose:

- What are the consequences of a given asana for the body and mind?

- It is one of the relaxing asanas. I mean, it does not cause tension but relaxation. (movie-elicited interview, Parivrtta Marichyasana)

The interlocutor assumes that the words relaxation, laxity of muscles are generally understood in an identical way by others, which enables the agreement about the consequences of performing a given asana, what is more, the interlocutor says that the feeling of relaxation is the denial of tension. We do not have a too tightly set of corporal or psychic feelings, but a general reference to a given feeling of "relaxation," what for intersubjective agreement in this conversation is enough.

In a different case, the practitioner (age 45) is unable to pass on the knowledge about experience of a yoga pose (utkatasana), the knowledge seems to be in the same individual performance of the action: 
- And what is felt while performing this asana?

- I do not know. I did this exercise. It is familiar to me but I do not remember whether there were any reflections of emotions connected with its practicing. I guess only focusing on the action. (movie-elicited interview, Utkatasana)

When it comes to the consequences, then the same interlocutor gives only one interpretation that utkatasana (chair pose/fierce pose) is a stretching exercise, however, according to experts (see the film), this is an exercise energizing the body, strengthening the ankles and appropriate development of the leg muscles, development of the chest and toning of vital organs. The interpretation of the interlocutor here is not coherent with the interpretation of the experts. It can state that the knowledge about the consequences of practicing yoga is acquired in the process of socialization and from the experts' statements.

Above that, the interlocutor stated that it is difficult to talk about physical experiences while practicing yoga. She encouraged the researcher to start such exercises to understand what she was talking about. Again, it is visible here that what turned out to be very difficult is the verbal conveyance of the knowledge about the corporal experience of given yoga postures.

The experience of the practice seems to be strongly individualized. It is seen in the different from expert interpretations concerning the consequences of practicing given asanas (e.g., health benefits) appearing also in other interviews. Yoga practitioners create the knowledge about practicing yoga for their own usage, yoga can help them in different aspects, in their opinion, and it does not have to always agree with the opinion of the experts. Some part of this knowledge can, of course, come from the experts. Some research confirms the opinion of the experts about given asanas and how they work on specific body parts. This knowledge is probably taken from the teachers and widely accessible publications about it.

It needs to be underlined that experienced practitioners also talk about problems with the verbal transfer of the experience of yoga practice. They emphasize the need to experience the practice and having some reflection on this basis in order to understand yoga practice:

The respondent had a problem with describing the emotions, "dressing in words" what happens to the body and mind while performing given asanas. As she said after the interview, "it cannot be described simply." Only a practicing person and having its awareness is able to understand the sense of the practice. (movie-elicited interview with practitioner for 8 years; it is a description of the situation of the interview) 


\subsubsection{Body empathy based on visualization}

Through the analysis of the data obtained by the movie-elicited interview there was a new category generated, called "body empathy." It is activated in the researched persons by the usage of the pictures of corporal practice. Body empathy concerns several important aspects of skills of recognizing body feelings:

1. Description of specific technical movements in asana, their relation with each other, and the meaning of their importance for performing a given asana.

2. Recognition/remembering body feelings.

3. Recognizing emotional feelings while performing given poses.

Empathy of the body allows the describing of and conveying to others the corporal knowledge, in this case, it is a skill of performing specific movements of the body in doing hatha-yoga.

On the basis of the analysis of data obtained from the movie-elicited interviews, I created two hypotheses that I will describe and justify in a further analysis of these interviews:

1. Body empathy can be activated by visualization of body gestures.

2. "Body empathy" is very important in the transfer of embodied knowledge.

Describing a given asana is connected with verbal presentation of particular movements in asana and its relation to each other and sometimes the way of breathing. Experienced practitioners in a visible way better and more analytically describe asanas shown in the films from less experienced ones:

- Just like with the previous film, I would like to ask for a comment.

- The instructor starts every asana from a correct posture, that is, from the tadasana pose. Now, he moves on to the asana that we call utkatasana. It is an asana rather full of effort and here you need strong thigh muscles. It is important to pay attention to this that the knees were not in front of the toes. To be honest, it would be easier to do this asana if somebody would push the knees in front of the toes, however, then it is not an asana and it is done incorrectly. It is important to stretch from the tail bone to the tip of the fingers of both hands. (movie-elicited interview with practitioner for 8 years, Utkatasana)

Body empathy here is recognizable by a precise description of actions of particular body parts and it is about a precise connection of the picture and given pose of the body with the feeling of your own body as if you were performing a given position in a given moment when you watch the picture and comment on it verbally. Body empathy rises with 
the experience in practicing yoga as we see it in the example of the film description done by an experienced practitioner:

\begin{abstract}
Now, we observe parsvakonasana, that is, at least what I think. In general, it is shown rather as a show. However, ladies started well here, I mean, they have a good distance between their feet. Both feet are placed in a right angle and this asana's aim is to stretch the leg muscles and also spine. It is important here for the hips to be at the same height above the ground and parallel. It is important that the thighs were strong, and in this case if we were doing parsvakonasana with the right leg to the front, then it is vital for the right calf to send itself to us and we push ourselves away from the sole of our right foot, and, to be honest, from the bottom of our big toe of the right foot and we stretch outside, the pushing away happens to the outer side of the right groin but the weight should not, the weight should not be kept on the right foot, but so both feet were active, that is the left foot in this moment is strong and the outer left rim of the left foot pushes against the floor, the left thigh turns inside and the calf to inside to achieve the pushing of the outer rim. (movie-elicited interview with practitioner for 8 years, Parsvottanasana Pose in Partner Yoga)
\end{abstract}

Experienced practitioners are able to precisely describe the poses and analyze them in detail in the context of adjusting particular body parts towards each other in a given pose. In the poses Partner Yoga, for example, they can see the lack of movement and coordination of their partners (the interlocutor declines describing the status of yoga exercises). Yoga is for her an individual practice:

And their knees are then in front of the toes. But, the beginning is good because the outer rims of the feet are adjusted parellelly, but no, the knees do not stick out. We can only see here that the lady in grey trousers has her legs constantly straight, however, the lady in black trousers is not able to straighten her legs. We do not even know where this is heading. This is not any known asana to me. I doubt that it could even be an asana because asanas are done individually. Asana is simply a position in Polish language and what the ladies are doing here cannot be done individually. (movie-elicited interview with practitioner for 8 years, Transition in Standing Pose in Partner Yoga)

We can see a lack of coordination in the moves, it looks as natural presentation $^{13}$.

The marking of the importance of particular movements concerns the meaning of particular movements through a correct performance of asana. The meaning was ascribed to the movements by the practitioners based on the question that was asked by the researchers while showing the film.

${ }^{13}$ See film: Partner Yoga Guide: Transition to Standing Pose in Partner Yoga: https:// www.youtube.com/watch?v=ppPPtcpv0kI. Retrieved 19.06.2015. 
The question about what is the most important while performing a given asana was supposed to inspire the interlocutors to describe movements and their relations in a given asana, this question also tested their knowledge about the given asana:

- That means... what is the most important in a given asana?

- What is the most important here? Yes, this bending here... bending of the knees at the right angle. The knee would equal the feet so it would not go... with feet so it would not go forward. It means that there needs to be a right angle when it comes to the hand. You also need to pull in the stomach, the bridge a bit forward... And, not the other way around so the stomach went forward... Well, that is all, I think. And what is also important here that you need to breathe all the time. (movie-elicited interview, pose Utthita Parsvokonasana)

It was often answered to the question about a given asana that the coordination of the whole body is crucial, that, in reality, in every asana the whole body works. It was repeated many times that there are no most important movements, all of the movements from a given asana are important plus the breath. Also, the coordination of all body parts used in the asana is important:

- And what is the most important in this asana and what describes it?

- I think that here even though this is a standing pose and in general we work here more with the upper body, but we, however, need to have strong legs to hold them properly. The breath in asanas should be calm, it must not be stopped. (movie-elicited interview with a practitioner for a year, Utkatasana)

The coordination of the body movements with the breath is an additional stage of skills that needs to be acquired to perform correctly given asanas. Very important thus is the knowledge about the technique of breathing. The breath in should be done in poses that cause raising part of the body, however, the breath out in poses when some parts of the body go down. However, with the locking of a pose, the breath should be calm and even. This is a difficult task because the organism gets tired and somehow automatically speeds up the breath:

Rather, as I have mentioned earlier to you, the breath should be first and foremost deep, calm, and harmonized with the body movement. There are, however, passages that, for example, require stopping the breath for a moment, but this concerns only changing of the poses from one to another. However, well, that is about that that the breath in asanas look like... The main rule is that if the movement is up, then it is assisted by a breath in. In the moment when a part of the body is going down, whether the hands are going down or the torso, then this is happening on 
a deep breath out. And at the moment of holding, keeping the pose you just simply breathe evenly and calmly, you should breathe in such a way that this breath does not cause effort, should be calm which is not that easy because the body tires, undergoes physical tiredness and there appears a tendency to shorten the breath. (movie-elicited interview, Utkatasana)

The empathy of the body is also connected with the need to coordinate the psyche and the body. Exercises are also done by our psyche. And for a full correctness to perform given asanas psychic work is needed:

- And when is it possible to do the whole asana correctly?

- A great influence on that has firstly the physical capabilities of a given person, the state of health, the work that you do... the sole fitness has a great importance here, also psychic predispositions because, frankly speaking, while doing such asana, you feel both tension and relaxation... and with the mind we need to eliminate what hurts us, we need to hold on, stretch the pose, we need to feel rather comfortably in it. It needs to go together these physical predispositions and this psychic strength, to not do anything too fast that later on will bring us no effects. (movie-elicited interview with practitioner for a year, Utkatasana)

The correctness of performing a given asana is also associated, according to the interlocutor, with the recognition of own possibilities and needs of the body:

There are many elements influencing the performance of a given asana and it seems to me that you can always do it correctly. It does not have to be... you do not need to do it like, for example, the man in the film is showing, but the correctness, I believe, that you can always do it according to your possibilities. (movie-elicited interview with practitioner for a year, Utkatasana)

Her definition of performing the asana is not coming closer to the ideal, but performing the asana according to the possibilities of their own body and the psyche. Asana can also be done correctly with the usage of bolsters and blankets. The most important thing here is to recognize your own body:

- And when can you do this asana completely correct?

- Well, I think when we have no reluctance when it comes to leaning back. I, for example, never hurry with fully completing such asana... so it would be from the very beginning to the very end so perfect if somebody who practices it for years does it because I am not able to do that. However, I think that if we do not force ourselves to too much and demand from ourselves too much, we can do this pose; we can do it. Even if it is with some help, it can be done; it is difficult to say, well, after half a year? It also depends on the physical conditions of a given person. (movie-elicited interview with practitioner for a year, Supta Virasana) 
The correctness of performing some asanas is a question of interpretation and point of reference, even negotiation. For a correct performance of an asana, there is also needed coaching from the teacher:

- When can this asana be done completely correct?

- What does it mean, completely correct? This is such a doubt that I would have in general with this question because, to be honest, the man at home is not able to do independently and correctly any asana unless he is an experienced yogi, practices for many years, and is a teacher, then he is able to do it independently. But, still somebody with a higher degree will come and correct him, push him, turn him the right direction so here to do it correctly you need to be in class with an experienced teacher that would adjust you as much as you can so the asana would be done correctly according to your possibilities. (movie-elicited interview with practitioner for 5 years, Utthita Parsvakonasana)

Some of the interlocutors remember in details what was happening with their bodies while performing asanas. The film is only "triggering the memory" here:

In general, this whole pose is like this because, well, it gives more verve to this pose, it is such an energizing pose... you can really nice... at least I feel it so... I simply felt an air flow through my whole body. It was so... I felt aired everywhere from the tips of my fingers to the tips of my toes. (movie-elicited interview, Utkatasana)

Often, during such watching of asana poses in a film, the practitioners are able to interpret the feelings of the body based on what they see in the film. The empathy is being started by the visualization of the poses. Even if the practitioner has not done the position, then his/her corporal empathy is developed as in the below utkatasana (chair pose) that the quoted practitioner did not have in his repertoire of everyday exercises but was able to foresee the pain in the knees:

Such stretching, for your back, for sure, it causes some tension in the thighs, in the legs, in the knees. If you continue on like this for longer your knees could hurt. (movie-elicited interview, Utkatasana)

The practitioners seeing the film picturing some exercise are able to describe and even foresee the reaction of the body, especially when they have more experience in performing a given pose. Their knowledge is somehow in their bodies, but the picture enables its activation. The more the experience of the practitioners in yoga practice is, the more awareness of the body and empathy. Here is a detailed description of "the work of the body" while watching the Supta Virasana asana: 
- If before going to bed we do such an asana, then we could literally eat and lay down in this asana. We do not have to wait. This all will digest itself because, yes, we adjust the pelvis in this pose between the calves, feet are on the sides of the body, that means that the pelvis is nicely opened, the stomach tenses nicely, the sides of the body stretch, the whole torso elongates, thus, there is such capacity for flow in the intestines and this pushes and this helps in the digestion. Not only that this pose actually works cool on such things after eating but also thanks to it we can remove rheumatic pains in the knees even though there is a strong tension on the knees.

- And what do you feel while performing this asana?

- Me, for example, here I will have to again support myself with what I have to help myself in this exercise. Of course, using different bolsters, pillows, or a blanket. However, I feel such an even stretch on the sides of the body and in the parts of the pelvis more or less up to the sternum, that is, all the inner organs. And I feel my thighs very strongly, the front parts of the thighs just simply with a stronger tilt I really feel this tension. (movie-elicited interview, Supta Virasana)

Well, you can really relax in this pose, in this asana, although you have to be careful and introduce it step-by-step because the pain in the knees can be tremendous because I have it exercised somehow, but Supta Virasana... I can allow myself to lay down in it, but, nevertheless, it hurts and, of course, you have to be careful a bit while leaving the pose so to lift yourself up step-by-step leaning against on your elbows and not to get up suddenly because it can cause pain and some damage. (movie-elicited interview, Supta Virasana)

Body empathy is also connected with remembering and replaying in the mind the fact of pain or some discomfort. Some of the interlocutors feel during the exercises some discomfort or pain, the body remembers these feelings and while watching the film, the interlocutors can remember them:

Me, for example, with such poses, I often feel such a cramp in the area of the sternum because it is a strong stretch, right. It often pops me. But, it is not that it clogs me, but the inner organs that start working, they are squeezed on one side and when I feel such a thing... that such a thing is happening to me, then either I let some breath... I let go out of this whole tension, I breathe in again and deepen this pose and it is OK. (movie-elicited interview, Parivritta Marchyasana)

Some experienced practicioners saw in the above asana, done in a specific way, Marichyasana done by women during their periods:

- You know how this asana is called?

- Marichyasana, but in this type, this Marichyasana is done for women during their periods because normally this Marichyasana we do in a completely different direction. However, so there would be no pressure on the abdominals, we do asana in this way, just as I have mentioned, during the period. Normally, we do this asana so that the torso is turned about 180 degrees in the opposite way. (movie-elicited interview with practitioner for 8 years, Parrivrtta Marchyasana) 
- What do you feel when doing a given asana?

- In this case? Well, pain and lack of stability. I think that's all, well, generally, it is difficult to hold this pose and you need to work to do it correctly, which is not easy. (movie-elicited interview with practitioner for a few years [auto-definition - medium advanced], Utthita Parsvakonasana)

The memory of the pain in this asana is often eliminated in the stories with laughter:

- What do you feel doing this asana?

- Everything hurts. This is a hellishly difficult asana that is done at the beginning of the class, even before the teacher coming, there is such a rule that when the person comes, s/he adjusts, takes all the help that s/he needs, and lays in this asana to start stretching the body. However, I was never able to do this asana without help, that is, without the blocks, the yoga belt that is also very useful that you tie your knees so they would not go apart in all directions, blocks underneath your back. Everything hurts. You stretch your thighs a lot, you stretch your back, sometimes the man is even not able to put the arms behind the head, yes? I just stay in a simpler version with the hands along the torso with the palms turned. It hurts, the asana hurts (laughter). (movie-elicited interview with practitioner for approximately 5 years, Supta Virasana)

The experience of the pain and the overcoming it is sometimes the condition to gain better fitness and ability to perform given asanas. Overcoming the pain causes that we do given asanas better and there is content because of it:

- And what do you feel doing a given asana?

- As usual, pain and you fight with yourself, right, so as to last the longest in the given pose. The longer you manage, the harder it is because the muscles start to tremble because your arms and legs start hurting you. But, it is worth it. To be honest, the longer you train, the more pleasure you get from doing a given asana correctly.

- What is the most important in this asana, what describes it?

- Well, the most important is, I think, that here is just like in most or maybe in all, well, the precision, yes... The better I do it, the better it is for me, and the longer I hold it, the better for me. (movie-elicited interview with practitioner for a few years [auto-definition - medium advanced], Utkatasana)

To positive consequences of performing some asanas one can include gaining mental balance. The consequences of gaining physical balance (e.g., being in balance in a given pose) can be gaining mental balance:

- What are the consequences for the body and the mind while performing and after performing this asana? 
- Consequences for the body are such that it stretches these muscles that should be stretched. The consequence for the mind is, for example, that I gain balance and the balance in standing asanas like this was shown here as a key factor. And when you get the balance of the body, then the mind starts getting the balance, too. You start to think more the way it should be. (movie-elicited interview with practitioner for 5 years, Utkatasana)

The practitioners talk about the benefits (consequences) coming from doing given asanas. They have the knowledge about what a given asana does with the body and how it influences it. Every exercise has its aim. Uthitta Parsvakonasana also has an aim which is, according to one practitioner, broadening the chest, enlarging the capacity of the lungs, and stretching. The practitioner also uses a very general language and calls her work on the body breaking the blockages of the body, coping with own restrictions, and broadening physical possibilities (movie-elicited interview, Uthitta Parsvakonasana). This is a language going outside a given description of the exercise, it describes general processes that are more difficult to locate and specify against the places of the body. It is somehow a "meta-corporal" language because it refers to the body as a generalized being that you work on specifically to achieve more general aims, more difficult to specify in the description of behaviors. Instructors and other yoga practitioners also mention this breaking of blockages. Thus, these are intersubjectively available terms acquired during practice with the help of which you can describe your experience with yoga.

You can empathically perceive not only positive consequences of doing yoga but also negative after-effects. Sometimes the consequences of doing a given asana can be negative for the organism, it refers to, for example, situations of incorrect performance of an asana:

- And what are the consequences for the body and the mind while doing a given asana?

- If you do these asanas incorrectly, your head will ache. If you do it, for example, too fast or at some point you pull something and you will be standing in a given position, your head can really ache. (movie-elicited interview with practitioner for a few years [auto-definition - medium advanced], Utthita Parsvakonasana)

The body in the practice of asanas is often perceived as something outer. It is the body that works itself in some poses and the practitioner only observes it. The body becomes an independent subject and when it is put into a given pose, for example, Supta Virasana, it works independently: 
- What does the breath look like while performing this asana?

- In the beginning, there is usually fighting with yourself because it is always so that the man forgets what was in the previous class, that you are not supposed to fight with the breath, and starts fighting. And only in the moment when you are lying down, when the person adjusts and starts more or less to feel every muscle, how they go apart, for example, how... But, here when you lay down, you do not work, the body works for you, right. With its own weight it stretches the muscles that are to be stretched and only then the breath stabilizes, when you find some ... because in yoga you always have, in asana find such a comfortable moment for oneself, sometimes it lasts long, that is why asanas usually last two to three minutes, right.

- To adjust correctly so you would be comfortable, so you would feel good?

- So it would be comfortable, just like that. In every asana you need to find such point, you strive for that that it would be comfortable, and then the man relaxes some parts of the muscles and then it is a full feeling of this asana just as it should be. (movie-elicited interview with practitioner for 5 years, Supta Virasana)

Very important in the correct practice of an asana is correct placement of the body. Everybody finds the best position for himself to limit the discomfort. Coordination of the mind with the body is thus done all the time while performing asanas.

While doing the exercises there are also different emotional feelings, for example, "childish happiness." The film quoted shows a given pose, allows the interlocutor to remember the felt emotional states. The body empathy concerns also the skill to read the emotions appearing while doing given asanas. Here is part of an answer to the question: And what do you feel during this exercise?

I feel some relaxation, some such... something that... I like this supta virasana because my previous teacher also praised me from this pose and I did it as an example. This, there is something nice in it, like, for example, in a different pose that is about that we hung on the ladders head down just like bats. And this frees this childlike happiness in me. I just lay, the Earth can turn, and I can relax myself here in such a way, in a rather comical position. Well, this that something like this happens that I like it a lot. (movie-elicited interview with practitioner for 7 years, Supta Virasana)

\section{Visual perception and interpersonal bodily empathy}

In two films there is a problem appearing with corporal interpersonal empathy. ${ }^{14}$ Yoga is generally a practice based on the individual work, on the concentration of the individual on oneself, own body and

${ }^{14}$ See: Partner Yoga Guide: Transition to Standing Pose in Partner Yoga, http:// www.youtube.com/watch?v=ppPPtcpv0kI; Parsvottanasana Pose in Partner Yoga, http:// www.youtube.com/watch?v=h9obeOxoJbk (accessed: 23.10.2011). 
mind. The body in asanas is also "a partner" that a given individual has to communicate, understand, see needs, make it endure the discomfort longer and often accept the pain, overcome the fear (as it is in the inverted poses). In atypical situations, when certain poses are done in pairs, it is essential to use the corporal interpersonal empathy, when we have to understand not only the needs and restrictions and the possibilities of own body but also the partner's body. The corporal interpersonal empathy is essential, for example, in dancing, rock climbing where the movements of the partner taking part together in these actions are foreseeable, defined by the other partner. The coordination with the partner is essential to perform a given task that a given body is engaged with or two bodies. Such coordination is also essential in different types of sport. These poses require body coordination in the context of a given exercise, they require the choice of people with similar body parameters, joint action requires cooperation and taking responsibility for the other person. Yoga from individual practice becomes a collective practice. Exercises in a direct contact with the other person activate also comparison with others. We have here an empathy read by the interlocutor and used in filmed exercises, as well as empathy connected with feelings of own body of the interlocutor based on pictures shown in the movie:

- What do you think about this film?

- Well, doing a given pose in pairs requires that one person looks after the other. It was shown here that this girl on the right hurried the pose and exiting it. I think that it is not completely good that when somebody does... we do some asana in pairs, then it should be done fluently and all the time feel what the other person is doing. Because when so, one of them had her legs straight and the other one had them bent, this one went up faster than the other one, so I really think that the aim was not achieved. And I think that people that are in pairs should also be well adjusted when it comes to height and weight. Well, it is vital because then the pressure changes and it is important.

- What do you feel while doing such an exercise?

- Well, not only do I have to keep my balance but I also pay attention to and balance between my own body and the body I put pressure on, right. So I have to feel the moment so not too hard not too soft because we go back or forward. In general, you can fall down and you need to pay attention. So, here I am responsible for the other person.

- What are the consequences for the body?

- The usual. We train, we check ourselves. But, I think that here maybe important is this that I have to remember the other person. So I learn that I am not the only one here. If I want to do something correctly, then I also have to look at the other person. So, some cooperation, how far I am capable cooperating with somebody and whether I get upset because somebody, according to me, did something wrong, or better. 
- And when it comes to such experiencing of own body... do you think that the exercises that you do on your own or the exercises in pairs, which ones lead better to that you start realizing? Do you achieve the awareness of your own body?

- Well, I think that here, I simply bring up different aspects. When I practice alone... I know myself where I have the boundaries and I try to overcome them. And if I train in a pair, then I am at the same time aware how well is the other person able to do a given pose and automatically I compare myself to this person. Whether I can do the same as this person or more or less and here I am able to compare myself to somebody. (movie-elicited interview with practitioner for a few years [auto-definition - medium advanced], Transition to Standing Pose in Partner Yoga)

Other practitioners also underline the influence of exercises in pairs on the stronger motivation to the exercises, what is more, it is easier to do exercises in pairs:

- So, is it easier to do some exercises in pairs?

- Easier, definitely easier is to do most of the things in pairs. And when the man has such feeling that it is so easy, then when the teacher tells you that if you practice hard, then it will be so easy without the partner, then ... this is motivating.

- What are the consequences for the body and the mind when you do asanas in pairs?

- It is something different than when you practice on your own because here it is about that... somebody is watching you and that is why you try and try to show from your best side. But, it is nice because s/he will always tell you what you are doing wrong, correct, this is so nice when there is a person that does so. (movie-elicited interview with practitioner for 5 years, Parsvottanasana Pose in Partner Yoga)

Doing the exercises in pairs we pay attention to the partner more than to the feelings of the body. Very important is also the trust in your partner that is built on the basis of corporal empathy:

- What are the consequences for the body and the mind of doing this asana?

- It is such a simple asana, this or the transfer from standing up to sitting, from standing asanas to these sitting ones or the other way from sitting to standing. This is, to be honest, a transfer and ... you do not pay more attention to what she is doing. You pay more attention to the interaction with this other person, I have such a feeling that when we actually glue each other back to back, then we manage to lift.

- So, trust is important, that you said, and that we do something together, such cooperation?

- Yes, cooperation in some sense, however, during the class you sometimes do this with completely strange people, but then you catch the trust, for example, in five minutes, and you know that it is cool. (movie-elicited interview with practitioner for 5 years, Transition to Standing Pose in Partner Yoga) 


\subsection{The role of the teacher and the correctness of asana performance}

The transfer of knowledge by experts is also done in a direct contact face to face with the practitioner. Doing the position correctly is possible thanks to the teacher, it is emphasized by many researched:

- When can you do this exercise completely correct?

- When can it be done completely correct? To be honest, under the watchful eye of a good teacher or with a great ability to use these audio-visual materials I also reckon, somebody can... you can already do all of these poses correctly. Even a man using three bolsters under the back has their own capabilities of doing it correctly. It is not so that only when we add the bolsters... you also need to pay attention to the back being close to the floor, to the mat and... the positioning of the hands and legs it is important. It is not such a natural pose with these legs. (movie-elicited interview with practitioner for 7 years, Supta Virasana)

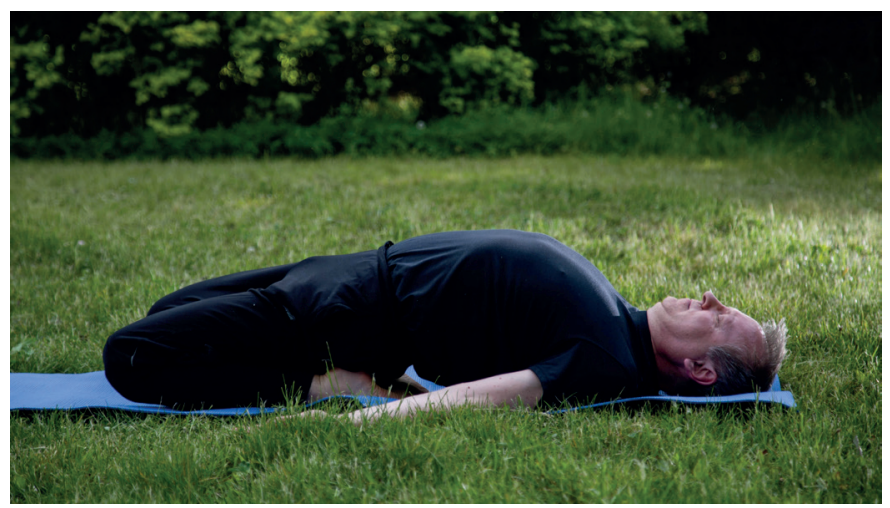

Fig. 15. Supta virasana (photo by Kamil Głowacki)

To transfer the knowledge about performing given asanas, some researched wanted to show how they do it, then their transfer about doing given poses was full and completed the verbal transfer.

Correctness of performing some asanas depends then, as it has already been mentioned, on the experience of the practitioner, his physical capabilities, but also it is connected with the role of the teacher giving instructions and correcting the practicing also by touch correction of poses:

- What do you feel while performing this asana (utkatanasana)?

- In the beginning, when you start doing it, you fall down and you are not able to keep the balance because this asana is difficult. It is difficult, it engages ankles, the ankles hurt, knees hurt, you need to hold, the back rolls at the same time, that is 
why the teacher is essential to make you straight. The arms hurt because they are stretched upwards, keeping your arms up is sometimes more difficult than doing anything else. This is not my favorite asana; I do not like it too much. It resembles such "chair" done during P.E. but only without the wall behind us.

- So, for a correct performance of this asana you need a teacher?

- Yes, if it is to be correct, yes. There are some asanas that simply seem simple, but you try to cut short so it would be simpler and then either the arms fall down or the shoulders fall down or the hands go not as they're supposed to go in a straight line but fall down, yes. And here is essential that someone who would stand on the side and say "aa" straight up, yes. I mean, when you learn with the teacher, then later at home you can also do it on your own, however, this one is such that for the beginners a teacher is needed. (movie-elicited interview with practitioner for approximately 5 years, Utkatanasana)

The teacher is needed not only for the correct performance of a given asana. The teacher also delivers the language with which we name the asanas, we feel them, and also preserve the instruction in the memory that concerns doing a given pose or breathing. The author has noticed that one of the interlocutors described in details the breath with the usage of the same words as their instructor that the researcher knew from yoga classes at school where he attended. The statement "natural breath as it happens itself" was a very common instruction of the teacher while doing supta virasana, siavasana, and other poses:

- What does a breath look like here?

- Natural, I mean, me, for example, I have no problems with this asana and here the breath is pleasant, natural, just as it normally comes out. (movie-elicited interview with practitioner for 8 years, Supta Virasana)

\subsection{Conclusions}

The feelings in yoga practice are extremely individualized. The same position can cause with one person pain and with another pleasure and happiness. Feelings are dependent on the length of the yoga practice, systematicity of the practice, body possibilities, and teacher's instructions. In general, the longer the practice is, the higher is the awareness of the body. It is also connected with a greater influence of the teacher on the feelings while doing asanas that delivers specific language to name these feelings. The types of feelings in yoga practice are: pain, discomfort, pleasure, happiness (childlike happiness), calming, peace, and even irritation. 
Sometimes, to the feelings in yoga practice some include, as it has been mentioned, pain and/or discomfort. It is felt in many poses in the body. The practitioners talk about overcoming the pain, they minimize it while telling about the corporal feelings from yoga practice. The pleasure appears in the moment of correct performance of the asana, of course, depending on own possibilities of the practitioner. Overcoming own weaknesses and pain in a given asana can be perceived as pleasant. During and after doing asanas there can also be happiness appearing, and sometimes even something that the practitioners called "childlike happiness."

The research technique "movie-elicited interview" allows better research of some phenomena than participant observation, technique of unstructured interview, narrative interview, sociological photography, or video recording of natural actions. Movie-elicited interview activates memories about the feelings of the body. Body empathy appears then, which is a possibility of a detailed technical description of the movements and feelings of the body and emotional feelings based on seen pictures. What we are not able to get from the narrative and unstructured interviews because of the reliance upon only generalized memory is made concrete by showing the pictures of a done asana in the sequence of moves. Showing the sequence of moves somewhat activates in the memory the complete positions of the body and feelings connected with them. Participant observations, however, cause such a huge engagement of the researcher in the practicing of the exercises that it is difficult to bring up in yourself distance to the object of observation that your own body is. Only after connecting these techniques, especially concentration on the data obtained by "movie-elicited interviews," allows us a fuller showing of experiencing the body. A full transfer about experiencing the body is, however, not possible. It is clearly said by the people researched with the usage of the above-mentioned research techniques. There exist serious difficulties with a verbal transfer of information about the body feelings. Intersubjective elements of communication (verbal language) allow some transfer of feelings. However, it is derivative of acquiring the language and socialization of yoga practice. The knowledge about feelings of the body is included in the body, and that is why only engagement of the body or pictures of its movement can activate it and allow, to some extent, verbal transfers to the partner of interaction. Words are not often "the quality" of a given action, assurance of complete understanding of a given situation (Dewey 1931). It often happens to perform and present asanas directly to the researcher. It is somewhat a transfer of knowledge by "real show" (by an example). Intersubjectivity could 
be created with no words, even by silence, the connection of people could be created by "embodied involvement in the same event". (Pagis 2010b: 324)

My research shows that the transfer of the non-verbal knowledge and feelings of the body and outer-verbal embodiment of this knowledge connected with the body is possible with the usage of many resources, including visual, verbal, and transfers by example and showings of specific movements of the body. The technique of movie-elicited interviews seems very helpful in the research.

It seems that this technique can have a special usage in the research strategy called "the visual grounded theory" where visual materials are used for the research of visual social processes (Konecki $2009 ; 2011 \mathrm{a})$. The above example of the research on the yoga practice shows that we can research, for example, "visualization of yoga practice" using not only the analysis of the photography or video recordings but also movie-elicited interviews. Linguistic data was generated by the usage of this technique allowing the showing of "some layer of data" existing in the statements based on the presenting of the body moves in the film. It is a layer of data concerning the remembering of the bodily feelings expressed in language and activated only in a situation of replication of these moves shown in the movie. The multitude of the data can be generated this way during the movie-elicited interview, which is so important in the methodology of grounded theory. 


\section{CHAPTER 6}

\section{Emotions and yoga practicing. Working on emotions and achieving "emotional culture" without emotions}

\subsection{Introduction}

Yoga in the modern version, directed mainly to the Western receiver, for example, in the shape of so-called hatha-yoga, is directed to achieve therapeutic effects. Its modern propagators adjusted it to the needs of the man feeling strong civilizational stress caused by the pressure to venture so-called career dangers connected with nutrition, environment pollution, noise, pressure on consumption, and also with the institutional secularization of the modern society, etc. Thanks to it, in yoga schools, "work on emotions" that is done by specific physical practices (with the usage of the body) in order to gain emotional stability, elimination of negative emotions, and obtaining mental peace can take place. The work on emotions seems to be an internalized ritual of "modern religion" that most often is not seen for a side viewer and even for working on emotions. Work on the mental and physical health is a "boundary place" where the aspirations of the profanum sphere (practical goals of the individual) meet with the aspirations of the sacrum sphere (spiritual goals) of a given individual whose aims are the need to overcome own possibilities and existential fitness to become somebody else, so the old body would die and the new is born with a freshened psyche and in general without emotions. The work on emotions in hatha-yoga has thus an initial character if there is a visible change in the individual confirmed by its new interpretations of own emotions and own feeling.

I would like to show in the chapter what work on emotions in the social world of yoga practice is. The second goal is an attempt to interpret this phenomena in the light of the processes undergoing in the modern Western societies.

That yoga helps to shape the emotional stability is written by hatha-yoga's guru B. K. S. Iyengar (2005b). According to Iyengar, with the use of work on the body, you can get rid of the stress. Iyengar's attitude to yoga has a therapeutic character; it concerns both the physical and psychic sphere. According to Mark Singleton (2010a), this therapeu- 
tic direction of yoga development was only started at the turn of the $19^{\text {th }}$ and $20^{\text {th }}$ century when, under the influence of Western gymnastics and accompanying ideology to improve the health and frame of mind, Indian yoga developed as we know it in the West. In the development known to us, yoga has also used inspirations from ancient India, as well as achievements of physical culture and academic medicine of the West. The pioneers of modern hatha-yoga, like Swami Kuvalayananda (1883-1966), used achievements of modern science to measure the physiological effects coming from doing asanas (Singleton 2010a: 115-116). Similarly, another precursor of modern yoga, Sri Yogendra (1897-1989), accented the rational, utilitarian, and academic dimensions of yoga and its therapeutic effects. Yoga, according to him, has to develop the body, mind, and other psychological potentials harmoniously, which in the end leads to physical well-being, psychic harmony, moral up-lifting, and spiritual awareness (ibid.: 118). His vision of yoga is also a preventive medical practice.

The social world of yoga practice has its own culture of emotions and emotional ideologies (Hochschild 1983). The culture of emotions of this world is, in general, connected with pointing towards so-called "emotional disturbances." Emotional ideology in general is connected not with feeling the emotions and/or not bonding to them if they are felt. The rules of feeling the emotions usually do not point to the specific emotions, but generally it is about liquidating emotional disturbances so that the possibly felt emotions have a "neutral character," that is, so the subject watched them and did not attach to them. The social world of yoga practice delivers also a specific vocabulary of emotions (Shott 1979). This vocabulary is built already on the level of emotional disturbances, but also later by the participants themselves when they describe what they feel during yoga practice. Thus, the situation of practice (work on emotions) is described with specific rules of feeling. What we feel, according to practicioners, during practice is: a dissolving of emotions, harmonious feelings, liquidation of tensions, and flow of positive emotions in the form of "prana" (energy). Above that we learn to "control" emotions, patience, emotional empathy; and, all of this has a positive influence on the relations with others. These emotional feelings were described by the practitioners in the interviews and also felt by the author of this research and this monograph. Personal experiences of the author, taken from the participation in the social world of yoga, surely had influence on the interpretation of the results of the research and also individual (connected with the place of the author's practice) and wider context of social research, thus, observation of the social need for hatha-yoga 
and other such practices. Emotions felt by the author of this work, by the work on the body, were definitely an additional source of knowledge (compare Wyka 1993: part II, paragraph 1.9).

According to Iyengar, the stress in our culture is connected with the so-called "rat race." "The 'rat race' has created much unnecessary tension both within us and around us... Emotional stresses imprint themselves on the physical, organic, and neurological bodies just as music is imprinted on a compact-disc. Even animals fall sick and die from emotional stress" (2005b: 77-78). According to Iyengar, "The main causes of negative stress are anger, fear, speed, greed, unhealthy ambition, and competition which produce deleterious effect on the body and mind" (ibid.: 79). Yoga practice influences the autonomous nervous system and can be used in curing epilepsy, depression, and after-shock stress disorder. ${ }^{1}$

At the basis of negative emotions is the care for own ego. Ego wants immortality and wants to provide it with earthly goods and consuming life style: "It is egoic ME that does not want to die. This impersonation of soul by ego is at the base of all human woes and this is the root of avidya (ignorance or nescience)" (ibid.: 193). We last in fear of perishability that evokes negative emotions. Work on ego is the reason for pride that, according to Thomas Scheff (1990), is a positive social emotion because it strengthens the self-respect and you can build a harmonious social relationship on that. However, from the point of view of yoga philosophy, it can actually be the other way around, attaching ourselves to the feelings of pride, we attach to ego which unsatisfied with praises, prizes both outer, as well as inner suffers. Fear appears that the ego can be unsatisfied also in the future. Orientation for the future is a feature of both the Western culture and the "politics of individual ego" that is well written into this culture. It is a completely different attitude to the issue of ego that it is in the Eastern tradition where yoga comes from. We want to defend ego, although we should discard it in yoga and every action should be without desire:

Nishkama karma (Nish - without + Kama - desire: karma action) is an action performed without a wish or desire for the "fruits," or results, of such action. The ultimate yogic ideal is to perform all actions without personal gain, and to offer instead the fruits of all actions to God. Performing actions with the mind directed towards results increases ego and keeps us bonded to the idea of "I" and "mine," while offering the fruits of actions to God leads towards surrender to the divine will and the liberation from the idea of a separate self. (Pattabhi 2010: 3)

${ }^{1}$ See: http://www.ncbi.nlm.nih.gov/pubmed/22365651 (accessed: 8.03.2012). 
In general, modern times give us many reasons for living in fear and anxiety. We live in the concern about the future to save what we have gained, not accepting the style of life as it is here and now. Adjusting oneself to permanent life changes is possible with control of emotional disturbance that we are liable to. One of Patanjali's precursors also mentions the emotional disturbances (see: Iyengar 2002a: 8, 88, especially sutra Kaivalya pada [225-257]).

There are six disturbances of emotional sphere: "Thanks to yoga we are able to weaken six obstacles of emotional nature that cause us such great torment. These are lust, pride and obsession, anger, hatred, and greed. According to Western psychology, these are negative emotions, and Christianity considers them as main sins" (Iyengar 2005b: 82). Iyengar continues to state that a great meaning in building and acting of emotions has the ego: "To understand the emotions, we have to recognize the role the ego plays in them" (ibid.: 83). Negative emotions can be, however, used in positive aims: "Similarly, our emotions and intellect must be developed for divine purposes" (ibid.). "Emotions lie in the physical sense in the organs of the physiological body - at the level of the pranayama kosa" (ibid.: 840). They, however, move also to the sphere of memory, they become a mental phenomenon and acquire permanent features. We often objectify the emotions, even though feeling them has a processual character (ibid.: 85).

In the Eastern psychology, emotions themselves are not distinguished. They appear in connection to the actions and their moral assessment. Only in this context can we talk about the obstacles of emotional nature. First disturbance of emotional sphere is "lust." It is strongly connected with ego and the need of power (ibid.: 87).

Another emotional obstacle is "pride and obsession." We want to persuade others to do and think as we do and think. Ego also appears here, because of presence of pride. We are the slaves of our ego here (ibid.: 87-88).

Anger also comes from protecting the ego. Attachment to ego creates negative emotions: "This is the anger that comes from the ego. Another car cuts us off in the street and we feel offended. 'He cuts me off,' we tell ourselves. He did this to me" (ibid.: 88).

Negative emotions written in our memory is hatred, it is the last of the emotional disturbances mentioned by Iyengar. It can activate not only under the influence of a direct outer stimulus. If it is nourished by the subject, it will also show in the moment that she/he decides. It has destructive influence on people's actions, it can be observed there where is a lack of tolerance, violence, and war (ibid.: 90). 
Greed is again connected with the craving for power, it is its derivative, and having power we want more and more satisfaction of our needs. But, we are never satisfied and never content. Greed comes from our fears. Yoga helps us to get rid of fears (ibid.: 92-93).

Yoga allows thus to discard the fears of the modern world. The workers in the West feel, nowadays, pathological fear because of the threat of losing their job or position. The feeling of a lack of security and increased individual responsibility in the world of competition results with the feeling of fear and danger.

27 million people in Europe, more than one-fourth of the population, suffers from 12 of the most popular mental illnesses, one-third have anxiety attacks and panic attacks. The European Brain Council estimates annual costs of curing mental and psychosomatic illnesses in European countries to around €386 billion. In Germany, according to the statistics of ill people (DAK), the percentage of "absences at work caused by anxiety disorders" increased from the year 2000 to the year 2005 by $27 \%$; in the case of depression, during the same time, the increase was $42 \%$. In total, almost $10 \%$ of doctors' notes in Germany are connected with mental illnesses... Often the patients with anxiety disorders are egocentric. People suffering from social phobias are afraid of being judged by others because it is their judgment that is the way to social appreciation. That is why they work hard on themselves, setting in front of themselves high demands; they are perfectionists and very hard working people. They do not know what complaining is; they dig themselves into work. No wonder they have their employees sympathy (Schüle 2007). ${ }^{2}$

To get rid of these disturbances one needs to reach inner peace using prudence (vivecana): "But, in order to be victorious over six causes of delusion or emotional disturbances, one has to use the six spokes of the wheel of peace. These are: discrimination and reasoning, practice and detachment, and faith and courage" (Iyengar 2005b: 93).

The first advice that Patanjali gives us about these disturbances I will translate very loosely: "If you are happy, pleasant, and unselfish in your behavior towards others, obstacles will shrink. If you are miserly negative and judgmental in your mind, the obstacles will grow." More precisely, what Patanjali said is this. In order to achieve a serene consciousness, we have to be willing to change our behavior and approach to the external world. This is for our good. Certain treatments known, as the Healthy and Healing Qualities of Consciousness, cultivate the mind and smooth the yogic path (Iyengar 2005b: 94).

What is recommended by the masters is most importantly the practice: the practice of asanas and pranayama, practice of a friendly attitude towards the happy ones, the practice of compassion towards the

${ }^{2}$ See also: http://www.zeit.de/2007/17/Dossier-Angst (accessed: 9.02.2014). 
sad ones and nourishing the happiness towards the ones that are full of virtues and goodness, neutrality and indifference towards those full of sin. What is necessary in the authentic practice of yoga that Iyengar talks about is the practice of "withdrawal" (pratyahara). The beginning to this is given by pranayama where with the control of breath, the consciousness, and energy are directed inwards. Pratyahara allows the withdrawal of the senses and also the mind from assessing the outer world. It gives possibility to see the reality in a fresh and not be infected with our concepts, ways, habits, and acquired criteria of assessment. We can then concentrate, separate from our desires, we are "indifferent" to the world in the sense of its reception here and now, we do not judge it. Prana is an energy that we need to live and be healthy. Only its unblocking allows healthy living. Negative emotions do not allow this vital power of the world to circle freely.

Emotional stability is thus not only a derivative of clear corporal practice, performance of asanas, it is connected with the work on own attitude towards others. The work on the attitudes is hence a work on social relations where positive attitudes give possibilities for positive emotions to appear, for example, happiness and peace. Although it is widely spoken about performing asanas as a technique of achieving emotional peace, the work on the body itself is, however, only a shortcut that can be easily concluded from the above remarks of Iyengar. It is also necessary to underline the strong relationship of yoga philosophy with the pressure on the therapeutic effects of its practice both in the physical (healthy body) and mental (calm mind) aspect. Yoga, apart from that it shows that the life path, also cures. Curing from negative emotions, it also cures the body and vice versa. Maybe this is the main reason of its popularity in the Western world because for many it is not only the prevention but also the medicine. We see it as a process of medicalization of yoga practice.

A different part of the quoted book (Iyengar 2005b: 267-270), Asanas for Emotional Stability, shows in details what can be done with the usage of the work on the body to achieve emotional stability. The same asanas are shown that help to cope with specific emotions, some of them quite the mind and cool the brain (adho mukha svanasana, uttanasana, sirsasana), others stimulate the brain (upavishta-konasana, baddha-konasana) to positive thinking, others bring peace (supta-virasana), allow to experience inner silence (viloma-pranayama). Some asanas are advisable for people suffering from migraines (ibid.: 267270). Performing these asanas is hence a work on emotions.

Such a way of thinking about yoga and the language of description presented by B. K. S. Iyengar is favorably accepted by the Western cul- 
ture. The search for emancipation and relaxation is vital in the culture of pursuit and eternal haste and strong stress. Everyday life builds conditions for the stress and other ailments connected with psychic tension to appear. "Health justifications" for practicing yoga are very convergent with the expectations of people looking for mental relaxation in physical exercises in the Western culture.

\section{$* * *$}

We need to make here some methodological remarks. The notions of emotions and work on emotions as the categories of analysis were introduced in my research rather late. They are not dominant in the complete analysis and are not leading notions. These are outer notions, introduced on some outer, in relation to the research on yoga practice, theoretical reflection, that helped me to recode the data that was coded according to other patterns and different outlines of categories (becoming the practitioner, constructing the identity of the practitioner, the role of the teacher, the corporality in the process of becoming the practitioner, spiritual aspects of yoga, etc.). All of this shows that the leading notions direct the analysis of empirical data in a given way. It does not mean that the outer notions introduced to the analysis falsify this analysis and the data in any way. The same data can give clues for other directions of analysis, although the same area will be always researched. The data is obtained from the same respondents and will refer to the same actions, but only seen from a different angle. The reality is not created here; its theoretical view is constructed here, which is an interpretation. Interpretations are the derivatives of the concepts. The concept of emotions, and especially the work on emotions, introduced during analysis of the data can be such sensitizing concepts raising the awareness of a specific direction of analysis, or they can even be introduced earlier if, for example, they appear earlier in the process of the research and direct the collection of the data and statements towards different types of emotions or the work on emotions.

\subsection{Working on emotions - explanations based on practitioners' statements}

Work on emotions that we deal with here concerns the phenomenon of the change of the meaning of emotions that appeared on the surface of our consciousness. Emotions that we, in our everyday life, 
pay attention to are supposed to be transferred into emotions not important or less significant for our life and living life. We gain distance from them. Through work on the body we are able to get deeper in reality and see the passing of emotions. Work on the body becomes here the work on emotions. This work is done by an individual without the concentration on the content of the consciousness and the experienced strong emotions in everyday life. This work is done individually without paying attention to the social contexts of appearing of the emotions that has a meaning in the emotional work that Arlie Hochschild (1983) writes about. It is not about disconnecting "private I" from the "public I" but about defining the meaning of any "I." It is also not about depersonalization of social situations in which we experience negative emotions when we are, for example, criticized (compare Hochschild 1983: 38). It is more about redefining the actions and emotions accompanying them, but with the addition of distance to own ego. These redefinitions are rather connected with becoming aware of the "constructive character of I" and the fleetingness of the feeling of emotions and the ego itself. In general, such types of changes happen on a deep level, unconscious, during a longer period of practice. Having an effect on the body that is closely connected with the mind, we also affect the consciousness and the need for a new defining of emotions, ego, and everyday life.

Below I present the issue connected with the work on emotions and feelings connected with this work from the perspective of the researched. Yoga practitioners recognize the relation of emotions with the body, its condition, health, etc. Emotions, according to the feelings and reports of the researched, locate in specific parts of the body. If we recognize the body parts and emotions connected with it through the practice of specific asanas, we can "dissolve" them. The observation of given movements, even held in a given asana, allows recognizing the emotions and tensions in the body, according to the researched. Of course, the fact of observation is here compared with meditation where one observes the flowing thoughts not interfering in them nor stopping them:

This is, as I have said, with that exercise that there were there some emotions; in general, it's based on my experience, for some people, it can seem stupid, but that somewhere there on the energetic level all these emotions are somewhere in the body and they find places in different body parts, right. And this is what you feel during meditation and also during exercises, where... the same the positioning of the body has an influence on emotions, I don't know, on the basis of some simple experiments I sit normally in my room, to observe how you sit, how it influences you, how you feel then. So, in some sense you can talk about emotions, and just as I said 
with this exercise with it I felt some short circuit in my chest, even though the chest exercises in the last moment, that in that stage I was yet unable to engage in the exercise. So somewhere there, this specific pose... well, somewhere it awakened this place where the tension was. Also when you stay in this pose for longer, when some unpleasant emotion appears in you, or some blockage or muscle tension, and when you stay in this pose and don't act like they tell you in the aerobics that " 1,2 , 3,4 ," that you repeat this movement and you deepen it at most, you dissolve this move. And this is again an analogy to what happens in meditation, if you wait for it to pass because it will pass, right. And if you force yourself to do something, stretch, then your body will start defending, starts blocking. The only thing you can do is to observe and wait. It teaches observation. Yoga, above all, teaches observation.

If you put a pose, then first you feel that something is happening to the body, right. Then you become aware that you feel and you start observing. You start observing so you start being aware of what's going on, for example, if you change that move, you adjust it a bit, such minimal things that somebody from outside is not able to perceive, and you start observing how these small changes influence the whole move, the whole pose, and how it influences you, somewhere you surely start intuitively feeling how it all should look, right. That it should be symmetric, that it should be with a straight spine, that it should straighten the whole body, not to forget about the feet, hands, and such details. (practitioner for 5 years)

You can also come with emotions with the usage of breathing exercises that accompany yoga, like pranayama. Scientific experiments show that yogic breathing exercises reduce stress, anxiety, and depression. ${ }^{3}$ We control the breath in pranayama, however, in meditation itself we observe the breath, where the stress and negative emotions also show. Holding the breath in tension is thus valid because together with its end emotions pass:

Well, rather before... to this yoga that I used to go to then this mediation is really short, it is after... after breathing exercises and what else is here to say, breathing exercises work quieting, so it is definitely easier to meditate after them rather than start meditating when you just walked in from a noisy town where the thoughts are scattered, right. It is easier after the breathing exercises than in such a situation. Hmm... me, for example, while meditating, remember the first stage was concentration, it was not meditation but concentration and it was concentrating on breathing, on the sensations that are there somewhere in the lungs, on a wider area somewhere there in the nasal cavity and then in the exit of the nostrils and... and this also is, for example, because with pranayama you breath in such a way that you control your breath and, for example, while meditating, there is no such thing, you only observe, you do not try to influence it. And if in this moment it appears that you have some suppressed emotions, stresses that means... it starts appearing in the breath. But, you cannot block it, you just only wait for it to pass. (practitioner for 5 years)

\footnotetext{
${ }^{3}$ See: http://www.ncbi.nlm.nih.gov/pubmed/22365651 (accessed: 8.03.2012).
} 
Work on the body leads to harmonious feelings; tensions are liquidated. The body puts up some resistance but after overcoming it, there is satisfaction from overcoming restrictions. Body resistance also states that stress accumulates in specific parts of the body. After overcoming the resistance you feel satisfaction, positive emotions:

Well, right, the same when I feel that somewhere during these exercises next parts of muscles let go, then in the same way you feel freedom, this breath lets go, relaxes, and this psychic relief is big. For example, a big difference is when at the beginning you start exercising and, to be honest, the body resists, and you do it forcefully... I mean, you should not be doing anything forcefully, but you feel this resistance and that it is more of a fight. And the moment you reach this first stage, when you can do the exercises freely, then... I don't know, I, for example, feel such harmony. To be honest, I feel that when I do some exercises, do some pose, hold it, I can deepen it, I feel my muscles, I feel them let go, then I start feeling harmony with myself, peace, pleasure... it also gives satisfaction. True, I feel satisfaction from it, that you can do some things, that you overcome yourself, that you cross your boundaries. It means that the details are more important and to do these exercises correctly rather than to do it the hardest, and when you feel that the body puts up resistance, then somewhere there is some amount of stress being released, right. (practitioner for 5 years)

The body resistance is correlated with the fears that we have in ourselves. Performing some asanas allows overcoming these fears because before doing and while doing these asanas we experience these fears, however, performing the asana allows us to overcome these fears:

Consultations during brakes take place with Michał who explains many doubt. For example, concerning pain of the lower part of the spine, whether you have to exercise generally to get rid of it. One lady was afraid to do Supta Virasana because she was afraid of a great pain and that it would damage her already sick spine, as she stated. This time, however, she did Supta Virasana and as she stated "nothing happened." She was pleased because of this and she underlined the fear of doing this asana. Michał asked her "And how are you feeling?" And she said that she was OK, that she overcame the fear and nothing happened. "You see," said Michał. (participant observation and auto-ethnography, November 10, 2008)

The awareness of the body that appears during the practice of asana liquidates the tensions. In the quotation below, we have a description of how to do supta virasana (with some modifications). The author of the description had full awareness of what happens to her body, she perfectly remembers feelings that are born during exercises. Although, she is sometimes lost for words to describe the feelings, as it is seen in one of the last sentences of the below quotation. Also the time when you exercise is important. Morning exercises "melt emotions," prepare 
the practitioner for further actions during the day. Dissolving the tensions in the body leads to dissolving emotions connected with them:

\begin{abstract}
And when I feel that I relax a bit, then I start working with the chest, looking up, tilt the spine a bit, right. And then I feel I start stretching the hip more, awareness is in the leg that is bent, right, in the foot that is leaning against the wall, but also in the other leg, the one in the front that holds the balance, there is the feeling all the time that you think that, for example, that the tension between this and the bent legs left and right is on your balance depends on this tension, the positioning of hips, whether you have them parallel to the wall and whether you do not tilt them to one side or the other, you think about balancing, this position of yours is now straight, symmetric, your spine when you start leaning back, there is no fear that, for example, it is not even and it will be crooked and maybe these spaces between discs on one side will be overloaded too much, and then we lean even more, all the time in this, in this balancing, these muscles of the left hip I bend them, all the time in this, in this balancing, these muscles of this left hip start letting go and the muscles of the right leg also start working, muscles of the right thigh, the ones like for the split a bit, and then you also go down and at the same time you tilt the body back and again the front starts working, chest, all these abdominal muscles, you grab the ladder with your hands, and again this body, again this body, this leg, you feel the whole, as if there was somewhere in this leg some path of tensions, that if the right leg is the supporting one, but your left leg and your torso with your legs create an arch and you simply feel this tension in this arch, you know, such harmonization. And you also enter, deepen this bend, right. And it also works at the back, at this moment it is overloaded a bit because it is (not understood) also change, but in general, it also works relaxing, I think so. Because even thanks to, for improving the back, when you are bent like that and what, right (three not understoodable seconds) when you hold the pose and wait and dissolve all these tensions, right. And what, later there is this version of this exercise that you let go and you lean forward, dang it I don't know it word for word.
\end{abstract}

I also sometimes feel stress when it leaves me in the morning then you are somewhere there inside, such that the muscles shrink, I am so, oh, not calm, so this is also proof in my life that at least my body works so, that in the morning there are these tensions. And, in general, it's good for me to exercise in the morning. (practitioner for 5 years)

Liquidation of tensions is one side of coping of the practitioners with the emotions. In general, this is a fight with stress that above-mentioned tensions are indicators. The other side is the breath control while doing the asanas; difficult exercises calm you down and give a feeling of harmony. You achieve an effect of relaxation of organism:

I, after a difficult week, went for two hours to do yoga and left very calm, relaxed, apart from that, tired, because, after all, these are difficult physical exercises, it really requires being fit, that you breathe, that you control the breath, then this organism calms down, inner harmony can be achieved thanks to that. Really, since I had contact with yoga then even my attitude to life has changed, I am really an 
irritable person, impatient, and it is yoga that calms me down. (practitioner for many years, age 24)

Apart from gaining calmness and harmony other meanings ascribed to yoga practice is control of emotions that do not allow the stress to appear:

I don't care to show off that I do yoga and I am fashionable (laughter). Besides, now, many people sign up for class, on one side this popularity and on the other side stress and fast pace of life, this all overwhelms and people begin looking for something that will allow them to be free from the everyday noise. This something is often yoga because it allows you to control emotions. (practitioner for half a year age 40)

Controlling the emotions is also learning patience by precise performance of asanas:

- For me, the precision is the most important because this yoga is not just as if but this woman is really... the way she runs the class she makes you focus so much on what you are doing and every... even the smallest detail of arranging your back on the floor is really important, if you adjust them incorrectly, then you are simply lost and this exercise will not work. So the precision here is the most important.

- And for you?

- And this is what I like because it teaches me patience because I am not patient and this yoga teaches me patience, I am hot tempered and do you imagine doing one exercise that seems so trivial to you for five minutes? No, I also thought that this is undoable, but this is why after this yoga I leave so calm. (practitioner, ethnology student)

After finishing the yoga practice the practitioners leave cleansed from their "problems," these problems usually have an emotional character or are connected with emotions.

Control of emotions is possible thanks to learning how to observe own emotional behavior and transferring it into understanding the behavior of others. During yoga practice we somehow learn emotional empathy, we remove here one of the disturbing emotions that anger could be:

Understanding your weaknesses, we understand the weaknesses of others. When someone sometimes shouts at us in the car, yes, when we drive somewhere and the other driver gets so upset then we are able to understand it, we do not shout straight on, show our fists, it's not important what happened, whether we cut somebody off, we drove too slow or too fast or... it's not important what happened but how we see it. We know that anybody can be under pressure, some experiences, it takes only a small incident for it to pour over, yes, so he would not be able to deal with it, and then it makes some trivial thing causes him to explode, right. But 
because we practice yoga, we are aware, we know about such things, and when we know, then we do not take it so personally but rather we simply know that it happens sometimes. Sometimes the emotions are too strong and have to be freed because that man would simply get sick instead of coming out, yes, not to allow it to get inside and cause a sickness. Well, in a nut shell. (practitioner for 14 years, yoga instructor, hatha-yoga school owner)

Emotional empathy is here an effect of undertaking the role of other, which is an element of social control (Shott 1979: 1324-1325), in this case, ideological indicators are the basis to show the emotional ideology of the social world of yoga practice, so as not to feel negative emotions. Thus, social control does not concern only the learning element but also emotional feelings that are the element of undertaking the role of the other (ibid.: 1330).

All of these influences of yoga on the individual, its psyche and emotions, are connected in the end with the influence of yoga on the relations with others. It can be a positive influence, but a negative one can also happen. The negativity of this influence can concern feelings of pride and advantage over others. It is one of the disturbing emotions mentioned by Iyengar, that is, "pride and obsession." You can, of course, liquidate this kind of feeling by realizing that:

- Yoga influences my whole life. And it influences interpersonal relations a lot. If I am... if I recall during my practice of yoga some specific qualities, then I emanate with positive quality. I personally cannot judge myself because we are never objective towards ourselves. However, I see with other people that train yoga, I see how they change in the outside world, but not always in a positive way.

- Not always (with disbelief)?

- Not always. There is something that could be called negative effects. That's when we start experiencing something deeper, when we start understanding some things wider. Sometimes there is something like ego appearing, like pride. It seems to us that we are better than others and sometimes it can be an obstacle in relations with others. Of course, this is a thing that needs to be treated as a challenge to be overcome. However, I personally catch myself that... that such types of problems exist.

- Like pride, yes? The feeling of superiority.

- That is the way it is. You need to be aware of this in yoga, so not to follow in this direction, that we are better, but rather to develop in oneself good qualities and improve oneself on all levels of our person, also in relation to other people. (practitioner for 14 years)

Because yoga lessens the level of aggression, it can influence prosocial attitudes of the practitioners and improvement of relations with others. Yoga is used, for example, as a treatment of resocialization in one of the Indian prisons in Madhya Pradesh: 
For every one-to-three month program of yoga lessons completed, the prisoners receive a prison reduction of 15 days.

"Yoga is a good thing to control the anger and to manage the stress," said Sanjay Mane, Madhya Pradesh state's inspector general of prisons. "The prisoners inside are under tremendous stress away from their families."

Mane estimates that more than 3,000 prisoners have taken the courses held each morning. The classes focus on breathing exercises and asanas, or yoga positions. If the prisoners take the classes seriously, it is considered good conduct and can be reviewed for a reduction of sentences. He said the prison system does not know whether learning how to do the downward dog and other yoga positions has kept inmates from landing back in prison and said they need to do a study on recidivism. Some critics suggest that yoga cannot transform a criminal into a respected member of society, but Mane thinks otherwise.

"Some people have their doubts, I do not have any doubts whatsoever," he said. "They're better off than their earlier phase and in control of themselves."

Interpretations of yoga and its influence on mental health, including emotions and morality, are concurrent with the cultural interpretations of yoga in India, interpretations that are reflected in Iyengar's works.

Some of the exercises in yoga practice add free energy in the people exercising. What is this energy? It is some inflow of strength on the level of body physiology, but also an inflow of positive emotions that at the same time of releasing let the individual to act with a greater power. In general, it is difficult to describe this energy; the interlocutor gives its Sanskrit name "prana," and this closes her statement and explanations:

There are breathing exercises that act energizing, airing - for the circulation, and so on, and there are exercises acting balancing, for example, you cover your nostrils, breathe in. You always do this exercise from the bottom to the chest so the pressure would not go up, you cover the nostrils a bit, breathe in with one nostril, breathe out with the other, then you start with the other one that is breathe in, the other one, and the other way around, and then you do it in both - breathe in with left then right, then left, then right, and this is balancing. Such breathing exercises are connected with the attitude on the energetic level that this breath is; this prana, it is said, that it is, well, I don't know how to say this. (practitioner for 5 years)

\subsection{Conclusions}

The practitioners use a specific vocabulary naming what they do in the work on emotions and what they feel. The social world of yoga practice has thus its own culture of emotions (there is a specific vocabu-

${ }^{4}$ See: http://abcnews.go.com/Health/International/prison-yoga-inmates/story?id= 9691136 (accessed: 2.08.2011). 
lary of motives [Mills 1940: 904-913] and suggestion that "emotional disturbances" need to be liquidated) and emotional ideology (suggestion not to feel emotions, and if they are, then not to pay attention to them). It can be said that emotions are not only physiological reactions of an individual but are the result of learning the interpretations and social influence (Shott 1979: 1323).

This work on emotions is about so-called "dissolving of emotions," often by observing them that allows us to gain distance from them.

"Dissolving of emotions" is connected with liquidating tensions in the body by body awareness and placing emotions in it (tensions), and body moves (as freeing the emotions) which leads to achieving peace and harmony. The second effect of the work on emotions is, according to the researched, "controlling emotions" by, among other things, learning patience or endurance through inconvenience and pain while practicing asanas. Work on emotions is also learning emotional empathy.

You do not fight with emotions in yoga; you do not damage them. Observing emotions allows seeing their changeability, fading away, and not substantialness. Thanks to this the practitioners do not attach to them at all or pay to them less attention in their everyday life. Emotions do not control the practitioners, one can say that the practitioners in some sense "control" emotions, although it is not control from the point of strength and superiority of the mind on the emotional sphere. It is rather awareness of emotions (e.g., awareness of anger, annoyance, or pride), and if it is even possible to talk about control here, than it is "control through awareness." Becoming aware of the perishability of emotions causes the individual to be able to distance from them. The individual then moves from the direct submersion in a specific emotion, for example, anger, to a different state of awareness. It is somewhat a change of the universe of a discourse from a direct feeling discourse, often only on the level of the body, emotions to the universe where the emotion becomes observed in the whole context of a previous situation, named, and its fate is followed by the individual (compare Dewey 1931: 5). Anger exists in everyday life and can be perceived by reference to the whole situation in which it appears and actions appearing in it. However, the individual may not perceive it by itself in a given moment when it yields to it. Perceiving it as an emotion transfers us to a different universe of discourse where the accuracy of our deduction depends on a direct perception of the "full quality" of the previous situation (ibid.). The dominant leitmotif in this new universe is connected with acquiring distance, being the observ- 
er. The individual somehow changes the frame for the interpretation of the phenomenon (Goffman 1974). In the new universe of discourse, the emotions acquire a processual character. The individual cannot identify with the emotion and make it real. This interpretational whole allows for the understanding of emotions from the previous state as fading. Thanks to yoga, emotions gain place in the new universe of the discourse where they are perceived directly in a new light, not as real existence. If the new frame, hatha-yoga practice (fading away of emotions, their unreality, discarding of troublesome friendship, distance to ego, etc.), is imposed on the frame of everyday life, then the individual frees itself from emotions in some way.

In case of practitioners, work on emotions is also freeing from stress. In Great Britain, in some research about hatha-yoga, $80 \%$ of those regularly practicing continue the practice to liquidate the stress (Hasselle-Newcombe 2005: 311). But, 85\% of practitioners feel that the practice gave meaning to their lives. Hence, the researchers here perceive some element of spirituality. This is the motive that was so often very important when making decisions about starting yoga practice.

We make the hypotheses:

1. Removing stress and negative emotions is possible through work on emotions.

2. Work on emotions, on the other hand, is done by the work on the body and mind.

Maybe this is why yoga practice becomes so popular in the Western culture where the popularity of different kinds of physical and psychological occupations freeing people from stress is extremely high. Removing the stress and negative emotions became a business for many companies and professions (e.g., hypnotherapist, workshops on relaxation techniques, teaching different Eastern techniques, teaching and workshops of so-called "emotional intelligence," managing stress, positive thinking, anti-stress training, etc.) (see: Bennett 2002). There is something appearing that we can call emotional business. Also, the master of hatha-yoga, B. K. S. Iyengar, writes about freeing from stress and negative emotions in the modern world adjusting his philosophy of yoga to the needs of the Western people. A business chance for experts from the East is a fast pace of life and stress felt by the people from the West. Among the analyzed 172 websites of yoga schools in Poland, for 140 schools (81.4\%) psychic benefits connected with improving the psyche's well being are important in autopresentative strategy, and for $73.3 \%$ important are benefits of the practitioners connected with emotional emancipation.

What does hatha-yoga bring in to our world? Hatha-yoga creates an emotional culture without emotions. Dissolved emotions 
or those observed and made aware lose their power to interact. Social life without emotions, especially disturbances, is much happier. The paradox of this statement is on such "work on emotions" that does not, in general, shape emotions but removes them (both positive sign and negative sign) from the field of one's actions, cleaning its mind and leading it to a state of complete peace and balance.

Work on emotions that has always been a part of Christian religion taught and confirmed by institutions (commandments of love of the neighbor, forgiveness, discarding of hatred, peaceful attitudes are still taught by Christian churches) become, in yoga practice, a form of an invisible religion (Luckmann 1967) connected with transgressing own emotional limitations and achieving by the individual peace and harmony with the world in its inner experience. The individual working on the body overcomes its biological conditionings. This is thanks to work on emotions and at the same time on the body that has a relatively intersubjective character (is observed by others and understood by emotional empathy and descriptions similarly language-like); work on emotions finally becomes subjectivized to certain forms of spirituality that allow the individual to transcend own biological limitations connected with submersion in the commonsense world that brings it constant emotional problems and stress. The individual overcomes the limitations connected with attachment to ego. Work on emotions, as a form of spiritual and active (also corporal) reflection, becomes a domain of private spiritualities of the individual in the modern world, although explainable by description of actions leading to relaxation and freeing from negative emotions and stress. This spirituality shows also a ritual level of yoga because yoga practice is conventionalized and the way of performing given asanas is strictly specified, similarly as asanas opening and closing the sessions and sequences of performing the asanas. Taking into consideration the phenomenon of emotional business, you can say that the spirituality, just like religions, undergoes the process of commercialization.

The pluralistic situation is, above all, a market situation. In it the religious institutions become marketing agencies and the religious traditions become consumer commodities. And at any rate a good deal of religious activity in this situation comes to be dominated by the logic of market economics (Berger 1967: 138).

The religion can be sold much easier if it is shown as suitable for private life rather than when it is advertised as allowing specific usages in large public institutions (Berger 1967: 147). Thus, including economical exchange in the world of religion and para-religion does not cross out the search for spirituality done by the work on emotions. Spirituality becomes the product undergoing sale and client control. 



\section{CHAPTER 7 \\ Teacher and guru in hatha-yoga practice}

\subsection{Introduction}

A teacher who has a great authority confirmed in a line of inheriting the practice from grand masters, being a morally significant other, is called a guru. The teacher allows overcoming the obstacles that appear on the path of the pupil. It is an extremely important task for a guru:

The syllable gu means darkness, $r u$ light. Guru disperses the darkness and brings enlightenment. The notion of guru is full of deep sense. He is not a usual guide. He is a spiritual teacher that teaches the way of life and not only how to earn for this life. He passes the knowledge about the spirit, and the one who gets this knowledge is shishya (pupil). The relationship between guru and shishya is special; it is something more than a relationship between the child and the parents, husband and wife, or among friends. Guru is free from egotism. With devotion, without looking for fame or benefits, he guides the pupil towards the ultimate goal. He shows the paths of God and watches the improvements in learning and in the discipline leading him through this path. With love, he awakens trust in him, devotion, discipline, deep understanding, and enlightenment. Believing in his student, the guru does his best for the knowledge to be absorbed. He encourages him to ask questions, learning the truth by analyzing and questioning. The student should have needed qualifications such as high type of awareness and advanced spiritual development; he has to have trust, love, and devotion for his "guru" (Iyengar 2005a: 9-11).

You salute your guru on every occasion, what is seen in all Iyengar's books where he thanks his teachers for all the teaching they did.

The relationship between Arjuna and Krishna described in Bhagavadgita could be here an example of such tradition of parampara (see: 1993).

The role of the teacher is extremely important in the development of an individual. The student has to, according to Iyengar, trust his teacher completely (2008). It is to some extent an idealized concept of a student-master relationship, it is possible if we deal with a real guru and a highly motivated student on the other side. However, this situation does not always look so ideal as Iyengar describes it. Iyengar is very demanding in his descriptions of the ideal guru: "The guru should be confident, challenging, caring, cautious, constructive, and courageous. The clarity and creativity of his teaching should reflect his de- 
votion and dedication to his subject - in this case, the complexities and subtleties of yoga" (Iyengar 2008: 59). However, the disciple should also possess some specific features: "An ideal disciple is obedient, earnest, serious, and always ready to follow the teachings of his or her guru" (ibid.: 59). Both sides should fit each other.

B. K. S. Iyengar also points out that knowledge about yoga cannot be learned during lectures. It is passed on by examples and it is about practical things (Iyengar 2002b: 159-163; 2008). Yoga is thus taught by practice and direct transfer from the teacher. It is very often underlined by Iyengar in many places (2005b: 267). The teacher should know when to be empathic and when to be "ruthless" to the students. He should recognize their capabilities, tiredness, etc. The teacher corrects the poses of the students while they are performing them, touches them. Touch, thus, and corporality are very important elements in yoga teachings. It seems that yoga students are subjected in the corporal dimension to some sort of training, as Marcel Mauss (1973) would state. Discovering and changing the body reactions that were programmed in a different way by socialization requires the teacher's intervention. It seems that there is some sort of secondary socialization of the ways of using the body that can be controlled, not necessarily following its weaknesses and needs in a given moment, for example, the need for rest. You can last in a given pose when the teacher orders so, even though there is an enormous amount of discomfort.

Iyengar claims that the teacher should be decisive and have a positive attitude. Internally, however, he should undergo a critical reflection at all times. Yoga teachers should have respect for their students and the relationship with the students has a character of a master-student type. The teacher should remember about that that all students standing in front of him are as important as he is (Iyengar 2002b: 167). These duty categories expressed by guru in one of the books are the manifestation of his school and are taken into consideration by his yoga students/teachers and used in real practice in hatha-yoga schools. Another yoga guru, Sri Pattabhi Jois, points out that the condition for gaining health through yoga practice is exercising under the watchful eye of the teacher (satguru):

To learn the method for the vinyasas, rechaka and puraka, the bandhas, dhyana, and for trataka [gazing], and the like, one should be certain to consult a Satguru, for it would be wrong to try to learn yoga without recourse to such a teacher. If, however, one follows the scriptural path, and brings it into practice under the guidance of a Satguru who is not only well-versed in the yoga shastra but who has brought it into practice himself, then the three-fold diseases will be destroyed, and one will live a healthy life (2010: 40). 
However, in reality, choosing a teacher is more prosaic, he/she should be chosen taking into consideration also financial criteria and relational ones. Recommendations from friends, healthcare and fitness specialists could be very helpful (see Claire 2004: 20).

\subsection{Teacher-student interactions}

In the initial phase of practicing yoga the teacher's help is required so he could correct the mistakes and body poses. The teacher in a yoga center usually circulates during class among the students and pays attention to the mistakes. Sometimes he praises the practitioners (data from observation), but this rarely happens. One of the practitioners quoted below mentions that at some point while exercising there is a need for a teacher. A yoga instructor that the researcher went to also mentioned at one point the need for the teacher (data from participant observation). The coined phrase "the need for the teacher" could have been thus assimilated from the conversations with the yoga teacher/ teachers, or from the books. The appearance of such need causes consideration of possibility of signing up for group classes and making the decision of starting the practice. There can also be the process of a careful choice of a teacher. ${ }^{1}$ The teacher becomes useful to correct mistakes that the practitioner himself could not notice while working independently:

Initially, I practiced yoga for several months rather regularly, not using specialized help, but the longer I exercised, the stronger need I felt to use some help of an experienced teacher and more often I thought about signing up to school for group classes, mainly so I could obtain correction because you still have to do the whole work yourself. Exercising, the person is not able to tell whether his body positioning is correct, especially that the more you get involved in these exercises, the more details you find in them that, as it turns out, have a vast meaning for the whole and here a person from outside is useful, a person that knows things, that is competent enough to make some corrections, and knowing what to correct, you can work independently at home, paying attention to these points where there were mistakes. (practitioner for 8 years)

1 There are some guidelines for choosing a teacher. Very important is to check his/ her professional affiliations: "Many yoga teachers join professional associations which maintain minimum standards of training and experience, and require members to adhere to a code of ethical conduct" (Claire 2004: 19-20). 


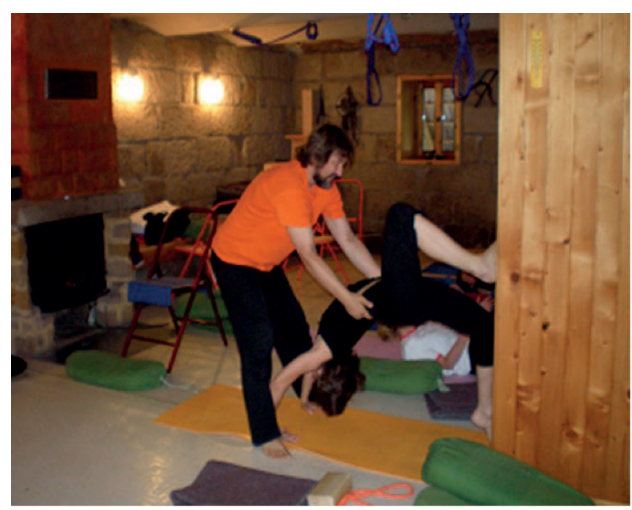

Fig. 16. Correction of mistakes by the teacher, practice during a retreat in Pasterka, teacher: Michał Szczepanik from Hatha-Yoga Center in Łódź (photo by Krzysztof T. Konecki)
An experienced yoga instructor often used to talk about "the need for the teacher" that appears in the practitioner. According to him, the need appeared spontaneously in an appropriate moment. The teacher appears somewhat himself: "There is a need, there is a teacher" (data from participant observation).

Practicing at home, the practitioner has no possibility to obtain remarks from the teacher:

In general, I do not regret that I decided on these classes because it is really different. When I did something incorrectly at home, then no one could tell me that, tell me what I did wrong or just simply come up to me and help me. Now I know that I made some mistakes (laughter). (practitioner for a year)

Sometimes there is a change in teachers. Practitioners might not accept in the beginning some teacher and keeps on looking. The practitioner underlines that teacher's professionalism is important and hatha-yoga students want to practice with such a teacher:

And sometimes you go to a so-called good school and the teachers there will be only thinking about doing the class and that is all. And it is not about that. It is important that the one who takes care of you was professional, that he really knew what to do and knew how to help. I am lucky. (practitioner for 4 years, age 23)

The teachers are also necessary so the practitioner would not hurt himself, which can happen during individual practice:

This teacher, however, pays attention so we would not hurt ourselves. Because you can hurt yourself. (practitioner for 8 years)

Lack of a teacher can cause us to learn how to do some asanas incorrectly, and it can stay with us for long:

You need to approach this with some prudence so you would learn correctly, not to repeat mistakes because if someone learns the mistakes, we had such a gentleman here, he was about 70 years old and he was able to do the lotus, bend himself in any 
direction, he was very fit, but he was unable to do these basic moves because he simply learned them somewhere incorrectly, so when it comes to yoga, I believe that you should learn with a teacher, for sure up to some stage. I do not support such courses publicized somewhere or even on the Internet because it is needed that the instructor come up, touch, explain because each body feels different and apart from that, with any activity you can always harm yourself (practitioner for 2.5 years)

Professor L. Kulmatycki, an expert in yoga practice, claims that harm can also be done by an incompetent teacher:

- What are the dangers of practicing with a bad teacher?

- It can cause more dangers than benefits. If the student has joint degeneration or disk displacement, then some exercises are forbidden. You cannot practice so-called reverse poses with high blood pressure, sinus problems, or with eyes, for example, retina peeling off. With the stomach or duodenal ulcers you are not allowed to perform asanas that increase the pressure in the stomach cavity. People with depression or schizophrenia should limit themselves to stretching and breathing exercises without meditation, without "running inside." A good teacher talks in the beginning with the student about potential illnesses and designs individual exercises. Yoga is not the same for everybody (Augustyn 2008; see also Kulmatycki, Burzyński 2008).

Iyengar also emphasizes that there are incompetent teachers and they can be the source of students' problems. The lack of experience and the lack of patience of students are the main reasons of teachers' problems (Iyengar 2002b: 33).

In hatha-yoga, you use many tools (chairs, wooden blocks, lines, belts, etc.) helping the inexperienced ones to practice so they would not hurt themselves (see fig. 17).

Here is my experience of the contact with the master in an initial phase of learning hatha-yoga:

Today, as usual, I went to yoga class at master Szczepanik's. There was a new exercise, anan-

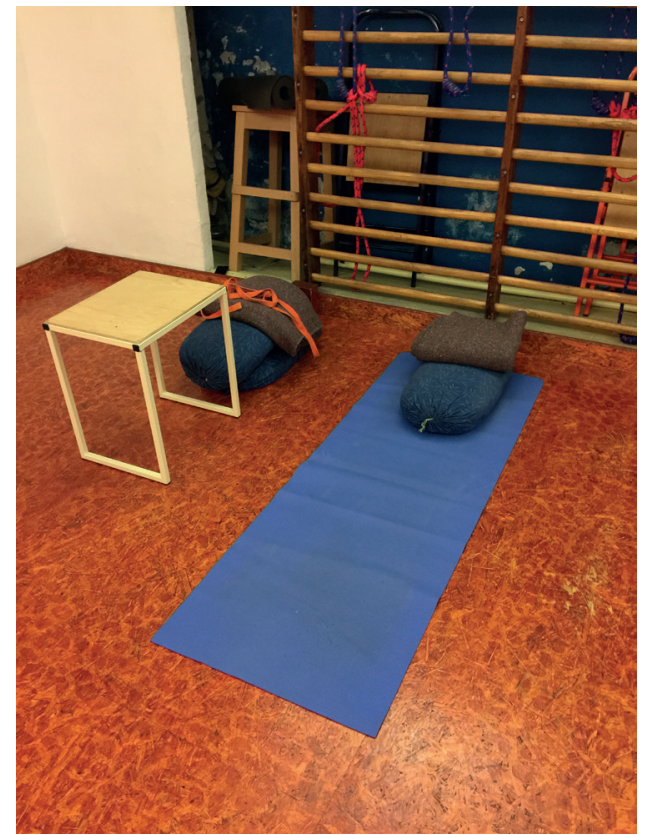

Fig. 17. Tools helping in yoga practice (photo by Krzysztof T. Konecki) 
tasana. ${ }^{2}$ I did it earlier at home based on the photos and books. I noticed that I did not do it correctly. I did not weigh the body correctly and did not position evenly with the side, in a straight line. During the class the trainer said to stretch the body evenly along the side of the mat, put the stretched hand on the hip, and get the balance. Exercises to get the balance were done for both sides (both hips). Only then we caught with the hand the raised up leg, holding it and keeping balance. I had to use the belt so the stretched leg could be straight. I think that without the trainer you can make a lot of mistakes. (5 months of exercises of the author of this work, participant observation and autoethnography, March 28, 2008)

The teacher is also able to understand your mental states and body reactions to the intensive exercises. Very important here is the exchange of experiences not only with the teacher but also with other practitioners:

I am a bit sick today, I feel weak, tired, as if I had a cold (fifth day of intensive exercises, seven hours a day). This is how, according to Michał (evening conversation), the body reacts to an extensive effort in yoga. The body cleanses and there is accompanying pain, anxiety, nausea, joint pain, muscle pain, and other negative effects, even euphoria and depression. We need to know about this and that is why we talk about it, says Michał. (9 months of exercises of the author of this work, participant observation and autoethnography, 11.07.08)

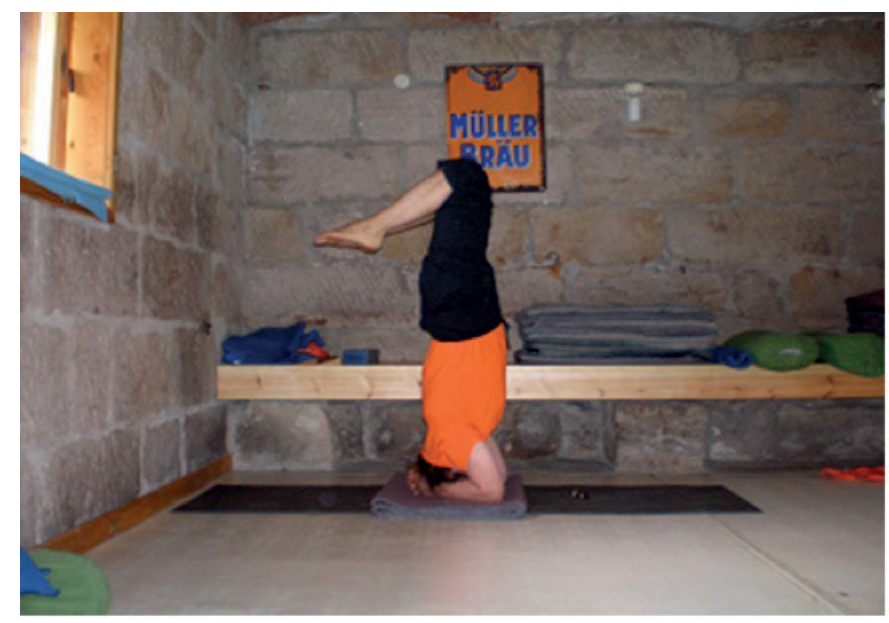

Fig. 18. Practice during field trip in Pasterka, teacher: Michał Szczepanik from Hatha-Yoga Center in Łódź (photo by Krzysztof T. Konecki)
One girl said that pranayama does not convince her. I said that I felt anxiety during pranayama, during deep breathing, that I opened my eyes to come back to this world and calm myself down. Afterwards I stated that I did myself nicely "through yoga to Kochanówek" [common name of a mental asylum in Lodz]. People laughed. The girl said it was normal. I asked Michał how to keep this emancipation and the form acquired here, and

2 See: http://www.ehow.com/video_4954919_anantasana-yoga-pose.html (accessed: 25.07.2014). 
the girl answered for him: exercise, exercise, and exercise. Then she stated that she talks so much after these upward bows today; she whispered that at least an hour a day and it is a lot. (9 months of exercises of the author of this work, participant observation and autoethnography, July 12, 2008)

The exchange of experience among the exercising students points to a social character of yoga and also to a social acquisition of competences to yoga exercises. The teacher is only a part of it, others who exercise are also important in this process:

There are usually conversations with Michał or the practitioners themselves after exercises. They consult the performance of asanas with each other. They show how they do specific asanas. They also tell of their own experiences and what they feel during exercises and how they achieved this point that they are now at. (a year of exercises of the author of this work, participant observation and autoethnography, November 9,2008 )

Understanding of the gestures and the body is possible when we experience the gesture and the body our-

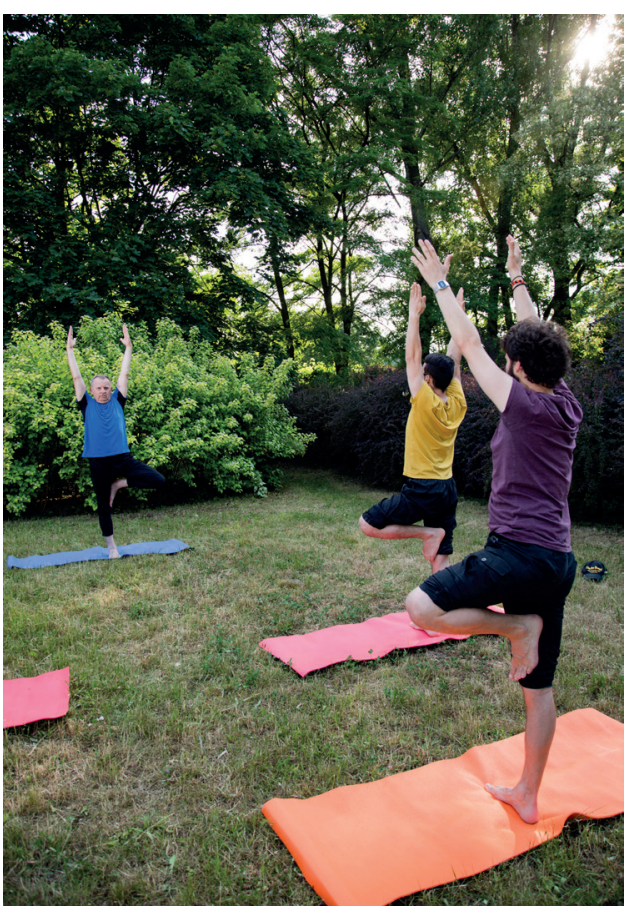

Fig. 19. Giving example by the teacher - following the instructor. Yoga class with Erasmus students, University of Łódź, 2015 (photo by Kamil Głowacki)

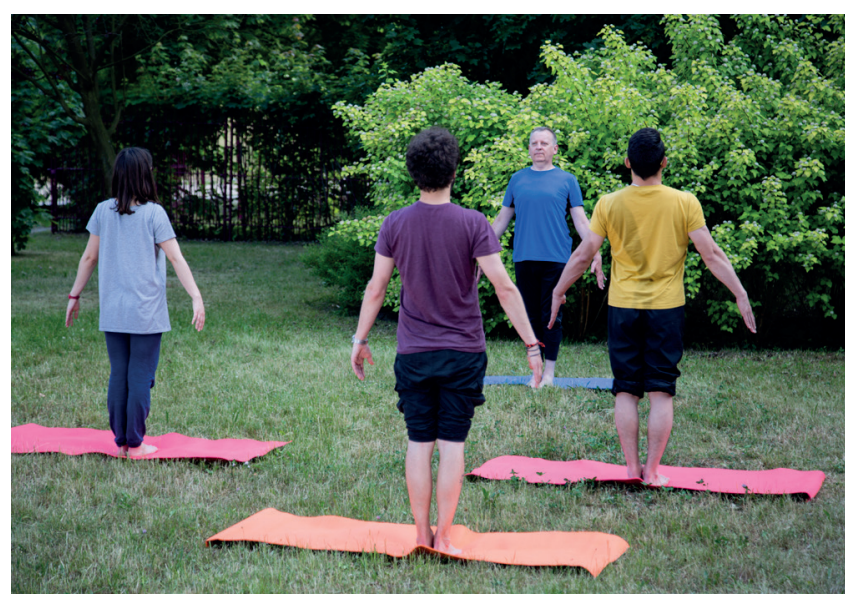

Fig. 20. Giving the example. Yoga class in the park with Erasmus students, University of Łódź, 2015 (photo by Kamil Głowacki) 


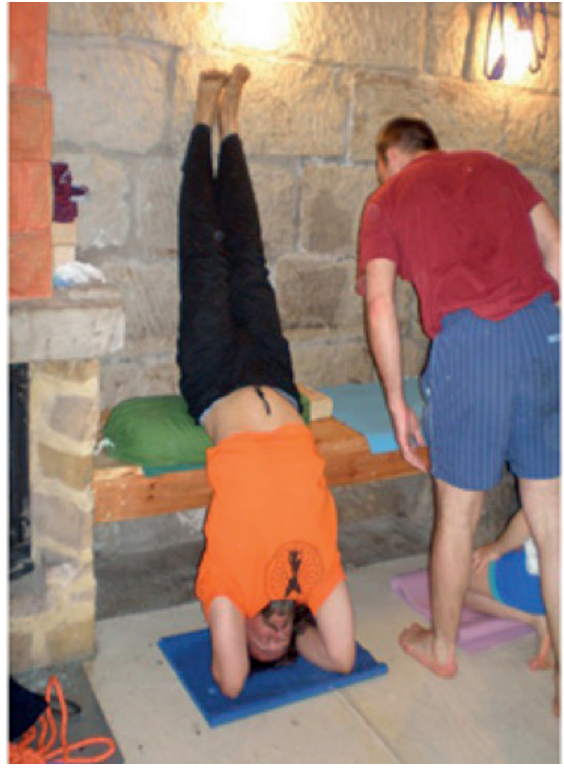

Fig. 21. Direct natural presentation done by a professional yoga teacher, giving example by an instructor (Michał Szczepanik from the Hatha-Yoga Center in Łódź), easier version of standing on the forearms with leaning against the wall. An elementary student carefully observes the master in yoga practice during the trip session in Pasterka,

2008 (photo by Krzysztof T. Konecki) selves. What is more, we are socialized with the usage of the help of others to understand the gesture. The above experience described by me showed me how difficult it is to understand the gesture (yoga position here, asana) taking into consideration the verbal description and the two-dimensional picture. The help of the instructor turns out to be necessary. Exercising on our own we make a lot of mistakes.

Next we did the candle by the wall (sarvangasana) leaning the legs against the wall after a precise measurement of distance from the wall to the belt for the hands. We were supposed to do the candle, but only with the help of the trainer. We were not allowed to do it without his help. It surprised me a bit. We did preparations for this pose by leaning the legs against the wall, something like a plow pose. It was a rather difficult pose because the legs/feet were supposed to go lower and lower and the spine bent more and elongated, as well. ( 5 months of exercises of the author of this work, participant observation and autoethnography, January 26, 2008)

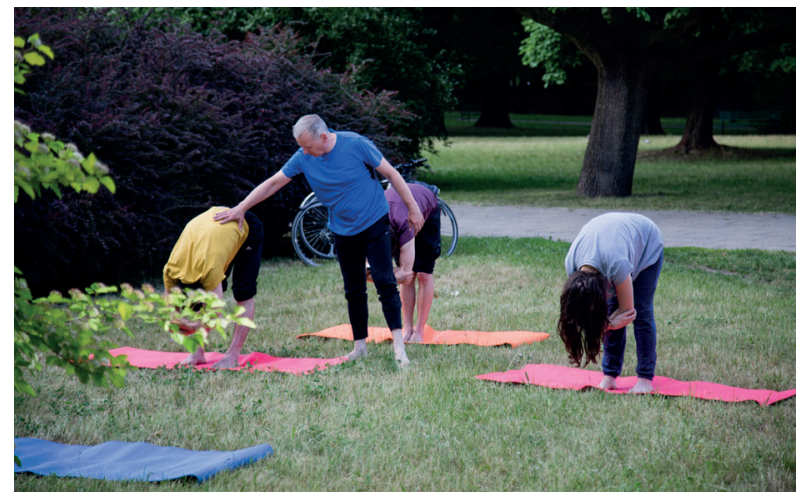

Fig. 22. Corrections by the teacher. Yoga class in the park with Erasmus students, University of Łódź, 2015 (photo by Kamil Głowacki)
As the above example shows, you cannot do the candle pose without the supervision and the help of the instructor. Later, it turned out that it could be dangerous for the neck vertebrae if we do the pose incorrectly.

Practicing yoga on our own, we remember the orders and suggestions of the teacher 
from the yoga class. These instructions imprint in our memory, in our mind, but this is correlated with our body memory (embodiment):

While practicing at home, I hear the voices of the trainers while doing some asanas that were done in the club (e.g., Michał's "maximum!" with different asanas connected with stretching and effort; or "We are pulling it hard to the top!" or with savasana "breath just like it comes out" etc.). During the exercises at the training these words imprint in our body, and while practicing a specific asana, they activate in the memory. I also noticed that at the training, that also other trainers use similarly sounding orders. Probably, they come from the same source. (5 months of exercises of the author of this work, participant observation and autoethnography, February 27, 2008)

I am after a morning pranayama; really, pranayama relaxes. Although during it I sometimes feel tension. I open my eyes to come back to reality and hold on to it and liquidate the tension and anxiety. Michał repeated the same instructions to both pranayamas every single day. To viloma pranayama: "loose, fast breath in, then breathe out till you empty your lungs. Next a breath divided into four, to the navel, lock of the lower ribs, lock up to the nipples, lock up to the upper part of the lungs, lock and next slow, loose and long breath out." I often hear Michał's voice in my head when I do specific exercises on my own. It is also connected with the asanas, I hear, for example, the voice: "maximum, last moments," when you need to hold on in a position in the last moment; "do not drop the leg down," "we go back to the pose," "we do not lay down," etc. (10 months of exercises of the author of this work, participant observation and autoethnography during a retreat in the mountains, July 9, 2008)

\section{Teacher's instructions allow the defining of own feelings:}

Today I asked Michał about this problem with savasana, the problem of the observer that appears when I observe my breath, I mean, this observer appears that observes the observer of the breath. What to do with such thought? Michał said that the thoughts should not be dispersed and held up, let them flow parallel, and on the second track we observe the breath and concentrate on it. The thought passes by, and let them flow, do not stop them. Later, I noticed during savasana that I started observing my thoughts during meditation in this way. Some ran their own course and the other course was observed in the breath. I did not hold up the flowing thoughts as I used to do earlier. (9 months of exercises of the author of this work, participant observation and autoethnography, July 10, 2008)

The above quote shows that the instructor designs the paths of self-observation. The instructor gives grounds for self-observation. Even such subtle things like thoughts flowing during the meditation or savasanas, breath observation, duality of being in savasana are derivatives of instructions. The yoga student would not know how to name some of the psycho phenomena that appear during asana or meditation. How to behave, how to act? The instructor tries to verbally describe these subtle mental processes, and the student can manage with them 
then. The sole instruction "observe your breath" is not understandable for the beginners. Only with practice do questions appear connected with locking the thoughts and controlling them. The instructor helps the student to adjust these problems properly.

The teacher also delivers the rules of the philosophy of yoga practice. For example, the teacher can explain the sportsmen using mainly strength that "if you want to open the body, you need to submit yourself, surrender." In yoga, there is no place for power usage. You cannot open the body using force and athletic effort as those who practice other sports and end up in yoga schools want to do. Yoga is surrendering and understanding of your body and opening it. Taking such suggestions into consideration will surely help to avoid injuries. ${ }^{3}$

What is more, people who want to practice "hard" and ascribe spiritual values to hatha-yoga practice cannot do so without a teacher:

Well, if someone would want to treat yoga like real yogis do, then he has to have a teacher whether he wants it or not, it is spiritual, and so on. But, in my case it is not so, I am not at this stage. (practitioner for 8 years)

We are after singing mantras and meditation. We asked Michał earlier about different things. A young girl asked what the life of yogi is like and how he should act in a normal life. Michał said that he should apply the rule do not harm (ahimsa), do not kill, do not eat meat. He continued on. Food is also an element of yoga. Life can be yoga. Food is important so not to overload the stomach because it disturbs in yoga, not only in asanas. Helping others and working for the benefit of the environment, local surrounding is also yoga. Such work builds relations and they are important. Work without expecting the benefits, judgment, praise is yoga. (9 months of exercises of the author of this work, participant observation and autoethnography, July 10, 2008)

The teacher has a greater charisma and authority if he had experience with great teachers and was in India. A visit in yoga centers in India ennobles the teacher. Then he explains, according to the practitioners, the philosophy of yoga better and shows its connections with everyday life:

Well, in general, Michał often tells us during the class, explains what and how and why (laughter). Michał is my teacher, you know, he went to workshops of Bubicz, he knows him personally, he was in India in Iyengar's Yoga Institute. For him, yoga is something more, that is why he passes on his knowledge to us all, he tells us about the philosophy, that yoga is some sort of a process, you know, exercising the body you exercise the mind, and so on, that, for example, composure, that for sure is needed during exercises, such strength also, that you can transfer this to your everyday life. (practitioner for 7 years)

${ }^{3}$ See:Yoga Practice: Force versus Surrender, http://www.elephantjournal.com/2010/ 05/finding-surrender (accessed: 25.07.2014). 
The teacher's experience is very vital; it is connected with the teacher's age. Teacher's experience is his past fight with own body, "experiencing the body." Experiencing the body is often connected with life experience:

But in this all somehow, you know, I have some limited trust to such young people running the class because they simply did not experience enough of all of that. So what that they do it correctly, but their bodies are still too young to know all about it, well, I don't know if I put it clearly. Well, it is not even about the sense but simply about... for sure they know their bodies less than older people and there is also a different contact with this body, as it seems to me, right. When the body is so young, it surrenders easily to all these changes, right. It is surely different than, as... right, when somebody already has a different life style and the body did not get used to something different yet, there is a different level of work with this body, and I think that such a young person does not have this awareness yet that everybody in a different way... everybody has a different way of contacting this body in different ages and that it is not only on the level of exercises because even if these young people think that this is on the spiritual level, and surely this is how they perceive it, because me, for example, I see it like this, but for me, for example, still, I would not feel, even if I already practiced well at this point. Well, I would not feel competent to run such classes. Really, well, because I think that I have not experienced enough on this path; because this is a longer road, this is not like that. This is not only about exercising the body. In my opinion, well... but, people approach it differently, even if really they only want to exercise their body, then OK, then they run classes and others who want to exercise their bodies also come. But, if you want to look for something more in it, well, then you need to have a bit more experience. (practitioner for approximately 5 years, woman)

Some want to deepen their practice and organize trips to India, to the source where yoga comes from. These are usually yoga instructors or sick people that go to medical or corrective classes. Meeting with the master is a grand experience for yoga teachers. Not all have such an opportunity. Direct contact with the master from India has a great influence on the instructors' attitudes. They can see there that yoga is extremely hard work and a lot of effort is connected with performing asanas. Yoga is supposed to help people, and this message is very vital knowledge for the below quoted interlocutor who went to Puna (to Ramamani Iyengar Memorial Yoga Institute, RIMYI) to learn directly from the yoga masters, members of Iyengar's family:

At the beginning I went once a week, but then it came to me, after several months, that I will write a letter to India asking to accept me to Memorial Yoga Institute because if I am to train, then I would just simply want to see how it is at the source. And it happened that the writing... that with the help of an English teacher that taught my children I simply wrote a letter to Iyengar, in India, in Puna, asking if I could come. And then, after two or three months, my surprise was great when 
I got the answer that if I pay an amount in advance, which was not a small amount at all, then I will get a date in three years time. And, it happened that suddenly this grain became somewhere there such a small plant. Then, when I was sure that I wanted to go there, I started an additional job and started saving money because I had three years for that because, unfortunately, it is not that simple when it comes to finances. And when the time came, and it was the year 2001, I had such a date: June, July. This is the monsoon time there, so I could expect such monsoon weather where the clouds are grey, it rains all the time, and everything smells rotten, so the picture of India that we often have in our heads and hearts turned out to be the worst possible because, well, the weather was... it rained all the time. And I managed to go there. And, the meeting with the Institute changed my whole image I had about yoga because then such real work started. Because the classes are extremely tiring, they are in the morning, in the morning there are several hours of exercise, and in the afternoon there is self-practice, so you spend several hours a day in class. I did not know English well enough so I was left with Sanskrit names, eyes, ears, and attention because the classes are run very strictly. I mean, a moment of inattention causes that the teacher comes and, for example, from a set, there are eighty people in the class and suddenly there is an argument because, for example, somebody does something different than he should, so it really seems in the beginning that it has nothing to do with peace of mind, but then, after years of practice, I know that this teaches how with equal peace cope with sadness and happiness. Then I managed to go in 2006 for the second time, and this second trip was much calmer and more balanced I would say because the first one was really difficult. Because of the tiredness, because of the weather, because of longing, because of Poland being so far away, because these two months were, well, it was extremely difficult, a tiring time for me. I skipped some family issues that changed because of this trip. And what? In 2006, in the Memorial Yoga Institute, I felt really good. I went to Iyengar's children's class that is to Geeta Iyengar and Prashant Iyengar, so... Iyengar runs specific classes because he, himself, does not do the reverse poses, that is, the candle, standing on your head because he had an accident and states that this accident caused that he could open himself up more to people with health issues, thanks to it he learned how to work with such problems. Because in the Institute there is also a medical class that works everyday so sick people from all around the world come there and they achieve relief from the care of different teachers. Often they leave simply walking and come not being completely able to cope with their own bodies. Only that it is really, well, you have to admit that these people need to be very determined and obedient to Iyengar because the classes are really painful, because it is work on the body. They need to have the awareness that this is not something, well, I don't know, like massage, but this is hard work with the body so the muscles straighten the skeleton, and this is the way the skeleton is being straightened. So, this is what it looks like. What else can I say? Iyengar is a unique person and this year he is celebrating his ninetieth birthday and I have never met anyone who would be in such shape at such an age. He is intelligent, good, well, he taught teachers from all over the world that help other people. Even if... even if somebody helps at least one person, then this is some worth, and if you help people, then there is, I think, no greater worth. (certified yoga teacher of B. K. S. Iyengar's method) 
However, there are yoga schools where teachers are also meet with negative opinions. Although, they happen really rarely and the opinions often change to positive ones with the development of the practice:

I was so skeptical at the beginning, I believed that all are set for profit, well, these advertisements and all of this commotion around yoga, I just simply could not make myself, although, in general, the popularity of yoga attracted me. Well, this friend made me. And we went. (practitioner for a year)

\subsection{Conclusions}

The teachers play a vital role in the social world of yoga. This role derives from a traditional yoga where the teacher named guru leads the student through the difficult path of practice. The teacher is deprived of egotism and the student is supposed to fully trust him. All of the modern greats of hatha-yoga mention their teachers and underline their role in their mental and spiritual development. Both B. K. S. Iyengar and Sri Pattabhi Jois, for whom Krishnamacharya was a great guru, emphasize it. It is especially important in a more physically oriented yoga. Then, some exercises should be done under the watchful eye of the guru for safety reasons (Pattabhi 2010: 46-47, 85-86).

The practitioners researched by me underline that currently in the hatha-yoga practice the teacher is supposed to protect the student against making mistakes, and also so the student would not hurt himself (mainly physically). Usually, a teacher with experience is looked for in order to get as much as possible from the classes. And yoga classes are the main place for contact with the teacher who shows and instructs how to do asanas. The modern yoga teacher is a teacher of a physical aspect of yoga, of teaching how to do asanas correctly.

People who look in yoga for spiritual elements (they are in a minority) cannot deal without the teacher because he is inscribed in the structure of this whole philosophy of yoga and he belongs to a typical narration about yoga. Then, the teacher becomes a significant other (a person who is especially important to the practitioner and provides him/him with the perspective of yoga world) (Reynolds, Herman-Kinney 2003: 39, 260) showing the path not only during yoga classes but also outside them. The teacher is then a moral example to follow. She/he delivers new meanings of the world and everyday life. The practitioner evaluates himself/herself from the perspective of significant other (ibid.: 109, 276). 
Search for the teacher can lead the most motivated people even to India. That often happens with people advanced in hatha-yoga practice. Trips to India are often connected not only with the participation in the physical side of yoga but also in the meditation in ashramas (e.g., in Vishwatmak Jangli Maharaj Ashram in Kokamthan) where there is a spiritual development in many practitioners, often confirmed by the practitioners themselves, for example, acquiring a different, new hierarchy of values and discarding values of the modern world of consumerism, calming the mind, feeling happiness, etc. (data from participant observation). The teacher of hatha-yoga can be a very important significant other in changing the life of the practitioner, sometimes as important as parents of yoga claimants. When the interactions with him/her are frequent and the embodiment of contact and instructions is meaningful, the influence could be observable. 


\section{Conclusions}

The issues concerning hatha-yoga can also be looked at from the perspective of the modernization concept that our society has undergone and is still undergoing. Modernization can be understood here as a process of social change concerning industrial and post-industrial economies, increasing the role of the public, lay, and democratic institutions, the expansion of mass-media, increasing wealthiness of the major part of the society and major flexibility in the human physical and social mobility (Lynch 2007: 100).

Emil Durkheim claims that one of the major influences of modernization on the changes in the Western culture was a social increase of the meaning of the individual. The increase of the meaning of the individual and its interests was considered by Durkheim as destabilizing for the society. Lessening of the meaning of the social structure causes the social bonds to fall apart, causes anomy. Acknowledging the individual as the highest value can be the basis of a new, humanistic religion. Durkheim foresaw that news of religiousness would emerge together with collective rituals that would celebrate the individual and keep the cult of the individual alive (see ibid.: 100).

Georg Simmel believed that modernization causes establishing new mystic forms of religion. This new mysticism accented the power of personal, inner experience of an individual, gradually moving away from the traditional forms of religiousness. According to Simmel, even though people where moving away from Christianity, the religious impulses still remained. This new mysticism is, according to him, a new way of life, without any relation to any God. As a result, the whole life is sacred. This way, the life is not devoted to following a God, but to following a specific quality of life, which is feeling the depth and completeness. Simmel was skeptical towards this new mysticism; he did not believe that it is capable of fully expressing new religious life. This mysticism was nothing but a trivial spiritualization of everyday life (see ibid.: 105).

Ernst Troeltsch, talking about the "mystic religion" as a new form of modernity, was thinking about experiencing the "inevitability" and "inner sight" by the individual. He did not connect such experience with ecstatic feelings or visions, but more with personal experience of everyday life, earthliness, as well as completeness and spirituality. However, in his sociological perspective, he connected this type of re- 
ligion with the educated class of Germans that had no financial problems. "Mystic religion" is "a radical religious individualism." The individual chooses from many types of philosophies or religions what suits the best and corresponds with its spiritual experience, and constructs its own perspective of "religiousness." An ideal type of this kind of mystical religion for a specific social group does not have a normative character for a given individual (Hasselle-Newcombe 2005: 316-318).

Ernst Troeltsch believed the same as Georg Simmel, and stated that these new mystical forms of religion develop in the culture-creating groups of artists and intellectualists disappointed with the traditional churches. Troeltsch did not believe that this new form of mysticism was able to build strong interpersonal connections and nets. He believed, just like Simmel did, that this phenomenon is temporary, and as it turned out, they were both wrong (compare Hasselle-Newcombe 2005).

The process of subjectivization in the West - from the times of the above-mentioned classics - continued and still continues on. People chose their lifestyles individually, even through using the help of the commercialism experts. The religious market, giving plenty of options, weakens the traditional religions, as well as its influence on the individual. The process of secularization, according to Peter Berger (1967; see also Kempny, Woroniecka 1999), still continues, although some instances tend to deny this thesis, for example, connected with the situation in our country where the slogan "Polonia semper fidelis" seems to deny the thesis about secularization as a process connected with modernization (Arnold 2012: 212). However, it seems that the continuing secularization can have different faces and advance with a different speed.

In Poland, the individuals often link different beliefs from different religious and philosophical traditions or hold on to contradictory systems of beliefs at the same time. Hatha-yoga is an example of such a hybrid spirituality, and often even religiousness. Other examples are raising belief in fortunetelling and the influence of supernatural powers on our lives (e.g., search and interpretation of signs showing the influence of "providence"), casting a bad spell on someone, strength of talismans, distance telepathy, clairvoyance at the same time declaring participation in some institutionalized religion (Boguszewski 2011: 2). Hence, there could be some sort of erosion of faith and some introduction to secularization as a process moving away from institutionalized religion.

If we look at the modern religions from a culturological point of view, then the concept of the cultural diffusion can explain a lot to us. Religious ideas spread, among other things, thanks to new media, world migration of people; they are interpreted by the individual for its own purposes. Religion on the individual level can have the character 
of a "fusion" type religion. Similarly to the spirit of the fusion cuisine where in the national cuisine spices and ingredients from other national cuisines, often far and exotic countries, are accepted, also the religion can, in a subjective dimension, become a religion of mixed beliefs. Within the frame of one, self-created concept of beliefs the individual accepts different, often contradictory beliefs and rules. According to the research, Poles accept other religions, they are religious Universalists. What is more, religiousness of the Poles (in general the Catholics) does not matter when it comes to believing in fortunetelling and horoscopes, even though such beliefs are contradictory with the teachings of the Church. As a paradox, the higher the index of participation in religious practices, the more the acceptance of statements connected with the para-science, magic, and astrology (Boguszewski 2011). The author of the quantitative research concludes:

The shown analysis points to the complicity of the problem of religiousness and spirituality. It turns out that the life philosophy of Poles is a conglomeration of many, not always coherent, beliefs, also those coming from more popular New Age movement. Recognizing oneself as a devotedly believing person and a frequent attendee in religious practices does not disturb and often even agrees with the belief in magic and astrology and the existence of different types of para-scientific phenomena (Boguszewski 2011: 14).

The declarations of the researched indeed point to the acceptance of many different beliefs and religions by believing Catholics, but from the side of the Church as an institution, there are some reservations against these types of attitudes.

The individuals in the modern world do not thus stop looking for transcendency and understanding of the ultimate concerns. They look for it in other spheres and religions or cultural and philosophical traditions than the ones they were raised in. The process of subjectivization leads to private spiritual searches directed also onto the body of the individual, which can be expressed by the quality of health, mental and emotional well-being, hedonistic feelings, looking for extreme and risky situations (e.g., extreme sports, mountain climbing [see: Kacperczyk 2010a; 2010b]). The Durkheim's "cult of the individual" is the cult of the search for happiness without turning to institutionalized religions and their values. It seems that the motive of the search is the fear of non-existence, fear of death of "I" and body, the last one being, for many people, a habitat of the self. Individual concentration on the body can point to concentration of modern societies on the body, which for some theoreticians means that our societies obtain a somatic character (Turner 1995 as cited in Mullen 2001: 127). 
Connecting the body with the individual in yoga we can obtain some sort of over-usage that stems from sinking the practice in the ego.

"I" is often the source of our problems. "I" lives in our body and we know that the body dies, brain dies, heart stops beating, lungs stop breathing, and the senses feel nothing. So is it not possible, and even highly probable, that the "I" also dies? This is disturbing. If my identity is temporary, ephemeral, is there anything permanent? Is there no stable ground? Our lack of security in this area is, according to yoga, because of its nature, toxic. In order to find own identity, we focus on the aspect of consciousness that lives in our inner body and is called ego. (Iyengar 2005b: 189).

This probable over-usage is a derivative of socialization in our materialistic, commercialized, Western culture:

Inevitably, I say, because our ego consciousness is part of that hungry, seeking, insatiable, frantic world. In other words, we have no firm base. We want to be immortal. We know in our hearts that we are. But we throw it away by misidentifying with all that is perishable (Iyengar 2005b: 189).

The practice of hatha-yoga asanas can also be interpreted in the context of E. Goffman's frame analysis (1974). The practice of asanas is some sort of a frame where the important property is the change of attentiveness points from the everyday life frame to the body and its feelings. The indicator of the change of this orientation is concentration on the body and its parts put differently in different asanas. The change of the frame is marked by initial asanas (supta virasana and balasana, the variant of the child pose with stretched arms) when the individual has to disconnect from everyday life and enter a different reality. These asanas build somewhat a border against the everyday life. The change of the frame means also the change of the space where we do asanas, it is usually separated, quiet, and clean; strangers do not have entrance, it is somewhat a sacrum space for the individual. The practicing person notices the difference comparing new frame with the everyday life. For the advanced practitioners of hatha-yoga, it is important to include the hatha-yoga frame in the everyday life, even though it is difficult, the everyday life of a yogi has a sense of a practice, is a sacrum space, some undividable unity. Adding over the everyday life interpretations coming from practice (constant awareness of the body, control of emotions, perceiving them as passing, discarding connections and cravings, not judging others, distancing from the ego, etc.) allows putting the key of hatha-yoga practice over everyday interactions and changing their interpretations. The primality of some frames decides that some frames are very overbearing for the individu- 
al and their interpretation requires a strong inner look and composure of the new frame so it could be used every day. The hatha-yoga framework in the everyday life is then fully available only for the advanced practitioners (see Chapter 4.3 and Chapter 6 of this book).

Is body a temple of the soul? The question asked in the title of the book (which is a paraphrase of Iyengar's statement that the body is the temple of the soul) brings at least two answers:

1. If the soul of a modern man of the Western culture takes care of herself/himself and his/her ego and its fetishes, then surely the body is the temple of the soul because the whole attention of the Western world concentrates on it. Health, fitness, and psychological peace connected with it, cult of the youth expressed corporally, healthy eating, all of this is connected with the cult of the body and this is where we find places where modern qualities of the Western man are. Eastern gymnastics, martial arts, meditation all inscribe well in the tools used for cultivating the above-mentioned qualities in the body.

2 . The second answer is connected with a more concentrated look at a specific way of achieving of the spirituality in the Western world in spite of the existence of conventional religions. It is a search for deeper transcendental meanings in a corporal practice. Such meanings can be looked for thanks to some esoteric Eastern practices focused on perfecting the body. Hatha-yoga, ashtanga yoga, as well, is such a practice. In this context, the translation of one of Patanjali's sutras by Iyengar perfectly pictures the sense of it: "Asana is a perfect firmness of body, steadiness of intelligence, and benevolence of spirit" (Iyengar 2002a: 149).

Hatha-yoga in the culture and Western society context can be treated as a quasi-religion where some meaning is ascribed to supernatural powers, but this is not religion in a traditional sense, and it is also not in an institutional sense. Quasi-religion in a "normal" phenomenon because it belongs to the group of "boundary" phenomena, and - according to G. Simmel (see Rudy, Greil 1988: 50) - the marginality is some sort of a social form that describes this form of religiousness. It is often difficult to set the border between religion and non-religion. Hatha-yoga, in the Western context, is on the border of sacrum and profanum. You pass here in an accepted way from one universe of discourse to another one without major resistance and discussion. This passing holds a form of a constant "bordering," it is also some sort of a universe of discourse. The resistance among the practitioners in Poland appears when one needs to declare in favor of the religion coming from outside the universe of yoga discourse and non-yoga, and this is when the descriptions are generated to recount new universe 
of discourse based on the phenomena of "bordering" worlds. Although still in this bordering, the individual is in the center of attention since the concentration on oneself is the condition of these searches. Paradoxically, what originally is offered by hatha-yoga, that is, moving away from ego and its annihilation, is often used for its strengthening. It is caused by own subjectivization of the culture and the spirituality of the modern society.

On the individual level, the individual makes autonomic choices of values. The motives of the choice of the practice of yoga mirror somehow values of the modern world where the care of own body has an immense role: health, beauty, mental well-being, control over emotions, self-development, etc. One's beliefs connected with the topic of ultimate concerns are a private issue. Pluralization of modern societies allows a free choice and also a free game of the systems and institutions offering spiritual help (even spiritual service), change of identity, or a set of loose values and beliefs connected with ultimate concerns. The spirituality becomes somewhat privatized and at the same time subjectified in a "free" choice of the individual. Secularization tendencies of the modern world (Berger 1967: 157; see also Knoblauch 2006: 20-22) mirror themselves in this privatization of spirituality and beliefs. Secularization concerns the institutional sphere because on the individual and private level the individual still wants to overcome its biological boundaries and the borders set by the body in order to direct itself towards the spirituality and ultimate concerns or their substitutions, such as individual identity, personal development, or health. This urge to "overcome" points also to some features of para-religion appearing in hatha-yoga. The interests of para-religion are connected with the interest in ultimate and existential concerns in human life, but there is no inclusion of beliefs in supernatural powers.

It needs to be underlined that yoga is independent from Hinduism. It is the other way around: it is the fossilized, ritualized Hinduism that was forced a long time ago - due to the popularity of yoga - to accept many of its techniques, which broke the monopoly of Brahmins to the truth and brought back the people the freedom of personal, spiritual search.

Yoga deals with the whole human, also with its inner sacrum. It shares the field of work partially with religion, but uses completely different methods.

In my thirty long years work as a yoga teacher, I met many people who, thanks to this practice, deepened greatly, understood better, and appreciated more their Christian faith, its truths and holy books. It happened so because yoga strengthens physical, ethical, mental, and spiritual powers thanks to which the human becomes more mobile and straightforward, independent, clean, open, and humble. (Pawłowska 2012, an interview with yoga teacher S. Bubicz). 
Para-religious approaches appear, for example, in some psychotherapies, corporations, or other institutions changing the identity of the individual. The individuals, thanks to the work on the body, overcome their boundaries and identities, go to a different level of understanding oneself and the reality, change their identity. They can observe it retrospectively when they notice that their body changed, that they changed the diet, the perception of the feelings of the mind and the body changed, they achieved greater emotional calmness, observed that others noticed the change in them. All these elements show that the change happened, that the boundaries, both mental and physical, were overcome. There is often a private reflection of a spiritual nature appearing, a reflection connected with the human existence, its changes and persistence. For the individual, hatha-yoga is thus a tool for a change and - at the same time - its essence. When it comes to the tools, then the Western culture gives the individual a lot, however, if hatha-yoga becomes an important element of the everyday life of the individual, then he/she is immersed in it and fully finds there its basis for existence and ultimate interpretation of reality. It has a character of a more "immanent" spirituality rather than "transcendent theology" looking for outer God that is to be feared and loved at the same time.

In any case, the body is here the temple of the soul; in the first instance, soul fully saturated with Western values, in the second instance, enriched by the values of the Hindu culture. The individual in the Western world bends over its body, taking more care of it, using it to its mental or spiritual searches, and often finds there its destiny. Whatever this destiny is, as it turns out, you can find it in your own body. Iyengar says about this corporal objectivization that: "The final, correct asana is a true expression of 'I am That, That is God.' " (Iyengar 2005b: 189). 



\section{Bibliography}

Arnold, Maik (2012). How does religion matter today in Poland? Secularization in Europe and the "causa Polonia semper fidelis." In: M. Arnold, P. Łukasik (eds.), Europe and America in the Mirror. Culture, Economy, and History (pp. 199-238). Kraków: Nomos.

Augustyn, Aneta (2009). Joga na dobre i złe [Yoga for better or for worse]. Gazeta Wyborcza, January 20; http://wyborcza.pl/1,75476,5897236,Joga_na_dobre_i_na_zle.html (accessed: 10.10.2010).

Augustyn, Aneta (2010). Joga to jest to! [Yoga, this is it!]. Gazeta Wyborcza, November 19.

Becker, Howard S. (1982). Art Worlds. Berkeley: University of California Press.

Bennett, Bija (2002). Emotional Yoga. How the Body Can Heal the Mind. New York: Fireside.

Berger, Peter (1967). The Sacred Canopy: Elements of Sociological Theory of Religion. New York: Anchor Books.

Berger, Peter and Thomas Luckmann (1991). The Social Construction of Reality. A Treatise in the Sociology of Knowledge. Harmondsworth: Penguin Books.

Bhagavadgita (1993). Transl. by Sir Edwin Arnold. Mineola, NY: Dover Publications.

Bogunia-Borowska Małgorzata and Piotr Sztompka (2012). Fotospołeczeństwo. Antologia tekstów z socjologii wizualnej [Photosociety. Anthology of visual sociology texts]. Kraków: Znak.

Boguszewski, Rafał (2010). Co jest ważne, co można, a czego nie wolno - normy i wartości w życiu Polaków [What is important, what is allowed and what is not - norms and values in the lives of Polish people]; http://www.cbos.pl/SPISKOM. POL/2010/K 099 10.PDF (accessed: 25.07.2014).

Boguszewski, Rafał (2011). Polacy wobec niektórych poglądów z kręgu New Age [Polish People towards some New Age ideas]; http://www.cbos.pl/SPISKOM. POL/2011/K 135_11.PDF (accessed: 25.07.2014).

Bragdon, Claude Fayette (1943). Yoga for You. New York: Alfred A. Knopf.

Bragdon, Claude Fayette (1970). Joga dla ciebie, czytelniku [Yoga for You, Reader]. Transl. by W. Dynowska. Bombay: Biblioteka Polsko-Indyjska.

Brandt, Keri (2006). Intelligent bodies: Embodied subjectivity human-horse communication. In: D. Waskul, P. Vannini (eds.), Body/Embodiment. Symbolic Interaction and the Sociology of the Body (pp. 141-152). Hampshire: Ashgate.

Budilovsky, Joan, Eve Adamson and Carolyn Flynn (2007). Joga dla żółtodziobów czyli wszystko co powinieneś wiedzieć o... [The Complete Idiot's Guide to Yoga]. Transl. by A. Zdziemborska. Warsaw: Rebis.

Claire, Thomas (2004). Yoga For Men. Posture for Healthy, Stress-Free Living. New York: New Page Books.

Clarke, Adele E. (1991). Social worlds/arenas theory as organizational theory. In: D. R. Maines (ed.), Social Organization and Social Process. Essays in Honor of Anselm Strauss (pp. 119-158). New York: Aldine de Gruyter.

Cyboran, Leon (1973). Filozofia jogi - próba nowej interpretacji [Philosophy of Yoga - Attempt of Another Interpretation]. Warsaw: PWN. 
Cyboran, Leon (1986). Klasyczna joga indyjska. Jogasutry przypisywane Patańdżalemu i Jogabhaszja, czyli komentarz do Jogasutr przypisywany Wjasie [Classic Indian Yoga]. Warsaw: PWN.

Davie, Grace (2007). The Sociology of Religion. London: Sage.

De Michelis, Elisabeth (2008). Modern yoga: History and forms. In: M. Singleton, J. Byrne (eds.), Yoga in the Modern World. Contemporary Perspectives (pp. 17-35). New York: Routledge.

Dewey, John (1931). Qualitative thought. In: Philosophy and Civilization (pp. 93-116). New York: Minton, Balch \& Company.

Dobroch, Bartosz (2008). Bezpieczniej niż w aucie [Safer than in your car]. Tygodnik Powszechny 12 (3063); http://tygodnik.onet.pl/37,0,7758,3,artykul.html (accessed: 23.03.2008).

Drozdowski, Rafał (2008). Autodefiniujące fotopamiątki [Autodefining photo-souvenirs]. In: R. Drozdowski, M. Krajewski, Wyobraźnia społeczna. Horyzonty, źródła, dynami$k a$ [Social Imagination. Horizons, sources, dynamics]. Poznań: Wydawnictwo UAM.

Drozdowski, Rafał and Marek Krajewski (2008). Wyobraźnia społeczna. Horyzonty, źródła, dynamika [Social Imagination. Horizons, sources, dynamics]. Poznań: Wydawnictwo UAM.

Eliade, Mircea (1997). Joga. Nieśmiertelność i wolność [Le Yoga. Immortalité et Liberté]. Transl. by B. Baranowski. Warsaw: PWN.

Eliade, Mircea (2004). Patańdżali i joga [Patañjali et le yoga]. Transl. by I. Kania. Warsaw: Wydawnictwo KR.

Eliade, Mircea (2009). Techniki jogi [Techniques du yoga]. Transl. by B. Biały. Warsaw: Aletheia.

Garbara, Małgorzata and Janusz Szopa (2013). Asany jogi dla współczesnego człowieka [Yoga asanas for modern man]. Katowice: Wydawnictwo Akademii Wychowania Fizycznego im. Jerzego Kukuczki.

Ghose, Aurobindo Śri (1962). Joga i co w niej pomaga [The Synthesis of Yoga]. Transl. by W. Dynowska. Madras: Biblioteka Polsko-Indyjska.

Goffman, Erving (1974). Frame Analysis: An Essay on the Organization of Experience. New York: Harper \& Row.

Górna, Joanna and Janusz Szopa (2005). Joga. Ruch, oddech, relaks. [Yoga. Physical movement, breathing, relaxation.]. Katowice: Kos.

Górski, Sebastian (2004). Joga krzepi [Yoga invigorates]. Wprost 8; http://www.wprost. pl/ar/56385/Joga-krzepi (accessed: 26.07.2014).

Greil, Arthur L. and David Rudy (1984). Social cocoons: Encapsulation and identity transformation organizations. Sociological Inquiry 54: 260-278.

Greil, Arthur L. and Thomas Robbins (eds.) (1994). Between the Sacred and the Secular. Greenwich: JAI Press.

Hasselle-Newcombe, Suzanne (2005). Spirituality and "mystical religion" in contemporary society: A case study of British practitioners of the Iyengar method of yoga. Journal of Contemporary Religion 20 (3): 305-321.

Heath, Christian and Paul Luff (2007a). Ordering competition: The interactional accomplishment of the sale of fine art and antiques at auction. British Journal of Sociology 58 (1): 63-85.

Heath, Christian and Paul Luff (2007b). Gesture and institutional interaction: Figuring bids in auctions of fine art and antiques. Gesture 7 (2): 215-241.

Heelas, Paul L. F. (2000). Expressive spirituality and humanistic expressivism: Sources of significance beyond church and chapel. In: S. Sutcliffe, M. Bowman (ed.), Beyond 
the New Age: Exploring Alternative Spirituality (pp. 237-254). Edinburgh: Edinburgh University Press.

Heelas, Paul, Linda Woodhead, Benjamin Seel, Bronislaw Szerszynski, and Karin Tusting (2004). The Spiritual Revolution: Why Religion is Giving Way to Spirituality. Oxford: Blackwell.

Hochschild, Arlie (1983). The Managed Heart: The Commercialization of Human Feeling. Berkeley: University of California Press.

Iyengar, B. K. S. (1983). Light on Pranayama. Pranayama Dipika. London: Unwin Paperbacks.

Iyengar, B. K. S. (2002a). Lights of the Yoga Sutras of Patanjali. London-San Francisco: Thorsons.

Iyengar, B. K. S. (2002b). The Tree of Yoga. Boston: Shambhala.

Iyengar, B. K. S. (2005a). The Illustrated Light on Yoga. New Delhi: HarperCollins Publishers India \& The India Today Group.

Iyengar, B. K. S. (2005b). Light on Life. The Yoga Journey to Wholeness, Inner Peace, and Ultimate Freedom. Pennsylvania: Rodale.

Iyengar, B. K. S. (2008). Yoga: the Path to Holistic Health. New York: Dorling Kindersley.

Iyengar, B. K. S. (n.d.). Światło jogi [The Light on Yoga]. Transl. by S. Bubicz. Warsaw: Virya.

Jakubczak, Marzenna (1999). Sankhja i joga: podstawy filozofii Patańdżalego [Sankhya and Yoga: The Rudiments of Patanjali's Philosophy]. Kraków: Wydawnictwo Oddziału Polskiej Akademii Nauk.

Jakubowska, Honorata (2009). Socjologia ciała [Sociology of the Body]. Poznań: Wydawnictwo Naukowe UAM.

Jaros, Ewa (2011). Joga dla kobiet w ciąży [Yoga for Pregnant Women]. Warsaw: Świat Książki.

Javalgekar, Ravi (2008). Joga lecznicza. Asany na różne choroby [Therapeutic Yoga]. Transl. by A. Wolnicka. Łódź: Ravi.

Jung, Carl G. (2003). Psychologia kundalini-jogi. Według notatek z seminariów (1932) [The Psychology of Kundalini Yoga: Notes of the Seminar Given in 1932]. Transl. by R. Reszke. Warsaw: Wydawnictwo KR.

Kacperczyk, Anna (2005). Zastosowanie koncepcji społecznych światów w badaniach empirycznych [The concept of social worlds applied to empirical study]. In: E. Hałas, K. T. Konecki (eds.), Konstruowanie jaźni i społeczeństwa. Europejskie warianty interakcjonizmu symbolicznego (pp. 169-191). Warsaw: Scholar.

Kacperczyk, Anna (2010a). Wykorzystanie nagrań wideo w mikroanalizie aktywności wspinaczkowej. Wizualna teoria ugruntowana na przykładzie bulderingu [The use of video recordings in the microanalysis of climbing activity. Visual grounded theory with regard to bouldering]. Paper presented at the $14^{\text {th }}$ Sociological Congress in Kraków.

Kacperczyk, Anna (2010b). “Ludzie gór”. Procesy tożsamościowe uczestników społecznego świata w konfrontacji z przyrodą nieożywioną [“Mountain’s People.” Identity processes of social world participants in confrontation with inanimate nature]. In: K. T. Konecki, A. Kacperczyk (eds.), Procesy tożsamościowe. Symboliczno-interakcyjny wymiar konstruowania ładu i nieładu społecznego (pp. 165-178). Łódź: Wydawnictwo Uniwersytetu Łódzkiego.

Kempny, Marian and Grażyna Woroniecka (eds.) (1999). Religia i kultura w globalizujacym się świecie [Religion and Culture in the Globalizing World]. Kraków: Nomos. 
Knoblauch, Hubert (2006). "Niewidzialna religia" Thomasa Luckmanna, czyli o przemianie religii w religijność [Thomas Luckmann's "invisible religion." How religion becomes religiosity]. In: T. Luckmann, Niewidzialna religia (pp. 37-76). Kraków: Nomos.

Knoblauch, Hubert (2008). The performance of knowledge: Pointing and knowledge in PowerPoint presentations. Cultural Sociology 2 (1): 75-97.

Kogler, Aladár (1975). Joga: podstawy ćwiczeń fizycznych [Yoga: Basic Principles of Physical Activity]. Transl. by H. Janaszek-Ivaničková. Warsaw-Bratislava: Sport, Słowackie Wydawnictwo Wychowania Fizycznego.

Konecki, Krzysztof T. (2008). Touching and gesture exchange as an element of emotional bond construction. Application of visual sociology in the research on interaction between humans and animals. Forum Qualitative Sozialforschung/Forum: Qualitative Social Research 9 (3), art. 33; http://nbn-resolving.de/urn:nbn:de:0114-fqs0803337 (accessed: 10.03.2010).

Konecki, Krzysztof T. (2009). Teaching visual grounded theory. Qualitative Sociology Review 5 (3): 64-92; http://www.qualitativesociologyreview.org/ENG/Volume14/ QSR_5_3_Konecki.pdf (accessed: 10.03.2010).

Konecki, Krzysztof T. (2010). Wywiad na podstawie filmów w badaniach praktyki jogi - na przykładzie badań cielesności i gestów w społecznym świecie praktyki jogi [Movie-elicited interviews in yoga practice research - the example of research on embodiment and gestures in the social world of yoga practice]. In: J. Leoński, M. Fiternicka-Gorzko (eds.), Kultury, subkultury i światy społeczne w badaniach jakościowych (pp. 303-335). Szczecin: Volumina.pl.

Konecki, Krzysztof T. (2011a). Book review: Yoga body. The origins of modern posture practice by Marc Singleton. Qualitative Sociology Review 7 (3): 97-101; http://www. qualitativesociologyreview.org/ENG/Volume20/QSR_7_3_Konecki.pdf (accessed: 20.07.2014).

Konecki, Krzysztof T. (2011b). Visual grounded theory: A methodological outline and examples from empirical work. Revija za sociologiju 41 (2): 131-160.

Konecki, Krzysztof T. (2012). Ciało świątynią duszy, czyli o procesie budowania tożsamości praktykującego hatha-jogę. Konstruowanie prywatnej quasi-religii [Body, the temple of the soul - the process of building the identity of hatha-yoga practitioner. The construction of private quasi-religion]. Przegląd Socjologii Jakościowej 8 (2): 64-111; http://www.qualitativesociologyreview.org/PL/Volume19/PSJ_8_2_Konecki. pdf (accessed: 25.07.2014).

Konecki, Krzysztof T. and Piotr Chomczyński (2012). Słownik socjologii jakościowej [The Dictionary of Qualitative Sociology]. Warsaw: Difin.

Kulmatycki, Lesław (1993). Stres, joga, relaksacja: podręcznik ćwiczeń [Stress, Yoga, Relaxation. Textbook]. Wrocław: Wacław Bagiński i Synowie.

Kulmatycki, Lesław (1999). Stres i joga [Stress and Yoga]. Warsaw: Książka i Wiedza.

Kulmatycki, Lesław (2000). Joga dla zdrowia: podręcznik ćwiczeń [Yoga for Your Health. Textbook]. Warsaw: Książka i Wiedza.

Kulmatycki, Lesław and Zdzisław Burzyński (1999). Opinie studentów na temat ćwiczeń jogi [Students' opinions on yoga practice]. In: K. Zatoń (ed.), Problemy kultury fizycznej w badaniach naukowych. Wrocław: Wydawnictwo AWF.

Kulmatycki, Lesław and Zdzisław Burzyński (2008). Kto uczy hatha jogi w Polsce? [Who teaches hatha-yoga in Poland?]. Kwartalnik Pedagogiczny 2: 157-172.

Lichy, Xanath (2004). Widzieć jasno: gimnastyka i joga dla oczu, harmonizacja za pomoca. kolorów, relaksacja, lepszy wzrok bez okularów [Voir Clair: Comment abandonner ses lunettes]. Transl. by J. Józefowicz-Pacuła. Warsaw: Klub dla Ciebie. 
Laurier, Eric, Ramia Maze and Johan Lundin (2006). Putting the dog back in the park: Animal and human mind-in-action. Mind, Culture \& Activity 13: 2-24.

Luckmann, Thomas (1967). The Invisible Religion. New York: Macmillan.

Luckmann, Thomas (2006). Niewidzialna religia [The Invisible Religion]. Transl. by L. Bluszcz. Kraków: Nomos.

Lutosławski, Wincenty (1909). Rozwój potęgi woli przez psychofizyczne ćwiczenia według dawnych aryjskich tradycji oraz własnych swoich doświadczeń podaje do użytku rodaków... [The Development of Will's Might Through Psychophysical Exercises According to Ancient Aryan Traditions and Own Experiences I Give to My Fellow Countrymen...]. Warsaw: Gebethner \& Wolff.

Lynch, Gordon (2007). The New Spirituality. An Introduction to Progressive Belief in Twenty-First Century. London: I. B. Tauris.

Maddern, Jan (2005). Odchudzająca joga [Yoga Burns Fat]. Transl. by M. Cegieła. Warsaw: Klub dla Ciebie.

Magala, Sławomir (2009). The Management of Meaning in Organizations. Basingstoke: Palgrave Macmillan.

Martínez Nussio, Estefanía (2009). Joga dla kobiet [Yoga for Women]. Transl. by M. Adamczyk. Warsaw: Bellona.

Matus, Thomas (2003). Joga i modlitwa Jezusowa: doświadczenie w wierze [Yoga and the Jesus Prayer]. Transl. by J. Sar. Kraków: Homini.

Mauss, Marcel (1973). Techniques of the body. Economy and Society 2: 70-88.

Michalska, Malina (1974). Hatha-joga dla wszystkich [Hatha-Yoga for Everyone]. Warsaw: Państwowy Zakład Wydawnictw Lekarskich.

Mills, C. Wright (1940). Situated actions and vocabularies of motive. American Sociological Review 5: 904-913.

Moćko, Justyna (2007). Joga, książki, wydawnictwo - rozmowa ze Sławomirem Bubiczem [Yoga, Books, Publishing House - A Conversation with Sławomir Bubicz]; http:// www.joga-joga.pl/pl128/teksty386/joga_ksiazki_wydawnictwo_8211_rozmowa_ze_ slawom (accessed: 26.07.2014).

Mullen, Kenneth (2001). Pleasing to behold: Healing and the visualized body. Mental Health, Religion \& Culture 4 (2): 119-132.

Nagarathna, Raghuram, Robin Monro and H. R. Nagendra, Vivekananda Yoga Therapy and Research Foundation (1990). Yoga for Common Ailments. London: Gaia Books.

Nelke, Magdalena (2010). Dynamicznie i z optymizmem - branża fitness w Polsce [Dynamically and with optimism - fitness business in Poland]. body LIFE 4; http://www.fit. $\mathrm{pl} / \mathrm{g} / \mathrm{str} / \mathrm{file} /$ ankieta_fitness.pdf (accessed: 4.02.2011).

Niedźwiedź, Sabina (1997). Joga: oddech wolności [Yoga: The Breath of Freedom]. Wrocław: Alma.

Pagis, Michal (2010a). From abstract concepts to experiential knowledge: Embodying enlightenment in a meditation center. Qualitative Sociology 33: 469-489.

Pagis, Michal (2010b). Producing intersubjectivity in silence: An ethnographic study of meditation practice. Ethnography 11: 309-328.

Pattabhi Jois, Sri (2010). Yoga Mala: The Original Teachings of Ashtanga Yoga Master. New York: North Point Press.

Pattabhi Jois, Sri (n.d.). Joga Mala [Yoga Mala]. Warsaw: Virya.

Pawłowska, Marta (2012). Pionier jogi w Polsce: Jogini na swojej drodze spotykają czasem "tajemne" zjawiska [The Yoga Pioneer in Poland: Sometimes Yogis Encounter on Their Path the "Mysterious" Events"]. An Interview with Sławomir Bubicz; http:// natemat.pl/10835, pionier-jogi-w-polsce-jogini-na-swojej-drodze-spotykaja-czasem-tajemne-zjawiska (accessed: 27.05.2012). 
Pilkington, Maya (1995). Stare drogi do zdrowia na nowo: akupunktura, shiatsu, terapia reflektoryczna, masaż tkanki łącznej, ziołolecznictwo, terapia Bacha, bioenergetyka, hipnoza, rebirthing, joga, makrobiotyka, wizualizacja, leczenie światłem, dźwiękiem i sztuka [Alternative Healing and Your Health]. Transl. by D. Łyżnik. Warsaw: Oficyna Wydawnicza Spar.

Prus, Robert (1996). Symbolic Interaction and Ethnographic Research: Intersubjectivity and the Study of Human Lived Experience. Albany-New York: State University of New York Press.

Prus, Robert (2009). Reconceptualizing the study of community life: Emile Durkheim's "Pragmatism and Sociology." The American Sociologist 40: 106-146.

Pūrnā, Svāmi (1996). Joga dla ciała i umysłu: 12-tygodniowy program ćwiczeń fizycznych i medytacji [Yoga for the Body and for the Mind: The 12-Week Program of Exercises and Meditation]. Transl. by M. Czekański. Warsaw: Agencja Wydawnicza "Comes" Lidia i Andrzej Skarżyńscy.

Ramacharaka, Yogi (1922). Hatha-joga: nauka jogów o zdrowiu fizycznym i o sztuce oddychania [Science of Breath: A Complete Manual of the Oriental Breathing Philosophy of Physical, Mental, Psychic, and Spiritual Development]. Transl. by A. Lange. Warsaw: Trzaska, Evert i Michalski.

Ramacharaka, Yogi (1923). Drogi dojścia jogów indyjskich [Advanced Course in Yogi Philosophy and Oriental Occultism]. Transl. by A. Lange. Warsaw: Trzaska, Evert i Michalski.

Ramacharaka, Yogi (1930). Dżnani-joga: joga mądrości: książka o najwyższych naukach jogów/Jogi Rama-Czaraka [Lessons in Gnani Yoga (The Yoga of Wisdom)]. Transl. by W. Dunin-Goździkowski. Warsaw: Trzaska, Evert i Michalski.

Reignier, Jude (2010). Joga na co dzień [Easy Yoga]. Transl. by M. Machnik. Gliwice: Helion.

Reynolds, Larry T. and Nancy J. Herman-Kinney (2003). Handbook of Symbolic Interactionism. Walnut Creek: AltaMira Press.

Rokita, Zofia (2008a). Radość z jogi [The Joy of Yoga]. Rzeczpospolita; http://www.rp.pl/ artykul/220001.html (accessed: 4.02.2011).

Rokita, Zofia (2008b). Skręty są dobre na umysł [Twists Are Good for the Mind]. Rzeczpospolita; http://www.rp.pl/artykul/215866.html (accessed: 4.02.2011).

Rudy, David, R. and Arthur R. Greil (1988). Is Alcoholics Anonymous a religious organization? Meditations on marginality. Sociology of Religion 50 (1): 41-51.

Scheff, Thomas (1990). Microsociology. Discourse, Emotion, and Social Structure. Chicago-London: Chicago University Press.

Scherer, Burkhard (2009). Buddyzm [Buddhism]. Transl. by G. Kuśnierz. Kęty: Wydawnictwo Marek Derewiecki.

Schnettler, Bernt (2008). W stronę socjologii wiedzy wizualnej [Towards a Sociology of Visual Knowledge]. Przegląd Socjologii Jakościowej 4 (3): 116-142; http:// www.qualitativesociologyreview.org/PL/Volume8/PSJ_4_3_Schnettler.pdf (accessed: 26.07.2014).

Schrock, Douglas and Emily Boyd (2006). Reflexive transembodiment. In: D. Waskul, P. Vannini (eds.), Body/Embodiment. Symbolic Interaction and the Sociology of the Body (pp. 51-68). Hampshire: Ashgate.

Schubert, Cornelius (2006). Video analysis of practice and the practice of video analysis. Selecting field and focus in videography. In: H. Knoblauch, B. Schnettler, J. Raab, H. G. Soeffner (eds.), Video Analysis: Methodology and Methods. Qualitative Audiovisual Data Analysis in Sociology (pp. 115-126). Frankfurt am Main: Peter Lang. 
Schüle, Christian (2007). W szponach lęku. Piekło białych kołnierzyków [In the clutches of fear. White collars' hell]. Tygodnik Forum 45; http://www.polityka.pl/tygodnikpolityka/swiat/234646,1,w-szponach-leku.read (accessed: 2.08.2011).

Seung, Sahn (2006). Strzepując popiół na Buddę [Dropping Ashes on the Buddha]. Transl. by J. Szczepan, A. Szostka. Warsaw: Szkoła Zen Kwan Um.

Shott, Susan (1979). Emotion and social life: A symbolic interactionist analysis. American Journal of Sociology 84: 1317-1334.

Singleton, Marc (2010a). Yoga Body. The Origin of Modern Posture Practice. Oxford: Oxford University Press.

Singleton, Marc (2010b). Translations, belief frameworks and modern yoga practice. The Magazine of Yoga; http://themagazineofyoga.com/blog/2010/10/12/conversation-mark-singleton (accessed: 4.09.2011).

Singleton, Marc (2010c). Yoga's greater truth. Yoga Journal; http://www.yogajournal. com/wisdom/2610 (accessed: 3.09.2011).

Singleton, Marc (2013). Transnational exchange and the genesis of modern postural yoga. In: B. Hauser (ed.), Yoga Travelling. Bodily Practice in Transcultural Perspective (pp. 37-56). Heidelberg-New York-Dordrecht-London: Springer.

Sjoman, Norman (1996). The Yoga Tradition of the Mysore Palace. New Delhi: Abhinav Publications.

Star, Susan L. and James R. Griesemer (1989). Institutional ecology, "translations," and boundary objects: Amateurs and professionals in Berkeley Museum of Vertebrate Zoology. Social Studies of Science 19: 387-420.

Staszewski, Wojciech (2009). Siła spokoju [The power of peace]. Gazeta Wyborcza, December 30; http://www.archiwum.wyborcza.pl/Archiwum/1,0,7136829,20091216RP-DGW,Sila_spokoju,zwykly.html (accessed: 10.10.2010).

Stein, Jeannine (2010). Yoga may be better at reducing anxiety and lifting mood than other forms of exercise, study finds. Los Angeles Times, November 11.

Stinissen, Wilfrid (2000). Ani joga, ani zen: chrześcijańska medytacja głębi [Deep Calls to Deep: A Study in Christian Depth-Meditation]. Transl. by J. Iwaszkiewicz. Poznań: W drodze.

Strauss, Anselm (1978). A social world perspective. Studies in Symbolic Interaction 1: 119-128.

Strauss, Anselm (1982). Social world and legitimation processes. Studies in Symbolic Interaction 4: 171-190.

Strauss Anselm (1997). Mirrors and Masks. The Search for Identity. New Brunswick: Transaction Publishers.

Streeter, Chris C., Theodore H. Whitfield, Liz Owen, Tasha Rein, Surya K. Karri, Aleksandra Yakhkind, Ruth Perlmutter, Andrew Prescot, Perry F. Renshaw, Domenic A. Ciraulo and J. Eric Jensen (2010). Effects of yoga versus walking on mood, anxiety, and brain GABA levels: A randomized controlled MRS study. The Journal of Alternative and Complementary Medicine 16 (11): 1145-1152.

Sztompka, Piotr (2005). Socjologia wizualna: fotografia jako metoda badawcza [Visual sociology: photography as a research method]. Warszawa: Wydawnictwo Naukowe PWN.

Szyszko-Bohusz, Andrzej (1978). Joga - indyjski system filozoficzny, leczniczy i pedagogiczny [Yoga - The Indian Philosophical, Therapeutic, and Pedagogical System]. Wrocław: Zakład Narodowy im. Ossolińskich.

Teasdill, Wendy (2001). Joga na czas ciąży [Yoga for Pregnency]. Transl. by A. Bezpiańska-Oglęcka. Warsaw: Wydawnictwo "Bis." 
Vivekananda, Swami (1982). Karma-Yoga and Bhakti-Yoga. New York: Ramakrishna-Vivekananda Center of New York.

Vivekananda, Swami (2003). Raja Yoga. Leeds: Celephaïs Press.

Waskul, Dennis and Phillip Vannini (2006). Body/Embodiment. Symbolic Interaction and the Sociology of the Body. Hampshire: Ashgate.

Wciórka, Bogna and Jacek Wciórka (2008). Polacy o niepokojach, zagrożeniach i oczekiwaniach dotyczacych zdrowia psychicznego [Polish People on Concerns, Threads, and Expectation in Regard to Mental Health]; http://www.cbos.pl/SPISKOM. POL/2008/K 105_08.PDF (accessed: 26.07.2014).

Weber, Max (1984). Szkice z socjologii religii [Sociology of Religion]. Transl. by J. Prokopiuk, H. Wandowski. Warsaw: Książka i Wiedza.

Weintraub, Amy (2005). Joga na depresję [Yoga for Depression]. Transl. M. Stopa. Warsaw: Klub dla Ciebie.

Wilson, Bryan R. (1992). The Social Dimension of Sectarianism: Sects and New Religious Movements in Contemporary Society. Oxford: Oxford University Press.

Wyka, Anna (1993). Badacz społeczny wobec doświadczenia [The Researcher vis-à-vis Research Experience]. Warsaw: Wydawnictwo IFiS PAN.

Ziółkowski, Łukasz (2010). Jogin w Kościele [Yogi in the Church]. Tygodnik Powszechny 22 (3177); http://tygodnik.onet.pl/wiara/jogin-w-kosciele/6j53c (accessed: 12.06.2010).

Zotz, Volker (2007). Historia filozofii buddyjskiej [The History of Buddhist Philosophy]. Transl. by M. Nowakowska. Kraków: Wydawnictwo WAM. 
REVIEWER

Grażyna Woroniecka

INITIATING EDITOR

Monika Borowczyk

\section{EDITOR OF ŁÓDŹ UNIVERSITY PRESS \\ Katarzyna Gorzkowska}

\section{TYPESETTING}

Munda - Maciej Torz

COVER DESIGN

Łukasz Orzechowski

COVER IMAGE

(C) Depositphotos.com/dimaberkut

First Edition. W.06863.15.0.M

Publisher's sheets 14.5; printing sheets 14.125 